\title{
Minimizing the discrimination time for quantum states of an artificial atom
}

\author{
Zur Erlangung des akademischen Grades eines \\ DOKTORS DER NATURWISSENSCHAFTEN (Dr. rer. nat.) \\ von der KIT-Fakultät für Physik des \\ Karlsruher Instituts für Technologie (KIT) \\ angenommene \\ Dissertation \\ von
}

M.Sc. Ivan Takmakov

Tag der mündlichen Prüfung:

Hauptreferent:

Korreferentin:

Betreuer:
28.01.2022

Prof. Dr. Wolfgang Wernsdorfer

Dr. Martina Esposito

Dr. Ioan M. Pop 



\section{Contents}

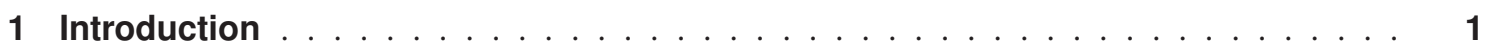

2 Circuit Quantum Electrodynamics . . . . . . . . . . . . . . . . . . . 3

2.1 Quantum electric circuits . . . . . . . . . . . . . . . . . 3

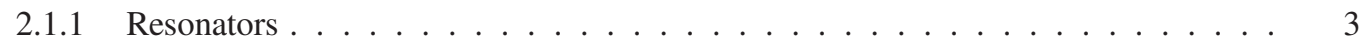

2.1 .2 Josephson junction $\ldots \ldots \ldots \ldots \ldots \ldots \ldots$

2.1 .3 Superconducting qubits $\ldots \ldots \ldots \ldots \ldots \ldots$

2.2 Readout and decoherence . . . . . . . . . . . . . . . . . . 7

2.2 .1 Open quantum system . . . . . . . . . . . . . . . . 7

2.2 .2 Input-output theory $\ldots \ldots \ldots \ldots \ldots$

2.2 .3 Dispersive readout . . . . . . . . . . . . . . . . . . . . . . . 12

2.3 Josephson parametric amplifier . . . . . . . . . . . . . . . . . . . . . 17

2.3.1 Principles of parametric amplification . . . . . . . . . . . . . . 17

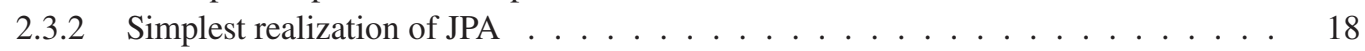

2.3.3 Important characteristics of JPAs . . . . . . . . . . . . . 22

3 Dimer Josephson Junction Array Amplifier (DJJAA) $\ldots \ldots \ldots \ldots$

3.1 Theoretical model . . . . . . . . . . . . . . . . . . . . . . 27

3.1 .1 Essential requirements for non-degenerate gain . . . . . . . . . . . . . . 30

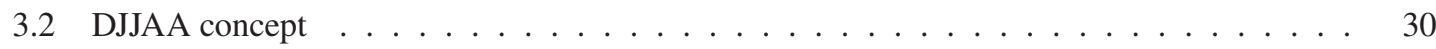

3.2 .1 Josephson junction array $(\mathrm{JJA}) \quad \ldots \ldots \ldots \ldots$

3.2 .2 Dimerization of a JJA . . . . . . . . . . . . . . . . . . . 33

3.3 Experimental realization . . . . . . . . . . . . . . . . . . . . . . . 34

3.4 Power calibration . . . . . . . . . . . . . . . . . . . . . 37

3.4.1 Scattering from a transmon qubit coupled to a transmission line . . . . . . . . 37

3.4.2 Scattering from a qubit-resonator system . . . . . . . . . . . . . . 39

4 High-power quantum jumps detection $\ldots \ldots \ldots \ldots \ldots \ldots \ldots$

4.1 Measurement strength . . . . . . . . . . . . . . . . . . . . 41

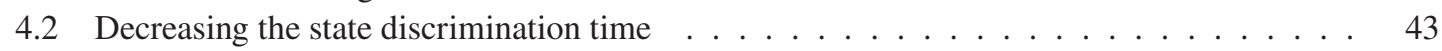

4.3 Speed-up and detection . . . . . . . . . . . . . . . . . . . . 44

4.4 Setup and qubit characterization . . . . . . . . . . . . . . . . . . 46

4.5 Bayesian detection applied . . . . . . . . . . . . . . . . . . . . . 49

4.6 Steady-state squeezing . . . . . . . . . . . . . . . . . . . 51

5 Towards parametric amplifier resilient to magnetic fields up to few Tesla . . . . . . 55

5.1 Granular Aluminum $(\mathrm{grAl}) \quad \ldots \ldots \ldots \ldots \ldots$. . . . . . . . . . . . . . . . . . . . . . 55

5.2 Nonlinearity of a grAl strip $\ldots \ldots \ldots \ldots \ldots \ldots$

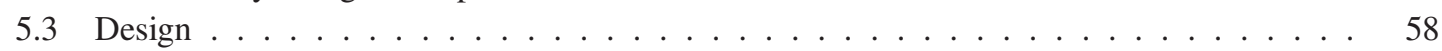

5.4 Experimental results . . . . . . . . . . . . . . . . . . . . . . . . . . 60

5.4 .1 Magnetic field resilience . . . . . . . . . . . . . . . . . . . 62

5.4 .2 Operating GrADPA in magnetic field . . . . . . . . . . . . 63 




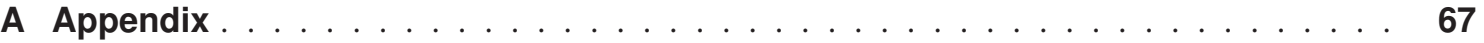

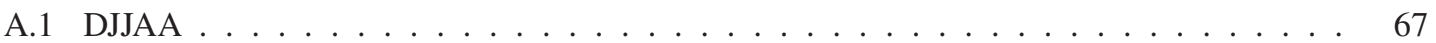

A.1.1 Capacitance and inductance matrices . . . . . . . . . . . . . . . 67

A.1.2 E-beam-lithography DJJAA . . . . . . . . . . . . . . . . . . . 68

A.1.3 Elastic scattering on a two-level system . . . . . . . . . . . . . . . . . . 69

A.2 High-power quantum jumps detection . . . . . . . . . . . . . . . . . . 71

A.2.1 Fluxonium parameters . . . . . . . . . . . . . . . . . . . . 71

A.2.2 Example of gain obtained on the fluxonium readout resonator . . . . . . . . 71

A.2.3 Measurement setup . . . . . . . . . . . . . . . . . . . . . . 72

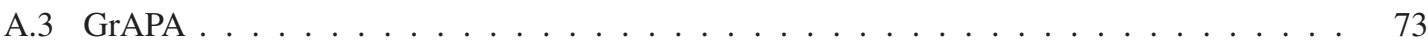

A.3.1 Compensation of the perpendicular field . . . . . . . . . . . . 73

A.3.2 High-power issues at the interface between materials with different kinetic inductance 74

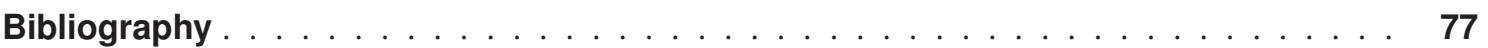

List of Publications $\ldots \ldots \ldots \ldots \ldots \ldots \ldots \ldots$

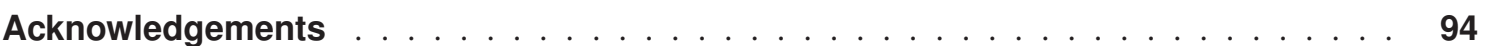




\section{Introduction}

During the 20th century, advances in quantum mechanics led to a number of inventions, such as lasers and semiconductor transistors, which have been making a huge impact on both modern technological progress and daily life. These inventions were possible due to the newly acquired understanding of quantum phenomena defining the behavior of macroscopic objects, e.g. energy spectrum of metals and semiconductors. Nowadays, scientific progress has enabled control over individual quantum systems, such as trapped ions [BKRB08; BCMS19; $\mathrm{MCD}^{+}$21], nitrogen vacancies in diamonds [ $\mathrm{DCJ}^{+}$07; Neb20], individual electron spins [LD98; Kan98; $\mathrm{CSDF}^{+}$21], molecular spins [BW08; $\mathrm{SKD}^{+}$16; MPW21], and superconducting qubits [NPT99;

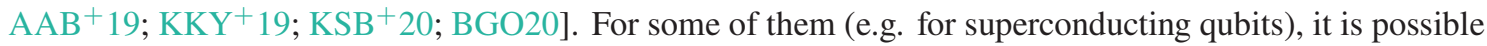
to not only utilize nature-given objects, but rather to engineer an artificially-made quantum system with desired energies and properties.

This allows for the development of (truly) quantum technologies relying on the very basic principles of quantum mechanics, such as entanglement and superposition. Currently, different applications of these technologies can be summarized as quantum information processing, including quantum cryptography, quantum sensing, and quantum computation. In quantum cryptography [Kim08; $\left.\mathrm{XMZ}^{+} 20\right]$, entangled quantum objects are used to realize key distribution which is protected by fundamental physical laws. Non-classical quantum states are utilized in quantum sensing to overcome the Heisenberg uncertainty principle and significantly increase measurement speed and precision, which is essential for axion search $\left[\mathrm{MPB}^{+}\right.$19; Leh21] and gravitational wave detection [AAAe16]. Fundamentally different computational algorithms can be run on quantum computers [Fey; DiV95], profiting from superposition and entanglement of states of multiple two-level systems, denoted quantum bits or qubits.

Superconducting quantum circuits are a prominent platform for quantum computation and sensing. The information is stored in quantum degrees of freedom of Cooper pair condensate in nano- and microfabricated resonators made of superconducting elements (both linear and nonlinear). Typically operating in 1-10 GHz frequency range, these resonators can be coupled between each other and readout lines with nearly arbitrary coupling strengths, enabling ultrastrong coupling regime [YFA $\left.{ }^{+} 17\right]$. The utilization of low-loss nonlinear elements such as Josephson junctions [Jos62] allows to engineer the energy spectrum of quantum systems and create artificial atoms. Recently, quantum supremacy was claimed to be achieved on a programmable superconducting quantum processor $\left[\mathrm{AAB}^{+} 19\right]$. The macroscopic nature, while providing versatility, comes with a price: superconducting circuits are coupled to numerous mesoscopic systems $\left[\mathrm{SDH}^{+} 19 ; \mathrm{GMF}^{+} 17 ; \mathrm{BVB}^{+} 21\right.$; MCL19] limiting lifetimes of superconducting qubits. Despite several decades of development of superconducting qubits, mitigating these unwanted interactions is still an ongoing research of major importance, and different fabrication [SBW $\left.{ }^{+} 20 ; \mathrm{OSB}^{+} 21\right]$, material [KTY $21 ; \mathrm{PRM}^{+}$21], and design [BKP13; $\left.\mathrm{GMDP}^{+} 21\right]$ improvements are being investigated.

Similar to any other platform, for superconducting-circuit-based quantum technologies it is instrumental to be able to readout a state of a superconducting artificial atom. The readout relies on the ability to infer the state of the atom in a time significantly shorter than its relaxation time. This capability is crucial for the implementation of quantum error correction algorithms, mandatory for quantum computation $\left[\mathrm{RDN}^{+} 12 ; \mathrm{RDW}^{+} 12 ; \mathrm{SPL}^{+} 14 ; \mathrm{KBF}^{+} 15 ; \mathrm{OPH}^{+} 16\right]$. Additionally, continuous measurement of quantum 
jumps between discrete levels of a superconducting artificial atom [VSS11] can be used as a detection tool, informing on the interactions between the quantum system and the environment, such as quasiparticles $\left[\mathrm{RBT}^{+} 13 ; \mathrm{VPS}^{+} 14 ; \mathrm{SDH}^{+}\right.$19]. For all these applications it is important to be able to discriminate the state of the quantum system as fast as possible.

The speed of high-fidelity single-shot readout improved over the last decade [ $\left.\mathrm{JSM}^{+} 14 ; \mathrm{WKG}^{+} 17\right]$, mainly thanks to the development of near-quantum limited Josephson parametric amplifiers [CBL07; YIW ${ }^{+} 08$; $\mathrm{MWB}^{+}$14; $\mathrm{RFN}^{+}$12; EW14; $\mathrm{MOH}^{+} 15$; RD16] and the utilization of Purcell filters [BLH ${ }^{+}$15]. An additional tuning knob allowing to speed-up the measurement is the readout drive power. However, as the readout power is increased, superconducting qubits typically suffer from increased transition rates and leakage outside of the computational space. In practice $\left[\mathrm{JMS}^{+} 12 ; \mathrm{WKG}^{+} 17 ; \mathrm{MMS}^{+} 19\right]$, these effects bound the photon number for quantum non-demolition (QND) readout, typically to $\bar{n}<10$, for reasons which are currently the subject of theoretical modelling [BGB09; MPT20; PMT20].

In the scope of this thesis, by using the remarkable insensitivity to $\bar{n}$ of the ground and excited state lifetimes of a granular Aluminum (grAl) fluxonium [GSG $\left.{ }^{+} 19\right]$ artificial atom, recently reported in [GSG $\left.{ }^{+} 21\right]$, the enhancement of the QND detection speed of quantum jumps with increasing $\bar{n}$, up to $\bar{n}=110$, is demonstrated. By utilizing a Bayesian filtering method [SPL ${ }^{+}$14; Kor16; WQL14; WMKS ${ }^{+}$16; FLQL16; $\mathrm{RMA}^{+} 10 ; \mathrm{SCID}^{+}$16], it was possible to achieve detection times up to 3 times faster than the time required for transitions between the readout resonator steady states.

To handle the large $\bar{n}$, a high dynamic range dimer Josephson junction array parametric amplifier [WTR +20 ] was developed. The amplifier, referred to as DJJAA, consists of a dispersion-engineered Josephson junction array, and can be operated in a non-degenerate regime [RD16], protecting qubit from pump leakage effects by ensuring frequency detuning between pump and signal tones of few hundreds of MHz. Fabricated with a standard two-step optical lithography, this amplifier is low-cost and accessible to a wide community.

Some areas of research, such as hybrid systems $\left[\mathrm{BPK}^{+} 16 ; \mathrm{CSDF}^{+} 21 ; \mathrm{MPW} 21\right]$ or search of axions [IR18; $\mathrm{OSF}^{+}$19], could profit from quantum-limited parametric amplifiers. However, since magnetic fields in the Tesla range are often required, Josephson-junction-based amplifiers cannot be properly utilized because the junction's critical current is suppressed $\left[\mathrm{SWP}^{+} 19\right]$. To enable high-magnetic-field quantum detection, the first generation of granular aluminum parametric amplifier (GrAPA) was developed in the scope of this thesis. Owing to nonlinearity of granular aluminum (grAl) $\left[\mathrm{MGK}^{+} 18\right]$ and resilience of grAl to in-plane fields up to few Tesla [BRW ${ }^{+}$20], the amplifier demonstrated $20 \mathrm{~dB}$ of gain in magnetic fields up to $1 \mathrm{~T}$. While presented experiments revealed a number of required optimizations, this work opens a way to utilize the current progress in the development of Josephson parametric amplifiers in close vicinity of hybrid quantum devices, where magnetic field shielding is not possible or practical. Additionally, utilization of GrAPAs can enhance measurement efficiency of the currently ongoing search for axions [IR18; OSF $\left.{ }^{+} 19\right]$ since the amplifier can be placed close to (or directly integrated into [EKT $\left.{ }^{+} 19\right]$ ) the readout resonator thus minimizing cable losses.

This thesis is organized as follows: first, an introduction to circuit quantum electrodynamics is given in chapter 2, focusing on subjects which are instrumental for readout. Chapter 3 reports on development of a dimer Josephson junction array amplifier $\left[\mathrm{WTR}^{+} 20\right]$ and utilization of scattering of microwave radiation on superconducting qubits for power calibration. Chapter 4 is dedicated to minimizing quantum jumps detection time $\left[\mathrm{TWF}^{+} 21\right]$ made possible by the unique combination of a dimer Josephson junction array amplifier with a large dynamic range, and the fact that the readout of a granular aluminum fluxonium artificial atom remained quantum-non-demolition at relatively large photon numbers in the readout resonator. In chapter 5, a granular aluminum parametric amplifier resilient to magnetic fields up to $1 \mathrm{~T}$ is discussed. 


\section{Circuit Quantum Electrodynamics}

This chapter intends to give an introduction to circuit Quantum Electrodynamics (cQED). It explains the concepts of both linear and non-linear quantum circuits and how the state of a quantum system can be measured, as well as the operating principle and important characteristics of Josephson parametric amplifiers.

Circuit quantum electrodynamics is a field that is more than 20 years old, and there is a number of excellent reviews [BGO20; $\left.\mathrm{KKY}^{+} 19\right]$ providing much more detailed information than this chapter does. For details about qubits and quantum computation, the interested reader is referred to $\left[\mathrm{KSB}^{+} 20\right]$. Nice introductions to the readout can be found in $\left[\mathrm{CDG}^{+}\right]$. For more information about Josephson parametric amplifiers, one can use [RD16; Aum20]

\subsection{Quantum electric circuits}

Superconducting quantum circuits have been proven to be easy to couple and manipulate while having lifetimes sufficient for quantum information processing. Here, the basic circuit building blocks and requirements are discussed.

\subsubsection{Resonators}

The simplest yet important quantum electric circuit is a lossless parallel LC resonator. Its classical Hamiltonian can be written as

$$
H=\frac{\Phi^{2}}{2 L}+\frac{Q^{2}}{2 C}
$$

where the node flux $\Phi$ is an integral of the voltage drop on the capacitor,

$$
\Phi(t)=\int_{\infty}^{t} V\left(t^{\prime}\right) d t^{\prime}=L I(t)
$$

The coordinate and momentum of this Hamiltonian are charge and flux, since $\frac{\partial H}{\partial Q}=\dot{\Phi}$ and $\frac{\partial H}{\partial \Phi}=-\dot{Q}$. Following the standard quantization procedure [VD17], they can be converted to quantum-mechanical operators $\hat{\Phi}$ and $\hat{Q}$ obeying the commutation relation $[\hat{\Phi}, \hat{Q}]=i \hbar$. The Hamiltonian can be then rewritten in terms of annihilation and creation operators:

$$
\hat{H}=\hbar \omega\left(a^{+} a+1 / 2\right)
$$

where the annihilation operator is given by

$$
a=\frac{\hat{\Phi}+i \hat{Q}}{\sqrt{2 \hbar Z_{\mathrm{res}}}}
$$


with $Z_{\text {res }}=\sqrt{L / C}$ being the resonator impedance. To operate a resonator in the quantum regime, two conditions must be fulfilled. First, the oscillator has to be decoupled from uncontrollable degrees of freedom $\left[\mathrm{MWG}^{+} 20\right]$ (e.g. radiation and dielectric losses), such that the gap between energy levels is much bigger than their linewidth. For superconducting resonators, the routinely achievable intrinsic quality factors are $Q_{\mathrm{i}} \sim 10^{3}-10^{7}$, which fulfills the low-loss requirement. Secondly, energy of thermal excitations has to be $k T \ll \hbar \omega$. Since temperature corresponding to $1 \mathrm{GHz}$ is $T_{1 \mathrm{GHz}} \approx 50 \mathrm{mK}$ and typical resonator frequencies are in the 4-12 GHz range, this condition is satisfied for a typical dilution refrigerator operating at $20 \mathrm{mK}$.

\subsubsection{Josephson junction}

Energy levels of an oscillator are equidistant, and the resonator mean energy is given by the number of circulating photons. To form a qubit, one needs to be able to individually address the transition of interest (e.g. $|0\rangle \rightarrow|1\rangle$ ). To achieve that, a low-loss nonlinear element needs to be introduced into the circuit, thus changing energy levels inhomogeneously.

The standard nonlinear element of choice in superconducting electronics is the Josephson junction [Tin04; Sch97]. It was first predicted by Josephson [Jos62] that supercurrent can flow between two superconductors separated by a weak link. While the exact nature of the weak link can vary (constriction in a wire, normal metal layer, ferromagnetic layer, etc.), in this work I utilize superconductor-insulator-superconductor junctions with a weak link formed of a thin insulating layer. This system can be described by two Josephson equations,

$$
\begin{array}{r}
I=I_{\mathrm{c}} \sin \left(\frac{2 \pi \Phi}{\Phi_{0}}\right) \\
\dot{\Phi}=V,
\end{array}
$$

where $I$ is the current flowing through the junction, $I_{\mathrm{c}}$ is the junction critical current, $V$ is the voltage across the junction, $\Phi_{0}$ is the flux quantum, and $\varphi=2 \pi \Phi / \Phi_{0}$ is the difference of condensate phases between two superconductors. If $I$ exceeds $I_{\mathrm{c}}$, Cooper pairs are broken, and the Josephson junction becomes resistive. For quantum information processing, it is thus crucial to keep operating currents well below $I_{\mathrm{c}}$. From Eqs. (2.5),(2.6), for ac supercurrents below critical in the absence of charging effects, voltage and current are linked as

$$
V=\frac{\Phi_{0}}{2 \pi I_{\mathrm{c}}} \frac{\dot{I}}{\sqrt{1-\left(I / I_{\mathrm{c}}\right)^{2}}},
$$

meaning that Josephson junction can be thought of as a nonlinear inductor

$$
L_{\mathrm{J}}(I)=\frac{\Phi_{0}}{2 \pi I_{\mathrm{c}}} \frac{1}{\left.\sqrt{1-\left(I / I_{\mathrm{c}}\right.}\right)^{2}}=\frac{L_{\mathrm{J}}}{\left.\sqrt{1-\left(I / I_{\mathrm{c}}\right.}\right)^{2}} .
$$

The inductive energy associated with Cooper pair tunneling is

$$
E=E_{\mathrm{J}}\left(1-\cos \frac{2 \pi}{\Phi_{0}} \Phi\right)
$$

where $E_{\mathrm{J}}=\frac{\Phi_{0} I_{\mathrm{c}}}{2 \pi}$ is referred to a Josephson energy. In addition to the tunnel energy, there is also a charging energy $Q^{2} / 2 C_{\mathrm{J}}$ due to finite capacitance $C_{\mathrm{J}}$ between superconducting islands of the junction. The total Hamiltonian is given by

$$
H=\frac{Q^{2}}{2 C_{\mathrm{J}}}+E_{\mathrm{J}}\left(1-\cos \frac{2 \pi}{\Phi_{0}} \Phi\right) .
$$


In contrast to Eq. (2.1), this Hamiltonian is nonlinear due to the cosine term replacing the magnetic energy $\Phi^{2} / 2 L$, which gives an anharmonic spectrum. Provided that the linewidth of energy levels is much smaller than the distance between them, this system can be thought of as an artificial atom.

\subsubsection{Superconducting qubits}

While the Josephson junction provides the required nonlinearity to build a qubit, there is a number of important characteristics defining whether this qubit is "good", such as susceptibility to different noise sources, coupling strength to control lines, or anharmonicity $\alpha=E_{12}-E_{01}$. To be able engineer these parameters and optimize qubit performance, the Josephson junction (or junctions) is typically inserted into some electric circuit.

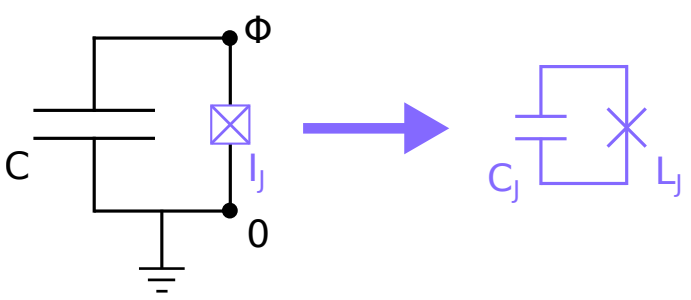

Figure 2.1: Schematic of a superconducting artificial atom: a Josephson junction shunted with a capacitor (left image). Josephson junction schematic representation used here represents an ideal Josephson nonlinear inductance (Eq. (2.7)) shunted with a junction capacitance $C_{\mathrm{J}}$ between the electrodes. It is thus applicable only for currents below $I_{\mathrm{J}}$.

One of the simplest examples of qubit engineering is a transmon qubit $\left[\mathrm{KYG}^{+} 07\right]$ schematically shown in Fig. 2.1. It consists of a Josephson junction shunted with a capacitance $C \gg C_{J}$. It's Hamiltonian is similar to Eq. (2.9), and is equal to

$$
H=\frac{\left(Q-Q_{g}\right)^{2}}{2\left(C+C_{\mathrm{J}}\right)}+E_{\mathrm{J}}\left(1-\cos \frac{2 \pi}{\Phi_{0}} \Phi\right)=4 E_{\mathrm{C}}\left(N-N_{g}\right)^{2}+E_{\mathrm{J}}(1-\cos \varphi),
$$

where $\mathrm{N}$ is the number of tunneled Cooper pairs in the units of $2 e, N_{g}$ is the offset charge representing the external field bias, and $E_{\mathrm{C}}=e^{2} / 2\left(C+C_{\mathrm{J}}\right)$ is the charging energy of the qubit. The Hamiltonian's spectrum is controlled by the ratio $E_{\mathrm{J}} / E_{\mathrm{C}}$. When either $E_{\mathrm{J}} / E_{\mathrm{C}} \ll 1$ or $E_{\mathrm{J}} / E_{\mathrm{C}} \gg 1$, eigenstates of the Hamiltonian are given by the eigenstates of charge or flux operator, respectively, due to the corresponding energy being dominant. If the charging energy dominates, the system is in a charge qubit regime [NPT99], and the offset charge has a strong influence on the transition frequencies. Consequently, the unavoidable charge noise creates fluctuations of the artificial atom transition frequencies and leads to dephasing. This problem can be addressed by designing the circuit in the so-called transmon regime by using such shunting capacitances that $E_{\mathrm{J}} / E_{\mathrm{C}}=20-80$. In this case, variation of energy with the offset charge is suppressed by $\exp \left(-\sqrt{8 E_{\mathrm{J}} / E_{\mathrm{C}}}\right)$. This comes with a price of anharmonicity reduction. However, the anharmonicity scaling is approximately linear, which makes the trade-off beneficial for $E_{\mathrm{J}} / E_{\mathrm{C}} \approx 50$.

The full description and derivations for transmon qubit can be fond in $\left[\mathrm{KYG}^{+} 07\right]$. Here, I focus on approximations sufficient for the scope of the thesis. Thanks to phase variations being small and offset charge having negligible influence of the eigenenergies, the Hamiltonian can be truncated to the first nonlinear term of the cosine expansion

$$
H \approx 4 E_{\mathrm{C}} n^{2}+\frac{1}{2} E_{\mathrm{J}} \varphi^{2}-\frac{1}{4 !} E_{\mathrm{J}} \varphi^{4} \approx\left(\sqrt{8 E_{\mathrm{J}} E_{\mathrm{C}}}-E_{\mathrm{C}}\right) a^{+} a-\frac{E_{\mathrm{C}}}{2} a^{+} a^{+} a a,
$$


where, similar to Eq. (2.4),

$$
\begin{gathered}
\varphi=\left(\frac{8 E_{\mathrm{C}}}{E_{\mathrm{J}}}\right)^{1 / 4}\left(a^{+}+a\right) \\
n=i\left(\frac{E_{\mathrm{J}}}{8 E_{\mathrm{C}}}\right)^{1 / 4}\left(a^{+}-a\right) .
\end{gathered}
$$

This Hamiltonian is commonly known as a Kerr Hamiltonian, with fundamental mode frequency $\omega_{0}=$ $\left(\sqrt{8 E_{\mathrm{J}} E_{\mathrm{C}}}-E_{\mathrm{C}}\right) / \hbar$ and Kerr nonlinearity $K=-E_{\mathrm{C}} / \hbar$ indicating by how much the resonance frequency shifts when one photon is added to resonator population, thus defining the anharmonicity $\alpha$. For transmons, typical $\alpha$ is in the $100 \mathrm{MHz}$ range, which is small compared to fundamental mode frequencies of few $\mathrm{GHz}$. Nevertheless, transmons can be used as artificial atoms thanks to achievable narrow linewidth of the modes.

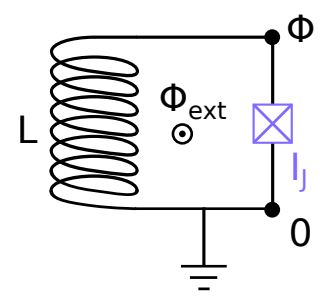

Figure 2.2: Schematic of a fluxonium artificial atom: the Josephson junction with critical current $I_{\mathrm{J}}$ and capacitance $C_{\mathrm{J}}$ shunted with the superinductor $L$. The external magnetic flux $\Phi_{\text {ext }}$ is used to tune the atom's energy levels.

Despite having simple structure and small anharmonicity, transmon qubits are currently one of the most used qubits in $\mathrm{CQED}$, allowing to perform quantum computations [MWHH21] and achieve quantum supremacy $\left[\mathrm{AAB}^{+} 19 ; \mathrm{ZCCe} 21\right]$. However, there are many other qubit types, utilizing different circuit topologies, number of Josephson junctions, and $E_{\mathrm{J}} / E_{\mathrm{C}}$ aiming to increase qubit anharmonicity and coherence times [MKGD09; $\mathrm{YGK}^{+}$16; $\mathrm{YSK}^{+}$20; $\mathrm{YPN}^{+}$21; $\mathrm{BKP}$ 13; $\mathrm{GMDP}^{+} 21$ ]. One of the most promising designs is the fluxonium artificial atom [MKGD09]. Fig. 2.2 shows its electrical schematic consisting of a Josephson junction shunted with a superinductor $L \gg L_{\mathrm{J}}$. The superinductor is typically made of an array of Josephson junctions [MKGD09; $\mathrm{PGC}^{+} 14$; $\mathrm{NLS}^{+}$19] or a kinetic inductance material [ $\mathrm{GSG}^{+}$19]. The fluxonium is described by the Hamiltonian

$$
H=\frac{Q^{2}}{2 C_{\mathrm{J}}}+\frac{\Phi_{L}^{2}}{2 L}+E_{\mathrm{J}} \cos \frac{2 \pi}{\Phi_{0}} \Phi,
$$

where $\Phi_{L}$ is the flux associated with the superinductance. It is linked with the Josephson-junction flux $\Phi$ and external flux $\Phi_{\text {ext }}$ via [Tin04]

$$
\Phi_{L}-\Phi+\Phi_{\mathrm{ext}}=m \Phi_{0}
$$

By including the external flux into the inductive term to make the Hamiltonian viable for time-dependent fields [YSK19], Eq. (2.14) becomes

$$
H=\frac{Q^{2}}{2 C_{\mathrm{J}}}+\frac{1}{2 L}\left(\Phi-\Phi_{\mathrm{ext}}+m \Phi_{0}\right)^{2}+E_{\mathrm{J}} \cos \frac{2 \pi}{\Phi_{0}} \Phi .
$$

To obtain the system's eigenmodes, this Hamiltonian is numerically diagonalized according to the procedure described in Ref. [SKV $\left.{ }^{+} 16\right]$. The typical anharmonicity of a fluxonium is in the GHz range, allowing to implement fast control gates and perform high-power readout without exciting higher energy states. On top of that, this design has a reduced sensitivity to charge noise [MKGD09]. Currently, the highest reported coherence times of fluxonium artificial atoms are in the millisecond range $\left[\mathrm{PGC}^{+} 14 ; \mathrm{LNG}^{+} 18 \mathrm{a}\right.$; NLS ${ }^{+} 19$; $\mathrm{SFM}^{+} 21$ ], which is the state-of-the-art record for superconducting qubits. 


\subsection{Readout and decoherence}

Quantum systems discussed so far were considered isolated from the environment. However, this is merely an idealized picture. In the experimental reality, they are coupled to numerous systems, both wanted and unwanted. In this section I introduce the so-called input-output formalism to treat systems coupled to the continuum, show how this coupling affects the systems, and argue that this can be used to measure the state of a quantum system.

\subsubsection{Open quantum system}

The most straightforward way to interact with a quantum system is to couple it to a continuum, thus realizing a so-called open quantum system. Unfortunately, this interaction not only creates a communication channel, but also opens a channel for decoherence. To demonstrate that, one can consider a toy model of a qubit coupled to one bath with a coupling strength $\kappa$. The evolution of the qubit's density matrix is described by a Lindblad-form master equation [Car02]

$$
\dot{\rho}=-\frac{i}{\hbar}[H, \rho]+\kappa\left(n_{\mathrm{TL}}+1\right) \hat{D}(\sigma) \rho+\kappa n_{\mathrm{TL}} \hat{D}\left(\sigma^{+}\right) \rho
$$

where $n_{\mathrm{TL}}=n_{\mathrm{TL}}\left(\omega_{\mathrm{q}}\right)$ is the number of thermal photons in the transmission line at the qubit frequency, following the Bose-Einstein statistics, and the operator $\hat{D}(X) Y=X Y X^{+}-\frac{1}{2}\left\{X^{+} X, Y\right\}$ represents the dissipation term. This equation describes dynamic of a two-level system with energy relaxation time $T_{1}=1 / \kappa$ and dephasing time $T_{2}=2 T_{1}$. In case of coupling to several baths with different couplings $\kappa_{i}$, total qubit decay rates are equal to sum of decay rates of different channels $T_{1}^{-1}=\sum_{i}^{n} \kappa_{i}$.

Whenever a qubit is coupled to a bath, the qubit's state lifetimes get reduced. On the other hand, signals sent through the transmission line (TL) get scattered on a quantum system, giving rise to such effects as elastic and inelastic nonlinear scattering $\left[\mathrm{AZA}^{+} 10 ; \mathrm{HWJ}^{+} 11\right]$. These effects are drive-power-dependent and can both be used for in situ power calibration $\left[\mathrm{HDSdG}^{+}\right.$20], which is instrumental for different measurement routines in cQED (e.g. determining Kerr coefficients or extracting quantum efficiency of the measurement setup). Remarkably, calibration accuracy for these methods does not depend on exact parameters and decay times of a qubit, as long as it is Purcell-limited [PTP46].

\section{Elastic scattering}
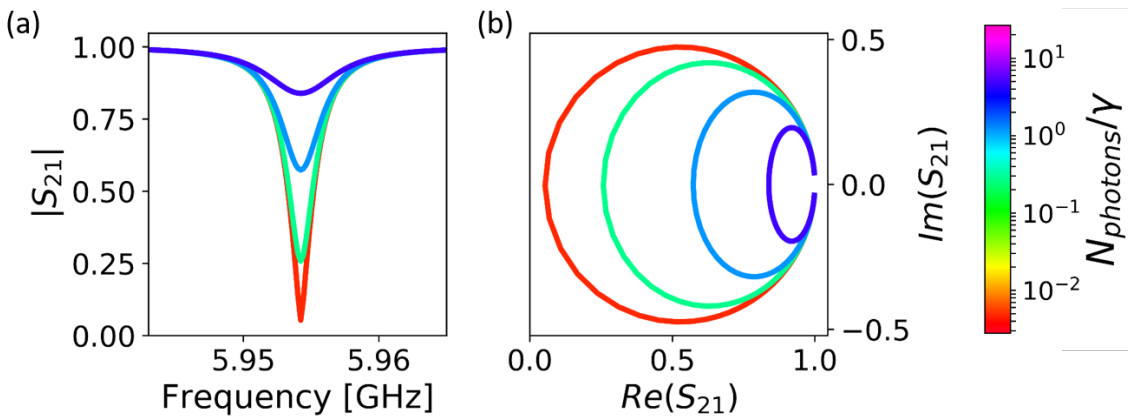

Figure 2.3: (a) Transmission as a function of frequency for different drive powers. Power corresponding to each line correspond to the color bar on the right-hand side. (b) Complex plane response parametric plot versus frequency. 
In case of elastic scattering, when a coherent continuous drive is sent in a vicinity of a qubit frequency, a fraction of the sent photons get absorbed, and the absorption coefficient is power-dependent. The transmission coefficient of that signal for the Purcell-limited case is equal to (see Appendix A.1.3):

$$
S_{21}=\frac{\kappa}{2 \gamma} \frac{1+i \delta \omega / \gamma}{1+(\delta \omega / \gamma)^{2}+\Omega^{2} / \kappa \gamma}
$$

Here $\delta \omega=\omega-\omega_{\mathrm{q}}$ is a detuning of the drive frequency from the qubit frequency, $\Omega=\frac{2 \kappa P}{\hbar \omega_{\mathrm{q}}}$ is the Rabi frequency which depends on the drive power $P$, and $\gamma=\kappa / 2+\gamma_{\phi}$ is the $T_{2}$ relaxation rate with the term $\gamma_{\varphi}$ accounting for qubit dephasing caused by frequency fluctuations or coupling to unwanted baths. For the ideal case of a lossless two-level system $\left(\gamma_{\varphi}=0\right)$, when $\omega_{\text {drive }}=\omega_{\mathrm{q}}$, a weak drive $(\Omega \ll \kappa)$ gets fully absorbed and re-emitted with a $\pi$ phase shift, while absorption of a strong drive $(\Omega \gg \kappa)$ is negligible due to the fact that qubit can only interact with one photon at a time, as can be inferred from Fig. 2.3. Since all coefficients of Eq. 2.18 can be experimentally extracted by applying a circle fit procedure $\left[\mathrm{PSB}^{+} 15\right]$ to a low-power response, power dependence of the transmission coefficient can indeed be utilized for power calibration purposes.

\section{Inelastic scattering}

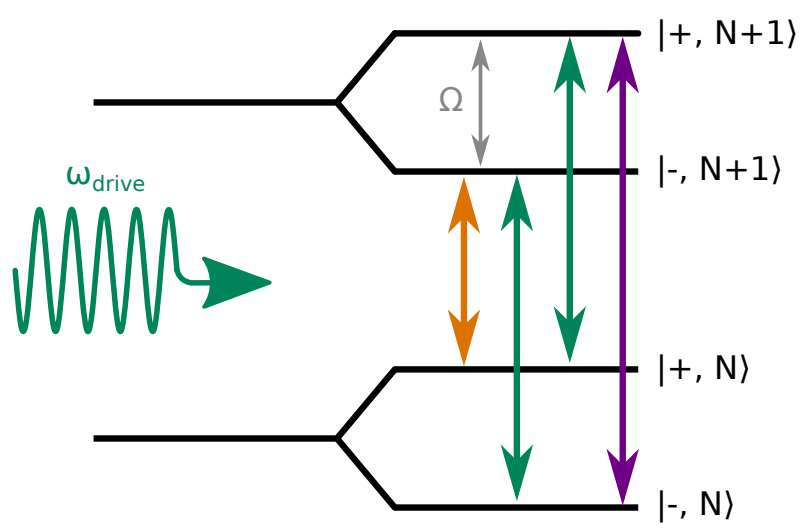

Figure 2.4: Sketch illustrating the Mollow triplet appearing due to hybridization of the qubit states in presence of a strong coherent drive. Green, orange, and magenta colors correspond to frequencies $\omega_{\text {drive }}, \omega_{\text {drive }}-\Omega$, and $\omega_{\text {drive }}+\Omega$ respectively.

When a two-level system interacts with a strong drive $(\Omega \gg \kappa)$, its states get dressed and levels are split by $\hbar \Omega$. This is known as Autler-Townes effect [AT55], and it is in fact a dynamical Stark shift. Assuming a strong drive, the Hamiltonian of the system can be written as

$$
H / \hbar=\omega_{\mathrm{q}} \sigma_{z}+\omega_{\text {drive }} a^{+} a+g\left(a^{+} \sigma+a \sigma^{+}\right)
$$

where $\sigma, \sigma^{+}$are the ladder Pauli operators. In the absence of coupling, the eigenenergies in the $(|0, N+1\rangle,|1, N\rangle)$ basis are:

$$
\begin{gathered}
E_{|0, N+1\rangle}=(N+1) \hbar \omega_{\text {drive }}-\hbar \omega_{\mathrm{q}} / 2 \\
E_{|1, N\rangle}=N \hbar \omega_{\text {drive }}+\hbar \omega_{\mathrm{q}} / 2
\end{gathered}
$$


If $\omega_{\text {drive }}=\omega_{\mathrm{q}}$, the off-diagonal interaction term $g\left(a^{+} \sigma+a \sigma^{+}\right)$splits levels, such that new eigenfrequeicies are equal to

$$
E_{| \pm, N\rangle}=(N-1 / 2) \hbar \omega_{\mathrm{q}} \pm \hbar g \sqrt{N}
$$

resulting in four energy levels. In the limit of large photon numbers $N \gg 1$, there are three unique transition frequencies between these levels $\omega_{\mathrm{q}}, \omega_{\mathrm{q}} \pm \Omega$, known as Mollow triplet [Mol69]. To observe this effect, one needs to apply a strong drive creating the hybridization, and probe the system with a weak tone. By fitting power dependence of the modes splitting, power calibration can be performed. Since measurement of frequency is generally more accurate than measurement of transmission coefficient, the Mollow triplet approach is a more precise power calibration method than the elastic scattering one.

\subsubsection{Input-output theory}

The master equation presented in the previous section only describes evolution of a quantum system while providing no information about radiated fields. For the readout, however, it is crucial to be able to link the measured radiated (or reflected) signal with the state of the system of interest. It can be done within the so-called input-output theory proposed by Gardiner and Collet [GC85]. Here I derive the main results and equations of this theory, focusing on a practical case of microwave transmission line coupled to a quantum system with defined creation and annihilation operators sketched in Fig. 2.5. A more detailed introduction to the input-output theory (IOT) can be fond in $\left[\mathrm{CDG}^{+}\right.$; $\left.\mathrm{BGO} 20\right]$.

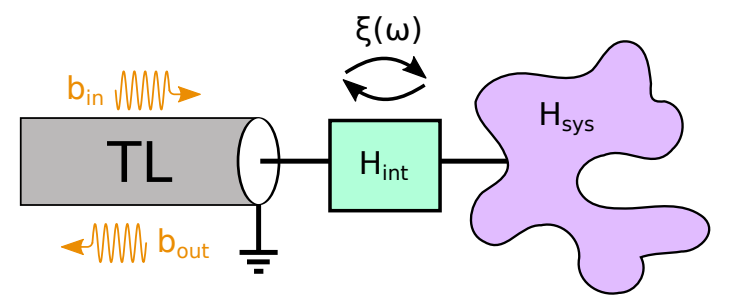

Figure 2.5: Sketch of the system under study: transmission line (TL) coupled to a system under study with Hamiltonian $H_{\text {sys }}$ with coupling strength $\xi(\omega)$. Here, $b_{\text {in }}$ and $b_{\text {out }}$ are the input and output fields, respectively.

We consider a quantum system described by Hamiltonian $H_{\text {sys }}$ coupled to a microwave transmission line (see Fig. 2.5). A semi-infinite transmission line can be modelled as a thermal bath $\left[\mathrm{CDG}^{+}\right]$with a Hamiltonian

$$
H_{\text {bath }}=\int_{-\infty}^{\infty} b^{+}(\omega) b(\omega) d \omega
$$

where the annihilation/creation operators satisfy $\left[b(\omega), b^{+}(\omega)\right]=\delta\left(\omega-\omega^{\prime}\right)$. A single bath model is used despite the fact that there are left and right propagating waves. It is possible due to the boundary condition at the right-hand side of the transmission line, which, in the absence of the probe system, yields zero current at this node. Because of the boundary condition, both left and right propagating waves can be described by the same operators, which simply implies the fact that the right propagating waves are at some point reflected at the open end and and become left propagating waves.

Interaction of the system and bath, assuming rotating wave approximation, is described by a Hamiltonian:

$$
\begin{gathered}
H=H_{\text {sys }}+H_{\text {bath }}+H_{\text {int }} \\
H_{\text {int }}=\hbar \int_{-\infty}^{\infty} d \omega \xi(\omega)\left[b^{+}(\omega) a+b(\omega) a^{+}\right] .
\end{gathered}
$$


While not specifying the exact system Hamiltonian, here we focus on evolution of the system's annihilation operator $a$. Heisenberg equations for $b(\omega)$ and $a$ are:

$$
\begin{array}{r}
\partial_{t} b(\omega)=-i w b(\omega)-i \xi(\omega) a \\
\partial_{t} a=-\frac{i}{\hbar}\left[a, H_{\mathrm{sys}}\right]+i \int_{-\infty}^{\infty} d \omega \xi(\omega) b(\omega) .
\end{array}
$$

Assuming $b(\omega)$ is known at some moment of time $t_{0}<t$ (which is the case if, for example, one sends a readout pulse), Eq. (2.26) can be integrated:

$$
b(\omega, t)=b_{0}(\omega) e^{-i w\left(t-t_{0}\right)}-i \int_{t_{0}}^{t} d t^{\prime} e^{-i \omega\left(t-t^{\prime}\right)} \xi(\omega) a\left(t^{\prime}\right) .
$$

First term of the right-hand part of this equation is a free evolution, while the second one describes an evolution due to the interaction with the quantum system. It thus makes sense to define the input field as

$$
b_{\text {in }}(t)=\frac{1}{\sqrt{2 \pi}} \int_{-\infty}^{\infty} d \omega e^{-i \omega\left(t-t_{0}\right)} b_{0}(\omega)
$$

where the normalization coefficient is chosen for the later convenience.

By substituting (2.26) into (2.27), one gets

$$
\partial_{t} a=-\frac{i}{\hbar}\left[a, H_{\mathrm{sys}}\right]+i \int_{-\infty}^{\infty} d \omega \xi(\omega)\left\{b_{0}(\omega) e^{-i \omega\left(t-t_{0}\right)}-i \int_{t_{0}}^{t} d t^{\prime} e^{-i \omega\left(t-t^{\prime}\right)} \xi(\omega) a\left(t^{\prime}\right)\right\} .
$$

To proceed further, it makes sense to simplify this expression by introducing the first Markov approximation $\xi(\omega)=\sqrt{\frac{\kappa}{2 \pi}}$. It can be done if the following conditions are fulfilled:

- Coupling strength has to be small compared to the system eigenfrequencies $\left(\kappa \ll \omega_{\text {sys }}\right)$. Although it is possible in $\mathrm{cQED}$ to achieve ultra strong coupling regime [YFA $\left.{ }^{+} 17\right]$, it is not the case for standard devices, and the typical ratio is $\kappa / \omega_{\text {sys }} \sim 10^{-1}-10^{-4}$.

- The system is Markovian, in the sense that there is no "memory" time and the evolution operator is linear. In $\mathrm{cQED}$, due to the standard operation temperatures of tens of $\mathrm{mK}$, the typical memory time is $\tau_{\text {memory }} \propto \hbar / k T \sim 0.1 \mathrm{~ns}$, which is negligible for normal readout and manipulation routines $\left[\mathrm{KKY}^{+} 19\right]$.

With that, and by using $\int_{-\infty}^{\infty} d \omega e^{-i \omega\left(t-t^{\prime}\right)}=2 \pi \delta\left(t-t^{\prime}\right)$ and $\int_{t_{0}}^{t} d t^{\prime} \delta\left(t-t^{\prime}\right) a\left(t^{\prime}\right)=\frac{1}{2} a(t)$, the last equation can be simplified to

$$
\partial_{t} a=-\frac{i}{\hbar}\left[a, H_{\mathrm{sys}}\right]-\frac{\kappa a(t)}{2}+\sqrt{\kappa} b_{\mathrm{in}}(t)
$$

This is one of the final equations, describing the evolution of the quantum system provided that the input field is known. A similar result can be obtained for the output field. In that case, the field is known at the moment $t_{1}>t$ :

$$
b_{\text {out }}(t)=\frac{1}{\sqrt{2 \pi}} \int_{-\infty}^{\infty} d \omega e^{-i \omega\left(t-t_{1}\right)} b_{1}(\omega) .
$$

Following the same routine, the Langevin equation can be obtained:

$$
\partial_{t} a=-\frac{i}{\hbar}\left[a, H_{\text {sys }}\right]+\frac{\kappa}{2} a(t)-\sqrt{\kappa} b_{\text {out }}(t) .
$$


It is important to mention here that since for the reflection measurement there is a boundary condition at the right end of the TL, the sign in front of $b_{\text {out }}$ is swapped.

Substituting (2.31) into (2.30) results in the standard input-output relation

$$
b_{\text {out }}(t)=-b_{\text {in }}(t)+\sqrt{\kappa} a(t),
$$

allowing to interpret the results of a quantum system readout.

\section{Resonator with intrinsic losses}

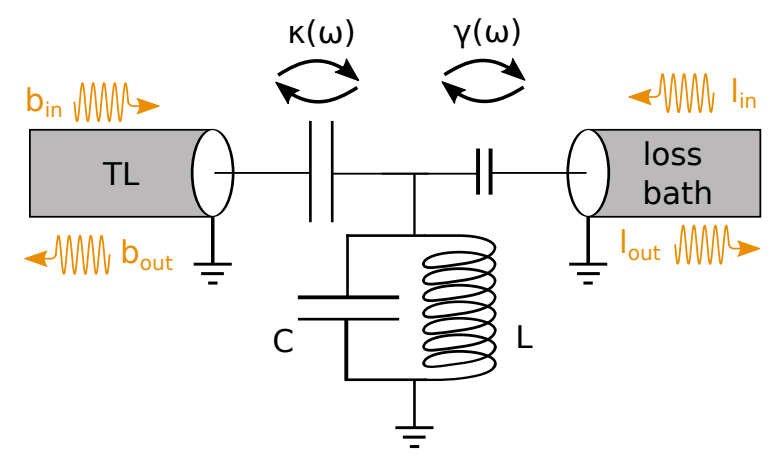

Figure 2.6: Sketch of a parallel LC resonator coupled to a readout transmission line (TL) with coupling strength $\kappa$. Intrinsic losses of the resonator are modelled with and additional transmission line with coupling strength $\gamma$.

To demonstrate the application of the IOT, lets consider the common practical case of a resonator with intrinsic losses coupled to a transmission line (see Fig. 2.7). To model intrinsic losses, an additional fictional transmission line is coupled to the resonator with a coupling strength $\gamma=\omega_{\text {res }} / Q_{i}$, where $Q_{i}$ is the internal quality factor. The Langevin equation for that system is

$$
\partial_{t} a=-\frac{i}{\hbar}\left[a, H_{\mathrm{sys}}\right]-\frac{\kappa+\gamma}{2} a(t)+\sqrt{\kappa} b_{\mathrm{in}}(t)+\sqrt{\gamma} l_{\text {in }}(t),
$$

which, in frequency space, transforms into

$$
a(\omega)=\frac{\sqrt{\kappa} b_{\text {in }}(\omega)}{i\left(\omega-\omega_{0}\right)+(\kappa+\gamma) / 2}+\frac{\sqrt{\gamma} l_{\text {in }}(\omega)}{i\left(\omega-\omega_{0}\right)+(\kappa+\gamma) / 2} .
$$

Experimentally, the resonator is characterised by measuring scattering parameters. In this case, the measurable scattering matrix has only one term - the reflection coefficient $S_{11}(\omega)=b_{\text {out }} / b_{\text {in }}$. By utilizing the IOT relation $b_{\text {out }}=-b_{\text {in }}+\sqrt{\kappa} a$, it can be written as

$$
S_{11}(\omega)=-1+\frac{\kappa}{i\left(\omega-\omega_{0}\right)+(\kappa+\gamma) / 2}
$$

In Fig. 2.7, the reflection coefficient is shown as a parametric plot versus frequency for different ratios of $\kappa$ and $\gamma$. Depending on the ratio, there are three resonator regimes:

- Overcoupled resonator $(\kappa \gg \gamma)$. The photons mainly leak out to the readout line, and only negligible part of information gets dissipated. The response is clearly visible in the phase of the reflected 
signal. This regime is instrumental for quantum information processing (e.g. for readout resonators $\left[\mathrm{WSB}^{+}\right.$04]).

- Critically coupled resonator $(\kappa=\gamma)$. The photon leak out rate is equal to the dissipation rate, and at resonance all input power is dissipated. This regime is optimal for measuring $Q_{i}$ and studying internal loss mechanisms $\left[\mathrm{SBO}^{+} 11 ; \mathrm{HVC}^{+} 19\right]$.

- Undercoupled resonator $(\kappa \ll \gamma)$. In this regime it is hard to measure the response, and in cQED undercoupled ports are mainly used to apply a drive or a pump tone without introducing significant losses.

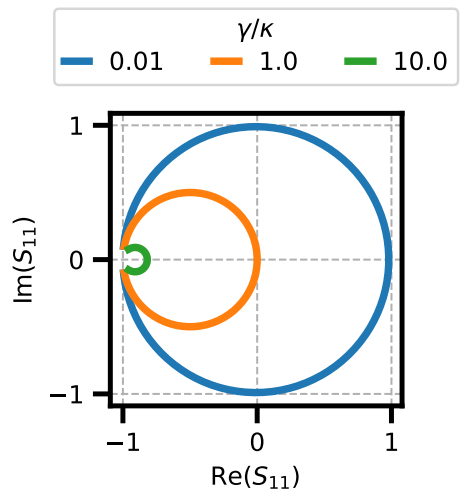

Figure 2.7: Reflection coefficient of resonator for different values of $\gamma$, corresponding to overcoupled (blue curve), critically coupled (orange curve), and undercoupled (green curve) resonator regimes.

\subsubsection{Dispersive readout}

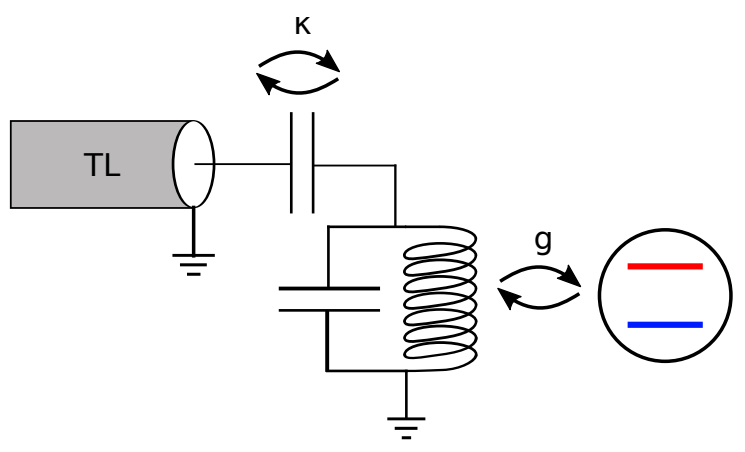

Figure 2.8: Scheme of a qubit-resonator system used for dispersive readout.

Generally, to perform a readout of a quantum system one needs to find a way to couple it to some quantum degree of freedom which can be measured by a classical apparatus. For superconducting qubits the typically used approach is a dispersive scheme adopted from cavity QED. A qubit is coupled to a readout resonator (either capacitively or inductively) with coupling strength $g$. As a result of this interaction, states of the qubit and the resonator hybridize, and frequency of the resonator thus becomes qubit-state-dependent. A readout tone reflected from the resonator acquires a qubit-state-dependent phase shift. Provided that the measurement signal-to-noise ratio (SNR) is sufficient, by measuring the output field one can infer the qubit state. 
Light-matter interaction in this case is described by the James-Cummings Hamiltonian

$$
H=\hbar \omega_{\mathrm{q}} \sigma_{z} / 2+\hbar \omega_{\mathrm{res}} a^{+} a+\hbar g\left(a^{+} \sigma+a \sigma^{+}\right),
$$

where we have used rotating wave approximation. The last term is responsible for single quanta interactions between the resonator photon population and qubit excitation. The "regime" of this coupled system depends on the ratio of coupling strength $g$ and frequency difference between the qubit and the resonator $\Delta=\omega_{\mathrm{q}}-\omega_{\text {res }}$. On resonance, with $|g / \Delta| \gg 1$, the bare qubit and resonator eigenstates become strongly hybridized and lose their individual character. As a result, energy is coherently swapped between two systems via so-called vacuum Rabi oscillations. This regime, although being valuable for quantum gates operations, is not desired in the context of quantum non-demolition measurement, for which the state of the measured system must not be changed by the act of measurement.

In the dispersive regime defined by $|g / \Delta| \ll 1$, qubit and resonator are weakly entangled. The Hamiltonian can be diagonalized perturbatively by using unitary transformation $U=\exp \left(\frac{g}{\Delta}\left(a^{+} \sigma-a \sigma^{+}\right)\right)$. By expanding $U^{+} H U$ to the first order of $\chi=2 g^{2} / \Delta$, one gets

$$
H_{\text {disp }}=U^{+} H U \approx \hbar \omega_{\mathrm{q}} \sigma_{z} / 2+\hbar \omega_{\text {res }} a^{+} a+\hbar \chi a^{+} a \sigma_{z} / 2 .
$$

This formula gives a good approximation as long as the resonator photon number fulfills $n \ll n_{\text {crit }}=$ $(\Delta / 2 g)^{2}$, assuming that there are no other corrections to the model becoming significant at lower photon numbers (e.g. accounting for higher levels of an artificial atom).

The last term in Eq. (2.37) has a dual nature. On one hand, the qubit frequency depends on the resonator population, giving rise to an ac Stark effect. On the other hand, the resonator frequency is qubit-state dependent, which can be used for readout purposes. Remarkably, the measurement provided by the dispersive Hamiltonian is quantum nondemolition (QND), owing to the fact that $\left[H_{\text {disp }}, \sigma_{z}\right] \approx 0$.

As anything, ability to readout the qubit state via the dispersive interaction comes with a price - reduced coherence time. It happens because the qubit state is entangled with the resonator state which has a loss channel through the transmission line. The resulting additional energy decay rate, refereed to as Purcell decay [PTP46], is

$$
\Gamma_{\text {Purcell }} \approx\left(\frac{g}{\Delta}\right)^{2} \kappa \text {. }
$$

\section{Principle of dispersive readout}

The dispersive readout scheme can be utilized to infer state of a superconducting qubit, and its optimization is an active field of research. The core question is how can the measurement SNR be maximized while minimizing back-action on a qubit?

Lets imagine that we can directly measure the field at the output of the lossless readout resonator dispersively coupled to a superconducting qubit. The measured quantity is a complex voltage of the microwave drive

$$
V_{\text {meas }}=\int_{0}^{\tau_{\mathrm{RO}}} V(t) d t
$$

corresponding to the output electric field itegrated over integration time $\tau_{\mathrm{RO}}$. Its real and imaginary parts are two non-commuting quadratures which are being measured simultaneously. These quadratures are refferred to as in-plane (I) and out-of-plane (Q). For a lossless readout resonator measured in reflection, the phase of the reflected signal contains information about the qubit state. 


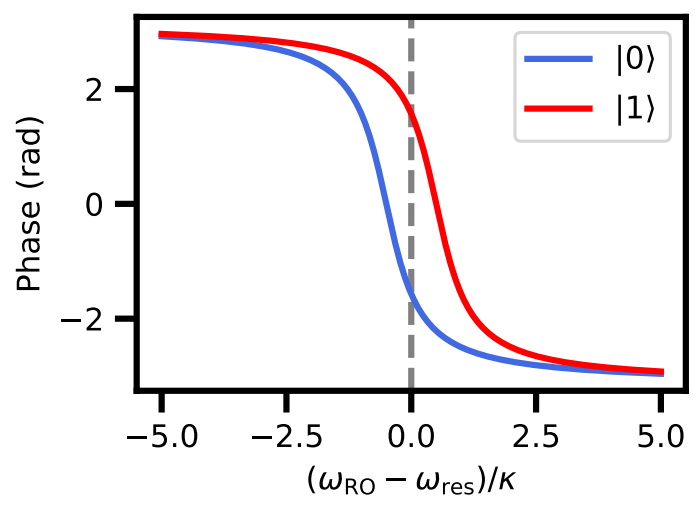

Figure 2.9: Phase of the signal reflected from the readout resonator for qubit being in ground (blue curve) and excited (red curve) state versus detuning of the readout tone $\omega_{\mathrm{RO}}$ from the bare resonator frequency $\omega_{\text {res }}$ indicated with grey dotted line. The phase responses are calculated for the optimal case $\chi=\kappa$, when the phase difference is maximum for $\omega_{\mathrm{RO}}=\omega_{\text {res }}$.

To demonstrate that, one needs to apply the IOT theory (Eqs. $(2.32,2.30)$ ) to the dispersive Hamiltonian (Eq. (2.37)). The output field (assuming long continuous drive) can be written as

$$
b_{\text {out }}=\left(-1+\frac{\kappa}{i\left(\omega-\omega_{0}-\chi\left\langle\sigma_{z}\right\rangle\right)_{q}+\kappa / 2}\right) b_{\text {in }} \text {, }
$$

where $\left\langle\sigma_{z}\right\rangle_{q}= \pm 1$ depends on the collapsed state of the qubit. While the absolute value of the output voltage is the same for both states, the phase is not, as can be seen in Fig. 2.9. Phase difference between the output voltages $\varphi_{\mathrm{eg}}=4 \arctan (\chi / \kappa)$ depends on the ratio of $\chi / \kappa$, and reaches its maximum of $180^{\circ}$ when $\chi=\kappa$. One of the benefits of $\mathrm{cQED}$ is that one can reliably implement big couplings, thus reaching this optimal angle is achievable for the majority of situations.

\section{SNR}

Signal-to-noise ratio is one of the most important measurement metrics. Focusing on the idealised case of direct measurement of a field at the readout resonator output, one can calculate a theoretical prediction for the SNR.

Assuming a readout pulse is applied to a resonator in the ground state, the output field evolves as

$$
\left.\beta(t)=\left(\beta_{\mathrm{i}}-\beta_{\mathrm{f}}\right) \exp \left[i\left(\omega_{\mathrm{RO}}-\omega_{\mathrm{res}}-\chi\left\langle\sigma_{z}\right\rangle_{q}\right)\right) t-\kappa t / 2\right]+\beta_{\mathrm{f}}
$$

where $\beta_{\mathrm{i}}=0$ and $\beta_{\mathrm{f}}$ are the initial and final pointer states calculated following Eq. (2.40). This evolution is shown in Fig. 2.10 for both qubit states. As expected, the output signal is delayed by $\tau_{\kappa} \sim 1 / \kappa$ due to the readout resonator ring-up/down time, affecting the timescale of the readout pulse variations and thus being crucial for minimizing the states discrimination times discussed in details in Sec. 4. For simplicity, here we focus on a standard regime highlighted in Fig. 2.10, when the integration time interval $\tau_{\text {RO }} \gg \tau_{\kappa}$ is chosen such that the output field is in the vicinity of the final steady-state, and $\tau_{\kappa}$ does not affect the average measured amplitude.

For further discussions, it is convenient to convert the output field in units of square root of measured photons $\sqrt{\bar{n} \kappa \tau_{\mathrm{RO}} / 4}$ leaving the cavity during the integration time. It is convenient to express the added Gaussian noise in these units, because it does not scale with time, and the evolution of pointer states can be described 
by considering only the mean value. The measured average output fields corresponding to different qubit states are separated by

$$
\left|V_{|1\rangle}-V_{|0\rangle}\right|=\sqrt{\bar{n} \kappa \tau_{\mathrm{RO}}} \sin \frac{\varphi_{01}}{2} .
$$

In units of measurement photons, the variation of quadratures due to quantum fluctuations is equal to

$$
\sigma_{\mathrm{QF}}=1 / 2
$$

Thus, in the ideal case of a lossless readout chain adding no noise to the signal, the measurement SNR is

$$
\mathrm{SNR}_{\text {ideal }}=\sqrt{\bar{n} \kappa \tau_{\mathrm{RO}}} \sin \frac{\varphi_{01}}{2} .
$$

In addition to SNR, another important quantity is the measurement fidelity

$$
F_{\text {meas }}=1-P(0 \mid 1)-P(1 \mid 0),
$$

where $P(0 \mid 1)$ is the conditional probability that after measuring qubit in state 1 , it is in fact in state 0 and $P(1 \mid 0)$ is the reverse. Assuming that qubit relaxation and higher-order effects do not affect the measurement and the steady-states are Gaussian, the measurement fidelity is related to SNR via

$$
F_{\text {meas }}=1-\operatorname{erfc}(\mathrm{SNR} / 2)
$$

where erfc is the error function. Similar to measurement fidelity, QNDness of the measurement can be characterized by measuring QND fidelity

$$
\mathrm{QND}_{\text {meas }}=\frac{P(0 \mid 0)+P(1 \mid 1)}{2} .
$$

For quantum information processing, $\mathrm{QND}_{\text {meas }}$ limits fidelity of the overall algorithm, and it is thus crucial that $\mathrm{QND}_{\text {meas }}$ is as close as possible to either 1 or 0 . If that is the case, the observer can be certain about the qubit state after the measurement, and can either move to the next algorithm steps when $\mathrm{QND}_{\text {meas }}=1$, or do so after flipping the qubit state for $\mathrm{QND}_{\text {meas }}=0$.
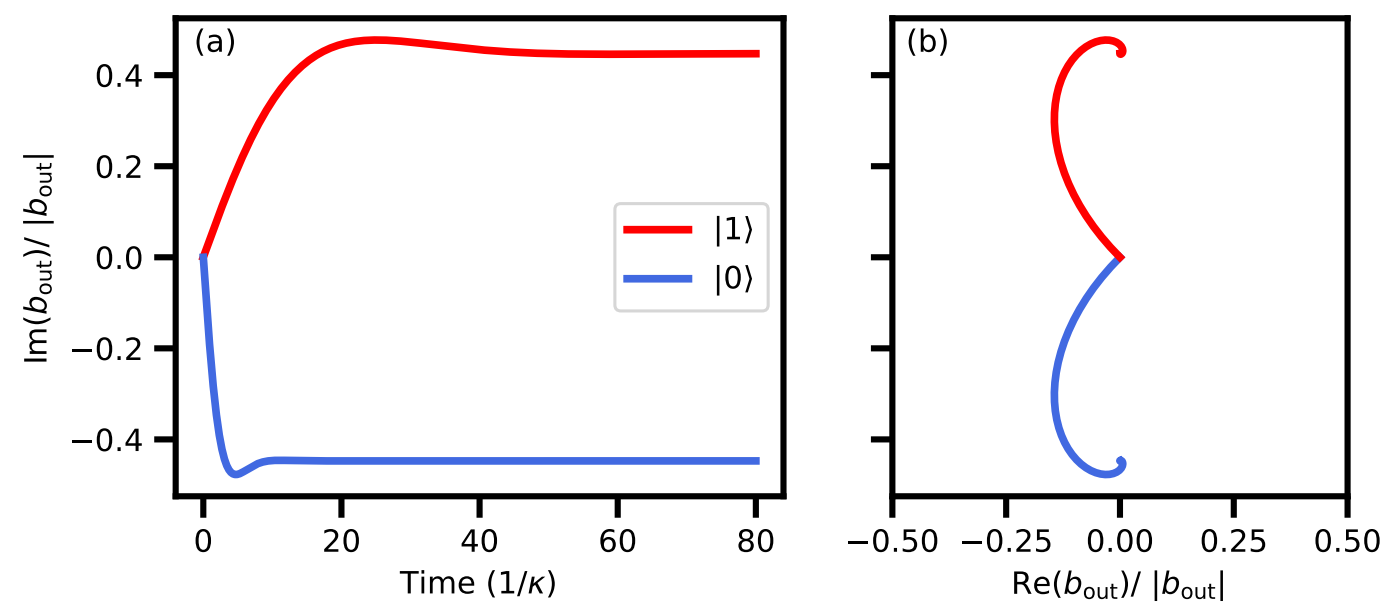

Figure 2.10: Evolution of the output field during the readout. The input pulse with field amplitude $b_{\text {in }}$ is applied at time $=0$ and is kept constant during the readout. (a) Imaginary part of the output field versus time for excited (red curve) and ground (blue curve) qubit states. (b) Parametric plot showing dynamics of the complex output field. 


\section{Measurement nonidealities and limitations}

The maximum achievable SNR scales as a square root of resonator photon population and integration time, and can be adjusted to the desired value by simply increasing these parameters. The increase, however, has its limits for both $\bar{n}$ and $\tau_{\text {RO }}$ because is causes a decrease of qubit lifetimes during the readout. This problem is most obvious for the measurement time when it approaches the qubit relaxation time $T_{1}$. Indeed, the qubit decay during readout is in fact contributing to the non-QNDness budget. If $\tau_{\text {RO }} \geq T_{1}$, QND fidelity (Eq. (2.47)) of the measurement decreases since the qubit has a significant chance to decay during the measurement.

A similar situation occurs when one wants to utilize high-power readout to boost SNR. The first bound for drive power comes from the critical photon number $n_{\text {crit }}$ which sets a limit on applicability for the dispersive approximation. Typically achievable $n_{\text {crit }}$ are higher than hundreds of photons, however, in practise, populating higher energy levels and lifetime decrease due to qubit state flips occurs for drives corresponding to $\bar{n} \ll n_{\text {crit }}$, as low as $\bar{n} \sim 1-10$ [JMS ${ }^{+} 12$; $\mathrm{SCK}^{+} 16$; $\mathrm{WKG}^{+} 17$; $\left.\mathrm{MMS}^{+} 19\right]$. This problem is not yet fully understood and is an object of theoretical and experimental investigation.

On top of these issues, SNR is also limited by the readout chain imperfections. The most crucial problem comes from the fact that the typical readout power is much smaller than the thermal noise of the room temperature apparatus corresponding to $\bar{n}_{\mathrm{RT}} \sim 1000$. Signal thus has to be amplified at cryogenic stages, and since any amplifier adds noise, the overall noise of the measured signal is higher than the quantum fluctuations ( Eq. (2.43)). The minimum possible noise of amplification chain can be achieved if the first amplifier in the chain is a quantum-limited amplifier [Cav82], i.e. adds only half a quanta of noise. The measured quadrature variation in that case is $\sigma_{\mathrm{QL}}=1 / \sqrt{2}$ in the units of square root of measured photons.

Another imperfection comes from losses $\eta_{\text {loss }}$ in coaxial cables, components and interconnects used for signal transmission, causing loss of information and thus lowering the SNR. As a result, Eq. (2.44) becomes

$$
\mathrm{SNR}=\sin \frac{\varphi_{01}}{2} \sqrt{\eta_{\text {loss }}\left(\frac{\sigma_{\mathrm{QL}}}{\sigma_{\text {meas }}}\right)^{2} \frac{\bar{n} \kappa \tau_{\mathrm{RO}}}{2}} .
$$

Here, the extra factor $1 / 2$ appears due to the noise added by the quantum-limited amplifier. Both propagation losses and non-quantum-limited amplification chain are typically accounted for by introducing quantum efficiency $\eta=\eta_{\text {loss }}\left(\frac{\sigma_{\mathrm{QL}}}{\sigma_{\text {meas }}}\right)^{2}$. For state-of-the-art experiments, typical values are $\eta=0.6 \pm 0.2\left[\mathrm{EKT}^{+} 19\right]$. If the SNR is small (SNR $<1$ ), only partial information about the quantum system can be inferred. Because of that, one needs to perform multiple readouts to extract information. For the majority of applications (e.g. quantum computing), however, one requires strong projective measurement resulting in a single-shot readout. It is thus important to be able to perform high-fidelity readout, implying:

- Fast readout in order to be able to react quickly to error syndromes measured in quantum error correction protocols, and ideally increase the coherence times of logical qubits.

- High SNR to suppress states separation error.

- QND readout to void uncontrollable qubit flips or, even worse, quantum system leaving the computational space.

To achieve this, one needs to implement a number of optimizations, with the most important one being utilization of a quantum-limited amplifier. 


\subsection{Josephson parametric amplifier}

Due to the typically low $(\bar{n}<10)$ readout powers $\left[\mathrm{JMS}^{+} 12 ; \mathrm{SCK}^{+} 16 ; \mathrm{WKG}^{+} 17 ; \mathrm{MMS}^{+} 19\right]$, in order to realize a single-shot readout of a superconducting qubit one needs a quantum-limited cryogenic amplifier. It can be realized by building a so-called parametric amplifier — a system with some parameter being varied, similar to a mechanical swing. For cryogenic microwave applications, the most successful approach is to use Josephson parametric amplifiers (JPAs): the inductance of the system (e.g. resonator) is being varied over time by utilizing Josephson junctions as nonlinear inductors.

In this section I describe basic concepts and operation principles of JPAs, as well as their important characteristics and how enhancement of the latter can be achieved when designing a device.

\subsubsection{Principles of parametric amplification}

The principle of parametric amplification can be demonstrated on a classical LC oscillator formed by a parallel-plate capacitor and a solenoid. Provided there are no losses, current flowing through the solenoid oscillates as

$$
I(t)=I_{0} \cos \omega_{0} t+\omega_{0} Q_{0} \sin \omega_{0} t,
$$

where $\omega_{0}=1 / \sqrt{L C}$ is the angular frequency of oscillations. The initial amplitudes of oscillations of current and charge $I_{0}$ and $Q_{0}$ correspond to two quadratures of the resonator state.

If one periodically partially inserts a ferromagnetic core inside the solenoid with angular frequency $\omega_{\mathrm{p}}=2 \omega_{0}$, such that the core is inside the solenoid when the current is maximum and is out when the current is zero, the resonance frequency becomes time-dependent: $\omega(t)=\omega_{0}+\delta \omega \cos \omega_{\mathrm{p}} t$ (assuming that $\delta \omega \ll \omega_{0}$ ). This modulation results in a dynamics of the system where the amplitude of the oscillating current gets amplified over time thanks to the energy being pumped into the resonator:

$$
I(t)=I_{0} e^{\delta \omega t / 2} \cos \omega_{0} t+\omega_{0} Q_{0} e^{-\delta \omega t / 2} \sin \omega_{0} t
$$

While one quadrature is being amplified, the other becomes squeezed. This is so-called phase-sensitive amplification, and the result of the amplification is a squeezed state [Aum20]. Since there is no coupling to other physical modes, phase-sensitive amplification is noiseless, provided that the resonator has no intrinsic losses.

Although seemingly being the best possible regime, phase-sensitive amplification is rarely useful in practice due to the fact that only one quadrature is amplified. Because of that, not only this amplification requires careful tuning, it is also susceptible to losses in coaxial cables (which degrade its efficiency), thus providing a minor increase of the measurement efficiency at a cost of making the measurement more complicated. As a result, this regime is typically used only when one starves for every last digit of quantum efficiency.

In order to amplify both quadratures and realize "phase-preserving" amplification, an extra degree of freedom has to be added, such that the 4D quadrature space is squeezed instead of the previously considered 2D. This can be achieved by coupling the "signal" resonator to another, so-called "idler" resonator. When pumping this system at $\omega_{\mathrm{p}}=\omega_{\mathrm{s}}+\omega_{\mathrm{i}}$, one can observe amplification of both quadratures of both signal and idler. The price to pay for this regime is the added noise. Because the signal mode is coupled to the idler mode, the idler vacuum noise contributes to the noise of the amplifier at the signal frequency. The added noise, provided a lossless system, is quantum limited [Cav82], i.e. is equal to half a photon. 


\subsubsection{Simplest realization of JPA}

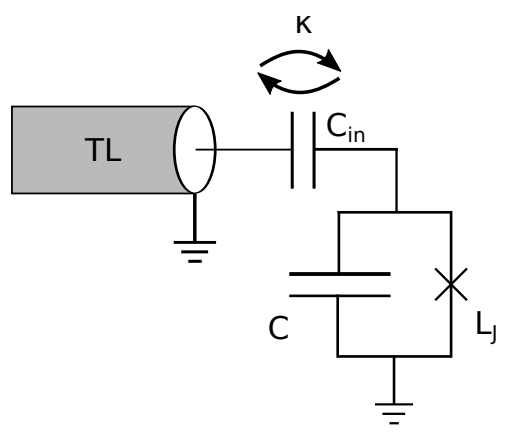

Figure 2.11: Schematic of the simplest realization of a JPA: a Josephson junction shunted with a capacitor C. The resulting nonlinear resonator is capacitively coupled to transmission line via capatitor $C_{\mathrm{in}}$.

In order to realize a microwave resonator with variable inductance, one can introduce a Josephson junction into the system or, even better, fully replace its inductance with a Josephson junction. This system has the same scheme and charge regime $E_{\mathrm{J}} \gg E_{\mathrm{C}}$ as a transmon qubit coupled to a transmission line, and hence can be described by the same Hamiltonian

$$
H=\hbar \omega_{0} a^{+} a+\hbar \frac{K}{2} a^{+} a^{+} a a .
$$

What makes a JPA different from a transmon qubit is the ratio of the coupling strength to the transmission line $\kappa$ and its Kerr coefficient $K$. When $\kappa \ll K$, each energy level can be individually addressed, and the system works as an artificial atom. In the other case, if $\kappa \gg K$, any applied drive excites a big amount of transitions, and the system can be treated as a classical nonlinear resonator - a resonator with photonnumber dependent resonance frequency. In order to operate a JPA as an amplifier, one needs to apply a strong pump tone which periodically varies inductance (and hence resonance frequency) of this system thanks to the JJ nonlinearity. A signal sent at the pumped JPA is then amplified.

To calculate the gain of a JPA and its response when applying the pump tone, one can use the IOT discussed in Sec. 2.2.2. For the ideal lossless case, the Langevin equation describing evolution of the intra-resonator field is

$$
\dot{A}=-i \omega_{0} A-i K A^{+} A A-\frac{\kappa}{2} A+\sqrt{\kappa} B_{\text {in }},
$$

with the input-output relation

$$
B_{\text {out }}=-B_{\text {in }}+\sqrt{\kappa} A
$$

Typically, there are two tones applied to the system: strong classical pump and weak quantum signal. We can then decompose input/output and inner fields into two parts, classical and quantum:

$$
\begin{array}{r}
B_{\text {in } / \text { out }}=\beta_{\text {in } / \text { out }} e^{i \omega_{\mathrm{p}} t}+b_{\text {in } / \text { out }}, \\
A=\alpha e^{i \omega_{\mathrm{p}} t}+a .
\end{array}
$$

Assuming that there is no pump depletion ( $\alpha$ does not depend on $b_{\text {in }}$ ), and given that the pump tone is much stronger than the signal( $|\alpha /\langle b\rangle| \gg 1)$, Eq. (2.52) can be dealt with by using a perturbation approach. First, we solve the classical response for the pump tone, and then linearize the equation for the signal response. 


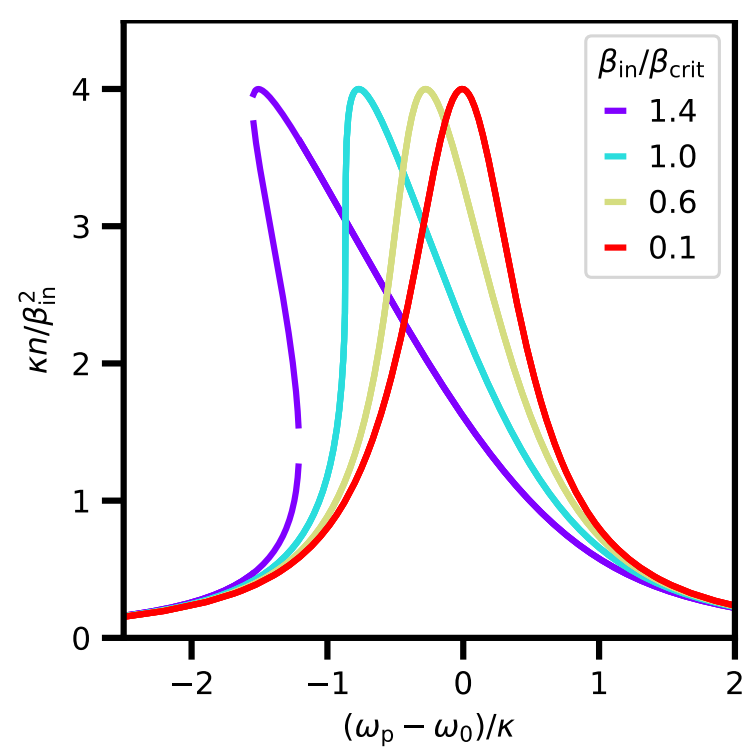

Figure 2.12: Normalized photon population as a function of pump frequency detuning shown for different pump powers.

\section{Classical nonlinear response}

For the classical nonlinear response, the Langevin equation is reduced to

$$
i\left(\omega_{\mathrm{p}}-\omega_{0}\right) \alpha-i K \alpha^{+} \alpha^{2}-\frac{\kappa}{2} \alpha=-\sqrt{\kappa} \beta_{\mathrm{in}} .
$$

Multiplied by its conjugate, this equation becomes

$$
\left[\left(\omega_{\mathrm{p}}-\omega_{0}\right)^{2}+\frac{\kappa^{2}}{4}\right] n-2\left(\omega_{\mathrm{p}}-\omega_{0}\right) K n^{2}+K^{2} n^{3}=\kappa\left|\beta_{\mathrm{in}}\right|^{2},
$$

where $n=|\alpha|^{2}$ is the resonator photon number. This is a cubic equation, and can be solved analytically. However, instead of deriving lengthy expressions, I present numerical results in Fig. 2.12, where the absolute value of inner-resonator field is shown for different pump powers. For a weak drive $\left(K|\alpha|^{2} \ll \kappa\right)$, the system demonstrates a Lorentian response. However, as pump power increases to the range where $K|\alpha|^{2} \sim \kappa$, the resonance frequency is Kerr-shifted to lower values and the curve gets tilted. At the critical value of $n$ referred to as bifurcation photon number

$$
n_{\mathrm{bif}}=\frac{\kappa}{\sqrt{27} K},
$$

derivative $\partial n / \partial \omega_{\mathrm{p}}$ at the frequency $\omega_{\mathrm{p}, \text { crit }}=\omega_{0}-\frac{\sqrt{3}}{2} \kappa$ is infinite, and the system response is extremely sensitive to the smallest changes in the input signal. For drive powers creating photon number population $n>n_{\text {bif }}$, Eq. (2.55) has three real solutions, two of which are stable. This bistable regime is called bifurcation, and has chaotic nature. For even higher drive powers, higher-order terms of the cosine expansion become significant giving rise to multistable solutions $\left[\mathrm{JBM}^{+} 14\right]$.

While being physically interesting, multistable regimes are not usually used for parametric amplification purposes (although this is possible, see e.g. [SVP ${ }^{+}$04; VDS09]). To operate a JPA, one typically utilizes resonator populations slightly lower than $n_{\text {bif }}$, profiting from the high sensitivity of the bifurcation point while being safe from chaotic behaviour of the bifurcation regime. 


\section{Signal response}

With the pump dynamics described in the previous section, it is now possible to use it to find the response to the weak quantum signal by following perturbation theory. Assuming $|a| \ll|\alpha|$, terms like $K \alpha a^{+} a$ can be neglected and we can consider a linear equation

$$
\dot{a}=-i \omega_{0} a-\frac{\kappa}{2} a-2 i K|\alpha|^{2} a-i K \alpha^{2} e^{-2 i \omega_{\mathrm{p}} t} a^{+}+\sqrt{\kappa} b_{\mathrm{in}} .
$$

Applying Fourier transform gives

$$
\left[i\left(\omega-\omega_{0}\right)-\frac{\kappa}{2}-2 i K|\alpha|^{2}\right] a(\omega)-i K \alpha^{2} a^{+}\left(2 \omega_{\mathrm{p}}-\omega\right)=-\sqrt{\kappa} b_{\mathrm{in}}(\omega) .
$$

The additional term at frequency $\omega_{\mathrm{i}}=2 \omega_{\mathrm{p}}-\omega$ (referred to as idler frequency) indicates that Eq. (2.57) describes a wave mixing process, and due to nonlinear interaction $a(\omega)$ and $a\left(\omega_{\mathrm{i}}\right)$ are coupled. This is closely related to what was discussed in Sec. 2.3.1: in order to amplify both quadratures of a signal, an additional mode has to be introduced.

Eq. (2.57) can be rewritten as two coupled equations:

$$
\left(\begin{array}{cc}
W(\omega) & -i K \alpha^{2} \\
i K\left(\alpha^{+}\right)^{2} & W^{*}\left(\omega_{\mathrm{i}}\right)
\end{array}\right)\left(\begin{array}{c}
a(\omega) \\
a^{+}\left(\omega_{\mathrm{i}}\right)
\end{array}\right)=-\sqrt{\kappa}\left(\begin{array}{c}
b_{\mathrm{in}}(\omega) \\
b_{\mathrm{in}}^{+}\left(\omega_{\mathrm{i}}\right)
\end{array}\right),
$$

where $W(\omega)=i\left(\omega-\omega_{0}-K|\alpha|^{2}\right)-\frac{\kappa}{2}$. After some algebraic steps, the input-output relation can be written as

$$
\left(\begin{array}{c}
b_{\text {out }}(\omega) \\
b_{\text {out }}^{+}\left(\omega_{\mathrm{i}}\right)
\end{array}\right)=\left(\begin{array}{cc}
g(\omega) & m(\omega) \\
m^{*}\left(\omega_{\mathrm{i}}\right) & g^{*}\left(\omega_{\mathrm{i}}\right)
\end{array}\right)\left(\begin{array}{c}
b_{\text {in }}(\omega) \\
b_{\text {in }}^{+}\left(\omega_{\mathrm{i}}\right)
\end{array}\right) .
$$

Here, the coefficients are

$$
\begin{array}{r}
g(\omega)=-1-\frac{\kappa W^{*}\left(\omega_{\mathrm{i}}\right)}{W(\omega) W^{*}\left(\omega_{\mathrm{i}}\right)-K^{2}|\alpha|^{4}} \\
m(\omega)=\frac{i \kappa K \alpha^{2}}{W(\omega) W^{*}\left(\omega_{\mathrm{i}}\right)-K^{2}|\alpha|^{4}} .
\end{array}
$$

Eq. (2.59) is the main result of these calculations, and will be a focus of further discussion throughout this section. To start things of, we will focus on one of the most important things - signal gain. As can be deduced, after interacting with the JPA the input field $b_{\text {in }}(\omega)$ gets multiplied by gain factor $g(\omega)$. In practice, it is more convenient to work with the power gain

$$
G(\omega)=\left|\frac{b_{\text {out }}(\omega)}{b_{\text {in }}(\omega)}\right|^{2}=|g(\omega)|^{2} .
$$

In Fig. 2.13(a), few examples of power gain frequency dependence are demonstrated. The curves are Lorentzians centered around corresponding pump frequencies. The maximal power gain $G_{0}$ (observed at pump frequency) can be adjusted by changing pump frequency and power to any desired value, up to more than $30 \mathrm{~dB}$. This tunability can be seen in Fig. 2.13(b), where the maximum of gain is shown as a function of pump parameters. Outside of the bifurcation area, represented with black in Fig. 2.13(a), the system is stable and can be used for parametric amplification. The grey dashed line indicates optimal pump frequency for each drive power, minimizing the required drive power required to obtain desired gain profile. 


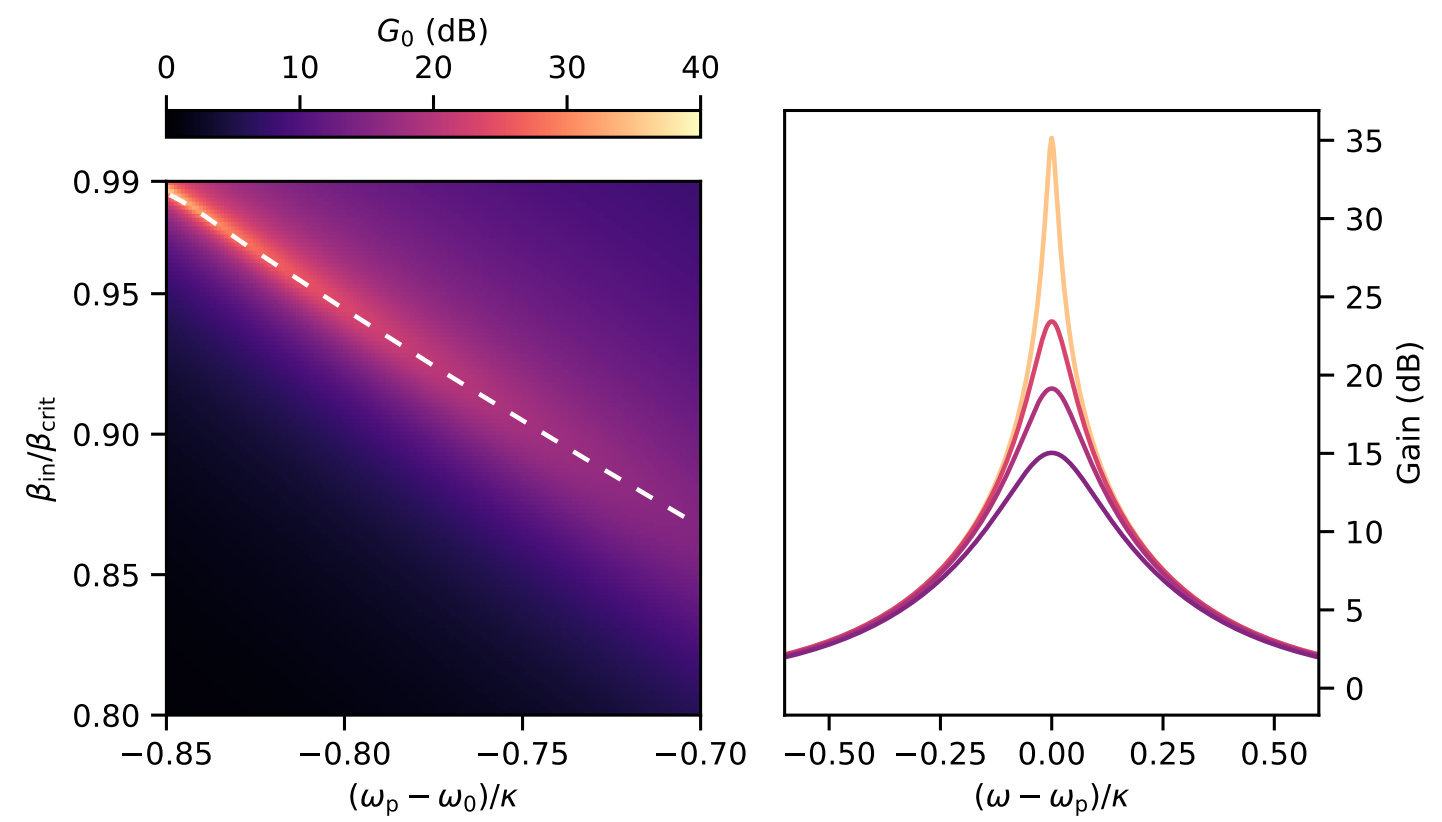

Figure 2.13: (a) Maximum of gain $G_{0}$ versus normalized pump power and frequency. White dotted line indicates optimal pump powers for each frequency. (b) Examples of gain profiles obtained for different optimal pump parameters.

One of the most important characteristics of any amplifier is instantaneous bandwidth defined as a frequency interval in which gain is less than $3 \mathrm{~dB}$ lower then the maximum gain. As is evident from Fig. 2.13(a), amplification band decreases with the maximum gain $G_{0}$ increase. The interdependence of these characteristics is described by the so-called gain-bandwidth product

$$
\mathrm{BW} \sqrt{G_{0}}=\kappa .
$$

Thus, increasing $G_{0}$ comes with a price, and operating at maximum gain is not always optimum.

Another important conclusion which can be made from Eq. (2.59) is that the output fields at signal and idler frequencies become entangled due to the off-diagonal term $m(\omega)$ representing idler gain. Depending on measurement procedure, this entanglement either results in generation of squeezed states, or becomes a source of the JPA noise, caused by the quantum noise at the idler frequency being added to the output signal tone via term $m(\omega) b_{\text {in }}^{+}\left(\omega_{\mathrm{i}}\right)$. Before explaining these phenomena in details in the next subsections, it is important to mention some relations between $g(\omega)$ and $m(\omega)$ originating from the fact that photons are Bose particles and thus obey

$$
\begin{array}{r}
{\left[b_{\text {in } / \text { out }}\left(\omega_{1}\right), b_{\text {in/out }}^{+}\left(\omega_{2}\right)\right]=\delta_{\omega_{1}, \omega_{2}}} \\
\left.\left[b_{\text {in/out }}\left(\omega_{1}\right), b_{\text {in/out }}\left(\omega_{2}\right)\right]\right]=0 .
\end{array}
$$

By using these relations, one can find that

$$
\begin{gathered}
|g(\omega)|^{2}-|m(\omega)|^{2}=1, \\
g(\omega) m\left(\omega_{\mathrm{i}}\right)=g\left(\omega_{\mathrm{i}}\right) m(\omega) .
\end{gathered}
$$

In the high gain limit $|g|^{2} \gg 1$ (the typically used power gains are $\left.15-25 \mathrm{~dB}\right),|g(\omega)| \approx|m(\omega)|$. As a result, output powers at signal and idler frequencies are equal, and idler tone carries the same information signal 
does. Additionally, one can deduce that $|g(\omega)|^{2}=\left|g\left(\omega_{\mathrm{i}}\right)\right|^{2}$, meaning that the power gain curve is symmetric with respect to the pump frequency (provided there are no losses present in the system).

\subsubsection{Important characteristics of JPAs}

Although JPAs posses interesting physics by themselves, their main attractiveness comes from the fact that they can be used as a first amplifier in the chain, adding minimum amount of noise allowed by quantum mechanics. They should thus be thought of as a practical device, possessing a set of characteristics of interest which should be optimized when designing and operating an amplifier. Here, one can find a detailed discussion for the most important specifications.

\section{Added noise}

The most important figure of merit for a JPA is its added noise. As will be shown, a JPA is a quantum-limited amplifier, and it is placed in the amplification chain only to make it's noise quantum limited. It is thus important to understand the origin of the JPA added noise, and estimate the power gain required to suppress noise of the next amplifier in the chain.

(a)

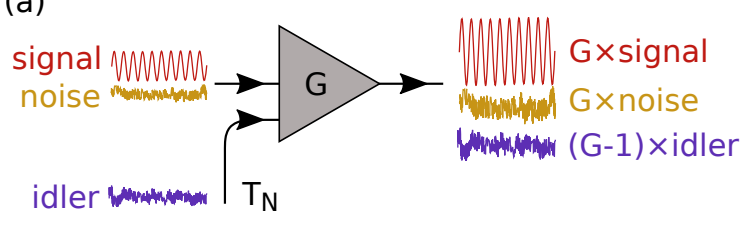

(b)

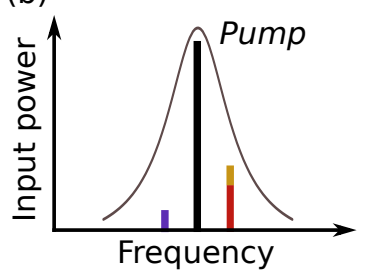

(c)

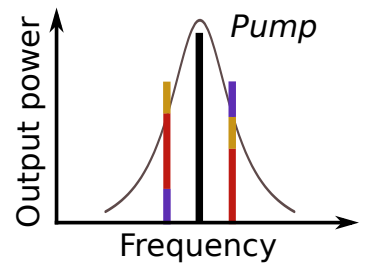

Figure 2.14: Sketch illustrating origin of JPA noise in phase-preserving regime. (a) Schematic of a signal (red) and associated noise (orange) amplified by an amplifier with gain $G$ and corresponding noise temperature $T_{\mathrm{N}}$. (b), (c) Power spectrum of input and out fields, respectively. Lorentzian curves indicate signal power gain profile.

When operated in phase-preserving regime, meaning that only signal tone is being measured while $\omega \neq \omega_{\mathrm{i}}$, JPA adds quantum-limited noise [Cav82]. It happens due to the fact that quantum fluctuations present at the idler tone are added to the output signal tone, which is sketched in Fig. 2.14. Following Eq. (2.59), the input field $b_{\text {in }}(\omega)$ is amplified, and also "copied" to the idler output field due to the term $m^{*}\left(\omega_{\mathrm{i}}\right) b_{\text {in }}(\omega)$. Since in the high-gain limit $|g(\omega)| \approx\left|g\left(\omega_{\mathrm{i}}\right)\right|, b_{\text {in }}(\omega)$ contributes equally to the output power of signal and idler tones. The same is true for the idler tone: the input idler field also gets amplified and "copied" to the output signal tone, with the same power gain. Although nothing is usually sent at the idler frequency, there are always quantum fluctuations with power spectral density $S(\omega)=\frac{\hbar \omega}{2} \operatorname{coth}\left(\frac{\hbar \omega}{2 k_{\mathrm{B}} T}\right)$, which get transferred to the signal tone due to wave mixing processes. In the units of square root of measured photons, the added noise corresponds to $\sigma_{\text {added }}=1 / 2$, increasing the total noise of the measurement to $\sigma_{\text {total }}=\sqrt{\sigma_{\text {added }}^{2}+\sigma_{\text {fluctuations }}^{2}}=1 / \sqrt{2}$.

Typically, cQED experiments require several amplification stages since the gain of $\sim 100 \mathrm{~dB}$ needed to make signals detectable by the measurement apparatus is hard (if not impossible) to achieve by using a single 

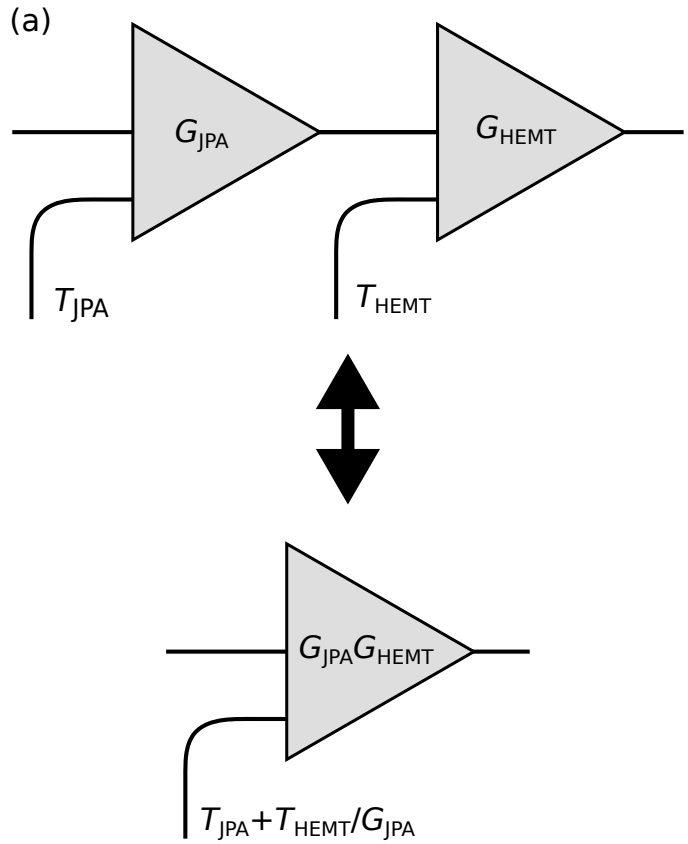

(b)

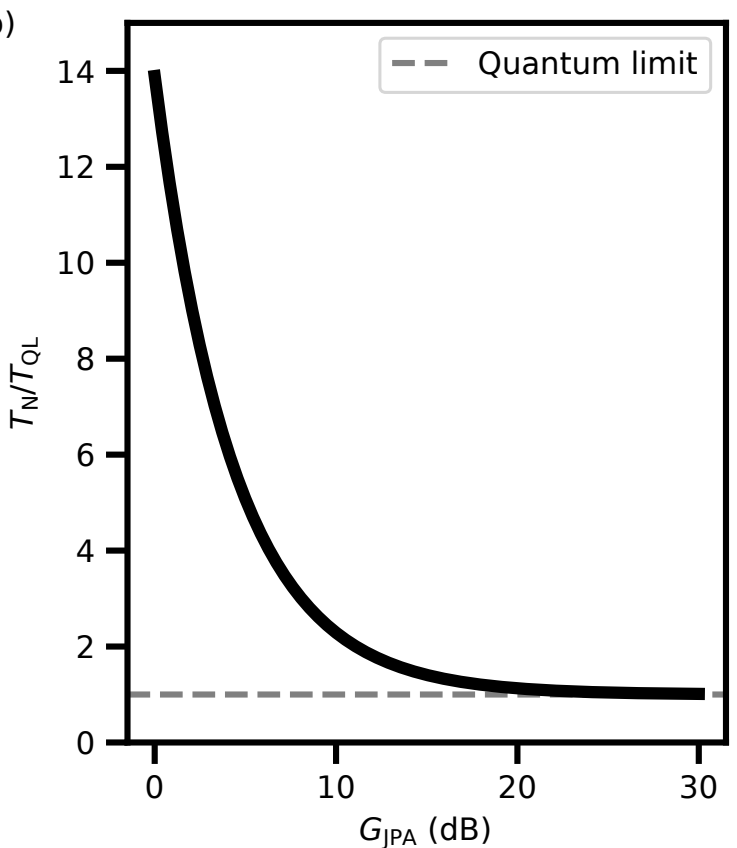

Figure 2.15: (a) Schematic of the amplification chain consisting of JPA and HEMT(top image), which can be thought of as a single amplifier with effective gain $G_{\text {JPA }} G_{\text {HEMT }}$ and noise temperature $T_{\text {JPA }}+T_{\text {HEMT }} / G_{\text {JPA }}$. (b) Added noise temperature of the amplification chain in the units normalized by quantum-limited noise as a function of JPA power gain. The noise temperature of HEMT was considered to be $2 \mathrm{~K}$. The grey dotted line indicates quantum-limited noise.

amplifier. As depicted in Fig. 2.15(a), the amplification chain consisting of a series of amplifiers with gains $G_{i}$ and noise temperatures $T_{i}$ in the abscense of losses can be modelled as an single equivalent amplifier with gain $G=\prod_{i} G_{i}$ and noise temperature $T=T_{1}+\frac{T_{2}}{G_{1}}+\frac{T_{3}}{G_{1} G_{2}}+\ldots$. When building an amplification chain, it is thus important to put the lowest-noise amplifiers first, and make sure that gain of each amplifier is big enough to suppress noise of the next amplifier in the chain, thus limiting overall noise of the chain to the noise of the first amplifier. For JPAs it is thus important to estimate gain required to suppress noise of the second amplifier in the chain, which is usually a HEMT amplifier with noise temperature of $2-4 \mathrm{~K}$. In Fig. 2.15(b), the noise temperature of the amplification chain is shown as a function of the JPA gain. As is evident, the noise temperature approaches quantum limit at $15 \mathrm{~dB}$, and increasing gain above $25 \mathrm{~dB}$ does not improve the noise characteristics. Because of that, the typically chosen gain value is $20 \mathrm{~dB}$.

\section{Losses}

So far only lossless schemes were considered. However, since losses are the main factor limiting the overall JPA performance, it is important to be able to estimate their influence and low they should be kept.

Similar to the treatment for resonators with finite $Q_{\mathrm{i}}$ (see Sec. 2.2.2), to model losses one can introduce a bath with Hamiltonian $H_{l}=\int_{R} d \omega \hbar \omega l^{+}(\omega) l(\omega)$ coupled to the JPA with coupling strength $\sqrt{\frac{\gamma}{2 \pi}}$. The Langevin equation (2.52) corresponding to this system is

$$
\dot{a}=-\frac{i}{\hbar}\left[a, H_{\mathrm{sys}}\right]-\frac{\kappa+\gamma}{2} a(t)-\sqrt{\kappa} b_{\text {in }}(t)-\sqrt{\gamma} l_{\text {in }}(t) .
$$

The output field $b_{\text {out }}(\omega)$ inferred from Eq. (2.59) then changes to

$$
b_{\text {out }}(\omega)=g_{\gamma}(\omega) b_{\text {in }}(\omega)+m_{\gamma}(\omega) b_{\text {in }}^{+}\left(\omega_{\mathrm{i}}\right)+\sqrt{\frac{\gamma}{\kappa}}\left(g_{\gamma}(\omega)+1\right) l_{\text {in }}(\omega)+\sqrt{\frac{\gamma}{\kappa}} m_{\gamma}(\omega) l_{\text {in }}^{+}\left(\omega_{\mathrm{i}}\right),
$$


where $g_{\gamma}, m_{\gamma}$ are calculated using Eq. (2.61) with $W(\omega)$ being changed to to $W_{\gamma}(\omega)=i\left(\omega-\omega_{0}-K|\alpha|^{2}\right)-$ $\frac{\kappa+\gamma}{2}$.

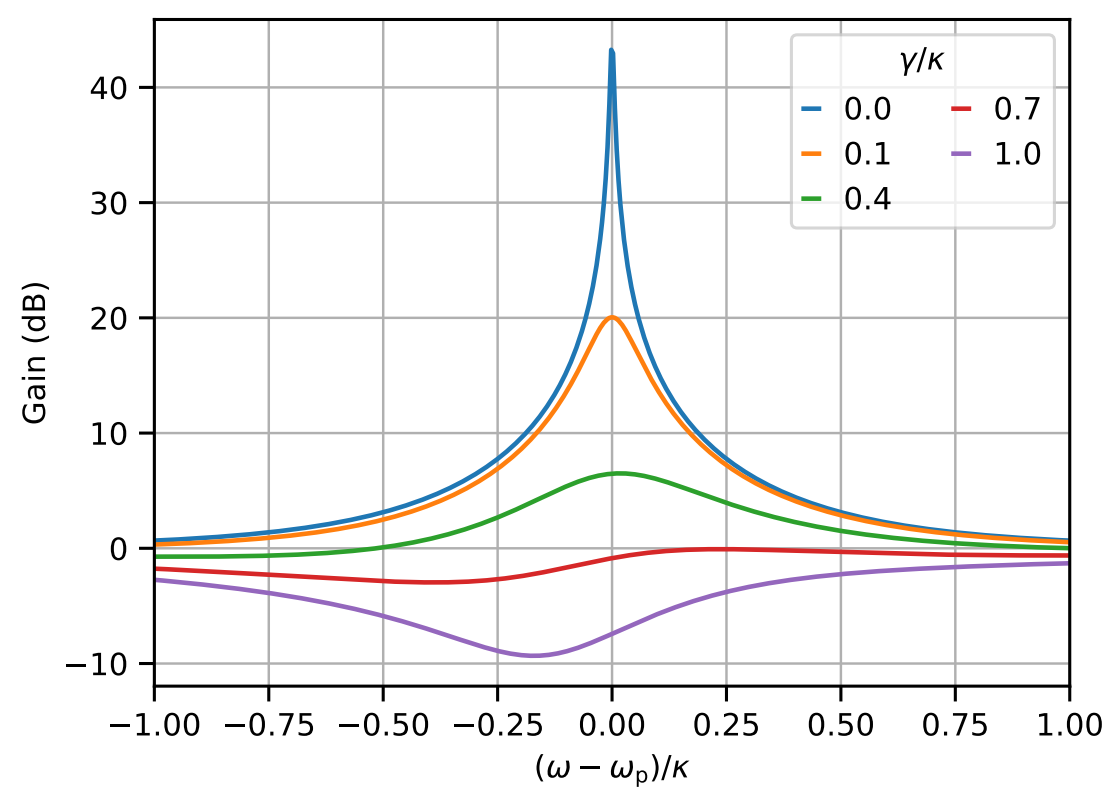

Figure 2.16: Gain versus normalized frequency for different loss rates. Pump frequency is the same for all plots $\left(\omega_{\mathrm{p}}=\omega_{0}-0.86 \kappa\right)$ while pump power was adjusted for each curve to maximize $G_{0}$.

There are two negative effects caused by losses. First, they change the gain curve making it asymmetric and limiting the maximum achievable gain, as illustrated in Fig. 2.16. Secondly, the added noise of JPA increases due to interaction of the signal tone with two additional modes, $l_{\text {in }}(\omega)$ and $l_{\text {in }}^{+}\left(\omega_{\mathrm{i}}\right)$. In the limit of high gain,

$$
T_{\text {extra }} \approx \frac{2 \gamma}{\kappa} \frac{\hbar \omega}{2}=\frac{2 \gamma}{\kappa} T_{\mathrm{QL}}
$$

where $T_{\text {extra }}$ is the additional added noise.

For a quantum-limited JPA it is thus essential that $\kappa \gg \gamma$ (or, in other terms, $Q_{c} \ll Q_{i}$ ). Conveniently, JPAs are usually broadband and utilize $Q_{\mathrm{c}}=10-100$. The required $Q_{\mathrm{i}} \geq 10^{3}$ is hence easily achievable, which makes JPAs relatively insensitive to fabrication limitations and numerous effects which could limit $Q_{\mathrm{i}}$, such as e.g. dielectric or quasiparticle losses.

\section{Signal-pump detuning}

When using a reflection-based amplifier, to separate input and output fields one uses a circulator [Poz11] - directional passive device which transmits signals in the forward direction, while strongly attenuating reverse-propagating signals. The attenuation, however, is typically limited to $20 \mathrm{~dB}$ while the pump power is 20-40 dB higher than signal. Because of that, when using a JPA for qubit readout, one has to be careful about pump leaking onto the qubit chip through the circulator isolation. Due to the leakage, there appears an additional strong drive influencing the state of the measured system. For the discussed JPA schematic (cf. Fig. 2.11), the pump tone is detuned from the signal by (at best) few tens of $\mathrm{MHz}$, and pump leakage becomes a huge problem. 
To combat that, one could introduce several isolators between the qubit chip and the JPA. However, they are bulky elements relying on magnetic elements. Not only they create unwanted magnetic fields, they also introduce additional losses thus lowering quantum efficiency. It would thus be beneficial to avoid using unneeded isolators by increasing the pump-signal detuning to at least hundreds of $\mathrm{MHz}$. By doing so, the pump-induced photon population in the qubit's readout resonator becomes negligible due to its Lorentzian response with typical linewidth of $1-10 \mathrm{MHz}$.

There is a number of ways to achieve this detuning. First, one could use three-wave mixing nonlinearity, thus changing relation between mixed frequencies to $\omega_{\mathrm{p}}=\omega_{\mathrm{s}}+\omega_{\mathrm{i}}$. Pump frequency is then approximately twice as big as the signal one. This regime can be achieved by using flux-pumping of dc-SQUIDs, or utilizing the controllable nonlinearities of rf-SQUIDs [Zor19] or SNAILs [FVS ${ }^{+} 17$; $\mathrm{FSL}^{+} 18$; $\mathrm{SFJ}^{+}$19; $\mathrm{SSL}^{+}$20]. Another solution is to use a system of two coupled nonlinear resonators, which can operate in a non-degenerate regime for which pump and signal tones occupy different physical modes and gain has a double-Lorentzian profile. Non-degenerate amplification can be realized for both 3-wave-mixing $\left[\mathrm{BSM}^{+} 10 ; \mathrm{RFN}^{+} 12 ; \mathrm{LCC}^{+} 17\right]$ and 4-wave-mixing $\left[\mathrm{ESM}^{+} 14\right.$; $\left.\mathrm{WTR}^{+} 20\right]$ regimes (a detailed discussion about the 4-wave-mixing nondegenerate amplifier developed during my $\mathrm{PhD}$ thesis can be found in chapter 3).

\section{Saturation power}

For any amplifier, the physical process of amplification can be described as transfer of energy from pump into the signal of interest. Naturally, there is a limit of energy transfer rate, and for input signal power "demanding" higher transfer rates that $G P_{\text {in }}>P_{\text {limit }}$, the apparent power gain is lower than the expected one. This effect results in gain being signal-power-dependent as it reaches a certain threshold. The threshold is referred to as saturation power or $1 \mathrm{~dB}$ compression point, and is defined as a signal power at which gain decreases by $1 \mathrm{~dB}$.

For JPAs utilizing fourth order nonlinearity, the main saturation mechanisms are pump depletion (reduction of pump power due to amplification process) and Stark shift of the JPA resonance frequency induced by signal [ $\left.\mathrm{FSL}^{+} 18 ; \mathrm{PDM}^{+} 19\right]$. Both effects change pumping parameters: the first one reduces pump photon number population, and the second changes the pump tone detuning $\omega_{\mathrm{p}}-\omega_{0}$. This results in a decrease of gain of an optimally pumped JPA, as can be deduced from Fig. 2.13(b).

For a four-wave-mixing amplifier, the Stark shift effect is the dominant source of saturation. Following $\left[\mathrm{FSL}^{+} 18\right]$, the corresponding $1 \mathrm{~dB}$-compression point obeys

$$
P_{1 \mathrm{~dB}}^{\mathrm{Stark}} \sim \frac{\kappa^{2}}{K} \frac{\hbar \omega_{0}}{G_{0}^{5 / 4}}
$$

Increase of $P_{\text {sat }}$ is an important part of the amplifier development. As evident from Eq. (2.69), it can be achieved by increasing $\kappa$ and/or decreasing Kerr nonlinearity.

The resonator bandwidth cannot be increased to arbitrary big value. This is caused by the fact that current induced by pump tone should not exceed critical current of the Josephson junction, which entails that

$$
p Q \geq 16 / \sqrt{3},
$$

where $p=\frac{L_{\mathrm{J}}}{L+L_{\mathrm{J}}}$ is the nonlinear inductance participation ratio. Thus, the highest safe value for $\kappa$ is $\omega_{0} / 10$.

For Kerr suppression, the optimal choice suggested in [EW14] is to replace a single Josephson junction with an array, distributing power among several junctions. For the scheme shown in Fig. 2.11, if a single 
junction with inductance $L_{\mathrm{J}}$ is replaced with $N$ junctions and reducing capacitance to $C / N$ to maintain the same resonance frequency, the Kerr coefficient scales [SSL $\left.{ }^{+} 20\right]$ as $K \propto 1 / N$. A complementary Kerr decrease, achievable by increasing critical current of the Josephson junction, is limited by Eq. (2.70), because participation ratio starts to lower due to unavoidable linear inuctances. In practice, the typically used critical currents are $I_{\mathrm{c}}=1-10 \mathrm{\mu s}$.

Saturation power can be further increased by utilizing other wave-mixing regimes while fully suppressing forth order nonlinearity. It was shown in $\left[\mathrm{FSL}^{+} 18\right]$ that when using SNAILs for three-wave-mixing Kerrfree amplifiers, saturation power is limited by pump depletion requiring an order of magnitude higher signal powers than signal-induced Stark shift.

\section{Bandwidth}

One of the most important characteristics of an amplifier is instantaneous bandwidth defined as a frequency interval in which gain is less than $3 \mathrm{~dB}$ lower then the maximum gain. For non-degenerate 4-wave-mixing amplifiers, it is limited to about $1 \%$ of the resonance frequency by gain-bandwidth product (Eq. (2.63)) and critical current of Josephson junction (Eq. (2.70)). The resulting band of few tens of $\mathrm{MHz}$ is sufficient for a single-qubit readout since it is bigger that the typical readout resonator linewidth of $1-10 \mathrm{MHz}$ and also allows to resolve pulses with the typical length of few tens of nanoseconds.

However, there are application requiring wider band, such as multiplexed readout of several qubits or kinetic inductance detector arrays, not to mention the much desired user-friendliness of a 'plug-and-play' device with a band of couple GHz. For JPAs, the gain-bandwidth product has to be dealt with to widen the band. There are currently two approaches: changing the product and getting rid of it by not using resonator-based structures.

Gain-bandwidth product can be changed by using an impedance matching technique $\left[\mathrm{MWB}^{+} 14 ; \mathrm{RKC}^{+}\right.$; NFE17; YGS ${ }^{+} 20$ ], realized by making coupling $\kappa(\omega)$ frequency-dependent by introducing resonator-based schemes in front of the JPA. In principle, if coupling was a complex function of frequency $\kappa(\omega)$, this function could be engineered to compensate the detuning $\omega-\omega_{0}$ in Eq. (2.61), thus making the gain profile flat over a wide frequency range. If $\kappa(\omega)$ is accordingly designed, the gain-bandwidth product (2.63) changes, resulting in $\mathrm{BW} \approx \kappa$. Thus, an impedance-matched JPA can reach an instantaneous band of hundreds of MHz.

Another solution for the band increase is to get rid of the resonator, and use nonlinear transmission line as a wave-mixing media. This kind of amplifier is called a Traveling Wave Parametric Amplifier(TWPA) and is a widely used approach in optics [Agr13]. First reported cryogenic microwave TWPAs were based on kinetic inductance [HEDLZ12]. Unfortunately, even nowadays $\left[\mathrm{MVW}^{+} 21\right]$ they are still not optimal for qubit readout applications due to high pump powers, gain profile with many ripples, and non-quantum-limited noise. A Josephson-junction-based TWPA was first proposed in [YFMS], where the nonlinear media is a lumpedelement transmission line with inductances replaced by Josephson junctions. Nowadays there are many different realizations of Josephson junction TWPAs, utilizing different wave mixing regimes[ $\left.\mathrm{MOH}^{+} 15 ; \mathrm{MM} 19\right]$, phase matching realizations[OMSZ; $\mathrm{PRD}^{+} 20 ; \mathrm{REP}^{+}$21], and pumping schemes [Zor19]. Despite having a few-GHz bandwidth and high saturation power, nowadays TWPAs are not yet quantum-limited, most probably due to dielectric losses $\left[\mathrm{OAB}^{+} 08\right]$ and sidebands generation $\left[\mathrm{PNW}^{+} 21\right]$. For summary about TWPAs' state of the art, the reader is redirected to [ERPR21]. 


\section{Dimer Josephson Junction Array Amplifier (DJJAA)}

The Dimer Josephson Junction Array Amplifier (DJJAA) [WTR $\left.{ }^{+} 20\right]$ is a 4-wave-mixing non-degenerate parametric amplifier. Owing to the design based on a Josephson junction array (JJA), the DJJAA saturation power exceeds thousand photons per $\mu$ s making this amplifier suitable for experiments requiring high-power readout. Non-degeneracy is achieved by engineering the JJA dispersion, ensuring few hundreds of $\mathrm{MHz}$ of detuning between pump and signal frequencies. Last but not least, the amplifier can be fabricated with a widely-accessible two-step optical lithography.

This chapter is organized as follows. First, the operating principle of a 4-wave-mixing non-degenerate amplifier is discussed (Sec. 3.1). Then, the DJJAA design concept and dispersion engineering are presented in Sec. 3.2, followed by the experimental realization of the DJJAA and its characterization (Sec. 3.3). Sec. 3.4 is dedicated to the utilization of superconducting qubits as power detector, focusing on accurate calibration of DJJAA saturation power.

\subsection{Theoretical model}

This section is a brief summary of a theoretical model used to predict the response of a 4-wave-mixing non-degenerate amplifier. Selected simulations which can help to build intuition on how to design and operate such an amplifier are shown. For a detailed discussion of the model, the interested reader is referred to $\left[\mathrm{ESM}^{+} 14\right]$.

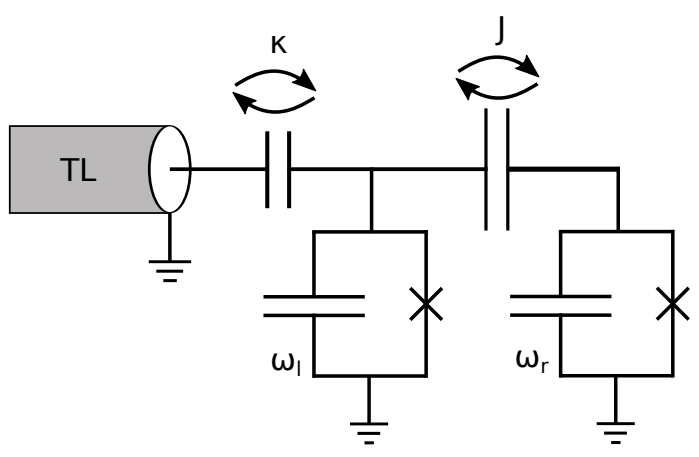

Figure 3.1: Equivalent schematic of a JPD.

To achieve non-degenerate four-wave-mixing amplification, one can use a system consisting of two modes $\left(\omega_{+} \neq \omega_{-}\right)$which are both coupled to a transmission line and have a cross-Kerr interaction $K_{\text {cross }} a_{+}^{+} a_{+} a_{-}^{+} a_{-}$ between each other. This system is further referred to as dimer, and the corresponding Josephson junctionbased parametric amplifier - as Josephson Parametric Dimer (JPD). For algebraic reasons, it is convenient to map such system to the Bose-Hubbard model [SCWS08]. The equivalent circuit of a Bose-Hubbard JPD is shown in Fig. 3.1. There are two nonlinear modes with frequencies $\omega_{1}, \omega_{\mathrm{r}}$ coupled with a coupling 
strength $J$. One of the modes, denoted left, is coupled to a transmission line with a coupling strength $\kappa$. All intrinsic losses of the dimer are accounted for by introducing loss rate $\gamma$ for the right resonator. This system is described by the Hamiltonian

$$
H / \hbar=\omega_{\mathrm{l}} A_{1}^{+} A_{\mathrm{l}}+\omega_{\mathrm{r}} A_{\mathrm{r}}^{+} A_{\mathrm{r}}+J\left(A_{\mathrm{l}} A_{\mathrm{r}}^{+}+A_{\mathrm{r}} A_{1}^{+}\right)+\frac{1}{2}\left(K_{1} A_{\mathrm{l}}^{+} A_{1}^{+} A_{1} A_{\mathrm{l}}+K_{\mathrm{r}} A_{\mathrm{r}}^{+} A_{\mathrm{r}}^{+} A_{\mathrm{r}} A_{\mathrm{r}}\right),
$$

where $K_{1 / \mathrm{r}}$ are the modes' Kerr coefficients, and $A_{1 / \mathrm{r}}$ is the annihilation operator for the corresponding resonator.

Due to the mixing term $J\left(A_{1} A_{\mathrm{r}}^{+}+A_{\mathrm{r}} A_{1}^{+}\right)$, left and right modes hybridize, resulting in symmetric and asymmetric modes with eigenfrequencies

$$
\omega_{ \pm}=\frac{\omega_{1}+\omega_{\mathrm{r}}}{2} \pm \sqrt{J^{2}+\left(\frac{\omega_{1}-\omega_{\mathrm{r}}}{2}\right)^{2}}
$$

and corresponding linewidths

$$
\kappa_{ \pm}=\frac{\kappa}{2}\left(1 \pm \frac{\left|\omega_{1}-\omega_{\mathrm{r}}\right|}{\sqrt{4 J^{2}+\left(\omega_{l}-\omega_{\mathrm{r}}\right)^{2}}}\right) .
$$

For the optimal hybridization case of $\omega_{1}=\omega_{\mathrm{r}}$, the modes' frequencies are split by $2 J$, and the effective coupling strength to the transmission line of each hybridized mode is $\kappa / 2$ (photons spend equal amount of time in left and right resonators).

Similar to Sec. 2.3.2, annihilation operators $A_{1 / \mathrm{r}}=\alpha_{1 / \mathrm{r}} e^{i \omega_{\mathrm{p}} t}+a_{\mathrm{l} / \mathrm{r}}$ are decomposed into strong classical ( $\left.\alpha_{1 / \mathrm{r}} e^{i \omega_{\mathrm{p}} t}\right)$ and quantum $\left(a_{\mathrm{l} / \mathrm{r}}\right)$ parts. Equations of motion for the classical part describe the system response to the pump tone. They are given by Langevin equations, leading to a closed set of coupled nonlinear equations

$$
\begin{array}{r}
\left(i\left(\omega_{\mathrm{p}}-\omega_{\mathrm{l}}\right)-\frac{\kappa}{2}\right) \alpha_{1}-i J \alpha_{\mathrm{r}}-i K_{1} \alpha_{\mathrm{l}}^{+} \alpha_{1}^{2}+\sqrt{\kappa} \beta_{\text {in }}=0 . \\
\left(i\left(\omega_{\mathrm{p}}-\omega_{\mathrm{r}}\right)-\frac{\gamma}{2}\right) \alpha_{\mathrm{r}}-i J \alpha_{\mathrm{r}}-i K_{\mathrm{r}} \alpha_{\mathrm{r}}^{+} \alpha_{\mathrm{r}}^{2}=0 .
\end{array}
$$

These equations, depending on pump frequency and power, have either one or multiple solutions. The latter results in a chaotic behaviour, and is typically avoided for parametric amplification purposes.

Following the procedure discussed in Sec. 2.3.2, weak quantum response can be calculated once classical fields are known. Assuming there is no pump depletion, the Langevin equations for quantum signal linking annihilation operators of left and right modes at signal $\omega$ and idler $\left(\omega_{i}=2 \omega_{p}-\omega\right)$ frequencies with transmission line fields are (for details, see supplementary of Ref. [ESM $\left.{ }^{+} 14\right]$ ):

$$
a(\omega)=\sqrt{\kappa} V b_{\text {in/out }}(\omega),
$$

where $a(\omega)=\left(a_{1}(w), a_{1}^{+}\left(\omega_{\mathrm{i}}\right), a_{\mathrm{r}}(\omega), a_{\mathrm{r}}^{+}\left(\omega_{\mathrm{i}}\right)\right)^{\mathrm{T}}, b_{\text {in/out }} \approx\left(b_{\text {in/out }}(\omega), b_{\text {in/out }}^{+}\left(\omega_{\mathrm{i}}\right), 0,0\right)^{\mathrm{T}}$, and

$$
V=\left(\begin{array}{cccc}
i\left(\omega-\omega_{1}\right)-\kappa / 2 & -i K_{1} \alpha_{1}^{2} & -i J & 0 \\
i K_{1} \alpha_{1}^{* 2} & -i\left(\omega_{\mathrm{i}}-\omega_{\mathrm{l}}\right)-\kappa / 2 & 0 & i J \\
-i J & 0 & i\left(\omega-\omega_{\mathrm{r}}\right)-\gamma / 2 & -i K_{\mathrm{r}} \alpha_{\mathrm{r}}^{2} \\
0 & i J & i K_{\mathrm{r}} \alpha_{\mathrm{r}}^{* 2} & -i\left(\omega_{\mathrm{i}}-\omega_{\mathrm{r}}\right)-\gamma / 2
\end{array}\right) .
$$


By using the Input-Output relation, the output field at signal frequency can be written as

$$
b_{\text {out }}(w)=g(\omega) b_{\text {in }}(\omega)+m(\omega) b_{\text {in }}^{+}\left(\omega_{\mathrm{i}}\right),
$$

with the signal and idler gains equal to

$$
\begin{array}{r}
g(\omega)=-1-\kappa V_{11}^{-1}(\omega), \\
m(\omega)=-\kappa V_{12}^{-1}(\omega) .
\end{array}
$$
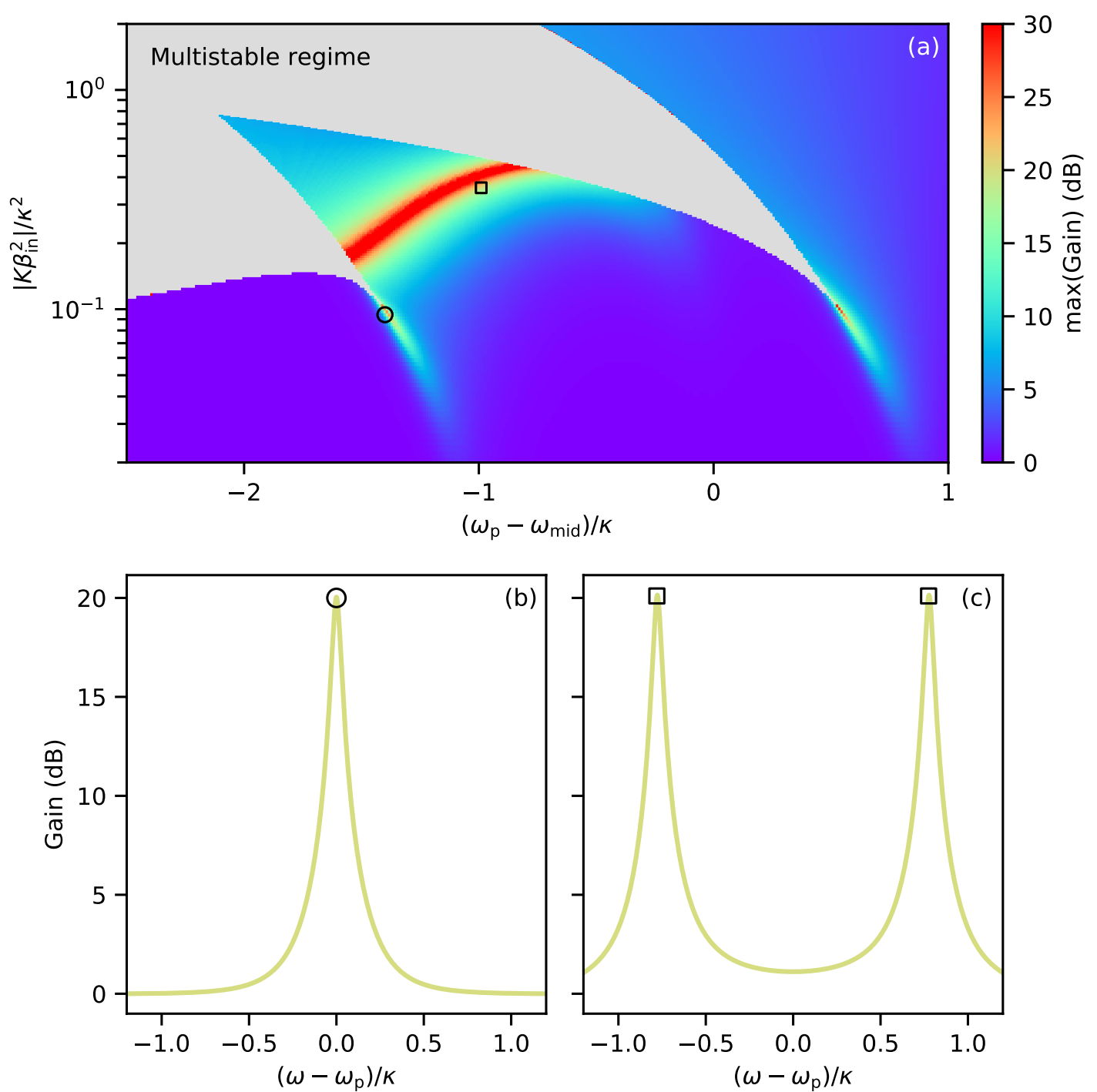

Figure 3.2: (a) Maximum of gain $G_{0}$ shown versus normalized pump power $\left|K \beta_{\text {in }}^{2}\right| / \kappa^{2}$ and frequency $\left(\omega_{\mathrm{p}}-\omega_{\text {mid }}\right) / \kappa$, where $\omega_{\text {mid }}=\left(\omega_{l}+\omega_{\mathrm{r}}\right) / 2$. While there are solutions with the maximum of gain reaching up to $60 \mathrm{~dB}, G_{0}$ was capped at $30 \mathrm{~dB}$ to make areas with degenerate gain more visible. The light-grey area indicates pump parameters for which Eq. (3.4) has multiple solutions. (b),(c) Examples of degenerate and non-degenerate gain profiles, respectively. The corresponding pump parameters are indicated by the corresponding circle and square labels in panel (a). 
In Fig. 3.2(a), maximum of power signal gain $G=|g|^{2}$ is show for different normalized pump powers $\left|K \beta_{\text {in }}^{2}\right| / \kappa^{2}$ and frequencies $\left(\omega_{\mathrm{p}}-\omega_{\text {mid }}\right) / \kappa$. The calculation was performed for the following system parameters: $\omega_{\mathrm{l}}=\omega_{\mathrm{r}}=2 \pi \cdot 6 \mathrm{GHz}, \kappa=J=2 \pi \cdot 600 \mathrm{MHz}, \gamma=0, K_{1}=K_{\mathrm{r}}=2 \pi \cdot 10 \mathrm{kHz}$. Depending on pump power and frequency, there are three different regions corresponding to different types of the amplifier response. The light-grey area in Fig. 3.2(a) indicates the region with multiple solutions of Eq. (3.4), resulting in bifurcation. If pump is applied in the vicinity of one of the hybridized modes, visible in Fig. 3.2(a) as "comets", only one of them is driven, resulting in the degenerate gain profile shown in Fig. 3.2(b). When the pump frequency is located in-between the modes, the system works as a non-degenerate amplifier (see Fig. 3.2(c)) with a two-lobe gain profile. The distance between Lorentzian lobes is approximately equal to $2 J$, ensuring signal-pump detuning (Sec. 2.3.3). Notably, in the non-degenerate regime the gain is tunable within $B_{\text {tun }} \sim \kappa$ thanks to the corresponding parameter region in Fig. 3.2(a). Thus, even without utilization of SQUIDs, JPD amplifiers are frequency-tunable within few hundreds of $\mathrm{MHz}$, on-par with some SQUID-based designs $\left[\mathrm{RFN}^{+} 12\right]$.

\subsubsection{Essential requirements for non-degenerate gain}

To ensure that the non-degenerate pump-parameter region is present, two conditions have to be fulfilled. First, it should be possible to pump both modes simultaneously. For that, modes splitting should be limited to $J \leq 2 \kappa$, as can be seen in Fig.3.3. As the ratio $J / \kappa$ increases, the effective area of pump parameters enabling non-degenerate gain decreases, almost disappearing for $J=3 \kappa$ (see Fig.3.3(c)).

Second, dimer modes need to be fully hybridized, meaning that $\kappa_{+}=\kappa_{-}$(or equivalently, $\omega_{1}=\omega_{\mathrm{r}}$ ). Asymmetry in the effective Bose-Hubbard modes leads to one modes having lower bifurcation photon number, resulting in reduction of the non-degenerate pump parameter region. The extreme case of $\omega_{\mathrm{r}}=$ $\omega_{1}+3 \kappa$ is shown in Fig. 3.4. As can be seen, for these parameters non-degenerate regime cannot be achieved. To avoid that, one should ensure that $\left|\omega_{\mathrm{r}}-\omega_{1}\right| / \kappa<1 / 2$.

\subsection{DJJAA concept}

To create a 4-wave-mixing non-degenerate amplifier with high saturation power, we utilized Josephson junction arrays (JJAs) with an engineered dispersion relation [WTR $\left.{ }^{+} 20\right]$. In this section, I discuss characteristics of a JJA, as well as its dispersion engineering.

\subsubsection{Josephson junction array (JJA)}

For a JJA-based amplifier design, it is crucial to be able to predict its dispersion relation and Kerr coefficients. Here, I focus on a particular case, crucial for amplifiers discussed in this thesis: a $\lambda / 2$ resonator made of an array of Josephson junctions. Its schematic is shown in Fig. 3.5(a). The array ends are galvanically connected to a $50 \Omega$ transmission line (left edge) and to the ground (right edge). The crossed boxes indicate Josephson junctions with critical current $I_{\mathrm{J}}$ and shunting capacitance $C_{\mathrm{J}}$. Superconducting strips connecting JJs create capacitance to ground $C_{0}$.

Following $\left[\mathrm{WKD}^{+} 15\right]$, this system can be described by the Lagrangian

$$
L=\sum_{i=1}^{N} \frac{C_{0}}{2} \dot{\Phi}_{i}^{2}+\sum_{i=0}^{N-1} \frac{C_{\mathrm{J}}}{2}\left(\dot{\Phi}_{i+1}-\dot{\Phi}_{i}\right)^{2}-\sum_{i=0}^{N-1} E_{J} \cos \left(\frac{\Phi_{i+1}-\Phi_{i}}{\varphi_{0}}\right)
$$



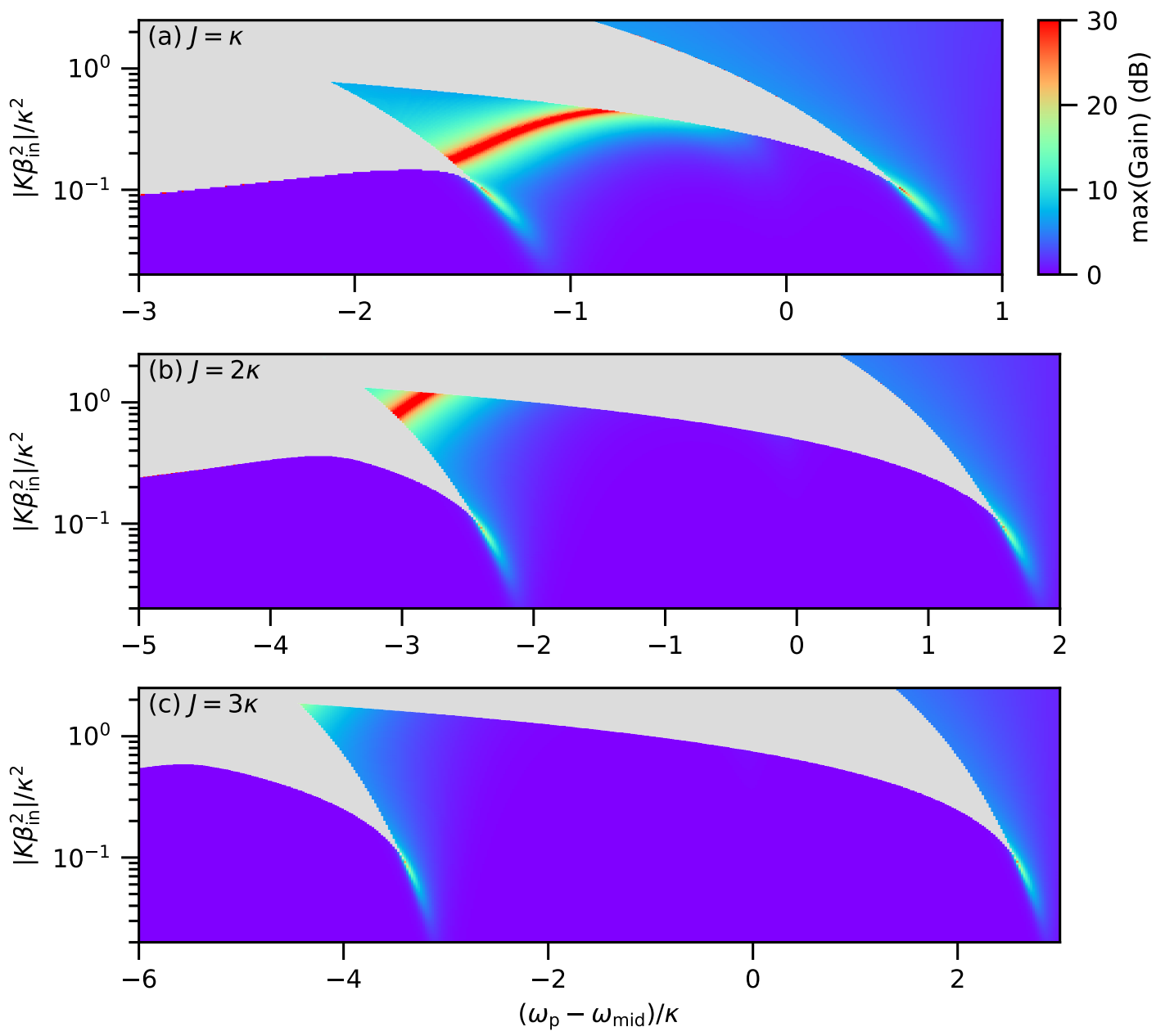

Figure 3.3: Maximum of gain shown versus normalized pump power $\left|K \beta_{\text {in }}^{2}\right| / \kappa^{2}$ and frequency $\left(\omega_{\mathrm{p}}-\omega_{\text {mid }}\right) / \kappa$ for different resonator coupling strengths: (a) $J=\kappa$, (b) $J=2 \kappa$, (c) $J=3 \kappa$. Light-grey areas indicate pump parameters for which Eq. (3.4) has multiple solutions. The calculation was performed for the following system parameters: $\omega_{\mathrm{l}}=\omega_{\mathrm{r}}=2 \pi \cdot 6 \mathrm{GHz}$, $\kappa=2 \pi \cdot 600 \mathrm{MHz}, \gamma=0, K_{1}=K_{\mathrm{r}}=2 \pi \cdot 10 \mathrm{kHz}$.

where $\Phi_{i}$ is the flux at node $i$, and $\Phi_{0}$ is the flux quantum. The dispersion of the JJA can be calculated by linearizing this Lagrangian. The linear part can be written in matrix form

$$
L_{\text {lin }}=\frac{1}{2} \dot{\Phi}^{\mathrm{T}} C \dot{\Phi}^{\mathrm{T}}-\frac{1}{2} \Phi^{\mathrm{T}} L^{-1} \Phi^{\mathrm{T}}
$$

with $\Phi=\left(\Phi_{0}, \Phi_{1}, \ldots, \Phi_{N}\right)^{\mathrm{T}}$. Here, $C$ and $L$ are the capacitance and inductance matrices (see Appendix A.1.1 for details). It can be quantized and numerically diagonalized, resulting in the Hamiltonian

$$
H_{\text {lin }}=\sum_{i=0}^{N} \hbar \omega_{i} a_{i}^{+} a_{i},
$$

where eigenfrequencies $\omega_{i}$ are calculated by solving the eigenvalue problem

$$
C^{-1 / 2} L^{-1} C^{-/ 2} \psi_{i}=\omega_{i}^{2} \psi_{i}
$$




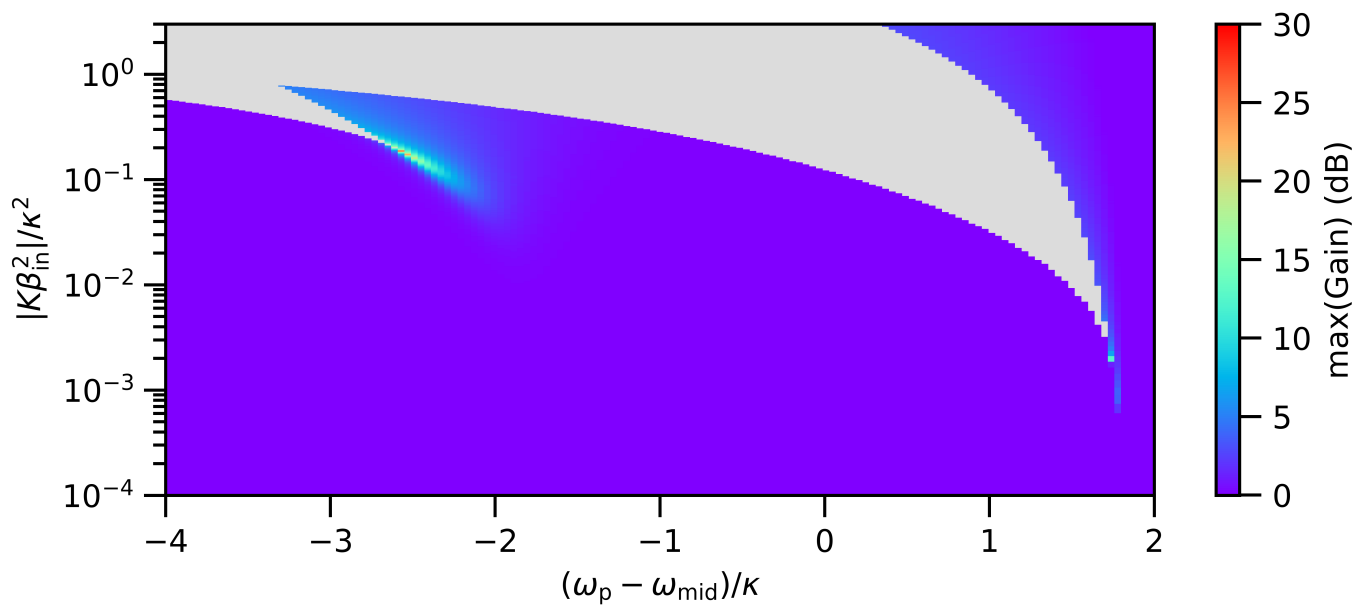

Figure 3.4: Maximum of gain shown versus normalized pump power $\left|K \beta_{\text {in }}^{2}\right| / \kappa^{2}$ and frequency $\left(\omega_{\mathrm{p}}-\omega_{\text {mid }}\right) / \kappa$ for the resonators with different resonance frequencies. Light-grey areas indicate pump parameters for which Eq. (3.4) has multiple solutions. Calculation was performed for the following system parameters: $\omega_{1}=2 \pi \cdot 6 \mathrm{GHz}, \kappa=2 \pi \cdot 600 \mathrm{MHz}, \gamma=0$, $\omega_{\mathrm{r}}=\omega_{\mathrm{l}}+3 \kappa, J=\kappa, K_{\mathrm{l}}=K_{\mathrm{r}}=2 \pi \cdot 10 \mathrm{kHz}$.

Here, eigenvectors $\psi_{i}$ are linked to the flux spatial distribution of the corresponding modes as

$$
\Phi_{i}=C^{-1 / 2} \psi_{i} \sqrt{\frac{\hbar}{2 \omega_{i}}}\left(a_{i}+a_{i}^{+}\right)
$$

A typical solution of the eigenproblem is shown in Fig. 3.5(b), for chain parameters listed in Table 3.1. The modes dispersion is linear for $\omega_{i} \ll \omega_{\text {plasma }}$, saturating to the plasma frequency $\omega_{\text {plasma }}=1 / \sqrt{L_{\mathrm{J}} C_{\mathrm{J}}}$ as mode number increases. Flux distribution for the linear part of the dispersion is sinusoidal (see Fig. 3.5(c)), similar to microstrip resonator modes [Poz11].

To calculate self- and cross-Kerr coefficients of the JJA, the Lagrangian in Eq. (3.9) is expanded to the forth order $\left[\mathrm{WKD}^{+} 15\right]$ :

$$
H=\sum_{j} \hbar w_{j} a_{j}^{+} a_{j}+\frac{\hbar}{2} \sum_{j} K_{j j} a_{j}^{+} a_{j}^{+} a_{j} a_{j}+\sum_{\{j, k \mid j \neq k\}} K_{j k} a_{j}^{+} a_{j} a_{k}^{+} a_{k}
$$

By using perturbation theory and utilizing the solution obtained for the linearized Lagrangian, the Kerr coefficients can be written as

$$
\begin{gathered}
K_{j j}=-12 \eta_{j j j j} \frac{\hbar E_{J}}{\varphi_{0} C_{\mathrm{J}}^{2} w_{j}^{2}} \\
K_{j k}=-12 \eta_{j j k k} \frac{\hbar E_{J}}{\varphi_{0} C_{\mathrm{J}}^{2} w_{j} w_{k}}
\end{gathered}
$$

Here, $\eta_{i j k l}$ is a numerical coefficient, exact formula of which can be found in [WKD $\left.{ }^{+} 15\right]$. 


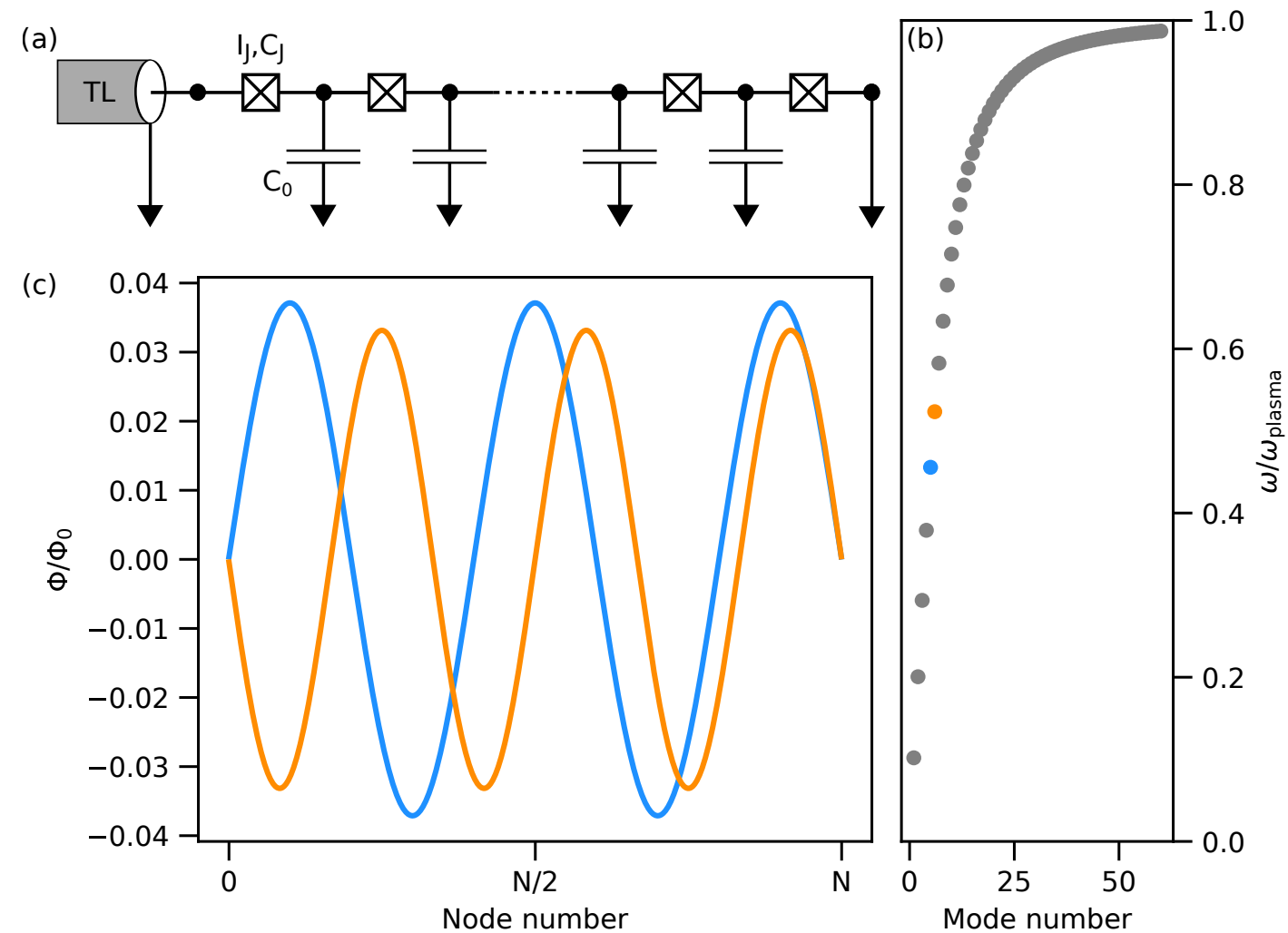

Figure 3.5: (a) Schematic of a Josephson junction array-based $\lambda / 2$ resonator. (b) Mode dispersion of the JJA calculated for parameters listed in Table 3.1. (c) Distribution of the flux over the resonator for two neighbouring modes indicated in panel (b), i.e. the eigenstates of modes 5 and 6.

\subsubsection{Dimerization of a JJA}

To ensure the $J \leq 2 \kappa$ condition (see Sec. 3.1.1), the JJA dispersion relation can be engineered to achieve the required modes splitting. In the scope of this thesis, it was done by introducing a series capacitor in the middle of the array shown in Fig. 3.6(a). This results in symmetric and asymmetric modes (Fig. 3.6(c)). These modes form pairs, denoted dimers, as can be seen in Fig. 3.6(b). The frequency splitting between modes of a dimer is controlled by the value of $C_{c}$ (see Fig. 3.7), providing a convenient tuning knob for amplifier design. Each dimer located below the plasma frequency region can be utilized for non-degenerate 4-wave-mixing amplification.

Table 3.1: List of parameters used for JJA/DJJAA modes simulation.

\begin{tabular}{|c|c|c|c|c|c|c|c|}
\hline Type & $\mathrm{N}$ & $I_{\mathrm{J}}$ & $C_{\mathrm{J}}$ & $\omega_{\text {plasma }} / 2 \pi$ & $C_{0}$ & $C_{\mathrm{c}}$ & $C_{0}^{\prime}$ \\
\hline JJA (Fig. 3.5(a)) & 1600 & $3 \mu \mathrm{A}$ & $1225 \mathrm{fF}$ & $13.72 \mathrm{GHz}$ & $0.45 \mathrm{fF}$ & N/A & N/A \\
\hline DJJAA (Fig. 3.6(a)) & 1600 & $3 \mu \mathrm{A}$ & $1225 \mathrm{fF}$ & $13.72 \mathrm{GHz}$ & $0.45 \mathrm{fF}$ & $40 \mathrm{fF}$ & $33 \mathrm{fF}$ \\
\hline
\end{tabular}


(a)

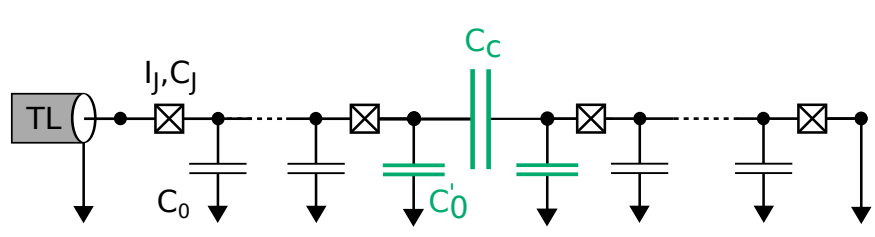

(c)
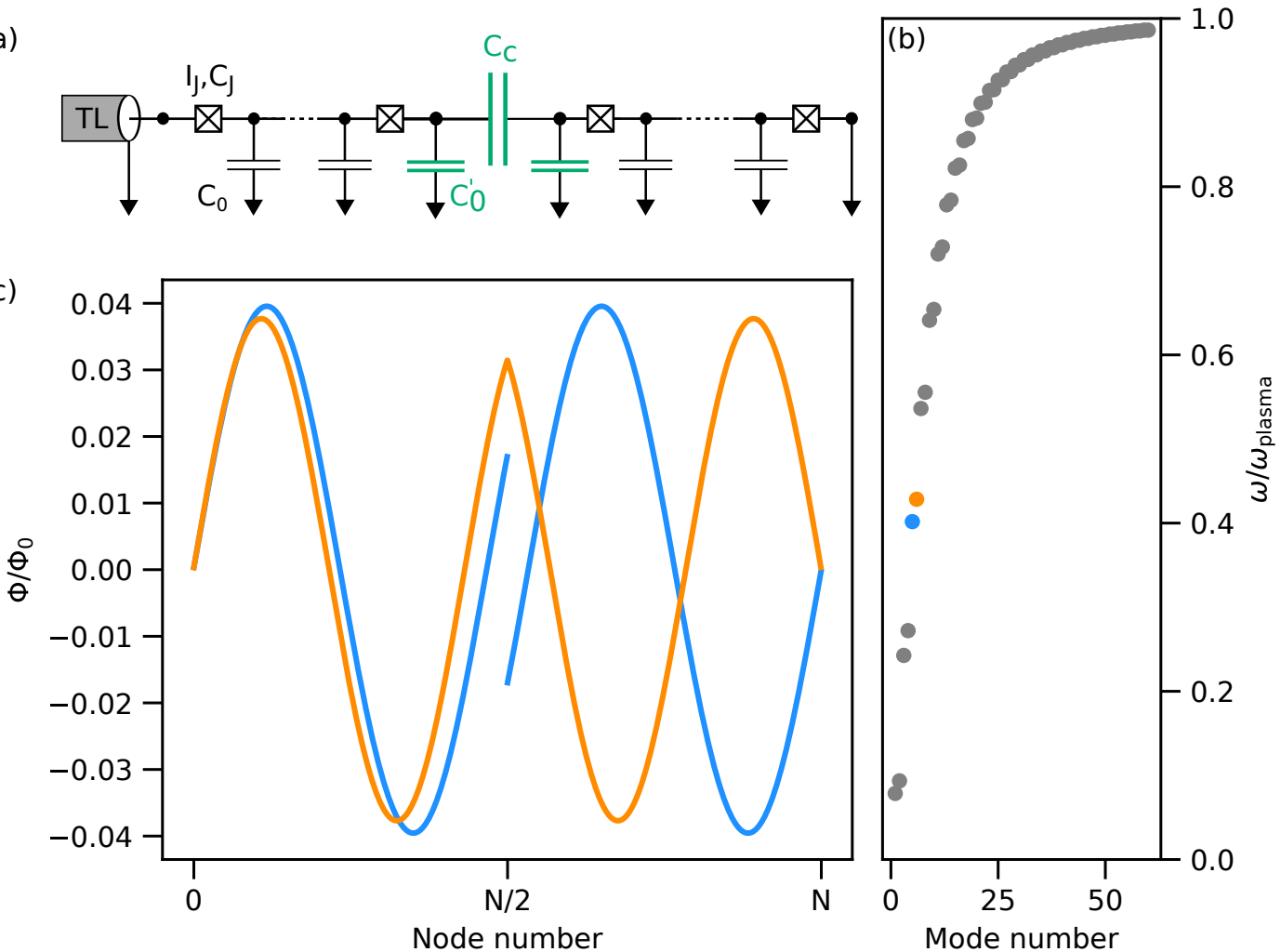

Figure 3.6: (a) Schematic of a dimerized Josephson junction array. (b) Modes dispersion of the DJJA calculated for parameters listed in Table 3.1. (c) Distribution of a flux over the resonator for two neighbouring modes indicated in panel (b).

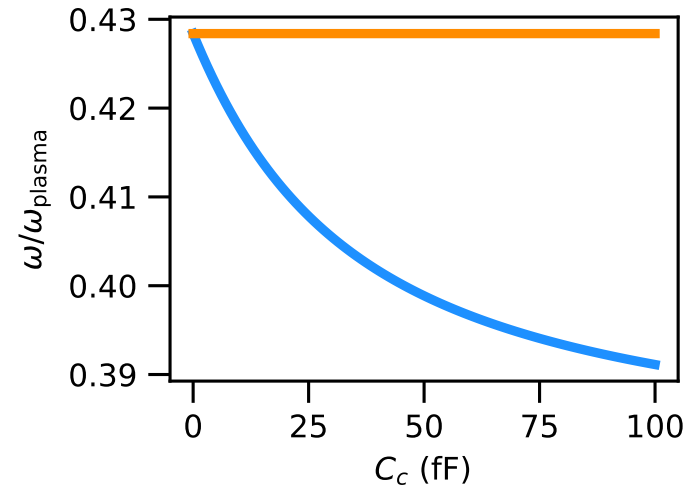

Figure 3.7: Frequencies of selected modes as a function of $C_{c}$. Colors indicating different modes corespond to the ones used in Fig. 3.6(b). Notice that only half of the modes (every second one) are influenced by the coupling capacitor. This can be intuitively understood as a consequence of the eigenstates shown in Fig. 3.5(c), which indicate that only half of the modes have a voltage gradient at the position of the capacitor.

\subsection{Experimental realization}

Here, I present a DJJAA [WTR $\left.{ }^{+} 20\right]$ fabricated with a two-step optical lithography. The amplifier shown in Fig. 3.8 was further used for high-power detection of a grAl fluxonium quantum jumps discussed in Chapter 4. For information about other fabricated and measured optical-lithography DJJAAs, the reader is 
(a)

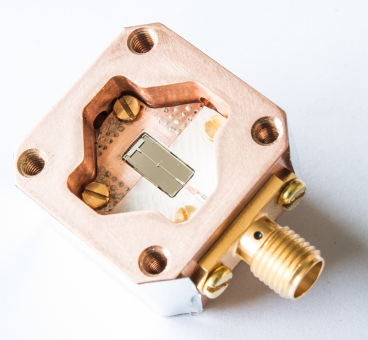

(b)

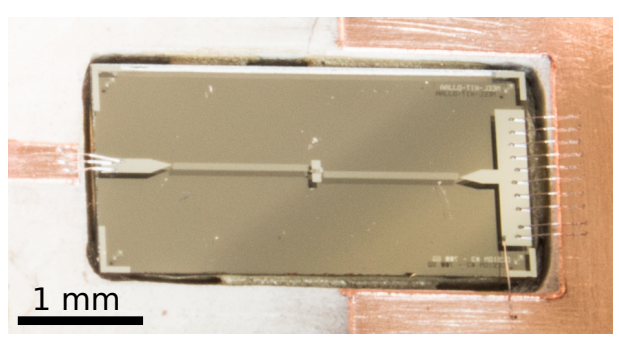

(c)

(d)

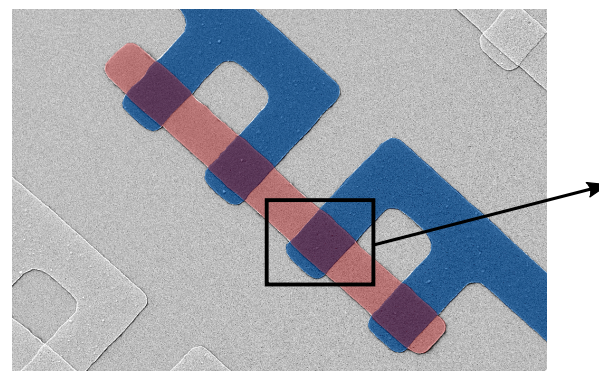

(e)

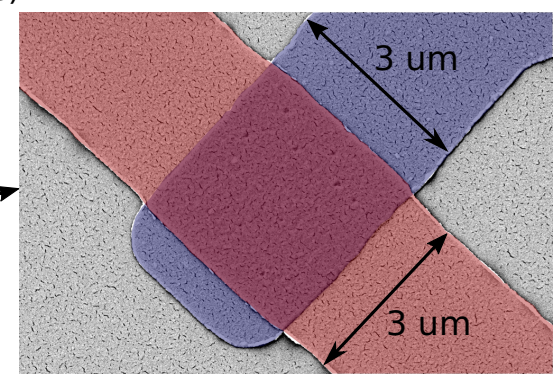

Figure 3.8: (a),(b) Photo of a DJJAA inside a sampleholder. (c) SEM images of SQUID array and interdigitated capacitor. (d),(e) Falsecolored SEM images of a SQUID. Blue color corresponds to the first Al layer, blue - to the second one.

referred to [Win20]. A DJJAA fabricated by using e-beam lithography relying on a bridge-free technique [LPP $\left.{ }^{+} 11\right]$, similar to Ref. [PDM ${ }^{+}$19], is discussed in Appendix A.1.2.

In Fig. 3.8(c), SEM images of the DJJAA are presented. The amplifier consists of an array of 1600 SQUIDs interrupted in the middle with an interdigitated capacitor. Left and right edges of the array are galvanically connected to a $50 \Omega$ microstrip transmission line and ground plane, respectively. Circuit parameters of the amplifier correspond to the ones listed in Table 3.1 for the DJJAA.

The sample was fabricated on top of a polished $330 \mu \mathrm{m}$ thick C-plane sapphire wafer by utilizing a twostep optical lithography [WTR ${ }^{+}$20]. First aluminum layer (30 nm thick) including capacitor pads, on-chip microstrip lines, and part of the array, is shown in the magnified images of the SQUID area (Figs. 3.8(d,e)) in a blue false color. Josephson junctions were formed by oxidation of the first layer to form the oxide layer followed by evaporation of the second layer of aluminum ( $40 \mathrm{~nm}$ thick) shown in red in Figs. 3.8(d,e). Prior to the latter two steps, an argon milling was performed $\left[\mathrm{GMS}^{+}{ }^{18]}\right.$ to remove native oxide forming due to 
exposing the sample to atmosphere. Before each $\mathrm{Al}$ evaporation, plasma cleaning was done to prevent aging of Josephson junctions $\left[\mathrm{PFC}^{+} 12\right]$. To ensure uniformity of the ground capacitances and to minimize losses, a $200 \mathrm{~nm}$ thick platinum layer was deposited on the backside of the chip. The sample was then glued to the copper sampleholder with a silver paste (Fig. 3.8(a)) and connected to a printed circuit board by aluminum wirebonds (Fig. 3.8(b)).

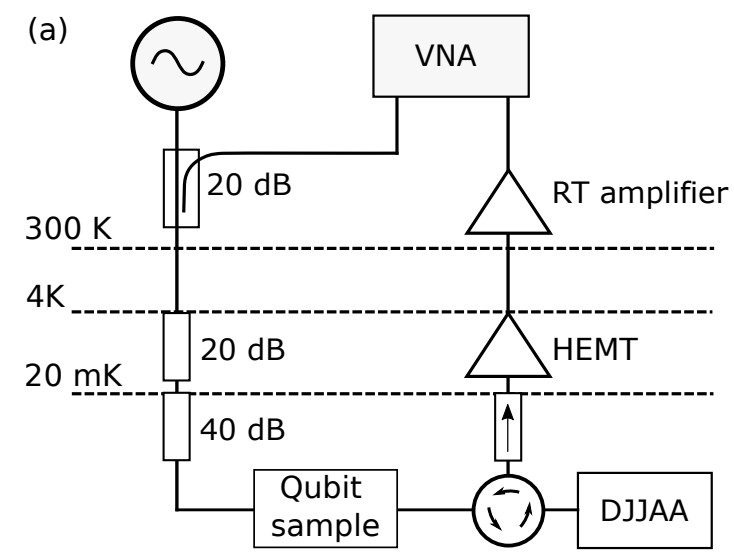

(c)

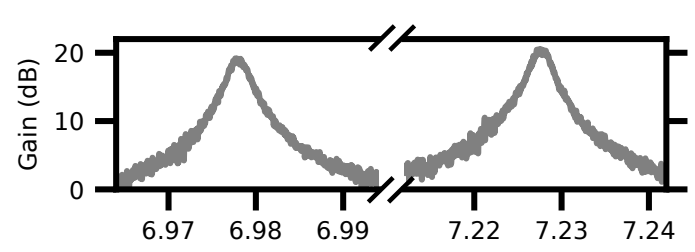

(d)

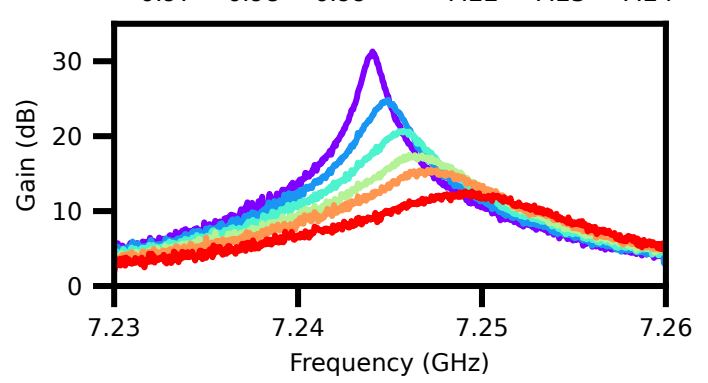

(b)

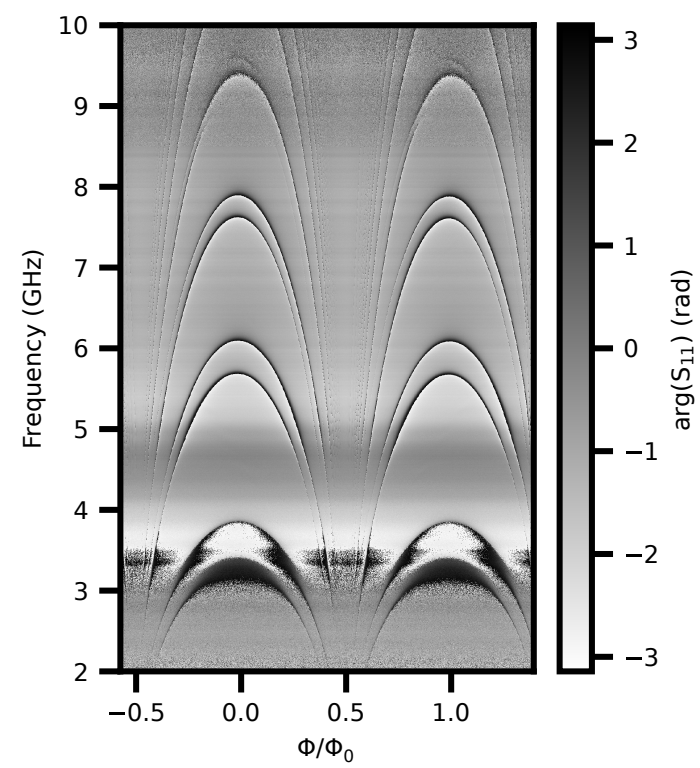

(e)

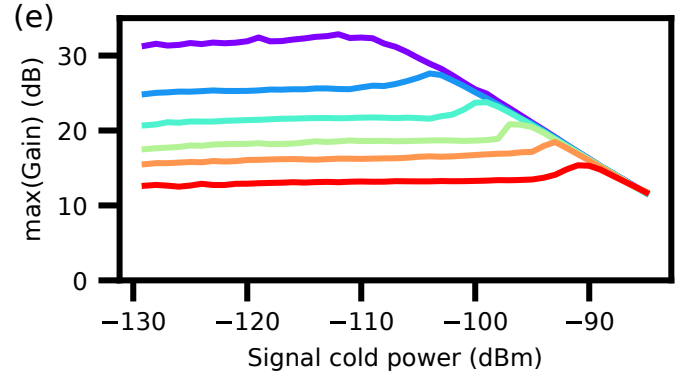

Figure 3.9: (a) Schematic of experimental setup used for DJJAA characterization. The displayed microwave components are thermalized to the nearest temperature stage indicated above them. (b) Phase of weak reflected signal versus frequency and external magnetic flux. (c) Example of a non-degenerate gain profile measured at $\Phi \approx 0.2 \Phi_{0}$ (d) Left lobe of non-degenerate gain versus signal frequency measured for different pump powers at $\Phi \approx 0.09 \Phi_{0}$. (e) Maximum of gain profiles shown in panel (d) versus input signal power.

To characterize the DJJAA, we used the experimental setup shown in Fig. 3.9(a). The qubit sample in front of the DJJAA is used for power calibration which is discussed in details in Sec. 3.4. Fig. 3.9(b) shows the phase of the reflection coefficient of a weak probe measured for different external magnetic fields. There are two dimers which can be used for non-degenerate amplification in the 4-8 GHz octave. In this section, I focus on the upper one. In Fig. 3.9(c), and example of a non-degenerate gain obtained at $\Phi / \Phi_{0} \approx 0.2$ is presented.

For the grAl fluxonium state readout (Chapter 4), the amplifier was flux-tuned to $\Phi / \Phi_{0} \approx 0.1$ to match the readout resonator frequency. At this external flux, the linear parameters of the DJJAA extracted with a circle fit $\left[\mathrm{PSB}^{+} 15\right]$ are: $w_{-} / 2 \pi=7.055 \mathrm{GHz}, w_{+} / 2 \pi=7.279 \mathrm{GHz}, \kappa_{-} / 2 \pi=54 \mathrm{MHz}, \kappa_{+} / 2 \pi=88 \mathrm{MHz}$, $\gamma / \kappa \leq 0.1$. Fig. 3.9(d) shows left lobes of non-degenerate gain profiles with the lobes splitting of about $260 \pm 20 \mathrm{MHz}$ measured at the external flux $\left(\Phi / \Phi_{0} \approx 0.1\right)$. For these gains, pump frequency was kept 
constant $\left(\omega_{\mathrm{p}} / 2 \pi=7.377 \mathrm{GHz}\right)$ while pump power was varied. For the $20 \mathrm{~dB}$ gain profile, the instantaneous bandwidth is equal to $7 \mathrm{MHz}$. In Fig. 3.9(e), maximum of gain is shown as a function of calibrated signal power at the input of the DJJAA. This dependence allows to extract the saturation power, defined as signal power for which maximum of gain reduces by $1 \mathrm{~dB}$. For the $20 \mathrm{~dB}$ gain, the saturation power was $-98 \mathrm{dBm}$, equaivalent to about $2 \cdot 10^{4}$ photons/ $\mathrm{us}$.

\subsection{Power calibration}

In situ calibration of power at the input of the device of interest is important in cQED. It is instrumental for a number of routines, including (but not limited to) measuring saturation power of an amplifier, determining Kerr coefficient of a nonlinear system, and measuring the noise added by an amplifier. In this section, I present an experimental realization of power calibration relying on scattering effects discussed in Sec. 2.2.1. In particular, a transmon qubit directly coupled to a transmission line (Sec. 3.4.1) and a qubit-resonator system (Sec. 3.4.2) made of a granular aluminum fluxonium qubit coupled to a readout antenna [GSG ${ }^{+} 19$; $\left.\mathrm{GSG}^{+} 21\right]$ were utilized. Another power calibration technique based on an ac-Stark effect is discussed in Sec. 4.4.

\subsubsection{Scattering from a transmon qubit coupled to a transmission line}

(a)
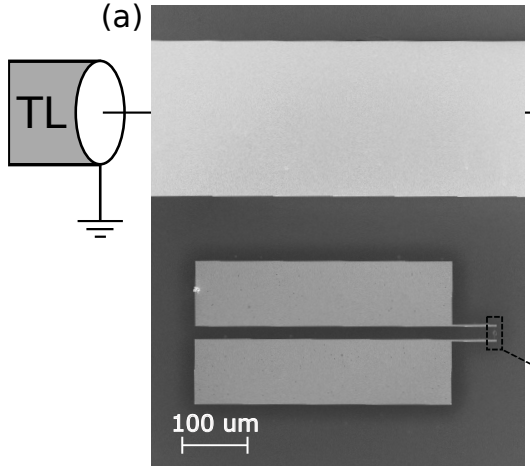

(b)

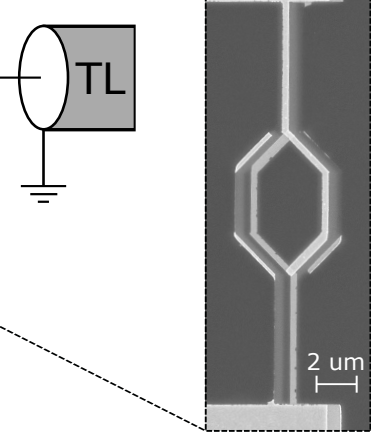

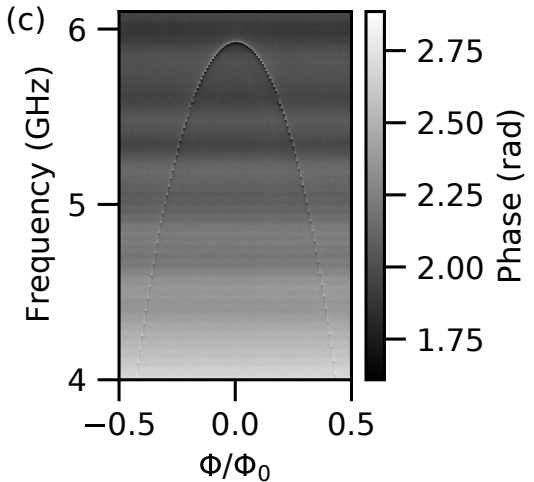

Figure 3.10: (a) SEM image of the transmon qubit (the bottom object) capacitively coupled to a transmisson line (the top object). The qubit is formed by capacitor pads connected by a SQUID shown in panel (b). (c) Spectrum of the transmon qubit: phase of weak probe versus frequency and external magnetic flux.


Figure 3.11: (a) Transmission at the probe frequency $\omega=\omega_{01}$, where $\omega_{01}$ is the transmon qubit, shown as a function of calibrated probe power. The upper x axis shows probe power in units of photons in the band $N_{\text {photons }} / \gamma$, where $N_{\text {photons }}=P_{\text {probe }} / \hbar \omega_{01}$ is the photon flux and $\gamma$ is the qubit $T_{2}$ rate. (b) IQ parametric plots of transmission for different probe powers with probe frequency being the parameter. Lines' colors are in agreement with panel (a). 
To calibrate the saturation power of the DJJAA device described in Appendix A.1.2, we utilized a transmon qubit capacitively coupled to a microstrip transmission line. In Fig. 3.10(a), an SEM image of the qubit is shown. It consists of capacitor pads connected by a dc-SQUID, magnified image of which is presented in Fig. 3.10(b). The SQUID is formed of Al superconducting loop and two Al/AlOx/Al Josephson junctions fabricated on top of a C-plane sapphire substrate.

In the absence of magnetic field, transmon parameters are: $\omega_{01} / 2 \pi=\frac{1}{h}\left(\sqrt{8 E_{\mathrm{J}} E_{\mathrm{C}}}-E_{\mathrm{C}}\right)=5.953 \mathrm{GHz}$, $E_{\mathrm{J}}=16.31 \mathrm{GHz}, E_{\mathrm{C}}=300 \mathrm{MHz}$. The Josephson energy (and thus qubit frequency) can be tuned by applying external magnetic field. Fig. 3.10(b) shows the spectrum of of the qubit. Owing to is tunability, the transmon can be used as a power calibration unit in $4-6 \mathrm{GHz}$ frequency range. Moreover, after using it to calibrate power at the frequency of interest, the qubit can be flux-tuned below $4 \mathrm{GHz}$ to avoid its influence on further experiments.

As was discussed in Sec. 2.2.1, two effects can be used for a power calibration: resonance fluorescence and Autler-Mollow triplet. In case of resonanse fluoresncence, also referred to as elastic scattering, transmission through the microstrip $50 \Omega$ line is power-dependent. The transfer coefficient obeying Eq. (2.18), aside from $\omega_{01}, E_{\mathrm{J}}, E_{\mathrm{C}}$, depends on drive power $P_{\text {probe }}$, coupling strength to the transmission line $\kappa / 2 \pi=$ $3.24 \mathrm{MHz}$, and decoherence rate $\gamma=1.78 \mathrm{MHz}$. The latter two were extracted from a circle fit [PSB $\left.{ }^{+} 15\right]$ applied to low-power $(\Omega \ll \kappa)$ transmission data. With the decay rates being known, transmission $S_{21}(\omega)$ depends only on drive power, and the exact value of the latter can thus be extracted from transmission measurements. Fig. 3.11(a) shows the measured transmission in zero magnetic field at $\omega=\omega_{01}$. The black solid line indicates a fit with Eq. (2.18), yielding attenuation between VNA (Fig.3.9(a)) and the qubit $A=P_{\text {probe }} / P_{\mathrm{VNA}}=92.4 \pm 0.1$. In Fig. 3.11(b), the transmission function plotted in IQ plane is compared with the fit result for different probe powers.



Figure 3.12: Transmission of a weak probe shown as a function of calibrated power of the dressing microwave drive.

Another calibration approach, denoted inelastic scattering, relies on the Autler-Townes effect. If a strong drive $(\Omega \gg \kappa)$ is applied, states of qubit and propagating photons get dressed. When probing such a dressed system with a weak probe, three modes are visible, with drive-power-dependent frequencies equal to $\omega_{01}, \omega_{01} \pm \Omega$. Fig. 3.12 demonstrates the inelastic scattering measured with VNA (Fig.3.9(a)) for $\Phi=0$. Three modes indicated with dotted colored lines are the Mollow triplet modes, appearing due to a strong drive created by a microwave generator. The other two frequencies located at lower frequencies are present due to the 1-2 transition of the transmon. By fitting the Mollow triplet frequencies' power dependence (see Eq. (2.22)), the attenuation between microwave generator and the transmon qubit $A=P_{\text {cold }} / P_{\text {gen }}=95.5 \pm 0.1$ was extracted. It differs from the one obtained by the elastic scattering method due to different cabling of VNA and microwave generator. 


\subsubsection{Scattering from a qubit-resonator system}

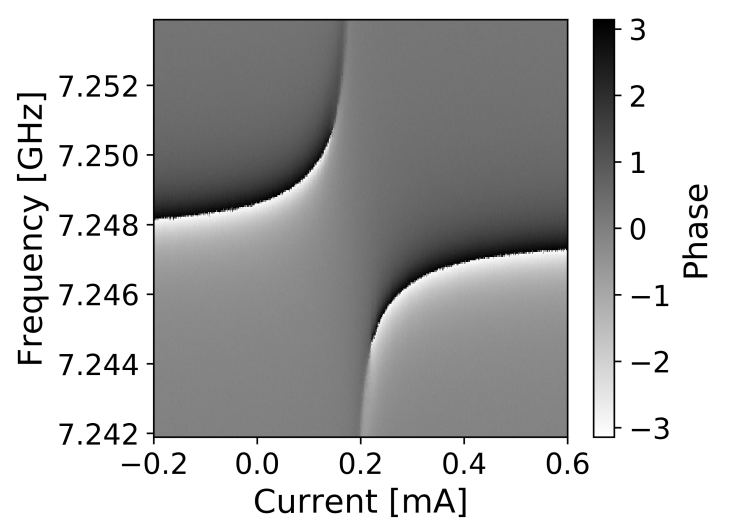

Figure 3.13: Phase of a probe signal versus current through a coil creating magnetic field in the fluxonium loop (cf. Fig. 2.2).


Figure 3.14: (a) Reflection at the probe frequency corresponding to frequency of the hybridized mode shown as a function of calibrated probe power. The upper $\mathrm{x}$ axis shows probe power in units of photons in the band $N_{\text {photons }} / \gamma$, where $N_{\text {photons }}=P_{\text {probe }} / \hbar \omega_{01}$ is the photon flux and $\gamma$ is the mode's $T_{2}$ rate. (b) IQ parametric plots of transmission for different probe powers with probe frequency being the parameter. Lines' color is in agreement with panel (a).

Scattering effects can be utilized for power calibration not only for a qubit directly coupled to a transmission line, but also for a standard dispersive readout setup in which the qubit is coupled to a readout resonator with a coupling strength $g$, and the resonator is coupled to a transmission line. By flux-tuning the qubit frequency to the vicinity of the resonator frequency, such that $\left|\omega_{01}-\omega_{\text {res }}\right| \ll g$, one creates a system of two strongly hybridized qubit-resonator modes. Each of them can be thought of and used as a two-level-system directly coupled to a transmission line.

In Fig. 3.13, a spectrum of a strongly hybridized qubit-resonator system is shown. The measured system (for details, see Chapter 4) is a granular aluminum fluxonium qubit coupled to a readout antenna. Fig. 3.14 presents an example of the elastic scattering applied to the lower mode at the coil current $I=1.768 \mathrm{~mA}$. The effective system parameters extracted following the procedure discussed in the previous section are: $\omega_{01}=7.226 \mathrm{GHz}, \kappa=5.4 \mathrm{MHz}, \gamma=3.4 \mathrm{MHz}$. The elastic scattering was utilized to measure the saturation power of the DJJAA discussed in this chapter. The result of this calibration yeilding $P_{\text {sat }}=-98 \pm 1 \mathrm{dBm}$ is in agreement with the ac-Stark power photon number calibration (see Sec. 4.4). 



\section{$4 \quad$ High-power quantum jumps detection}

In this section I demonstrate speed-up in detection of quantum jumps of a superconducting artificial atom achieved by utilizing readout signal power up to two orders of magnitude higher than the state-of-the-art. This was made possible by the unique combination of a DJJAA, and the fact that the readout of our granular aluminum fluxonium artificial atom remained quantum-non-demolition (QND) at relatively large photon numbers in the readout resonator, up to $\bar{n}=110$. Using Bayesian inference allows to detect quantum jumps faster than the readout resonator response time.

\subsection{Measurement strength}
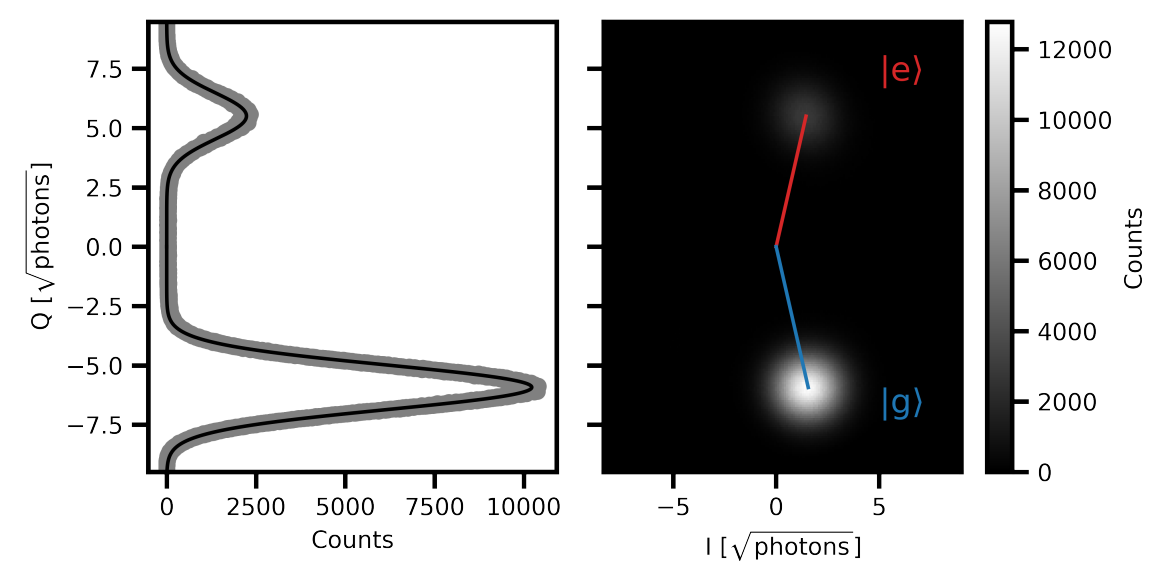

Figure 4.1: Histograms of the measured continious readout response: Q quadrature (left panel) fitted with gaussian functions and the IQ response(right panel). Colored lines on the right panel indicate mean value of the steady-state complex amplitude.

For measurement of a state of an artificial atom dispersively coupled to a readout resonator one of the most important things to consider is if the measurement is weak or a strong projective. The measurement strength is determined by the SNR, defining whether a single measured data point is sufficient (projective measurement) or insufficient (weak measurement) to infer the state of the monitored quantum object. For a two-level system, SNR is defined as the ratio of distance between steady states $\alpha_{\mathrm{g} / \mathrm{e}}$ corresponding to qubit states and the sum of their variance $\sigma_{\mathrm{g} / \mathrm{e}}$

$$
\mathrm{SNR}=\frac{\left|\alpha_{\mathrm{e}}-\alpha_{\mathrm{e}}\right|}{\sigma_{\mathrm{e}}+\sigma_{\mathrm{e}}},
$$

both inferred from a Gaussian fit of a histogramed sequence of consecutive measurements. A typical example of such a histogram is shown in Fig. 4.1. The SNR is affected not only by the obvious parameters, such as readout power and system noise, but also by some other readout-line related parameters, which will be addressed further in this section. 
Lets start with calculations of the expected SNR for the ideal (lossless) case. Without loss of generality, we can focus on a so-called steady-state SNR

$$
\mathrm{SNR}_{\mathrm{ss}}^{\text {ideal }}=\frac{|\alpha|}{\sqrt{\sigma_{Q}^{2}+\sigma_{I}^{2}}}=\sqrt{\frac{P_{\text {out }}}{P_{\text {noise }}}} .
$$

Here $\alpha$ is the pointer state, $\sigma_{I / Q}^{2}$ are it's variances along respective quadratures, $P_{\text {out } n \text { noise }}$ are the power of the readout signal and noise, respectively. $\mathrm{SNR}_{\mathrm{ss}}^{\text {ideal }}$ provides information about the readout chain, while the definition given in Eq. (4.1) combines this information with an angle between different steady states. The angle information is not a critical resource of the measurement strength since reaching a target coupling strength is not a problem in $\mathrm{CQED}$, and one can design qubit-resonator system with up to $180^{\circ}$ angle between the steady states resulting in $\mathrm{SNR}_{\mathrm{sS}} / \mathrm{SNR} \sim 1$.

For the readout resonator with a photon number population $\bar{n}$, resonance frequency $\omega_{\text {res }}$, and coupling strength $\kappa$, provided $Q_{c} \ll Q_{i}$, the readout drive power at the output is

$$
P_{\text {out }}=\frac{1}{4} \hbar \omega_{\text {res }} \bar{n} \kappa
$$

and the noise power is

$$
P_{\text {noise }}=k_{B} T_{\text {noise }} B \text {. }
$$

There are two discussion-worthy terms in the last equation, with the first one being the noise temperature $T_{\text {noise. }}$ It has two contributions: the natural noise $T_{\text {in }}$ coming with the signal (think of the noise at the output of the readout resonator), and the noise added by the readout chain $T_{\text {sys }}$. In case there is no squeezing involved (see Sec. 4.6) and the experimental setup has proper attenuation and thermalization [KSK ${ }^{+}$19], $T_{\text {in }}$ is at a quantum limit $T_{\text {in }}=\frac{\hbar \omega_{\text {res }}}{2 k_{B}}$. The second part, $T_{\text {sys }}$, is dominated by the added noise of the first amplifier in the chain (Sec. 2.3.3). Thus, for an ideal standard-quantum-limited setup utilizing a Josephson parametric amplifier (Sec. 2.3), $T_{\mathrm{sys}}=\frac{\hbar \omega}{2 k_{B}}$.

The other important factor for calculating the noise power is the measurement bandwidth $B$ (see supplementary material in Ref. [VSS11]). It is affected by all filtering bandwidths $B^{-1}=\sum_{i} B_{i}^{-1}$, including the effective filter given by the measurement time $B_{\text {meas }}=1 / \tau$ (the Fourier transformation of a rectangle pulse), readout resonator bandwidth $B_{\mathrm{rr}}=\kappa / 2$, bandwidth of the first amplifier in the amplification chain $B_{\text {amp }}$ and all other filters (e.g. bandpass ones at room temperature) present in the readout chain. It is thus important to make sure that the readout resonator bandwidth is much smaller than the bandwidth of JPA and all band-pass filters in order to avoid limiting the information flux. Provided that only $B_{\text {meas }}$ and $B_{\mathrm{rr}}$ are non-negligible, the ideal SNR can be calculated as

$$
\mathrm{SNR}_{\mathrm{ss}}^{\text {ideal }}=\frac{|\alpha|}{\sqrt{\sigma_{Q}^{2}+\sigma_{I}^{2}}}=\sqrt{\frac{\hbar \omega_{\text {res }} \bar{n} \kappa}{4 k_{B} T_{\text {noise }} B}}=\sqrt{\frac{1}{4} \bar{n} \kappa B^{-1}} \approx \sqrt{\frac{1}{4} \bar{n}(\kappa \tau+2)}
$$

To address real-setup imperfections in Eq. (4.5), so-called measurement efficiency $\eta$ is added to the equation.

$$
\mathrm{SNR}_{\mathrm{ss}}=\sqrt{\frac{1}{4} \eta \bar{n}(\kappa \tau+2)}
$$

In the state-of-the-art quantum-limited cQED setups, typical values of measurement efficiency are $\eta=0.4-$ $0.8\left[\mathrm{EKT}^{+} 19\right]$. Sources of non-ideality in the readout chain are cables, connectors and circulators/isolators 
losses between qubit and JPA, as well as the noise of the amplifier being higher than the expected quantum limit.

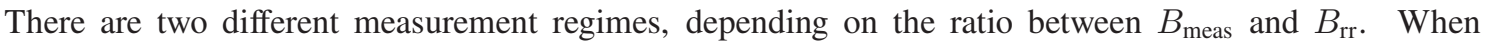
$B_{\text {meas }} \ll B_{\text {rr }}$ or, in other terms, $\kappa \tau \gg 1, \mathrm{SNR}_{\mathrm{ss}} \approx \sqrt{\frac{1}{4} \bar{n} \kappa \tau}$. This means that the measurement time is much bigger than the resonator response time $\tau_{B}=2 / \kappa$ (also commonly referred to as ring-up/down time), and after a measurement the data point is guaranteed to be in a $3 \sigma$ area of one of the steady states in Fig. 4.1. For this regime, SNR defined in Eq. (4.1) serves as a measure of the measurement strength. Provided big enough SNR, this results in a strong projective measurement, and later on in the text I will refer to this regime as 'large- $\tau$ ' regime.

A different situation occurs when $B_{\text {meas }} \gg B_{\mathrm{rr}}(\kappa \tau \ll 1)$. In that case the measurement time is less than the resonator response time, and $\mathrm{SNR}_{\mathrm{ss}} \approx \sqrt{\bar{n} / 2}$ depends only on the drive power due to saturation occurring when $\tau \rightarrow 0$. Although it seems that information gets lost in that regime, it is not the case, and a projective measurement regime can also be achieved. Moreover, as will be discussed in this chapter, this regime is actually the holy grail of the qubit state detection. However, the definition of SNR given in Eq. (4.5) is not applicable for that situation since the information about the qubit state is hidden behind the readout resonator dynamics. Thus the conventional SNR cannot be used as a measure of the measurement strength in this 'dynamical' regime, which requires a dedicated treatment discussed in Sec. 4.3.

\subsection{Decreasing the state discrimination time}

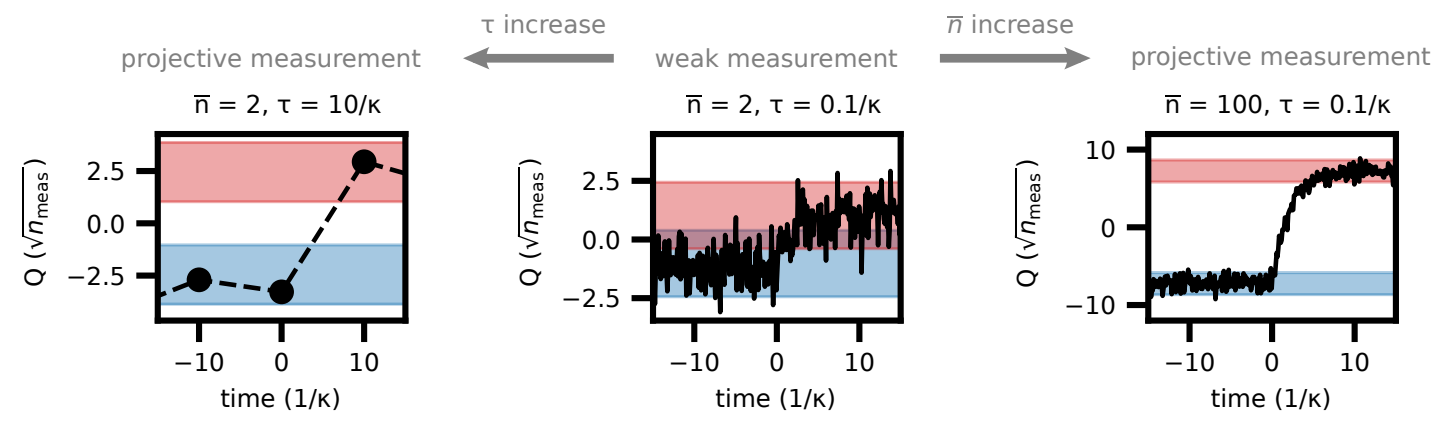

Figure 4.2: Simulated Q quadrature (black lines) of a quantum-limited continuous measurement of a qubit coupled to a readout resonator. The quadrature values are presented in units of square root of measurement photons $\sqrt{n_{\text {meas }}}=\sqrt{\bar{n} B^{-1} \kappa / 4}$, where $\bar{n}$ is the circulating photon number in the readout resonator, $\kappa$ is the resonator's coupling rate to the measurement apparatus, and $B^{-1} \approx \tau+\tau_{B}$ is the measurement bandwidth, given by the sum of the integration time $\tau$ and the resonator response time $\tau_{B}=2 / \kappa$. The blue and red areas indicate $\pm 2 \sigma$ intervals centered around the mean values of the $\mathrm{Q}$ quadrature corresponding to the ground and first excited state, respectively, denoted $Q_{|\mathrm{g}\rangle}$ and $Q_{|\mathrm{e}\rangle}$. The panels depict the transition from a weak measurement (middle panel) to a projective measurement by increasing either $\tau$ (left panel) or $\bar{n}$ (right panel).

During the continuous measurement of a qubit coupled to a readout resonator, schematically shown in Fig. 4.2, when the qubit state changes between ground $(|\mathrm{g}\rangle)$ and excited $(|\mathrm{e}\rangle)$, due to thermal or nonequilibrium (eg. quasiparticles [VPS $\left.{ }^{+} 14\right]$ ) excitations, the response of the readout resonator moves along the classical trajectory between the steady states corresponding to the $|\mathrm{g}\rangle$ and $|\mathrm{e}\rangle$ states of the qubit. The evolution of the measured Q quadrature during that transition is $Q(t)-Q_{|\mathrm{e}\rangle} \propto\left(Q_{|\mathrm{g}\rangle}-Q_{|\mathrm{e}\rangle}\right) e^{-\kappa t / 2}$. Here, $Q_{|\mathrm{g}\rangle /|\mathrm{e}\rangle}$ are the measured readout resonator quadrature values of the steady states corresponding to $|\mathrm{g}\rangle$ and $|\mathrm{e}\rangle$. The resonator bandwidth $\kappa$ sets the response time $\tau_{B}=2 / \kappa$ of the measurement apparatus to a quantum jump - the decay time of the exponential evolution. 
The readout power, populating the readout resonator with $\bar{n}$ photons, and the integration time $\tau$ affect the SNR and hence determine whether measurement is weak or projective. A typical example of a sequence of weak measurements is shown in the middle panel of Fig. 4.2. There are two ways to convert this measurement to a strong one: by utilizing the "high- $\tau$ " regime obtained increasing $\tau$ (left panel in Fig. 4.2), or by utilizing the dynamical regime in which the required SNR is achieved by increasing $\bar{n}$ (right panel in Fig. 4.2).

Once the projective measurement regime is achieved, the next step is to minimize the state discrimination time. For the "high- $\tau$ " approach it can only be achieved by increasing the readout resonator coupling strength $\kappa$. Although being a viable solution, the $\kappa$ increase has a limit due to unwanted effects, such as cross-talk between different qubits, Purcell effect, and limitation of the frequency space required for multiplexing. Hence, in order to decrease the detection time further, one requires a complementary tuning knob.

A photon number increase is a viable solution, and as integration time decreases with increasing $\bar{n}$, the readout enters the dynamical regime in which the steady-state SNR doesn't depend on the integration time (Fig. 4.2(c)). However, until now, for the state-of-the-art readout in cQED this regime was not achieved: the typical readout power is $\bar{n}<10$, which is orders of magnitude below the expected critical photon number arising from the extra terms appearing in the James-Cummings Hamiltonian. When the drive power increases beyond the few photons limit, the readout becomes non-QND.

In this work I will present results of the high-power quantum jumps detection, made possible thanks to the grAl fluxonium readout staying QND up to $\bar{n}=150$ photons [GSG ${ }^{+} 21$ ]. First, the detection algorithm utilizing Bayesian filtering will be described in Sec. 4.3, which is essential for the dynamical regime since a standard latching filter cannot be used effectively for the quantum jumps detection. After that, the experimental results of the detection will be presented and discussed.

\subsection{Speed-up and detection}
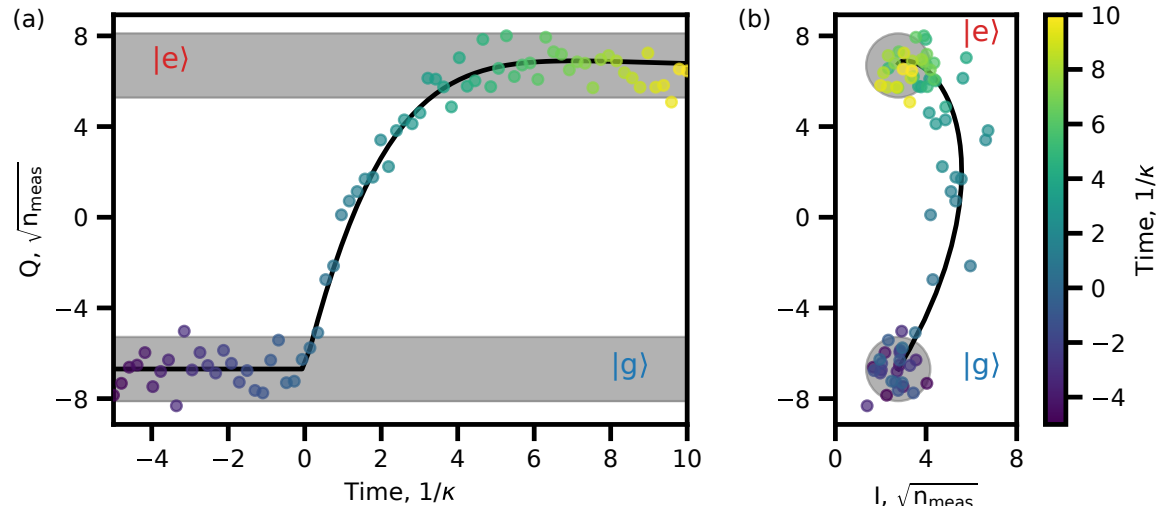

Figure 4.3: Example of a quantum jumps trace for the "high-power" readout regime. The simulation was made for $\bar{n}=100$, $\tau=0.2 / \kappa, \omega_{\text {drive }}-\omega_{|\mathrm{e}\rangle}=0.3 \kappa$. (a) Simulated Q quadrature in the units of measured photons as a function of time. Black line corresponds to the ideal (noiseless) classical trajectory. Grey areas indicate $\pm 2 \sigma$ intervals centered around the mean values of the $\mathrm{Q}$ quadrature corresponding to the ground and first excited state, respectively, denoted $Q_{|\mathrm{g}\rangle}$ and $Q_{|\mathrm{e}\rangle}$. The noise is corresponds to the noise of a quantum-limited readout chain. (b) Simulated IQ response. Grey circles indicate the $\pm 2 \sigma$ area. Color corresponds to time in (a).

For the $\tau$ increase approach illustrated in Fig. 4.2(a) the qubit state detection is straightforward. All measured data points are located around one of the resonator steady states, and one can use a simple latching filter declaring a jump when a pointer state enters the respective $2 \sigma$ area. 
In the dynamical readout regime, the physically instantaneous quantum jump is "delayed" due to the readout resonator response (see Fig. 4.3). As a result, the output signal is seemingly non-Markovian with a memory time $\tau_{B}=2 / \kappa$, which is a problem for the latching filter because it is resonator-response limited, and cannot infer the qubit state faster than $\tau_{B}$. However, at each point in time the state of the qubit is encoded in the instantaneous evolution of the pointer state, and is in fact Markovian. The full information about the quantum system can be extracted by asking a question "where does the response move?" instead of the latching-filter question "where is the response?".

In order to implement this kind of detection, a recursive Bayesian filter based on the hidden Markov model can be utilized. At each time point it updates the Bayesian probabilities $P\left(H \mid \alpha_{t+\tau}, \alpha_{t}\right)$, where $H$ is the qubit state hypothesis, and $\alpha_{t+\tau}, \alpha_{t}$ are the measured responses at the current $(t+\tau)$ and previous $(\mathrm{t})$ time points. The probabilities are getting updated according to the standard Bayesian formula

$$
P_{H}(t+\tau)=P(H \mid t+\tau)=\frac{P\left(\alpha_{t+\tau}, \alpha_{t} \mid H\right) \times P_{H}(t)}{\sum_{H^{\prime} \in\{\mathrm{g}, \mathrm{e}\}} P\left(\alpha_{t+\tau}, \alpha_{t} \mid H^{\prime}\right) \times P_{H^{\prime}}(t)}
$$

The unnormilized probability $P\left(\alpha_{t+\tau}, \alpha_{t} \mid H\right)$ to observe the measured evolution of the pointer state assuming the qubit is in state $H$ is calculated as

$$
P\left(\alpha_{t+\tau}, \alpha_{t} \mid H\right)=\exp \left[-\frac{\left|\alpha_{t+\tau}-\alpha_{t+\tau}^{\text {calc }}\left(\alpha_{t}, H\right)\right|^{2}}{2 \beta_{H}^{2} \sigma_{H}^{2}}\right] .
$$

This probability is a Gaussian with standard deviation $\beta_{H} \sigma_{H}$, where $\sigma_{H}$ is the variance of the pointer states, $\beta_{H} \in(0.8,1.2)$ is a coefficient tuning the filter responsivity, and

$$
\alpha_{t+\tau}^{\text {calc }}\left(\alpha_{t}, H\right)=\left(\alpha_{t}-A_{H}\right) e^{-\kappa \tau / 2+i\left(\omega_{\text {drive }}-\omega_{H}\right) \tau}+A_{H}
$$

is the pointer state corresponding to the classical trajectory starting at a pointer state with $\alpha_{t}$ and ending at the steady state $A_{H}$ corresponding to the qubit state hypothesis, similar to solid black lines in Fig. 4.3(b) corresponding to the classical trajectory between the steady states. The last expression also allows to estimate the actual signal-to-noise of the measurement: distance between the points of two different trajectories after time $\tau$ divided by noise. As a result, this can provide with a timescale for the time evolution of the discrimination between quantum states. However, one should keep in mind that each trajectory in the IQ space is different, depending on the readout frequency and power, and some of them might even intersect, especially when number of the quantum system states is higher than two.

If the angle between pointer states is big enough, it is also possible to make a more robust approximation and only track the phase (or a quadrature), assuming it evolves as

$$
\varphi_{t+\tau}^{\text {calc }}\left(\varphi_{t}, H\right) \approx\left(\varphi_{t}-\phi_{H}\right) e^{-C_{H} \kappa \tau / 2}+\phi_{H}
$$

Here $\varphi_{t}$ is the measured phase at time $t, \phi_{H}$ is the phase of the respective steady state, and $C_{H}=1 \pm 0.2$ an empirical coefficient accounting for the resonator nonlinearity and for the term $e^{i\left(\omega_{\text {drive }}-\omega_{H}\right) \tau}$ in Eq. (4.9). Both pointer state evolution (Eq. (4.9)) and exponential phase evolution (Eq. (4.10)) showed similar results when used for the Bayesian filtering in the experiment, and the simpler Eq. (4.10) was utilized to calculate the expected evolution for Eq. (4.8) in the experiment presented in the next two sections. 


\subsection{Setup and qubit characterization}

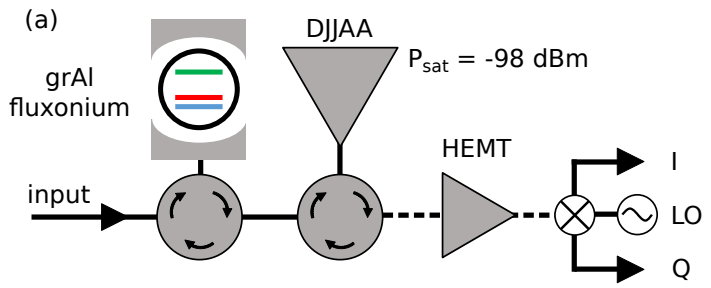

(c)

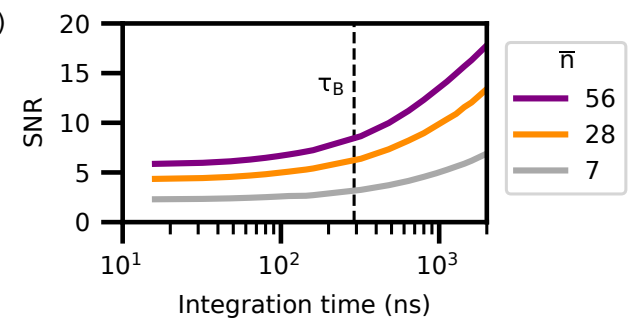

(b)

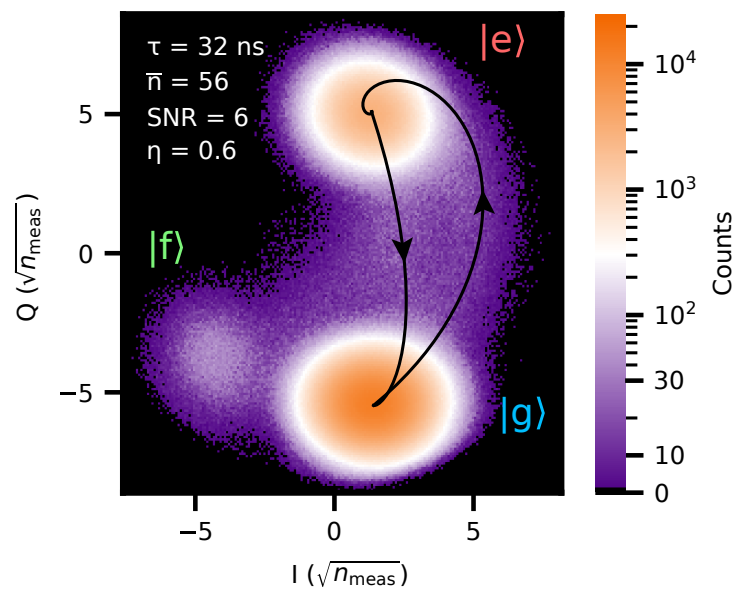

Figure 4.4: (a) Simplified schematic of the experimental setup. The incident readout signal reflects from a resonator coupled to a fluxonium artificial atom, both implemented using granular aluminum, which is the same device discussed in Ref.[GSG $\left.{ }^{+} 21\right]$. The reflected signal is amplified by a dimer Josephson junction array amplifier (DJJAA) [WTR ${ }^{+} 20$ ] operated in the non-degenerate phase-preserving regime. The measured $1 \mathrm{~dB}$ compression point of the DJJAA at $20 \mathrm{~dB}$ of power gain is $P_{\text {sat }}=-98 \mathrm{dBm}$ (see Sec. 3.3). The signal is further amplified by a commercial high electron mobility transistor amplifier (HEMT) thermalized at $4 \mathrm{~K}$, and is demodulated at room temperature into the I and Q quadratures. (b)2D histogram of continuously measured I and Q quadratures, presented in units of square root of measurement photons $\sqrt{\bar{n} B^{-1} \kappa / 4}$, where $\bar{n}=56$, and $B^{-1}=322 \mathrm{~ns}$. In order to highlight the resonator classical trajectories, the colorbar scale is linear below 30 counts and logarithmic above. The calculated trajectories $(|\mathrm{g}\rangle$ to $|\mathrm{e}\rangle$ and back) corresponding to the linear response of the resonator are indicated by the black traces. The signal-to-noise ratio, defined by Eq. (4.6), is SNR $=6$, and the obtained quantum efficiency for individual pointer state measurements is $\eta=0.6 \pm 0.1$. (c) Signal-to-noise ratio measured as a function of integration time $\tau$ for different $\bar{n}$ values. The black dotted line corresponds to $\tau_{\mathrm{B}}$.

In order to realize a dynamical continuous readout, one requires both a high dynamic range quantum-limited amplifier and an artificial atom resilient to high-power readout. A sketch of the experimental setup fulfilling these two conditions is shown in Fig. 4.4(a). The measured quantum system is a fluxonium artificial atom [MKGD09], dispersively coupled via a shared inductance to a readout resonator (see Table A.2 for the list of system parameters). The inductors required for both the fluxonium and readout antenna are implemented using grAl [GSG ${ }^{+}$19], and the fluxonium junction is implemented in the shape of a SQUID $\left[\mathrm{GSG}^{+} 21 ; \mathrm{LNG}^{+} 18 \mathrm{~b}\right]$. The sample chip was placed in a rectangular waveguide, following the concept in Ref.[KSV+18], and measured in reflection. The DJJAA described in section 3.3 was used to perform non-degenerate, phase-preserving amplification [RD16; $\mathrm{ESM}^{+} 14$ ] with $20 \mathrm{~dB}$ of power gain and $7 \mathrm{MHz}$ instantaneous bandwidth. The DJJAA saturation power was $-98 \mathrm{dBm}$, corresponding to a circulating photon number in the readout resonator $\bar{n} \approx 10^{4}$.

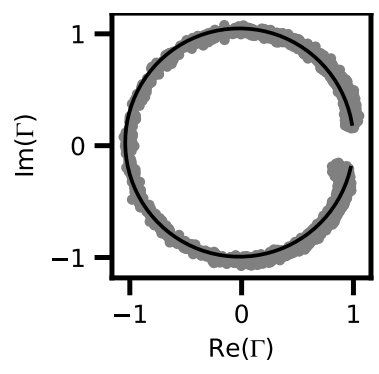

Figure 4.5: Normalized reflection (grey dots) and a circle fit result (black solid line). Probe frequency is the parameter of the plot. 
First, the reflection from the readout resonator is measured with VNA at low $(\bar{n} \ll 1)$ power (see Fig. 4.5 ). A circle fit $\left[\mathrm{PSB}^{+} 15\right]$ is applied to extract the resonator characteristics, namely resonance frequency $f_{\text {res }}=$ $7.247 \mathrm{GHz}$, coupling to the transmission line $\kappa=f_{\text {res }} / Q_{\mathrm{c}}=1.1 \mathrm{MHz}$, and the loss rate $\gamma=f_{\text {res }} / Q_{\mathrm{i}}=$ $20 \mathrm{kHz}$. Thanks to $\gamma \ll \kappa$, only a negligible part of the readout signal is lost due to dissipation.

After the readout resonator characterization, the flux bias for the fluxonium qubit was fine-tuned to $0.542 \Phi_{0}$ in the vicinity of the forth $E_{\mathrm{ge}}$ minimum $\left[\mathrm{GSG}^{+} 21\right]$ to maximize pointer states separation. For this minimum, the qubit parameters are: $E_{C} / h=2.8 \mathrm{GHz}, E_{L} / h=0.71 \mathrm{GHz}$. In Fig. 4.4(b) a typical histogram of the demodulated readout resonator response for $\bar{n}=56$ and an integration time $\tau=32 \mathrm{~ns}$ is plotted. The distribution shows three maxima, corresponding to the first three lowest energy levels of the fluxonium artificial atom, labeled $|\mathrm{g}\rangle,|\mathrm{e}\rangle,|\mathrm{f}\rangle$. Data initially measured in the units of volts on a digitizer is converted to the units of square root of measured photons $\sqrt{\bar{n} B^{-1} \kappa / 4}$, where $\bar{n}$ is the calibrated resonator population, and $B^{-1} \approx \tau+2 / \kappa$. Since the integration time $\tau=32 \mathrm{~ns}$ is much shorter than the characteristic response time of the measurement setup $\tau_{B}=290 \mathrm{~ns}$, trajectories corresponding to transitions between the three steady states are visible (see Fig. 4.6(a)), filling the space between the maxima in the IQ-plane. By measuring angles between steady states, qubit dispersive shifts $\chi_{\mathrm{ge}} / 2 \pi=-1.09 \mathrm{MHz}$ and $\chi_{\mathrm{ef}} / 2 \pi=1.46 \mathrm{MHz}$ were obtained following equation $\tan \left(\varphi_{i j} / 4\right)=\chi_{i j} / \kappa$. Temperature of the fluxonium, inferred from the population of ground and exited states assuming Boltzmann distribution, was $T_{\mathrm{q}}=\frac{\hbar \omega_{\mathrm{ge}}}{k \ln \left(N_{\mathrm{g}} / N_{\mathrm{e}}\right)}=31 \mathrm{mK}$. The measured rates of each transition at $\bar{n}=56: \Gamma_{\mathrm{eg}}=35 \mu \mathrm{s}, \Gamma_{\mathrm{ge}}=130 \mu \mathrm{s}, \Gamma_{\mathrm{ef}}=42 \mu \mathrm{s}, \Gamma_{\mathrm{fe}}=0.4 \mu \mathrm{s}$, were measured by fitting the histogrammed lengths of the corresponding quantum jumps with an exponential decay curve.

Quantum efficiency of the readout chain $\eta=0.6 \pm 0.1$ is the ratio of the noise photons of the standard quantum-limited chain ( with 1 total noise photon) and the measured one. It was extracted by fitting the steady-state SNR (Eq. (4.6)) as a function of integration time for different photon numbers (see Fig. 4.6(b)). The main source of uncertainty for the quantum efficiency is the photon number calibration.
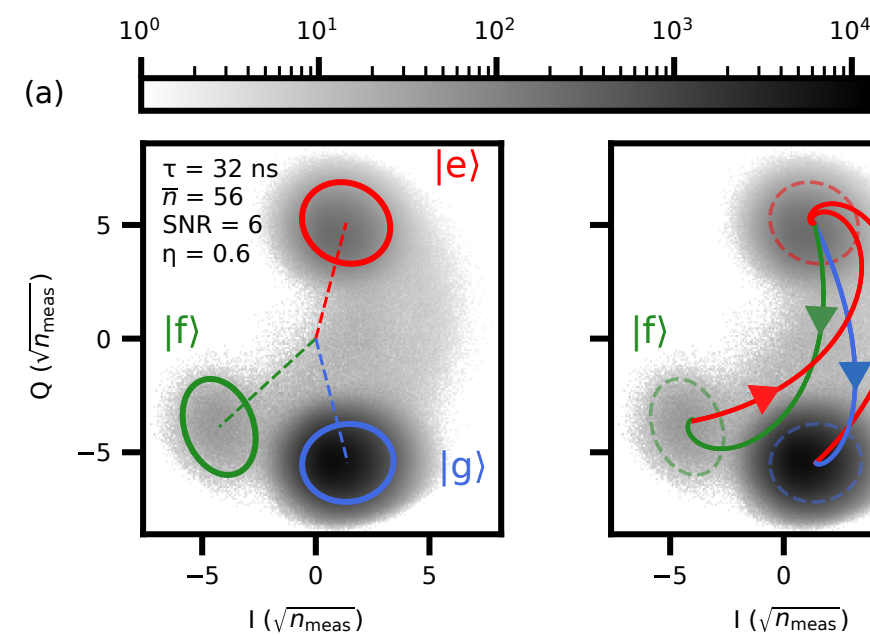

$10^{4}$

(a)

I $\left(\sqrt{n_{\text {meas }}}\right)$
Counts

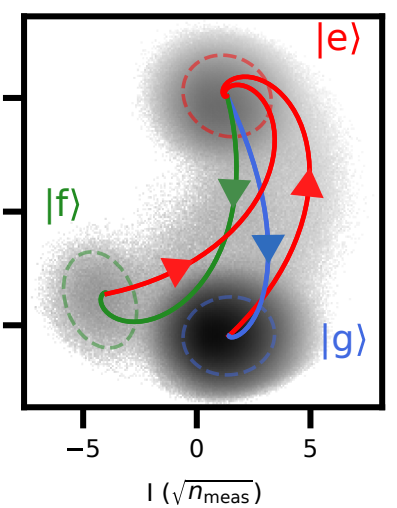

(b)
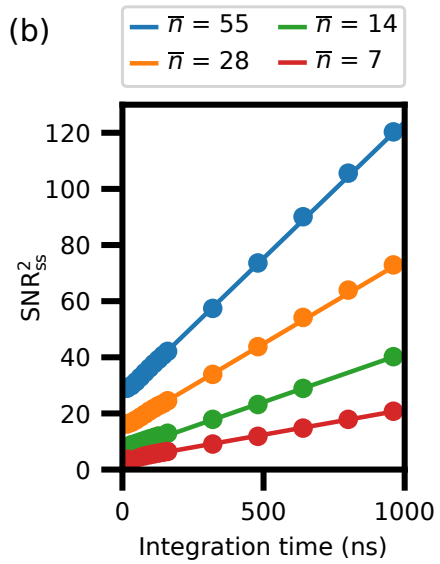

Figure 4.6: (a) 2D histograms of measured I and Q quadratures using the same data as in Fig. 4.4(c). Ellipses indicate the $2 \sigma$ areas for $|\mathrm{g}\rangle,|\mathrm{e}\rangle$, and $|\mathrm{f}\rangle$ steady states. Dotted lines on the left-hand panel show the location of steady state pointer states $\alpha_{i}$, with $i \in\{|\mathrm{g}\rangle,|\mathrm{e}\rangle,|\mathrm{f}\rangle\}$, and arrowed colored curves on the right figure indicate resonator classical trajectories to a steady state of corresponding color. (c) Squared steady-state signal-to-noise ratio (Eq. (4.6)) as a function of integration time for different $\bar{n}$. Straight lines are linear fits used to extract a measurement efficiency $\eta=0.6 \pm 0.1$, with the main source of uncertainty being the photon number calibration.

The fluxonium decay times $T_{1}=32.1 \pm 0.7 \mu \mathrm{s}$ and $T_{2}=0.73 \pm 0.06 \mu \mathrm{s}$ (see Fig. 4.7(c),(d)) were obtained following the standard routines illustrated in Figs. 4.7(a)(b). Before performing both experiments, the $\pi$ pulse (a pulse sent via the qubit drive line causing a $180^{\circ}$ rotation of the qubit state on the Bloch sphere) was calibrated by measuring the Rabi oscillations. For the $T_{1}$ measurement (see Fig. 4.7(c)), after sending 
a $\pi$ pulse via the qubit control line and waiting for a delay, a qubit state readout is performed. By obtaining averaged results of multiple measurements for a number of delay times, one gets a decaying exponential corresponding to a free decay of a qubit form exited to ground state. Procedure for the Ramsey $T_{2}$ experiment is similar: first, a $\pi / 2$ pulse is sent, followed by a delay, a second $\pi / 2$ pulse, and a qubit state readout.



(b)

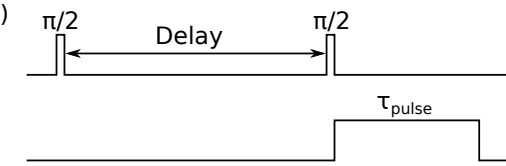

(c)

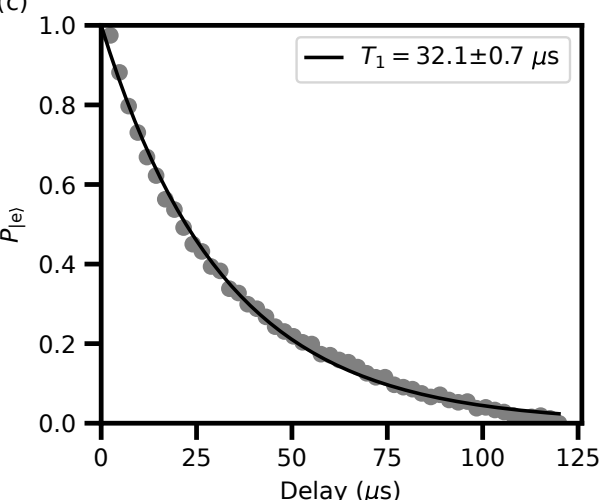

(d)

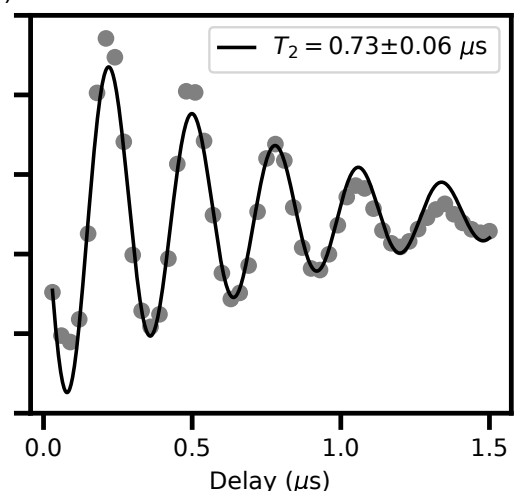

Figure 4.7: Qubit coherence measurements: $T_{1}(\mathrm{a}, \mathrm{c})$ and $T_{2}$ (Ramsey coherence time). (a),(b) Diagrams of the pulse sequences. Upper traces indicate pulses at a qubit frequency, lower — at a readout frequency. (c) Measured $T_{1}$ trace (grey dots). Black line is a numerical fit of an exponential decay. (c) Measured $T_{2}$ trace (grey dots). Black line is a numerical fit of a damped sine.

(a)

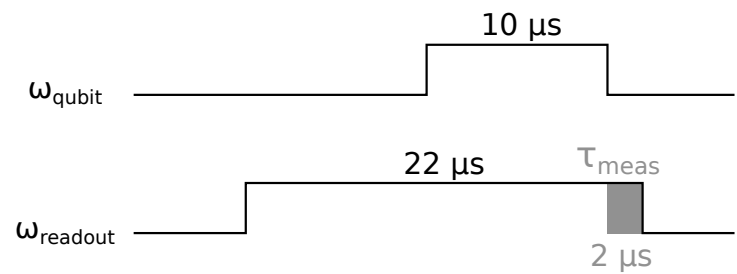

(b)

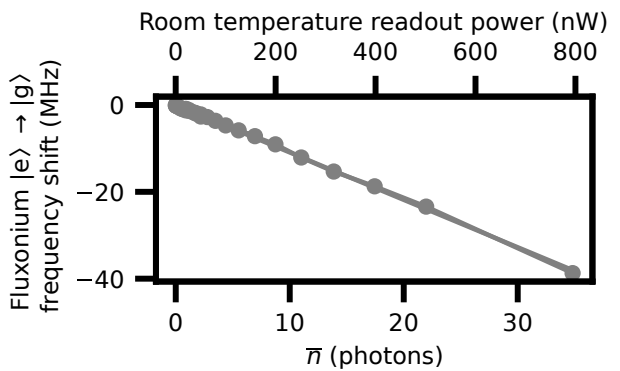

Figure 4.8: (a) Pulse sequence used for the ac Stark measurement. Upper trace indicates pulse at a qubit frequency , lower — at a readout frequency. (b) Measured ac-Stark shift plotted versus room temperature readout power (top axis) and the corresponding calibrated photon number (bottom axis).

To calibrate the readout power, ac Stark effect was used. Since fluxonium and resonator states are hybridized, not only resonator frequency is qubit state dependent, but also the qubit frequency depends on the resonator population, following

$$
f_{\mathrm{ge}}(\bar{n})=f_{\mathrm{q}}(0)+\chi_{\mathrm{ge}} \bar{n}
$$

Hence, by measuring the power dependence of the qubit frequency shift one can extract attenuation between power the room-temperature microwave generator and input of the fluxonium sample. To measure this, the pulse sequence shown in Fig. 4.8(a) was used. First, a $20 \mu$ s long $\left(\tau_{\text {pulse }} \gg 2 / \kappa\right)$ readout tone is applied. In the middle of the readout pulse, a $10 \mu$ s long qubit drive is sent. Since the duration of the drive is much longer than the Rabi decay time, in the end of the pulse the qubit is in the mixed state with $P_{\mathrm{e}}=0.5$. In the 
end, right after the end of the manipulation pulse, the state of the qubit is recorded, indicated by the grey area on the Fig. 4.8(a).

A more precise method of photon number calibration, the measurement-induced dephasing [BTH $\left.{ }^{+} 18\right]$, was not possible for our setup because $T_{2}$ was limited to sub-us range due to being susceptible to local noise even when operated at a sweet spot for global flux noise.

\subsection{Bayesian detection applied}

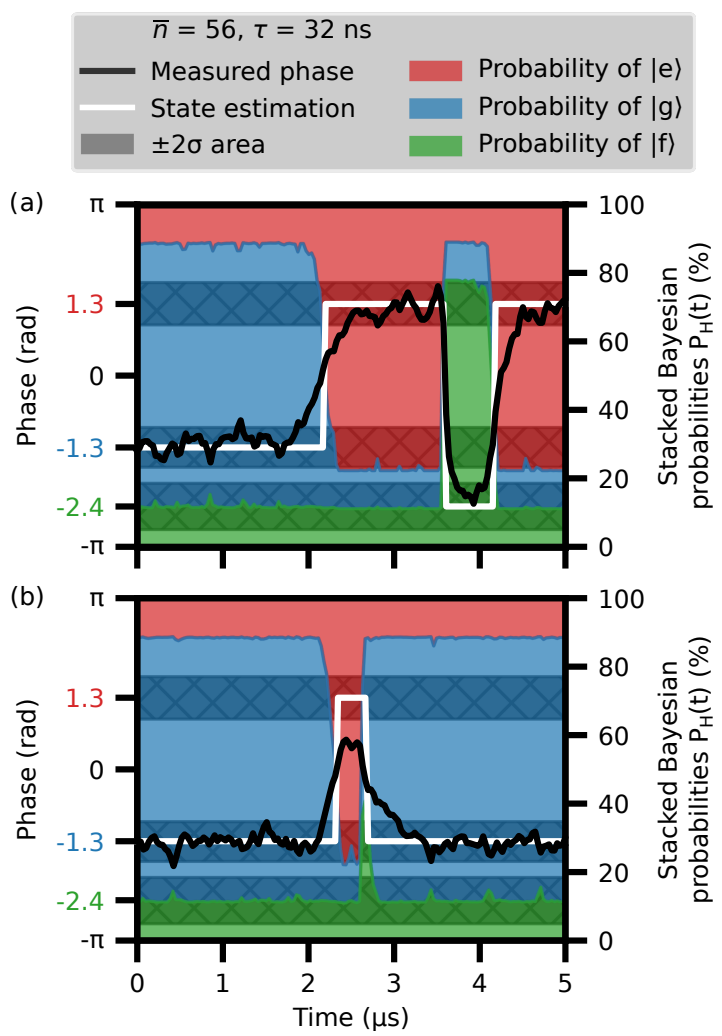

Figure 4.9: Examples of Bayesian inference of the artificial atom's state $(|g\rangle,|e\rangle$, or $|\mathrm{f}\rangle)$ based on the continuous wave measurement of the readout resonator. Black lines represent the phase vs. time extracted from the measured IQ response (see Fig. 4.4(b)) for $\bar{n}=56$ and $\tau=32 \mathrm{~ns}$. Hatched areas indicate the $\pm 2 \sigma$ intervals for each state, centered on the corresponding average phase response: -1.3 for $|\mathrm{g}\rangle$ (blue label), 1.3 for $|\mathrm{e}\rangle$ (red label), and -2.4 for $|\mathrm{f}\rangle$ (green label). Colored areas show the stacked barplot vs. time for the calculated Bayesian probabilities (see Eq. (4.7)) of the first three states of the artificial atom: $|\mathrm{g}\rangle$ in blue, $|\mathrm{e}\rangle$ in red, and $|\mathrm{f}\rangle$ in green. The inferred quantum state is indicated by the white line; when one of the Bayesian probabilities reaches $50 \%$, a jump to the respective state is declared.

After the system calibration and characterization, measurements of quntum jumps with drive power up to $\bar{n}=110$ were performed. Thanks to the increased SNR, a Bayesian inference can be implemented for quantum jumps detection, allowing to extract the state of the fluxonium. Typical detection sequences for quantum jumps between the $|\mathrm{e}\rangle,|\mathrm{g}\rangle$, and $|\mathrm{f}\rangle$ fluxonium states using the recursive Bayesian filter are shown in Fig. 4.9. Based on the measured black trace, the filter estimate for the fluxonium state is indicated by the white trace. Notice that the $|\mathrm{e}\rangle \rightarrow|\mathrm{f}\rangle$ and $|\mathrm{f}\rangle \rightarrow|\mathrm{e}\rangle$ quantum jumps are correctly identified, even though the pointer state intersects the phase value corresponding to the $|\mathrm{g}\rangle$ steady state. A quantum jump is declared once one of the probabilities surpasses $50 \%$. The minimum probability for each state is typically capped at $10 \%$ to prevent the filter saturation at $P_{H}(t)=0$, in which case Eq. (4.7) is no longer responsive. In Fig. 4.9(b) I 

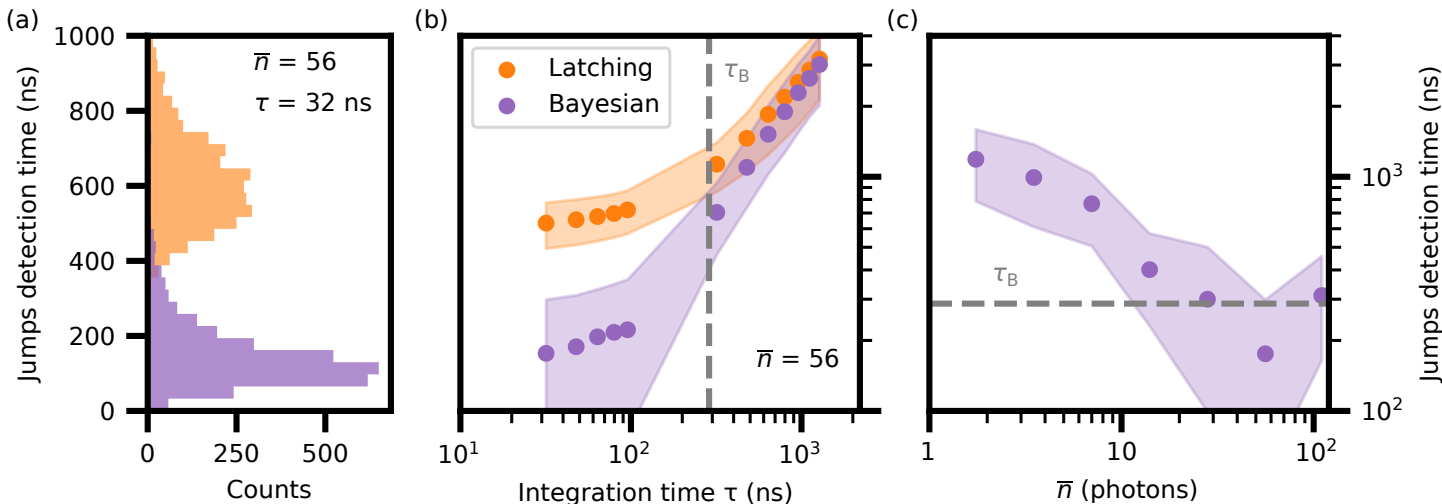

Figure 4.10: (a) Histograms of the quantum jump detection times obtained with a three-point latching filter (orange), and a recursive Bayesian filter (purple, see Eq. 4.7), for $|\mathrm{g}\rangle \rightarrow|\mathrm{e}\rangle$. For this histogram $\bar{n}=56$, and the integration time is $\tau=32 \mathrm{~ns}$. (b) Average detection time for $|\mathrm{g}\rangle \rightarrow|\mathrm{e}\rangle$ jumps as a function of integration time, obtained using the three-point latching filter (in orange) or the recursive Bayesian filter (in purple). Colored areas represent the standard deviation of the jump detection time distribution (see panel a). (c) Quantum jumps detection time as a function of photon number in the readout resonator $\bar{n}$, for $|\mathrm{g}\rangle \rightarrow|\mathrm{e}\rangle$ quantum jumps. The colored area represents the standard deviation of the jump detection time distribution (see panel a).

selected an example which illustrates the ability of the Bayesian filter to declare a $|\mathrm{g}\rangle \rightarrow|\mathrm{e}\rangle \rightarrow|\mathrm{g}\rangle$ quantum jumps sequence for which the readout resonator never reached the steady state associated with $|\mathrm{e}\rangle$.

In Fig. 4.10 the Bayesian state estimate is compared to a latching filter for $|\mathrm{g}\rangle \rightarrow|\mathrm{e}\rangle$ transition. The latching filter was designed to declare a jump when a pointer state enters the $2 \sigma$ area of a respective steady state. The detection time (for both latching and Bayesian filtering) is defined as time between leaving the $2 \sigma$ area of the previous coherent state and jump detection. In Fig. 4.10(a), histograms of the jump detection times are shown for $\tau=32 \mathrm{~ns}$. The Bayesian inference provides a 3.5 times faster state discrimination on average. In Fig. 4.10(b), the mean jump detection time is shown versus $\tau$ for both filters. As expected, the Bayesian detection starts to outperform the latching filter for $\tau<\tau_{\mathrm{B}}$ (also see Fig. 4.11). The obtained measurement QND fidelity [TKF $\left.{ }^{+} 19\right]\left(P_{\mathrm{e} \mid \mathrm{e}}+P_{\mathrm{g} \mid \mathrm{g}}\right) / 2=98 \%$, where $P_{\mathrm{e} \mid \mathrm{e}}$ and $P_{\mathrm{g} \mid \mathrm{g}}$ are the probabilities to obtain the same result in consecutive measurements separated by $\Delta t$, is comparable to state-of-the-art $\left[\mathrm{JSM}^{+} 14 ; \mathrm{KBS}^{+} 16 ; \mathrm{WKG}^{+} 17 ; \mathrm{TKF}^{+}\right.$19; $\mathrm{DRM}^{+}$20]. The time interval $\Delta t=432 \mathrm{~ns}$ is chosen such that the detection time of $98 \%$ of all quantum jumps is less than $\Delta t$ for the Bayesian filter. Transitions from $|\mathrm{e}\rangle$ to $|\mathrm{g}\rangle$ and $|\mathrm{f}\rangle$ during $\Delta t$ contribute $1 \%$ and $1.2 \%$, respectively, to the value of $P_{\mathrm{e} \mid \mathrm{e}}=96.7 \%$, and transitions from $|\mathrm{g}\rangle$ to $|\mathrm{e}\rangle$ contribute $0.3 \%$ to the measured $P_{\mathrm{g} \mid \mathrm{g}}=99.6 \%$.

In Fig. 4.10(c), the average jump detection time for the $|\mathrm{e}\rangle \rightarrow|\mathrm{g}\rangle$ transition is shown as a function of $\bar{n}$. The results are obtained using the recursive Bayesian filter since the latching filter detecton time saturates at $620 \mathrm{~ns}$ (see Fig. 4.10(b)). The detection time decreases with $\bar{n}$, as expected from Eq. (4.5), and it has a minimum of $175 \mathrm{~ns}$ for $\bar{n}=56$. The increase at $\bar{n}=110$ is caused mainly by the nonlinearity of the grAl readout resonator and the associated squeezing of the pointer state distributions discussed in the next section. 


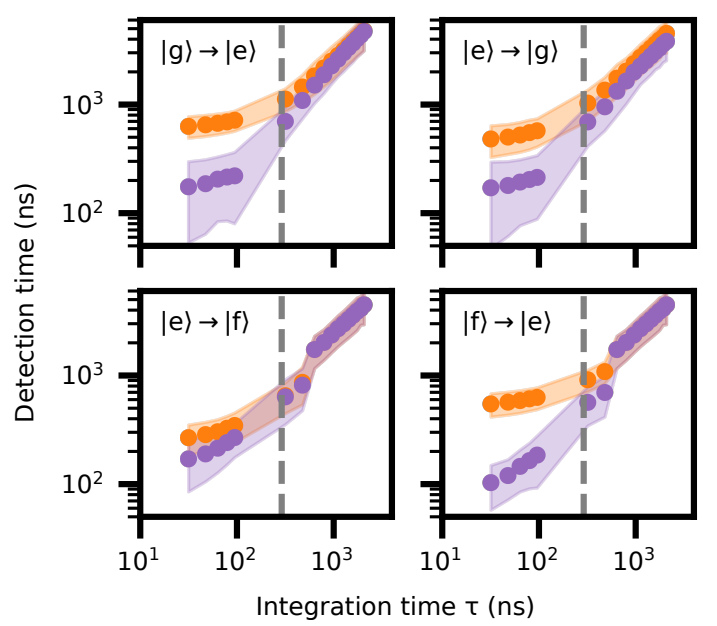

Figure 4.11: Average detection time for $|\mathrm{g}\rangle \rightarrow|\mathrm{e}\rangle,|\mathrm{e}\rangle \rightarrow|\mathrm{g}\rangle,|\mathrm{e}\rangle \rightarrow|\mathrm{f}\rangle,|\mathrm{f}\rangle \rightarrow|\mathrm{e}\rangle$ jumps as a function of integration time, obtained using the three-point latching (in orange) or the recursive Bayesian filter (in purple). Transparent areas represent the standard deviation of the jump detection time distribution. The grey dashed line corresponds to $\tau_{\mathrm{B}}$.

\subsection{Steady-state squeezing}

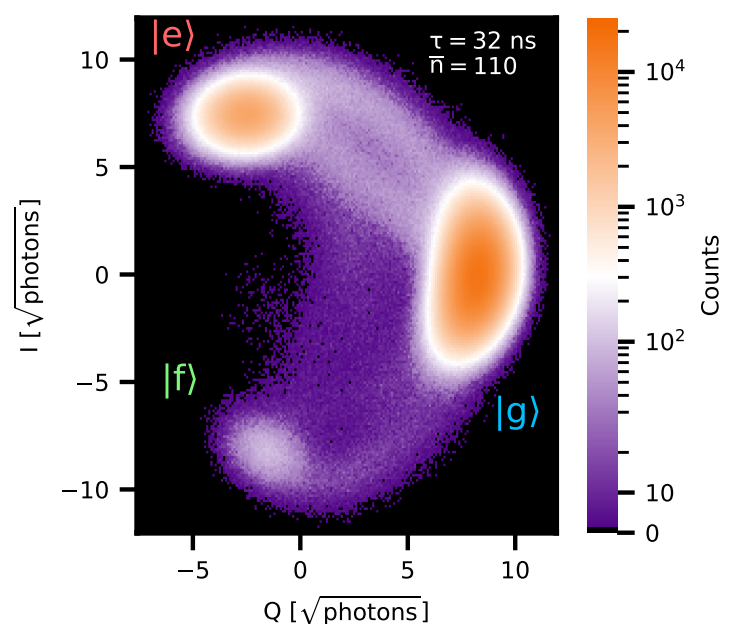

Figure 4.12: 2D histogram of measured I and Q quadratures for $\bar{n}=110$.

The steady-states in Fig. 4.4(b) are visibly squeezed, which becomes even more visible when the readout strength increases (see Fig. 4.12). This happens due to both the grAl readout resonator's intrinsic nonlinearity and the inherited state-dependent nonlinearities (see section 5.2). The intrinsic nonlinearity $K_{\text {grAl }} / 2 \pi=$ $-2.4 \mathrm{kHz}$ was measured by detuning the fluxonium from the hybridization flux interval and measuring the resonance frequency power dependence (see Fig. 4.13). The inherited state-dependent nonlinearities $K_{|\mathrm{g}\rangle} / 2 \pi=-2.6 \mathrm{~Hz}, K_{|\mathrm{e}\rangle} / 2 \pi=2 \mathrm{kHz}$, and $K_{|\mathrm{f}\rangle} / 2 \pi=-2 \mathrm{kHz}$ at $\bar{n}=50$ were calculated using [SKV $\left.{ }^{+} 16\right]$ thanks to the known fluxonium parameters (see Table A.2). Notice that for the $|e\rangle$ state the Kerr coefficients almost cancel out, as confirmed by the reduced squeezing of the $\mid$ e $\rangle$ pointer state in Fig. 4.4(c).

The bifurcation photon number corresponding to the intrinsic nonlinearity is $\bar{n} \approx 160$, resulting in a $K \bar{n} \sim \kappa$ regime for a readout tone creating resonator population of $\bar{n}=20-100$ photons. Hence, the readout tone 

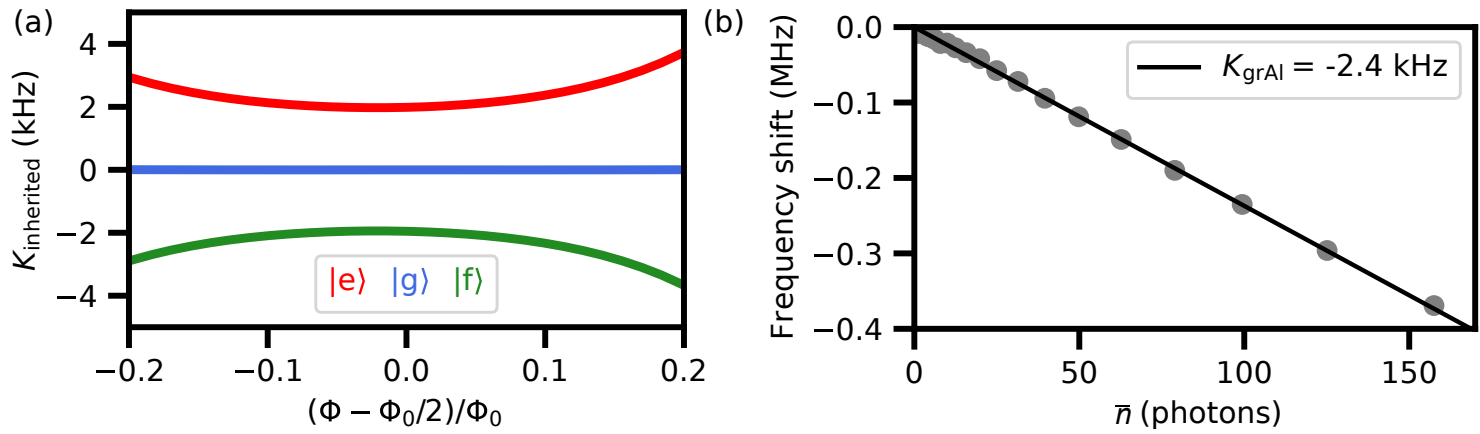

Figure 4.13: (a) Fluxonium-state-dependent inherited nonlinearities of the readout resonator as a function of external magnetic flux (b) Readout resonator frequency shift versus calibrated photon number.

(a)

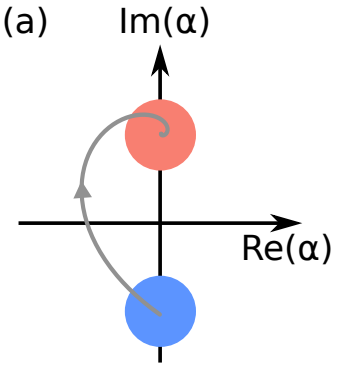

(b)

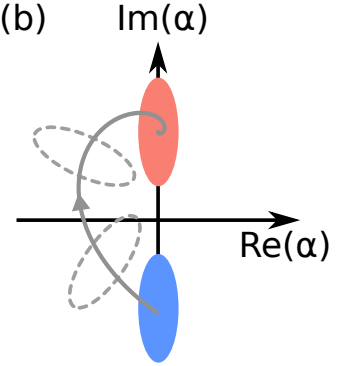

(c) $\operatorname{Im}(\alpha)$

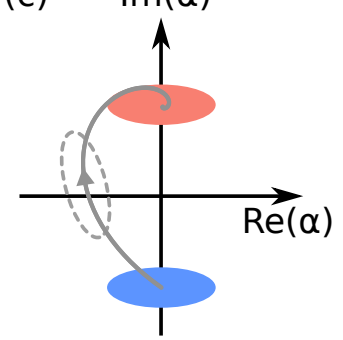

Figure 4.14: Sketches of the pointer states of a readout resonator coupled to a two-level system. Red color pointer states correspond to the excited state, and blue to the ground. The grey arrowed line depicts a classical trajectory from ground to excited state. The pointer state is either coherent (a), or squeezed (b) and (c), where the squeezing axis is aligned with the imaginary and the real axis, respectively, depending on the readout frequency and readout power. The grey dashed ellipses on (b) and (c) illustrate rotation of the squeezing axis of the pointer state during its evolution along the readout resonator classical trajectory.

effectively acts as a pump and creates parametric amplification with non-negligible gain (see Fig. 4.15(b)), which, depending on pump parameters, can reach up to $20 \mathrm{~dB}$ (see Appendix A.2.2). However, since the readout drive is the only applied tone, the only visible effect of the associated amplification is squeezing of the noise at the output of the qubit sample. Due to squeezing the effective readout SNR can, generally speaking, either increase or decrease. For the situation shown in Fig. 4.14(a), when the $|\mathrm{g}\rangle \rightarrow|\mathrm{e}\rangle$ trajectory is mostly visible in the phase response, states separation can be enhanced if the steady states are squeezed along the imaginary axis (Fig. 4.14(b)). On the other hand, squeezing along the real axis decreases the effective SNR (Fig. 4.14(c)).

The model system which can be used to calculate orientation of the squeezing axis and the quadraturedependent gain is a 4-wave-mixing JPA with resonance frequency and nonlinearity both being fluxoniumstate-dependent. A constant pump tone (our readout drive) is applied with fixed power and frequency. The only signal applied to the system is the vacuum noise, and the only noise measured in the experiment is the noise at the drive frequency. Thus we only need to consider the phase-sensitive regime with $\omega_{s}=\omega_{i}=\omega_{p}$. For phase-sensitive amplification, input and output fields are linked via

$$
b_{\text {out }}=g b_{\text {in }}+m b_{\text {in }}^{+} \text {, }
$$

where $g$ and $m$ are defined in Eq. (2.61). Both of them are complex functions, and depend on the detuning of the pump frequency from the bere resonance frequency $\omega_{p}-\omega_{0}$, coupling strength $\kappa$, loss rate $\gamma$, Kerr nonlinearity $K$, and pump frequency and power. On top of that, as can be seen from Eq. (2.61), 
(a)

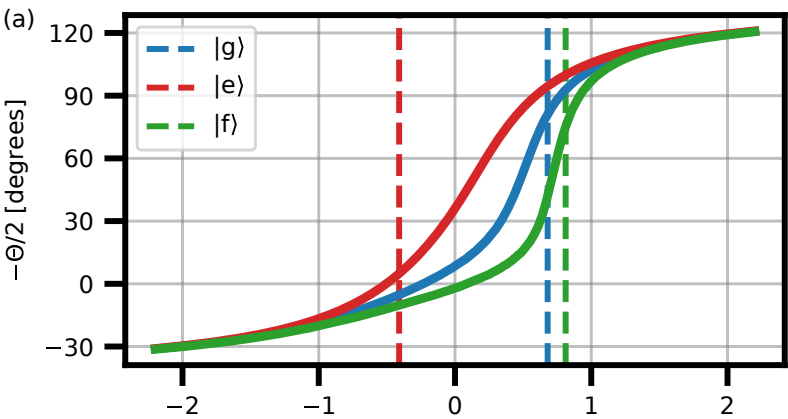

(b)

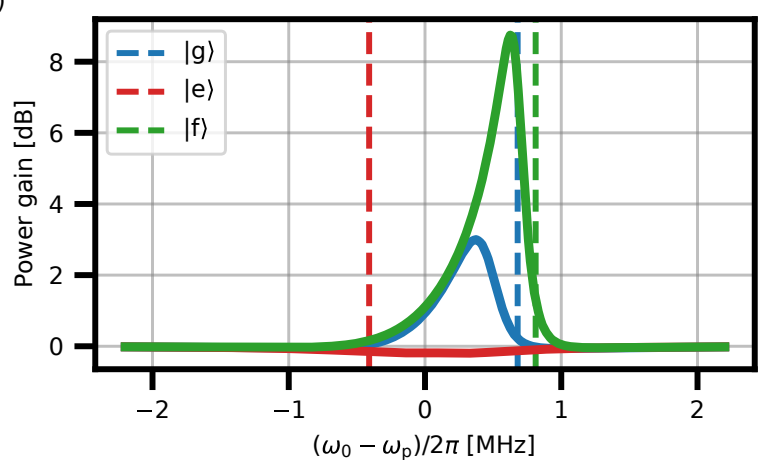

(c)

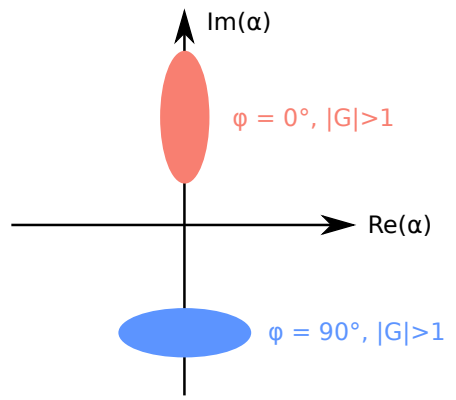

(d)

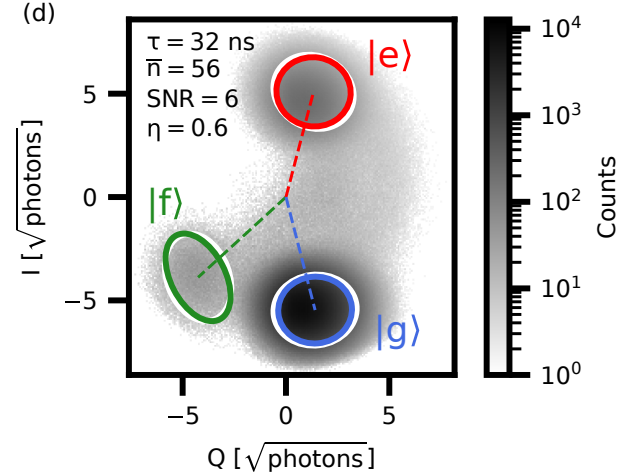

Figure 4.15: (a),(b) Theoretical prediction of dependence of squeezing axis angle and phase-sensitive gain versus detuning of the steady-state resonance frequency from the pump frequency for $\bar{n}=56$. Red, blue, and green curves correspond to $|\mathrm{g}\rangle$, $|e\rangle$, and $|f\rangle$ steady states. Respective vertical dotted lines indicate corresponding resonance steady states frequencies. (c) Schematic illustrating how squeezed states appear on the IQ plane for $\varphi=0$ deg (red ellipse) and $\varphi=90$ deg (blue ellipse) provided $G>1$. (d) 2D histogram of measured I and Q quadratures using the same data as in Fig. 4.3. White ellipses indicate the measured $2 \sigma$ areas for $|\mathrm{g}\rangle,|\mathrm{e}\rangle$, and $|\mathrm{f}\rangle$ steady states. Colored ellipses show the theoretical prediction of the squeezed noise.

$m$ also depends on the pump phase $\varphi_{p}$ while $g$ is not affected by it. By defining quadrature as $X_{\varphi}=$ $\frac{1}{2}\left(e^{i \varphi} b+e^{-i \varphi} b^{+}\right)$, one can find expression for $X_{\varphi}^{\text {out }}$ :

$$
X_{\varphi}^{\text {out }}=\frac{1}{2}\left(g e^{i \varphi}+m e^{-i \varphi}\right) b_{\text {in }}+\text { h.c. }
$$

By introducing $A=g\left(\omega_{p}\right) e^{i \varphi}+m^{*}\left(\omega_{p}\right) e^{-i \varphi}$, the last equation can be rewritten as

$$
X_{\varphi}^{\text {out }}=\frac{|A|}{2}\left(e^{i \varphi_{A}} b_{\text {in }}+e^{-i \varphi_{A}} b_{\text {in }}^{+}\right)=|A| X_{\varphi_{A}}^{\text {in }}
$$

where the quadrature gain is

$$
G_{\varphi}=|A|=\left|g e^{i \varphi}+m e^{-i \varphi}\right|=\sqrt{|g|^{2}+|m|^{2}+2|g m| \cos (2 \varphi+\Theta)} .
$$

Here $\Theta=\arg (g)-\arg \left(m^{*}\right)$ is the angle between $g$ and $m$. This angle, defining the direction of squeezing axis, depends on pump frequency, power, and phase. The quadrature corresponding to $\varphi=-\Theta / 2$ is amplified by $2|g|$ (provided $|g| \gg 1$ ), and the orthogonal quadrature corresponding to $\varphi=\pi / 2-\Theta / 2$ is amplified by $1 / 2|g|$ (squeezed). These quadratures are the eigenvectors of linear transformation in Eq. (4.14). Any other quadrature (linear combination of the squeezed and anti-squeezed ones) is not only amplified, but also gets rotated in the phase space $\left(X_{\varphi_{A}}^{\text {in }} \rightarrow X_{\varphi}^{\text {out }}\right)$.

In our experiment the pump frequency and power are fixed. Thus orientation of squeezing axis and gain depend on two fluxonium-state-dependent parameters: bare(non-Kerr-shifted) resonance frequency $\omega_{0} / 2 \pi$ 
and the Kerr coefficient of the JPA, as shown in Fig. 4.15(a,b). Three lines in Fig. 4.15(a,b) correspond to different fluxonium states $(|\mathrm{g}\rangle,|\mathrm{e}\rangle,|\mathrm{f}\rangle)$ and show how the anti-squeezing quadrature gain depend on the JPA bare frequency.

Table 4.1: Calculated gain and squeezing axis rotation angle for first three fluxonium states.

\begin{tabular}{|c|c|c|}
\hline State & $\varphi=-\Theta / 2$, degrees & $|G|^{2}, \mathrm{~dB}$ \\
\hline$|\mathrm{g}\rangle$ & 81 & 0.22 \\
$|\mathrm{e}\rangle$ & 5 & -0.16 \\
$|\mathrm{f}\rangle$ & 75 & 1.28 \\
\hline
\end{tabular}

Theoretical prediction of gain and squeezing axis angle for each state is listed in Table 4.1. For all three states, squeezing is close to tangential (note that $\left|G_{\mathrm{e}}\right|<1$ due to non-zero intrinsic losses of the readout resonator). Comparison between measured and calculated squeezed noise for $\bar{n}=56$ is shown in Fig. 4.15(d). As is evident, measured orientation of squeezing axis and quadrature gain are in qualitative agreement with the theoretical prediction. 


\section{Towards parametric amplifier resilient to magnetic fields up to few Tesla}

Josephson parametric amplifiers are nowadays an essential element in the readout chain for superconducting qubits. However, the Josephson junctions used to make these amplifiers suffer when exposed to high magnetic fields, which makes it very hard (if not impossible) to fully profit from them for a number of applications, such as kinetic inductance detectors, axion search, and readout of the state for hybrid structures. In this section I present a granular aluminum parametric amplifier (GrAPA) operating in degenerate four-wave-mixing regime, which was resilient to in-plane fields up to 1 Tesla.

\subsection{Granular Aluminum (grAl)}

Evaporation of aluminum in oxygen atmosphere results in the formation of a material consisting of crystalline grains with diameter of 2-4 nm embedded in an amorphous and non-stoichiometric aluminum oxide matrix. This material, refereed to as granular aluminum (grAl), is superconducting [ACC66; DGGI73]. On top of that, the grAl critical temperature is higher than the one of pure aluminum. Although this material was discovered more than 50 years ago, the exact mechanism of superconductivity and the increased critical temperature is still an object of theoretical research. Despite the lack of full understanding of the physics behind it, grAl possess a number of useful properties which make it attractive for numerous applications in cQED.

Thanks to its low losses, it can be used for quantum information processing. It was demonstrated that resonators made of grAl have $Q_{i} \sim 10^{5}$ at low photon numbers [RSP${ }^{+} 17$; $\left.\mathrm{GMS}^{+} 18\right]$ with losses dominated by quasiparticles $\left[\mathrm{SDR}^{+} 12\right]$ which can be minimized by e.g. utilizing phonon $\left[\mathrm{HVC}^{+} 19 ; \mathrm{VHC}^{+} 19\right]$ or quasiparticle $\left[\mathrm{HRS}^{+}{ }^{17}\right]$ traps. GrAl is a kinetic inductance material, with inductance as high as few $\mathrm{nH} / \square$, allowing to use granular aluminum as a source of compact inductance. Its kinetic inductance follows[Tin04; $\operatorname{RSP}^{+}$17]:

$$
L_{\square}=\frac{R_{\square} \hbar}{\pi \Delta},
$$

where $L_{\square}$ is the kinetic inductance per square, $R_{\square}=\rho_{\mathrm{n}} / d$ is the normal-state sheet resistance of the film, and $\Delta$ is the grAl superconducting gap. The resistivity $\rho_{\mathrm{n}}$ is controlled by the oxygen pressure during the $\mathrm{Al}$ evaporation, and is hence a convenient tuning knob allowing to create grAl structures with the desired kinetic inductance.

Kinetic inductance comes with Kerr nonlinearity, and grAl can be utilized as a nonlinear media with Kerr coefficient ranging from few $\mathrm{Hz}$ ( a 'linear inductor' regime ) to few $\mathrm{MHz}\left[\mathrm{MGK}^{+} 18\right]$. Owing to its kinetic inductance and nonlinearity, grAl was used for building linear inductors of superconducting qubits $\left[\mathrm{GSG}^{+} 19\right]$, for kinetic inductance detectors $\left[\mathrm{VHC}^{+} 19\right]$, and even as a nonlinearity source for a transmon qubit $\left[\mathrm{WBG}^{+} 20 ; \mathrm{SVW}^{+}\right.$20]. However, so far granular aluminum has not been utilized as a nonlinear media for parametric amplifier, for which one typically needs low losses and nonlinearity of $K \sim 1-100 \mathrm{kHz}$, both achievable for grAl structures. On top of the mentioned characteristics, grAl is resilient to in-plane 
magnetic fields up to few Tesla [BRW ${ }^{+}$20]. Thanks to this feature, a grAl-based parametric amplifier, which I refer to as GrAPA, can be used for high-magnetic-field applications, such as axion search [IR18] or readout of state for hybrid architectures $\left[\mathrm{BPK}^{+} 16\right]$.

\subsection{Nonlinearity of a grAl strip}
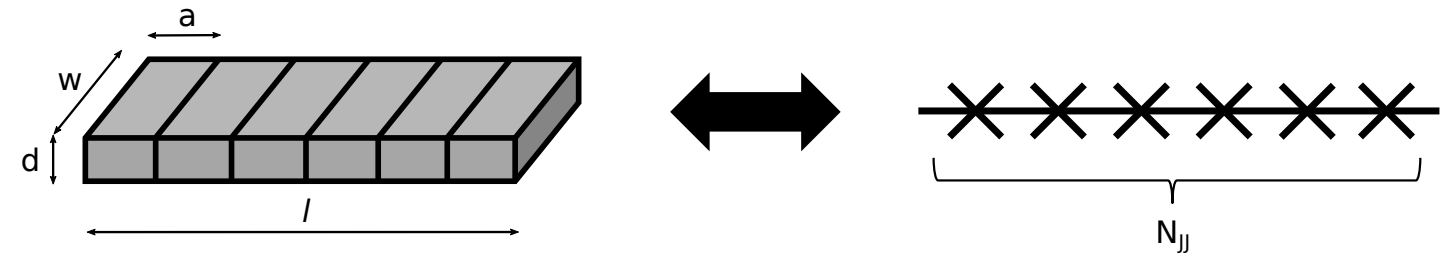

Figure 5.1: Sketch of a grAl strip (left panel) modelled as an array of Josephson junction array (right panel). 1,w,d are the dimensions of the strip, and a is the length corresponding to one effective Josephson junction.

For GrAPA design, it is important to be able to predict both kinetic inductance and nonlinearity of a grAl strip (the influence of the strip self-capacitances is negligible for the design used in this thesis). While grAl kinetic inductance is given by Eq. (5.1) and is confirmed by numerous experiments, calculating nonlinearity is a more complicated matter due to the non-trivial nature of granular aluminum. It was suggested in Ref.[MGK ${ }^{+}$18] to model a strip of grAl as a Josephson junction array (JJA). A strip (see Fig. 5.1) is effectively divided in "blocks" with length $a$, which in Ref. $\left[\mathrm{MGK}^{+} 18\right]$ was chosen to be equal to the Al grain diameter. While results of this work are in a great qualitative agreement with the measured data (see Fig. 5 in the $\left.\left[\mathrm{MGK}^{+} 18\right]\right)$, there is a quantitative mismatch of about an order of magnitude. This mismatch, as will be further showed in this section, is most likely present due to the choice of a.

Assuming modelling the strip with an effective JJA is correct, the model has to fulfill two conditions:

- The critical current of the Josephson junctions is equal to the critical current of the grAl strip $I_{c}^{\mathrm{grAl}}=I_{c}^{\mathrm{JJ}}$.

- The number of Josephson junctions is such that the total inductance of the grAl strip and the equivalent array are equal, $L_{\text {total }}^{\mathrm{grAl}}=N_{\mathrm{JJ}} L_{\mathrm{JJ}}$.

Thus the effective $\mathrm{JJ}$ size is

$$
a=l / N_{\mathrm{JJ}}=\frac{\pi \Delta}{2 e} \frac{1}{\rho_{\mathrm{n}} j_{\mathrm{c}}}
$$

The normal-state resistivity can be inferred from the room-temperature resistance measurement, and is hence an easy-to-get parameter. The critical current density, however, has to be extracted from experimental data since it does not follow the Mattis-Bardeen formula. It depends only on $\rho_{\mathrm{n}}$, and this dependence, shown on Fig. 5.2, was reported in $\left[\mathrm{FWB}^{+} 19\right]$. The dependence is not linear $\left(j_{\mathrm{c}} \propto \rho_{\mathrm{n}}^{-1.3}\right)$, and the effective JJ size is thus resistivity-dependent (see Fig. 5.3(a)), increasing from 10 to $70 \mathrm{~nm}$ as film resistivity is varied between oxygen-poor and oxygen-rich films. This prediction is in agreement with Ref. [YGS ${ }^{+} 20$ ], where authors show that for $\mathrm{k} \Omega$ grAl films, $\mathrm{Al}$ grains tend to form clusters.

For the Kerr coefficient calculation, lets consider a simple system consisting of a grAl strip shunted with a capacitor, similar to the grAl transmon qubit discussed in $\left[\mathrm{WBG}^{+} 20\right]$ (calculations for other resonator 


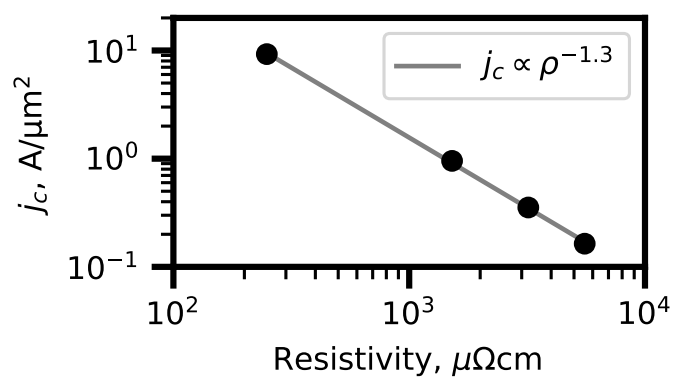

Figure 5.2: Critical curret density of grAl thin films versus the film normal-state resistivity. Adapted from $[\mathrm{FWB}+19]$.

(a)

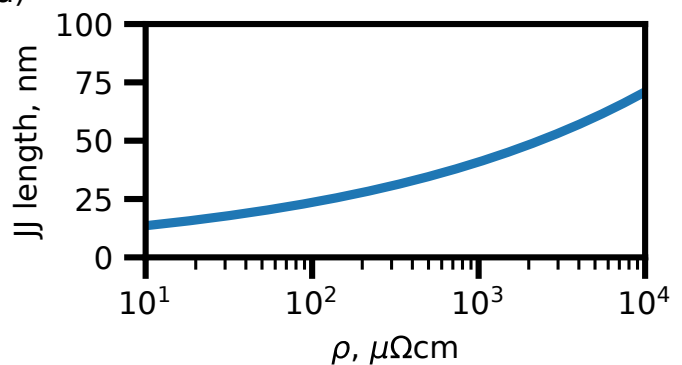

(b)

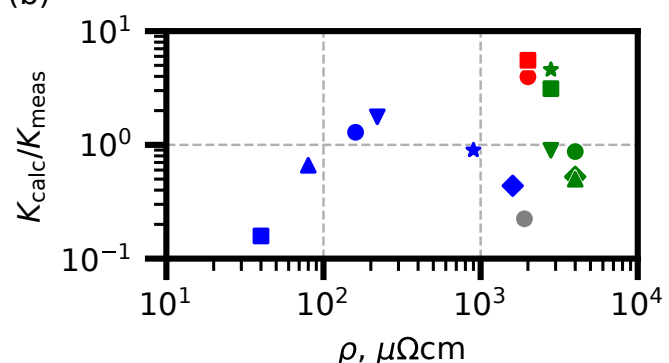

Figure 5.3: (a) Effective Josephson junction length versus grAl film resistivity obtained assuming data reported in Fig. 5.2. (b) Comparison of Kerr coefficients predicted by the 1D JJA model with the JJ size correction and experimental data shown in $\left[\mathrm{MGK}^{+} 18\right]$. Markers correspond to the ones used in the article. Grey circle corresponds to the grAl transmon reported in $\left[\mathrm{WBG}^{+} 20\right]$

realizations differ only by a numerical factor of an order one, see $\left.\left[\mathrm{MGK}^{+} 18\right]\right)$. Assuming all inductance and nonlinearity of the resonator arises from the grAl strip, the nonlinear part of the Kerr Hamiltonian is

$$
H=-\frac{E_{J}}{24 N_{\mathrm{JJ}}^{3}} \varphi^{4}
$$

where $E_{J}$ is the Josephson energy of the effective model JJs, $N_{\mathrm{JJ}}$ is their number, and $\varphi=\phi / \varphi_{0}$ is the phase drop on a single junction (assuming homogeneous phase distribution over the array and absence of phase slips). Since $\varphi=\varphi_{\mathrm{ZPF}}\left(a+a^{+}\right)=\sqrt{\frac{Z_{\text {res }}}{2 R_{\mathrm{q}}}}\left(a+a^{+}\right)=\sqrt{\frac{L_{\mathrm{res}} \omega_{\mathrm{res}}}{2 R_{\mathrm{q}}}}\left(a+a^{+}\right)$with $R_{\mathrm{q}}=\frac{\hbar}{(2 e)^{2}}$, the final equation for the Kerr coefficient is

$$
K=-\frac{E_{\mathrm{J}}}{4 \hbar N_{\mathrm{JJ}}}\left(\frac{\omega_{\mathrm{res}} L_{\mathrm{JJ}}}{R_{\mathrm{q}}}\right)^{2} .
$$

In Fig. 5.3(b), the theoretical prediction for the Kerr coefficient (assuming the 1D JJA model with the corrected $\mathrm{JJ}$ size) is compared with the experimental data reported in $\left[\mathrm{MGK}^{+} 18\right]$. As can be seen, the $\mathrm{JJ}$ size correction allows for a better fit.

However, this model is not absolutely exact. Its prediction for the nonlinearity of the grAl transmon discussed in Ref. $\left[\mathrm{WBG}^{+} 20\right]$ is a factor of 5 higher than the experimentally obtained one. And since the nonlinearity inference in $\left[\mathrm{WBG}^{+} 20\right]$ rely on measurement of frequency detuning between the 0-1 and 1-2 transitions, the deviation of the theoretical model prediction cannot be explained by an experimental systematic error. The mismatch could be caused by the intrinsic structure of grAl, and one might need to consider a 2D of 3D JJ array as a model of interest to be able to give an exact quantitative prediction of the Kerr coefficient. 
Nevertheless, the 1D JJA model accuracy is sufficient for the GrAPA design purposes, and is hence the model of choice for the current work.

\subsection{Design}

(a)

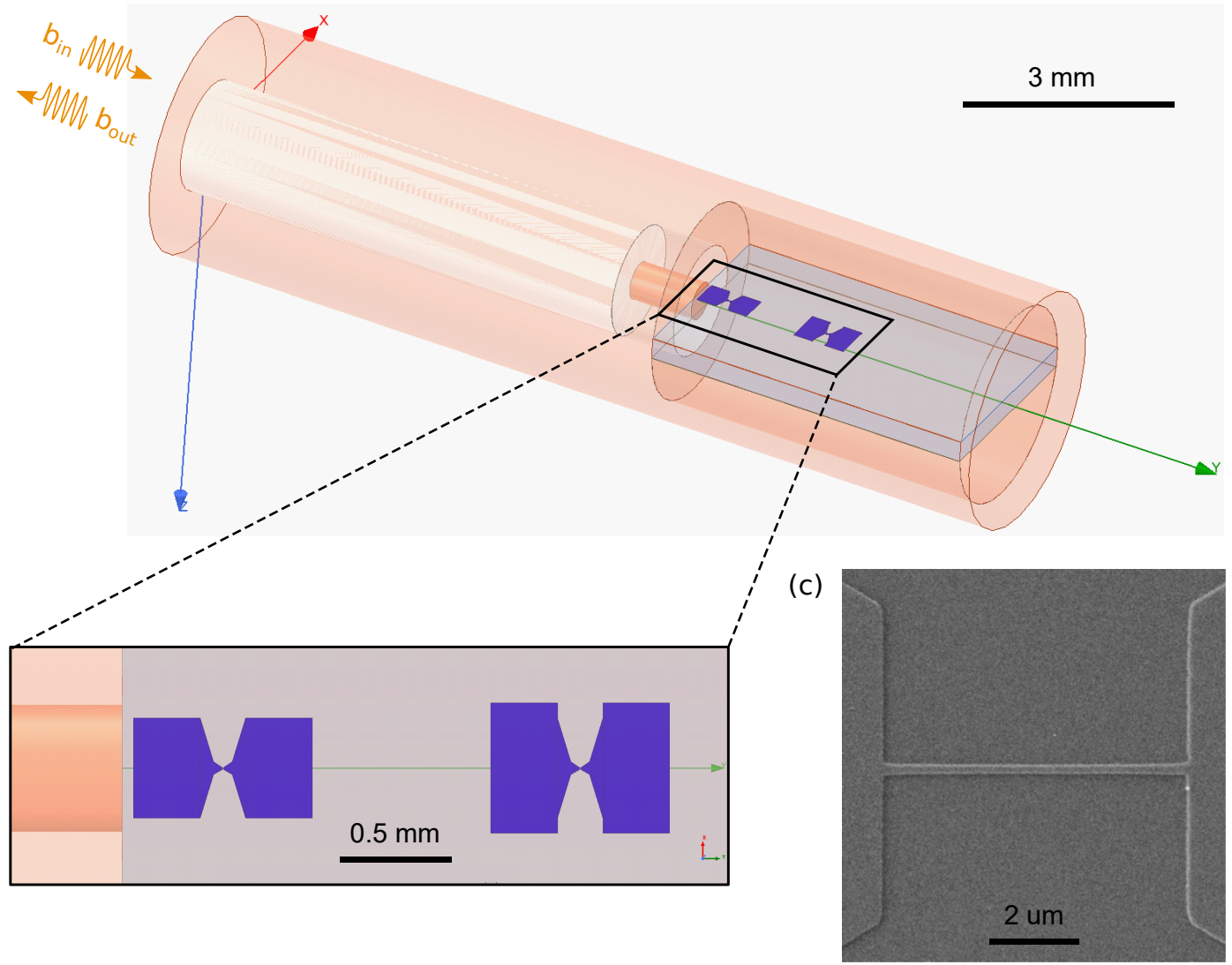

Figure 5.4: $(a, b)$ Images of a GrAPA chip inserted in the waveguide. Capacitor pads of the resonators are shown in blue on top of the grey sapphire wafer. The images were taken from HFSS simulations. (c) SEM image of a grAl strip used as nonlinear inductance of GrAPA.

To achieve signal-pump detuning, a 4-wave-mixing non-degenerate grAl-based amplifier, granular aluminum dimer parametric amplifier (GrADPA), was designed. HFSS model of the GrADPA inserted in a cylindrical waveguide used in [BRW $\left.{ }^{+} 20\right]$ is shown in Fig. 5.4(a). The amplifier consists of two thin-film grAl resonators on top of a C-plane sapphire wafer (see Fig. 5.4(b)). The resonator pads shown in blue are connected by a grAl strip with volume $200 \times 7000 \times 40 \mathrm{~nm}^{3}$, a typical SEM image of which is presented in Fig. 5.4(c). Both pads and strip are deposited in a single normal-angle evaporation to avoid high-power losses appearing due to otherwise inevitable interface between materials with different kinetic inductances (see Appendix A.3.2 for details). The inductance of the resonators is dominated by the grAl kinetic inductance, and the participation ratio of the strip compared to the pads is $p=L_{\text {strip }}^{\mathrm{kin}} / L_{\mathrm{total}} \approx 0.85$.

Similar to the series-capacitor implementation of DJJAAs discussed in chapter 3, one resonator (left) is coupled to the transmission line more than one order of magnitude stronger than the other resonator. This is achieved thanks to the capacitive coupling strength being strongly dependent on the distance from the pin edge, changing from $Q_{\mathrm{c}}=40$ to $Q_{\mathrm{c}}=20000$ as distance between the pin edge and the left edge of a 
resonator in Fig. 5.4(b) is varied between 10 and $2000 \mu \mathrm{m}$. The corresponding HFSS simulation is presented in Fig.5.5(b). The design is wirebonds-free, similar to $\left[\mathrm{NSH}^{+} 14\right]$, which is also beneficial for high magnetic field applications.
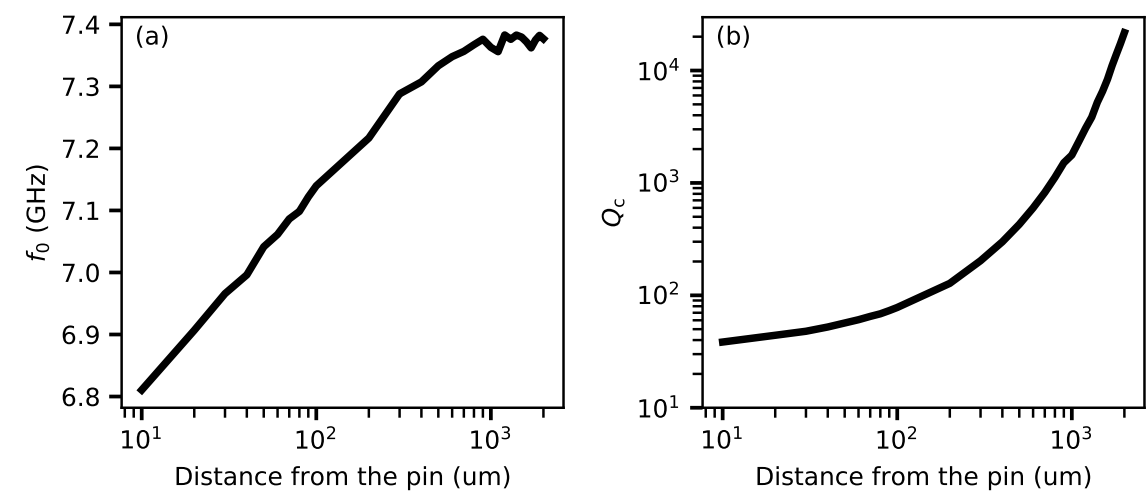

Figure 5.5: $Q_{\mathrm{c}}$ and $f_{0}$ of a single resonator as a function of distance between the pin edge and the closest capacitor pad.

The dipole interaction between resonators creates the modes coupling, enabling non-degenerate amplification regime. To reach this regime, one needs to achieve appropriate hybridization by ensuring that bare eigenfrequencies of the resonators differ by less than few percent (see Fig. 5.6). There are two factors contributing to the imperfection: extra capacitance of the left resonator caused by vicinity of the pin, and mismatch in kinetic inductances of the strips arising due to inhomogeneity in the grAl evaporation process. The capacitors are balanced by adjusting their widths. The evaporation imperfections can be addressed by improving the fabrication procedure or post-fabrication corrections of the second resonator's pads width (e.g. by reactive ion etching $\left.\left[\mathrm{LCC}^{+} 17\right]\right)$.

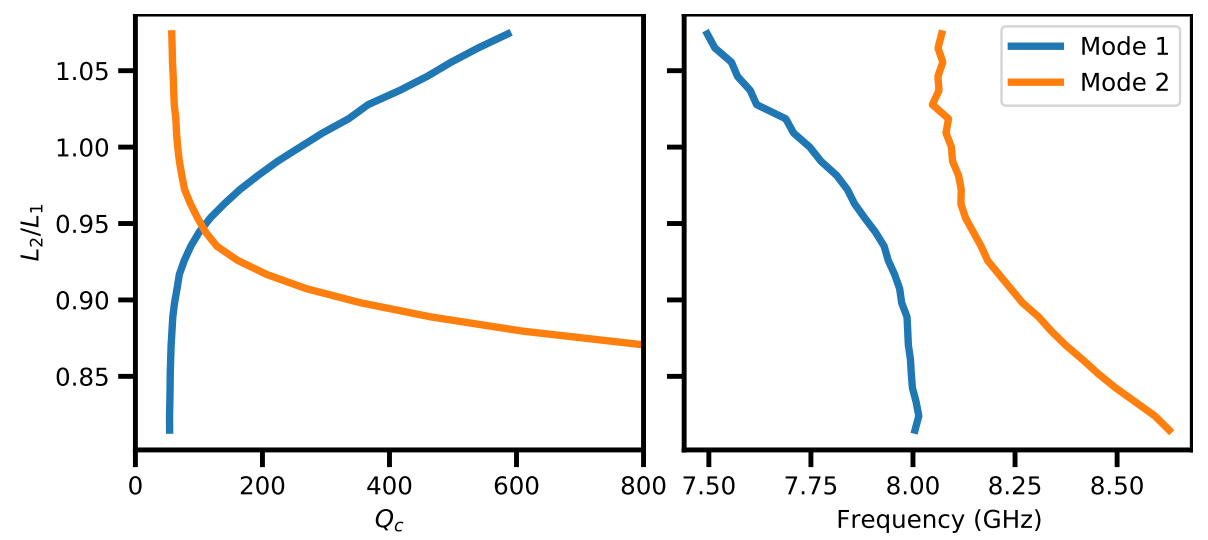

Figure 5.6: Results of HFSS simulation. Quality factor (left plot) and resonance frequency (right plot) of the system's modes for different ratios of grAl strip inductances. 


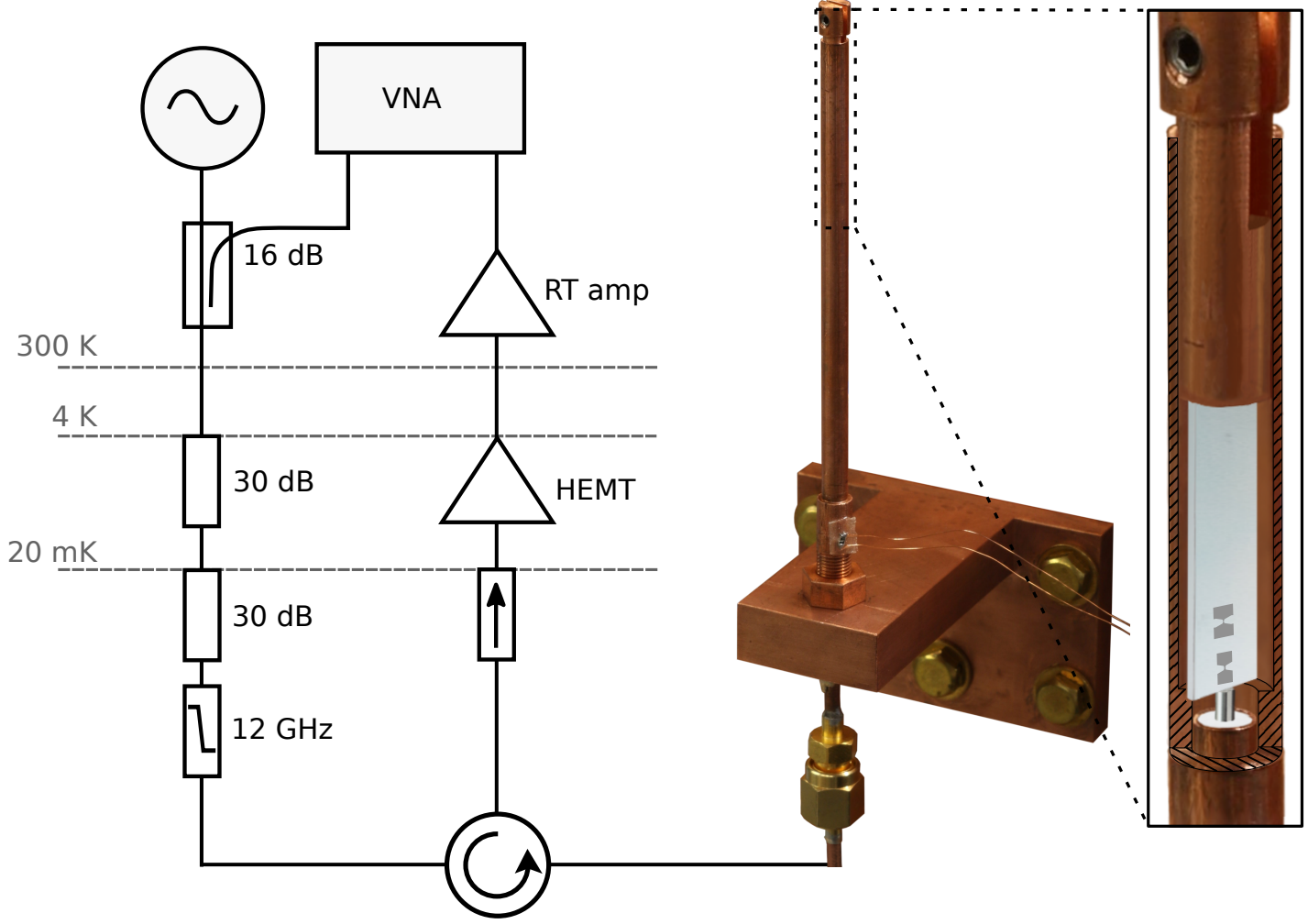

Figure 5.7: Schematic of the experimental setup. The displayed microwave components are thermalized to the nearest temperature stage indicated above them. The right image of the cylindrical waveguide was provided by authors of Ref. [BRW +20$]$. Wires connected to the waveguide are used to apply a DC pulse for a thermal reset and do not affect magnetic field measurements.

\subsection{Experimental results}

Schematic of the measurement setup is shown in Fig. 5.7. The waveguide with the chip is attached to a dilution refrigerator Sionludi [Sio12] with a base temperature of $20 \mathrm{mK}$. Owing to its diameter of $3.6 \mathrm{~mm}$, the waveguide is placed in a coil assembly with 3D field control creating in-plane fields up to $1.2 \mathrm{~T}$. The waveguide pin can be manually moved along the $\mathrm{z}$ axis to adjust coupling strength.

First, to evaluate the resonators' $Q_{i}$, a chip with a single resonator was measured in a dedicated cooldown without applying magnetic fields. The distance between the waveguide pin and the chip edge was adjusted such that $Q_{\mathrm{c}} \sim Q_{\mathrm{i}}$ to minimize influence of Fano effect [Fan61] present due to the finite circulator's isolation [Rie22]. The reflection measured at low power $(\bar{n} \ll 1)$ is shown in Fig. 5.8. Applying the circlefit procedure $\left[\mathrm{PSB}^{+} 15\right]$ yields $Q_{\mathrm{c}}=1.7 \cdot 10^{4}$ and $Q_{\mathrm{i}}=8.8 \cdot 10^{4}$. Since design values of the coupling quality factor are $Q_{\mathrm{c}}^{\text {target }} \sim 10-100$, it is safe to assume that $Q_{\mathrm{i}} / Q_{\mathrm{c}}^{\text {target }} \geq 10^{2}$, which ensures that resonator intrinsic losses are negligible for parametric amplification purposes.

Fig. 5.9 shows a low-power signal reflection from the sample of interest. There are two modes $\left(f_{0}^{\text {left }}=\right.$ $8.47 \mathrm{GHz}, f_{0}^{\text {right }}=8.98 \mathrm{GHz}$ ) with coupling strengths corresponding to $Q_{\mathrm{c}}^{\text {left }}=280, Q_{\mathrm{c}}^{\text {right }}=67$, and internal quality factors $Q_{\mathrm{i}} \sim 10^{4}$. The fact that $Q_{\mathrm{c}}^{\text {left }} \not \approx Q_{\mathrm{c}}^{\text {right }}$ is due to imperfect hybridization mainly caused by difference in the grAl strips resistivities. The obtained modes splitting and coupling strengths correspond to $L_{2} / L_{1} \approx 1.03$ in Fig. 5.6, despite the room-temperature resistance measurements $\left(R_{\text {left }}^{\text {strip }}=8 \mathrm{k} \Omega\right.$, $R_{\text {left }}^{\text {strip }}=8.8 \mathrm{k} \Omega$ ) yielding $L_{2} / L_{1}=1.1$, most probably due to contact pin adjustment accuracy. Ripples 

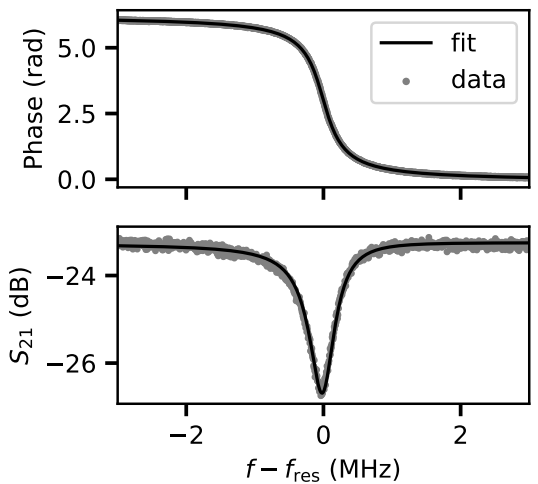

Figure 5.8: Reflection of a weak signal from a decoupled single-resonator GrAPA to extract $Q_{\mathrm{i}}$ : phase (top image) and amplitude (bottom image) versus probe frequency.
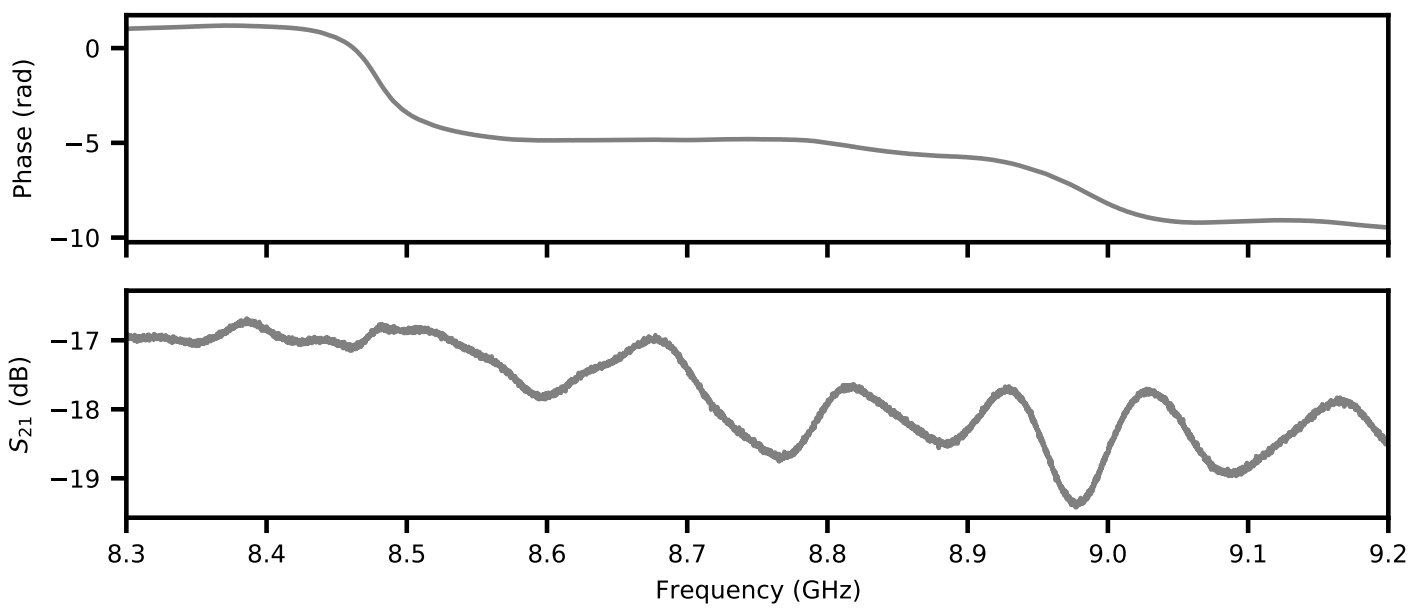

Figure 5.9: Phase (top image) and amplitude (bottom image) of weak signal reflected from the GrADPA versus the probe signal frequency.

in the amplitude response in Fig. 5.9 are present mainly because of the previously discussed Fano effect, which was confirmed by repeating the experiment with a circulator with higher isolation (the corresponding models are LNF-CIC4_12A and QCY-100400).

The reflection power dependence is shown in Fig. 5.10. Notably, there are no losses appearing as drive power increases up to and above the bifurcation point, as is evident from Fig. 5.10(c,d). Corresponding Kerr coefficients (see Fig.5.11) of the modes are $K_{\text {left }} / 2 \pi=-1.6 \mathrm{kHz}, K_{\text {right }} / 2 \pi=-0.8 \mathrm{kHz}$, extracted assuming uncalibrated attenuation between the VNA and the sample is equal to $85 \mathrm{~dB}$. Within the attenuation estimate accuracy of $\pm 10 \mathrm{~dB}$, obtained Kerr coefficients are in agreement with the expected $K_{\text {calc }} \approx 7 \mathrm{kHz}$ calculated following results of Sec. 5.2. 

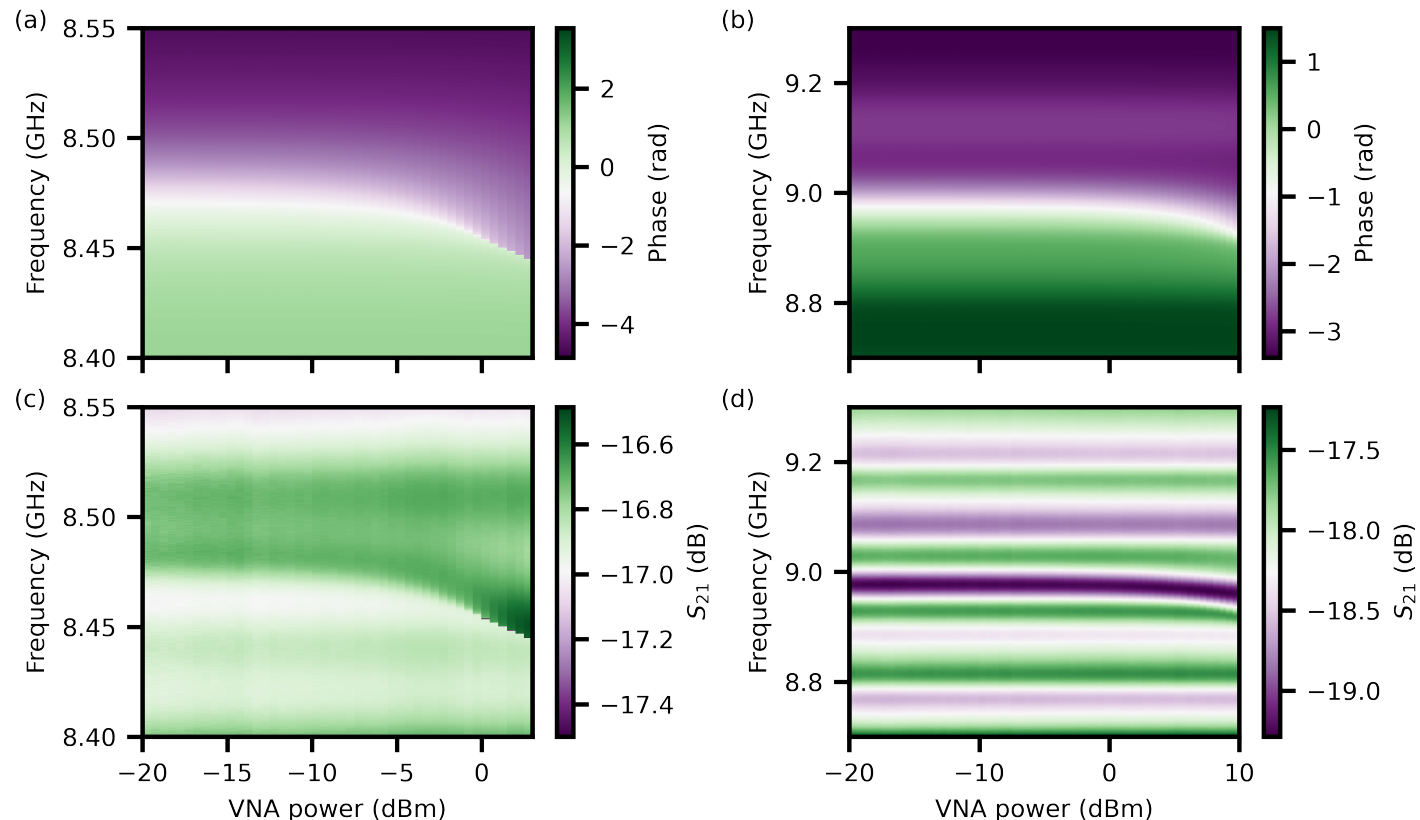

Figure 5.10: (a),(b) Reflection phase and (c),(d) measured amplitude versus different VNA power and frequency. Left (a),(c) and right (b,d) panels correspond to left and right modes, respectively.

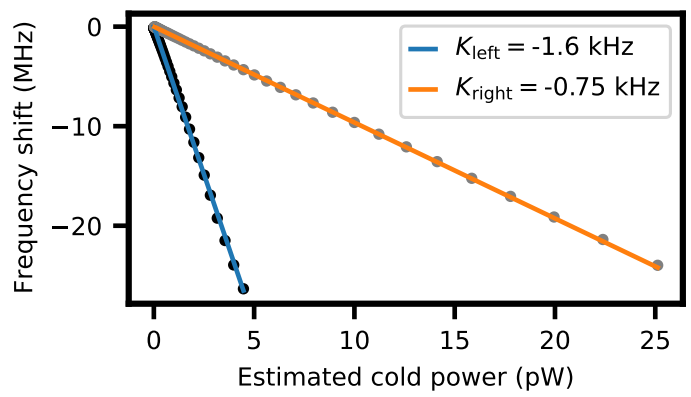

Figure 5.11: Kerr shift for left (black markers) and right (grey markers) modes versus estimated cold power.

\subsubsection{Magnetic field resilience}

Because of the hybridization imperfection, the maximum achievable non-degenerate gain was limited to just a few $\mathrm{dB}$ (see Sec.3.1.1). Leaving this issue for future work, in the frame of this thesis I focus on demonstrating degenerate gain regime in magnetic fields up to $1 \mathrm{~T}$. Throughout the rest of the section, only the left mode is discussed. It was chosen due to having less pronounced ripples (see Fig. 5.9(b)) thus ensuring that any possible impedance-mismatching effects $\left[\mathrm{MWB}^{+} 14\right]$ are minimized.

First, the low-power resonator response was measured in different in-plane fields. As $B_{\text {parr }}$ is increased, the resonance frequency decreases (see Fig. 5.12(a)) because the superconducting gap field dependence obeying [Tin04]

$$
\Delta(B)=\Delta_{0} \sqrt{\frac{1-\left(B / B_{\mathrm{c}}\right)^{2}}{1+\left(B / B_{\mathrm{c}}\right)^{2}}}
$$



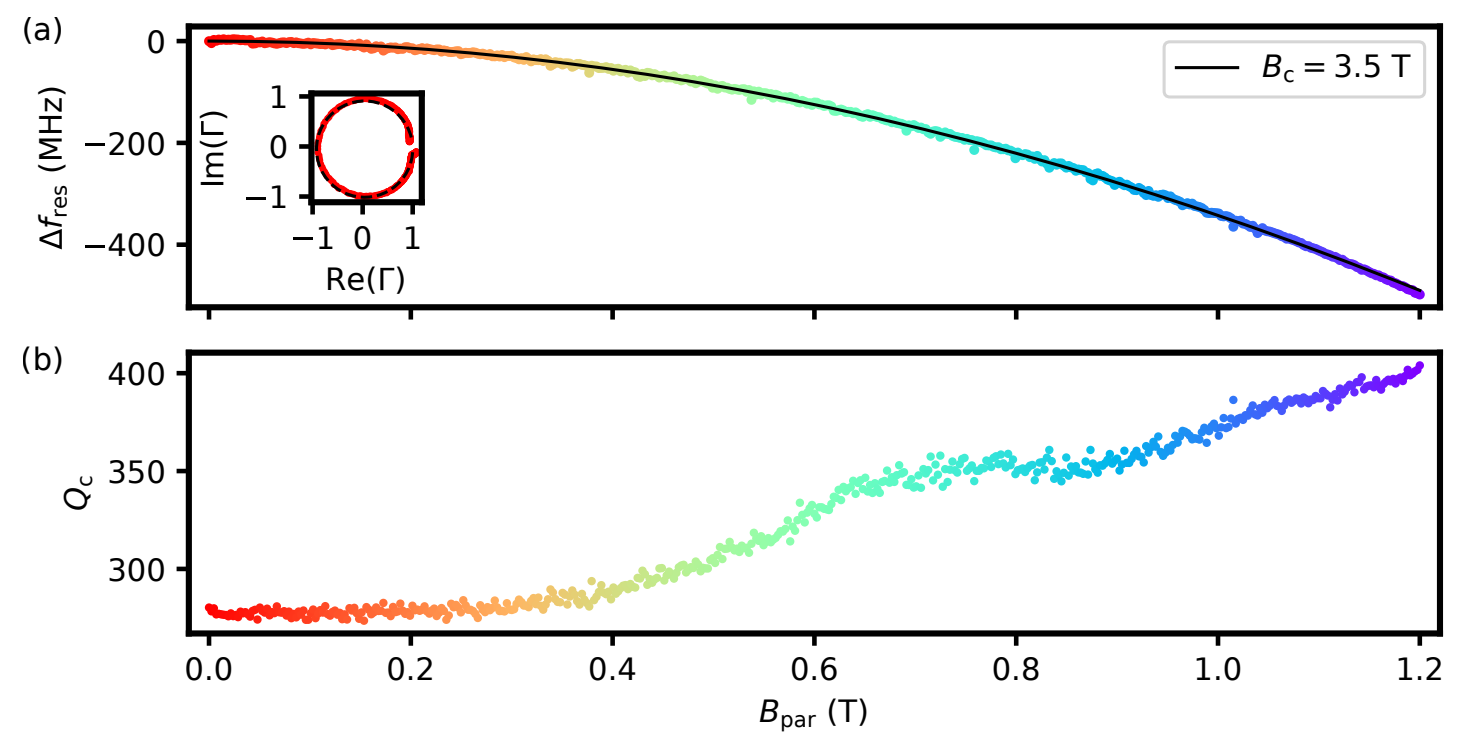

Figure 5.12: (a) Left mode's resonance frequency shift from $f_{0}(B=0)=8.473 \mathrm{GHz}$ versus the in-plane magnetic field $B_{\text {parr }}$. Black line indicates a fit applied to extract critical magnetic field $B_{\mathrm{c}}=3.5 \mathrm{~T}$. The inset plot shows a circle fit (black dotted line) applied for the resonator for $B_{\text {parr }}=0$. (b) $Q_{\mathrm{c}}$ as a function of $B_{\text {parr }}$.

affects the kinetic inductance according to Eq.(5.1). By fitting the frequency shift dependence, the grAl critical field $B_{\mathrm{c}}=3.5 \mathrm{~T}$ was extracted, consistent with previously reported measurements [ACC66; WQL14; $\left.\mathrm{BRW}^{+} 20\right]$. Because grAl is sensitive to perpendicular magnetic field $\left[\mathrm{BRW}^{+} 20\right]$, the unwanted perpendicular component arising due to $0.7^{\circ}$ misalignment of the sample was compensated by applying compensation perpendicular magnetic field $B_{\text {perp }}^{\text {comp }} \sim \mathrm{mT}$ (see Appendix A.3.1 for details). The low-loss condition $Q_{\mathrm{i}} \gg Q_{\mathrm{c}}$ is fulfilled within the measured range of in-plane magnetic field, with $Q_{\mathrm{c}} \sim 10^{2}$ (see Fig. 5.12(b)) and $Q_{\mathrm{i}} \gtrsim 10^{4}$.

\subsubsection{Operating GrADPA in magnetic field}

Applying a pump tone to the sample results in parametric amplification. In Fig. 5.13, examples of averaged degenerate gain profiles are shown for different in-plane fields, up to $0.96 \mathrm{~T}$. The maximum of gain was adjusted to be close to $20 \mathrm{~dB}$, and the measured instantaneous bandwidth was $6.5 \pm 3 \mathrm{MHz}$.

The visible deviation from Lorentzian fit is likely caused by mechanical vibrations. Indeed, for $B_{\text {parr }}=1 \mathrm{~T}$, a $0.1^{\circ}$ tilt results in $B_{\text {perp }}=1 \mathrm{mT}$, shifting resonance frequency by few $\mathrm{MHz}\left[\mathrm{BRW}^{+} 20\right]$. When the pump tone is applied, the gain profile is sensitive to resonance frequency fluctuations, as it changes pump frequency detuning $\omega_{\mathrm{p}}-\omega_{0}$ (see Eq. (2.61)). For the measured sample operated at $G_{0} \approx 20 \mathrm{~dB}$ with optimally chosen pump parameters, $100 \mathrm{kHz}$ resonance frequency shift results in gain deviations of about $1 \mathrm{~dB}$. During the measurement, the gain maximum $G_{0}$ was observed to oscillate by a few $\mathrm{dB}$ with a $\mathrm{Hz}$ frequency, and oscillations were more pronounced at higher magnetic fields.

Due to sensitivity to vibrations, saturation power could be only estimated. In Fig. 5.14, the maximum of gain inferred from a Lorentzian fit of averaged data is shown versus the VNA power for different in-plane fields. Assuming that the estimated attenuation between the sample and the VNA is $85 \pm 10 \mathrm{~dB}$, the measured $1 \mathrm{~dB}$ compression point for $G_{0}=18-22 \mathrm{~dB}$ was -110 :- $100 \mathrm{dBm}$, which is in agreement with the inferred Kerr coefficient. The saturation power can be further increased by decreasing the grAl nonlinearity, which can be achieved by adjusting the volume of the grAl strip. This increase approach, however, can be utilized 

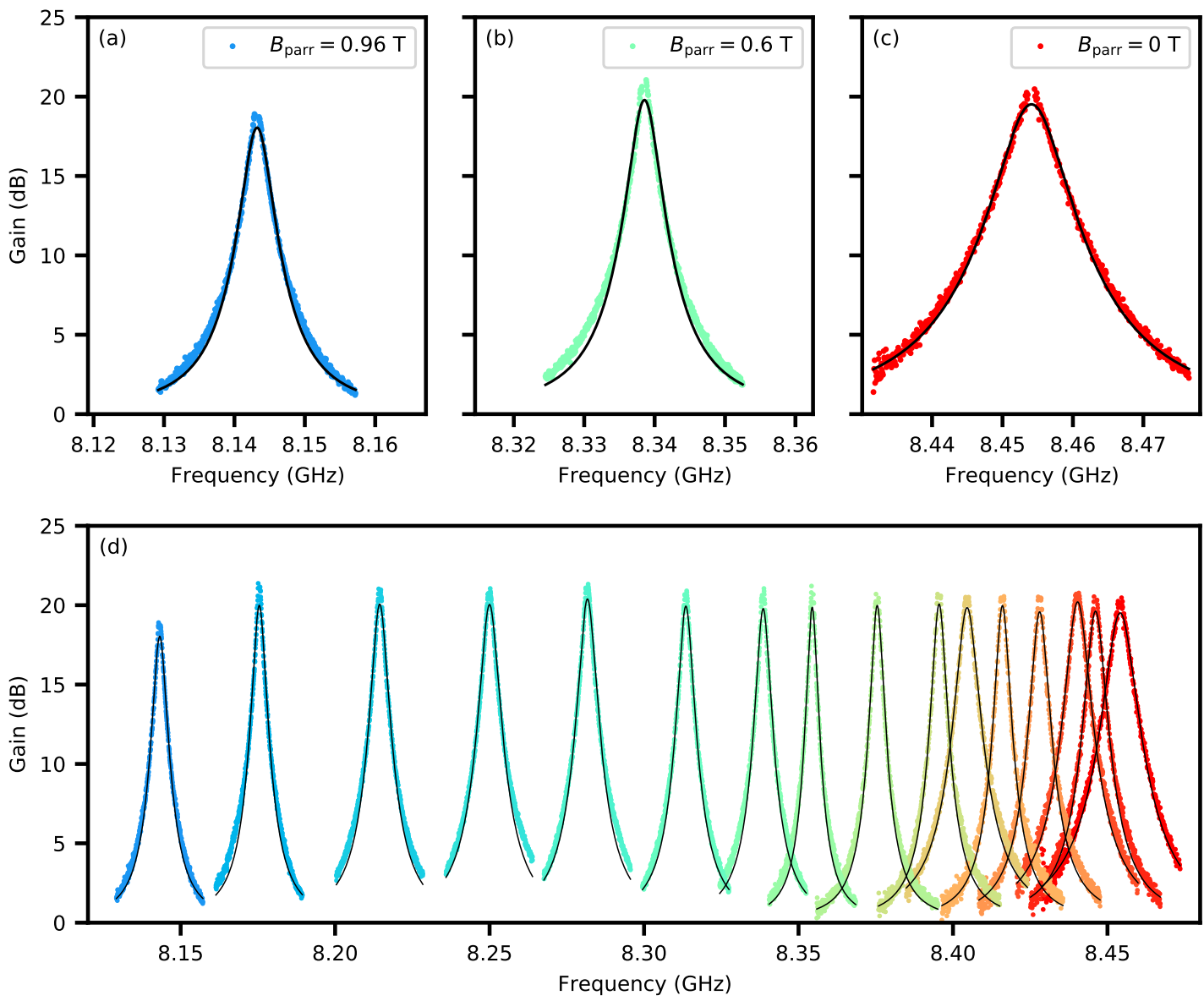

Figure 5.13: Examples of power gain measured for different in-plane fields. Color of the measured points indicates the value of $B_{\text {parr }}$ similar to Fig. 5.12. Black solid curves indicate Lorentzian fits of the corresponding gain profiles.

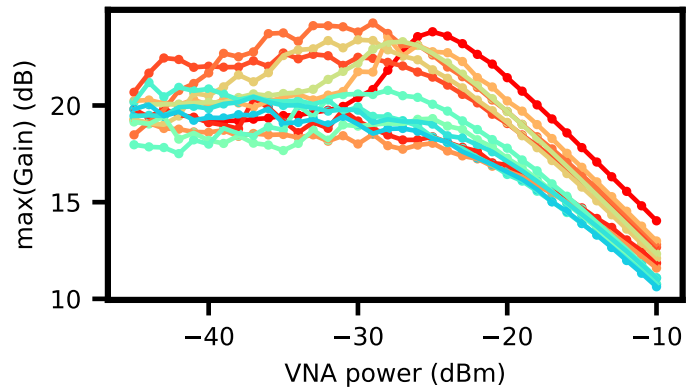

Figure 5.14: Examples of saturation curves (maximum of gain inferred from a Lorentzian fit versus signal power) measured for different in-plane fields. Color of the measured points indicates the value of $B_{\text {parr }}$, similar to Fig. 5.12.

only up to a certain limit. The limit is set by the pump power dissipation in the attenuators, which has to be smaller than the cooling power of the dilution refrigerator. Additionally, the $1 \mathrm{~dB}$ compression point can be increased by reducing the signal-induced Stark shift $\left[\mathrm{PDM}^{+} 19\right]$. This can be realized by utilizing grAl-based Kerr-free elements, such as SNAILs [FSL ${ }^{+}$18] or rf SQUIDs [Zor19]. 


\section{Conclusion and outlook}

This thesis is dedicated to the minimization of the time required to infer the state of an artificial atom. In this work, it was achieved thanks to utilization of a quantum-limited dimer Josephson junction array amplifier with high saturation power, and the fact that the readout of a granular aluminum fuxonium artificial atom remained quantum-non-demolition at relatively large photon numbers in the readout resonator. By exploiting the increasing SNR with $\bar{n}$, a decrease of the artificial atom's state detection time was demonstrated $\left[\mathrm{TWF}^{+} 21\right]$. This was achieved by monitoring the transient trajectories in the IQ plane of the readout resonator response, prompted by quantum jumps between the three lowest energy states of the artificial atom. To infer the qubit state encoded in the readout resonator dynamics, a recursive Bayesian filter was used. Owing to the achieved dynamical regime, the artificial atom's states could be discriminated before the resonator response reaches its steady state.

In this work, the Bayesian filter is applied in post-processing. However, one can imagine using more sophisticated filtering, and potentially running it in real time by utilizing FPGA-based instruments. This can open a way to use the measurement of quantum jumps as a real-life detection tool, informing on the interactions between the quantum system and the environment, such as quasiparticles $\left[\mathrm{RBT}^{+} 13\right.$; $\mathrm{VPS}^{+} 14$; $\left.\mathrm{SDH}^{+} 19\right]$. By deliberately designing a qubit which is strongly coupled to the system of interest, one could even manipulate this system by implementing feedback loops.

As $\bar{n}$ is increased, the state discrimination time decreases, and the limiting factors are the emergence of non-QND processes and nonlinearities of the readout resonator, both intrinsic and inherited. The latter issue can be resolved (or at least minimized) by according optimization of the qubit-resonator system. With this, understanding and mitigating non-QND processes during readout with increasing photons numbers, which is a currently ongoing research, could further increase the quantum state detection speed in both pulsed and continuous measurements.

To enable quantum-limited measurements, a non-degenerate 4-wave-mixing parametric amplifier was developed [WTR ${ }^{+}$20]. The amplifier is based on dispersion-engineered Josephson junction arrays, and is referred to as dimer Josephson junction array amplifier (DJJAA). Thanks to the Josephson-junction-array-based design, the amplifier's saturation power exceeds thousand photons per $\mu s$. The dispersion engineering allows to operate the amplifier in the non-degenerate regime, ensuring few hundreds of $\mathrm{MHz}$ of frequency detuning between pump and signal tones. The amplifier was fabricated with a widely-accessible two-step optical lithography, making it user-friendly in both operation and fabrication. The measured quantum efficiency of a readout chain utilizing an optical-lithography DJJAA was $\eta=60 \pm 10 \%$, on-par with the state-of-the-art Josephson parametric amplifiers. The DJJAA design can be further improved by implementing more sophisticated dispersion engineering schemes to make the amplifier less sensitive to magnetic fields and fabrication inhomogenities, as well as to achieve a control of modes splitting for each individual dimer.

Finally, in this thesis I present the first realization of a parametric amplifier resilient to in-plane magnetic fields up to $1 \mathrm{~T}$. The amplifier is made of two thin-film granular aluminum ( $\mathrm{grAl}$ ) coupled resonators formed by capacitor pads connected with a strip. The corresponding modes' Kerr coefficients were adjusted to be in the $\mathrm{kHz}$ range. Only one of the resonators is coupled to a transmission line, and the system's modes form a dimer, similar to the DJJAA's dimers, thus enabling non-degenerate regime. Although the presented 
realization of a granular aluminum dimer parametric amplifier (GrADPA) does not have any loops, the non-degenerate gain profile can be tuned within few hundreds of $\mathrm{MHz}$ owing to the vast range of pump parameters which can be used to obtain a gain profile with the maximum of gain $G_{0}=20 \mathrm{~dB}$.

The GrADPA was characterized in magnetic fields up to $1.2 \mathrm{~T}$. No significant increase of internal losses was observed, indicating that amplifier's added noise does not change in the in-plane field. Degenerate gain was measured up to $1 \mathrm{~T}$. Unfortunately, non-degenerate regime with $G_{0}=20 \mathrm{~dB}$ was not achievable due to inhomogeneity of the grAl film resistivity. On top of that, the amplifier performance was limited due to vibrations of the waveguide which were leading to oscillations of perpendicular component of the magnetic field.

The future work includes minimization of the waveguide vibrations to the acceptable level. The inhomogeneity of the grAl film resistivity can be addressed by utilizing post-fabrication adjustment of the resonators's frequencies by reducing the capacitor pads' width by using etching or evaporating metal with laser. By utilizing grAl gradiometric SNAILs, one could make a flux-tunable 3-wave mixing GrAPA. 


\section{A Appendix}

\section{A.1 DJJAA}

\section{A.1.1 Capacitance and inductance matrices}

Eqs. (A.1),(A.2), introduce capacitance and inductance matrices of a Josephson junction array (see Fig. 3.5(a)), galvanically connected with edges to ground plane and/or transmission line(s). For the dimerized JJA (see Fig. 3.6(a)), the middle parts of the same matrices which are "responsible" for dimerization are presented in Eqs. (A.3),(A.4).

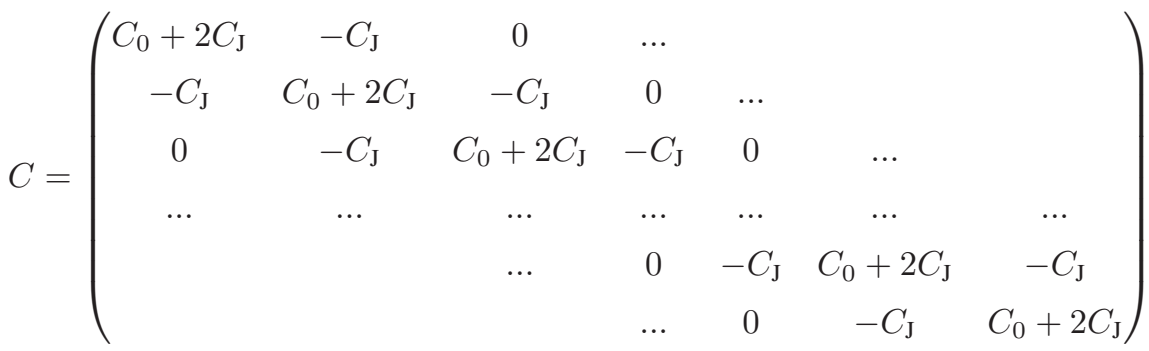

$$
\begin{aligned}
& L^{-1}=\left(\begin{array}{ccccccc}
\frac{2}{L_{\mathrm{J}}} & \frac{1}{L_{\mathrm{J}}} & 0 & \cdots & & & \\
-\frac{1}{L_{\mathrm{J}}} & \frac{2}{L_{\mathrm{J}}} & -\frac{1}{L_{\mathrm{J}} \mathrm{J}} & 0 & \cdots & & \\
0 & -\frac{1}{L_{\mathrm{J}}} & \frac{2}{L_{\mathrm{J}}} & -\frac{1}{L_{\mathrm{J}}} & 0 & \cdots & \\
\cdots & \cdots & \cdots & \cdots & \cdots & \cdots & \ldots \\
& & \cdots & 0 & -\frac{1}{L_{\mathrm{J}}} & \frac{2}{L_{\mathrm{J}}} & -\frac{1}{L_{\mathrm{J}}} \\
& & & \cdots & 0 & -\frac{1}{L_{\mathrm{J}}} & \frac{2}{L_{\mathrm{J}}}
\end{array}\right) \\
& C_{\text {dimer }}=\left(\begin{array}{ccccccccc}
\ldots & \ldots & \ldots & \ldots & \ldots & \ldots & & & \\
\ldots & -C_{\mathrm{J}} & C_{0}+2 C_{\mathrm{J}} & -C_{\mathrm{J}} & 0 & \ldots & & & \\
\ldots & 0 & -C_{\mathrm{J}} & C_{\mathrm{c}} & -C_{\mathrm{c}} & 0 & \ldots & & \\
& \ldots & 0 & C_{\mathrm{c}} & -C_{\mathrm{c}} & -C_{\mathrm{J}} & 0 & \ldots & \\
& & \ldots & 0 & -C_{\mathrm{J}} & C_{0}+2 C_{\mathrm{J}} & -C_{\mathrm{J}} & 0 & \ldots \\
& & \ldots & \ldots & \ldots & \ldots & \ldots & \ldots & \ldots
\end{array}\right) \\
& L_{\text {dimer }}^{-1}=\left(\begin{array}{ccccccccc}
\cdots & \ldots & \ldots & \ldots & \ldots & \ldots & & & \\
\cdots & -\frac{1}{L_{\mathrm{J}}} & \frac{2}{L_{\mathrm{J}}} & -\frac{1}{L_{\mathrm{J}}} & 0 & \ldots & & & \\
\cdots & 0 & -\frac{1}{L_{\mathrm{J}}} & \frac{1}{L_{\mathrm{J}}} & 0 & \ldots & & & \\
& \ldots & 0 & 0 & \frac{1}{L_{\mathrm{J}}} & -\frac{1}{L_{\mathrm{J}}} & 0 & \ldots & \\
& & \ldots & 0 & -\frac{1}{L_{\mathrm{J}}} & \frac{2}{L_{\mathrm{J}}} & -\frac{1}{L_{\mathrm{J}}} & 0 & \ldots \\
& & \ldots & \ldots & \ldots & \ldots & \ldots & \ldots & \ldots
\end{array}\right)
\end{aligned}
$$




\section{A.1.2 E-beam-lithography DJJAA}

(a)

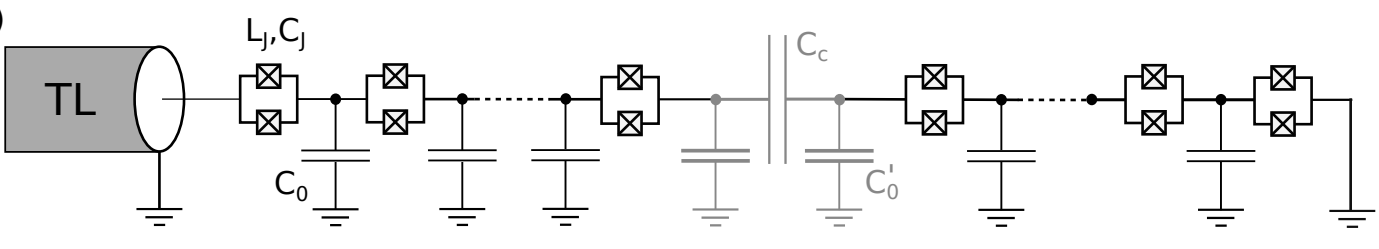

(b)

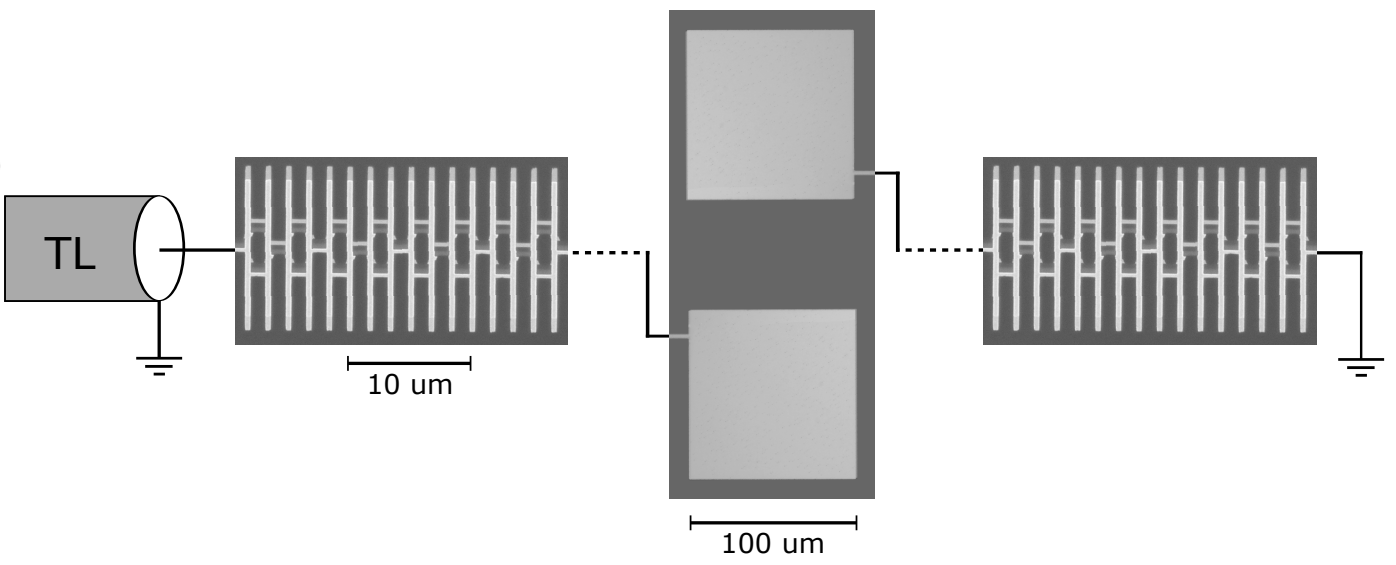

Figure A.1: (a) Schematic of the DJJAA. (b) SEM images of the SQUID array and the series capacitor.

Fig. A.1 shows SEM images of the DJJAA, as well as the equivalent schematic. The amplifier is made of an array of 100 SQUIDs fabricated on a 300 um thick sapphire double-side polished wafer by utilizing a bridge-free technique [LPP $\left.{ }^{+} 11\right]$. The SQUID array is interrupted in the middle with a series capacitor. Both ground plane and coupling capacitor pads are separated from the SQUID arrays by a $200 \mathrm{~nm}$ wide wire, which strongly reduces $\left[\mathrm{PDM}^{+} 19\right]$ effects of flux trapping and Meissner currents. To ensure uniformity of the ground capacitancies and to minimize losses, a $200 \mathrm{~nm}$ thick gold layer was deposited on the backside of the chip. The effective model (Fig. A.1(a)) circuit parameters of the DJJAA are: $C_{c}=6.5 \mathrm{fF}, C_{c}^{\prime}=20 \mathrm{fF}$, $C_{0}=0.5 \mathrm{fF}, C_{\mathrm{J}}=360 \mathrm{fF}, L_{J}=240 \mathrm{pH}$.
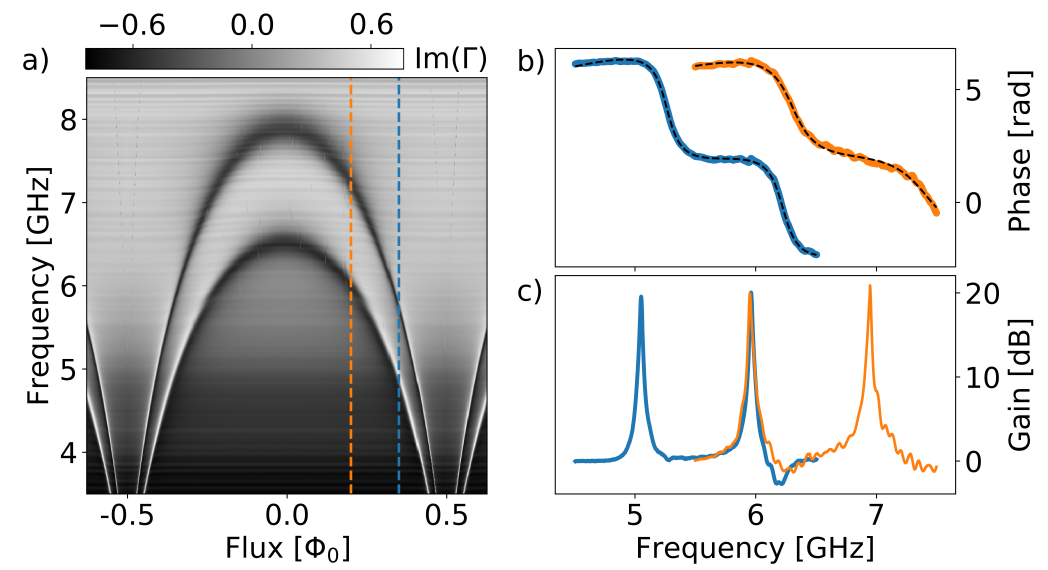

Figure A.2: (a) Imaginary part of weak reflected signal versus frequency and external magnetic flux. (b) Phase of the weak reflected signal shown versus frequency for two flux biases indicated in panel (a) with dashed lines of corresponding colors. (c) Non-degenerate gain profiles obtained for flux biases indicated in panel (a). 
To characterize the DJJAA, we used the experimental setup shown in Fig. 3.9(a). The qubit sample in front of the DJJAA is the transmon qubit discussed in Sec. 3.4.1.

In Fig. A.2(a), phase response of the weak signal is shown for two selected flux biases. The corresponding Bose-Hubbard model parameters extracted with a circle fit procedure $\left[\mathrm{PSB}^{+} 15\right]$ are listed in Table A.1. In Fig. A.2(c), examples of non-degenerate gain profiles with the maximum gain of $20 \mathrm{~dB}$ and instantaneous bandwidth of $20-30 \mathrm{MHz}$ are presented for the corresponding flux biases. Wiggles visible in the gain curves are present most likely due to impedance mismatches $\left[\mathrm{MWB}^{+} 14\right]$, while the difference in the maximum value of the Lorentzian peaks is caused by intrinsic losses $\gamma / \kappa \approx 0.1$.

Table A.1: List of linear Bose-Hubbard parameters extracted from weak reflection probe measurements shown in Fig. A.2.

\begin{tabular}{|c|c|c|c|c|c|}
\hline$\Phi / \Phi_{0}$ & $\omega_{1}, \mathrm{GHz}$ & $\omega_{\mathrm{r}}, \mathrm{GHz}$ & $J, \mathrm{GHz}$ & $\kappa, \mathrm{GHz}$ & $\gamma, \mathrm{GHz}$ \\
\hline 0.2 & 6.966 & 6.786 & 0.749 & 0.59 & 0.05 \\
\hline 0.35 & 5.763 & 5.726 & 0.391 & 0.49 & 0.035 \\
\hline
\end{tabular}
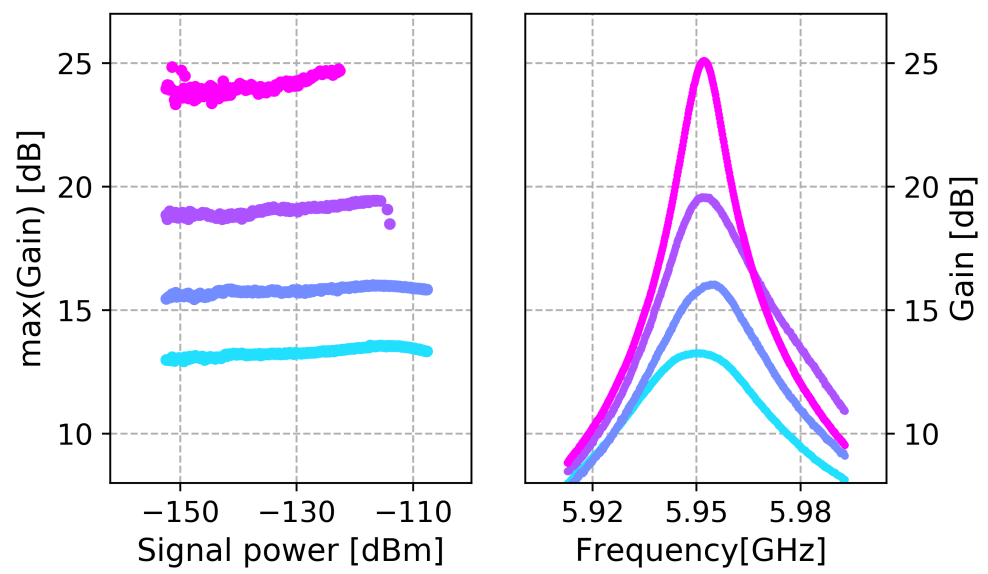

Figure A.3: (a) Maximum of gain versus calibrated signal power. (b) Gain profiles at maximum achieved signal powers.

In Fig. A.3(a), maximum of the left lobe of the gain profile for $\Phi=0.35 \Phi_{0}$ is shown versus signal power. Curves have an abrupt drop, most probably caused by the DJJAA pump response entering the bifurcation area due to either flux noise or AC Stark frequency shift caused by signal photons. Because of that, it was impossible for this device to measure the $1 \mathrm{~dB}$ compression point. However, the amplifier was provided with $20 \mathrm{~dB}$ of non-degenerate gain up to $P_{\text {sig }} \approx-120 \mathrm{dBm}$, coresponding to 250 photons $/ \mu \mathrm{s}$.

\section{A.1.3 Elastic scattering on a two-level system}

Here, an elastic scattering on a two-level system is discussed for the case of transmission measurement. The modelled system is a two-level system coupled to two baths corresponding to left and right propagating waves. The coupling strength $\kappa$ is equal for both modes, and is the qubit's dominant loss mechanism. The signal is assumed to be sent (and read out) via right propagating waves. 
By utilizing the input-output formalism, the transmission coefficient can be written as

$$
t=\beta_{\text {out }}(t) / \beta_{\text {in }}(t)=1+\sqrt{\kappa}\left\langle\sigma_{x}\right\rangle / \beta_{\text {in }}(t),
$$

where $\beta_{\text {in/out }}$ is the input/otput field of the right-propagating bath. To obtain the expression for $\left\langle\sigma_{x}\right\rangle$, the density matrix evolution needs to be calculated. It is described by

$$
\begin{array}{r}
\dot{\rho}=-\frac{i}{2} \omega_{\mathrm{q}}\left[\sigma_{\mathrm{z}}, \rho\right]+\sqrt{\kappa}\left(e^{i \omega_{\mathrm{dr}} t} \beta_{\mathrm{in}}^{*}[\sigma, \rho]-e^{-i \omega_{\mathrm{dr}} t} \beta_{\mathrm{in}}\left[\sigma^{+}, \rho\right]+\kappa\left(2 \sigma \rho \sigma^{+}-\sigma^{+} \sigma \rho-\rho \sigma^{+} \sigma\right)=\right. \\
=i \omega_{\mathrm{q}}\left(\begin{array}{cc}
0 & -\rho_{01} \\
\rho_{10} & 0
\end{array}\right)+\sqrt{\kappa} \beta_{\mathrm{in}}\left[e^{i \omega_{\mathrm{dr}} t}\left(\begin{array}{cc}
-\rho_{01} & -0 \\
\rho_{00}-\rho_{11} & \rho_{01}
\end{array}\right)+e^{-i \omega_{\mathrm{dr}} t}\left(\begin{array}{cc}
-\rho_{10} & \rho_{00}-\rho 11 \\
0 & \rho_{10}
\end{array}\right)\right]+ \\
+\kappa\left(\begin{array}{rr}
-2 \rho_{00} & -\rho_{01} \\
\rho_{10} & 2 \rho_{00}
\end{array}\right),
\end{array}
$$

where $\omega_{\mathrm{q}} / 2 \pi$ is the $0-1$ transition frequency of the qubit, and $\omega_{\mathrm{dr}} / 2 \pi$ is the drive frequency. Note that the Lindblad term is doubled due to the fact that the qubit is coupled to two baths. By definition, the relaxation and dephasing rates are the coefficients in the Lindblad term, so for the current model ( assuming qubit has no intrinsic losses) $\Gamma=2 \gamma=2 \kappa$. Here, $\Gamma$ and $\gamma$ are the $T_{1}$ and $T_{2}$ decay rates.

The density matrix evolution can be rewritten as:

$$
\begin{array}{r}
\dot{\rho}_{00}=-\sqrt{\kappa} \beta_{\text {in }}\left(e^{-i \omega_{\mathrm{dr}} t} \rho_{10}+e^{i \omega_{\mathrm{dr}} t} \rho_{01}\right)-2 \kappa \rho_{00} \\
\dot{\rho}_{01}=\left(-i \delta \omega_{\mathrm{q}}-\kappa\right) \rho_{01}+\sqrt{\kappa} \beta_{\mathrm{in}} e^{-i \omega_{\mathrm{dr}} t}\left(\rho_{00}-\rho_{11}\right) \\
\dot{\rho}_{10}=\left(i \delta \omega_{\mathrm{q}}-\kappa\right) \rho_{10}+\sqrt{\kappa} \beta_{\mathrm{in}} e^{i \omega_{\mathrm{dr}} t}\left(\rho_{00}-\rho_{11}\right)
\end{array}
$$

Since interaction between the qubit and propagating waves is $\sigma_{x}$, one can write that:

$$
\dot{\rho}=\left(\begin{array}{cc}
0 & -i \omega_{\mathrm{dr}} \rho_{01} \\
i \omega_{\mathrm{dr}} \rho_{10} & 0
\end{array}\right)
$$

By introducing new variables

$$
S^{+}=\rho_{01} e^{i \omega_{\mathrm{dr}} t} ; S=\rho_{10} e^{-i \omega_{\mathrm{dr}} t} ; \delta w=\omega_{\mathrm{dr}}-\omega_{\mathrm{q}},
$$

the density matrix evolution can be rewritten as $(\operatorname{Tr} \rho=1)$ :

$$
\begin{array}{r}
0=-\sqrt{\kappa} \beta_{\text {in }}\left(S+S^{+}\right)-2 \kappa \rho_{00} \\
0=\left(-i \delta \omega_{\mathrm{q}}-\kappa\right) S^{+}+\sqrt{\kappa} \beta_{\text {in }}\left(2 \rho_{00}-1\right) \\
0=\left(i \delta \omega_{\mathrm{q}}-\kappa\right) S+\sqrt{\kappa} \beta_{\text {in }}\left(2 \rho_{00}-1\right) .
\end{array}
$$

This system of equations yields

$$
\begin{aligned}
S^{+} & =\sqrt{\kappa} \beta_{\text {in }} \frac{-i \delta w-\kappa}{\delta w^{2}+\kappa^{2}+2 \kappa \beta_{\mathrm{in}}^{2}} \\
S & =\sqrt{\kappa} \beta_{\text {in }} \frac{i \delta w-\kappa}{\delta w^{2}+\kappa^{2}+2 \kappa \beta_{\mathrm{in}}^{2}}
\end{aligned}
$$


and

$$
\left\langle\sigma_{x}\right\rangle=\operatorname{Tr}\left(\rho \sigma_{x}\right)=\rho_{01}+\rho_{10}=S^{+} e^{-i \omega_{\mathrm{dr}} t}+S e^{i \omega_{\mathrm{dr}} t} .
$$

By utilizing RWA and leaving only terms with $e^{-i \omega_{\mathrm{dr}} t}$, the final expression can be obtained $(\kappa=\gamma)$ :

$$
t=1-\frac{(1+i \delta w / \gamma)}{1+(\delta w / \gamma)^{2}+2 \Gamma \beta_{\mathrm{in}}^{2} / \Gamma \gamma},
$$

with the Rabi frequency

$$
\Omega^{2}=\frac{2 \Gamma P_{\mathrm{in}}}{\hbar \omega_{\mathrm{q}}}
$$

\section{A.2 High-power quantum jumps detection}

\section{A.2.1 Fluxonium parameters}

Table A.2 lists parameters of the system consisting of the fluxonium artificial atom and the readout resonator utilized in chapter 4 .

Table A.2: List of the qubit-resonator circuit parameters. The Josephson energy $E_{\mathrm{J}}$ is in-situ flux-tunable via the SQUID junction.

\begin{tabular}{|c|c|}
\hline \multicolumn{2}{|c|}{ Fluxonium } \\
\hline$E_{C} / h$ & $2.8 \mathrm{GHz}$ \\
\hline$E_{L} / h$ & $0.71 \mathrm{GHz}$ \\
\hline$E_{\mathrm{J}} / h, \mathrm{GHz}$ & $7.1 \mathrm{GHz}$ \\
\hline Flux bias & $0.542 \Phi_{0}$ \\
\hline$f_{\text {ge }}$ & $902 \mathrm{MHz}$ \\
\hline$f_{\text {ef }}$ & $7.701 \mathrm{GHz}$ \\
\hline$T_{q}$ & $31 \mathrm{mK}$ \\
\hline$T_{1}$ & $32.1 \pm 0.7 \mu \mathrm{s}$ \\
\hline$T_{2}$ & $0.73 \pm 0.06 \mu \mathrm{s}$ \\
\hline Readout resonator \\
\hline$\kappa / 2 \pi$ & $1.1 \mathrm{MHz}$ \\
\hline$\gamma / 2 \pi$ & $20 \mathrm{kHz}$ \\
\hline$f_{\text {res }}$ & $7.247 \mathrm{GHz}$ \\
\hline$K_{\text {intrinsic }} / 2 \pi$ & $-2.4 \mathrm{kHz}$ \\
\hline \multicolumn{2}{|c|}{ Couplings } \\
\hline$\chi_{\mathrm{ge}} / 2 \pi$ & $-1.09 \mathrm{MHz}$ \\
\hline$\chi_{\mathrm{ef}} / 2 \pi$ & $1.46 \mathrm{MHz}$ \\
\hline \multicolumn{2}{|c}{} \\
\hline
\end{tabular}

\section{A.2.2 Example of gain obtained on the fluxonium readout resonator}

Fig. A.4 shows an example of power gain of the granular aluminum readout resonator measured with a VNA. Power gain was obtained thanks to the intrinsic nonlinearity of the resonator $K_{\text {intrinsic }} / 2 \pi=-2.4 \mathrm{kHz}$. The 
fluxonium artificial atom was flux-tuned away from the flux point used for other discussed experiments and had a negligible contribution to the presented response.

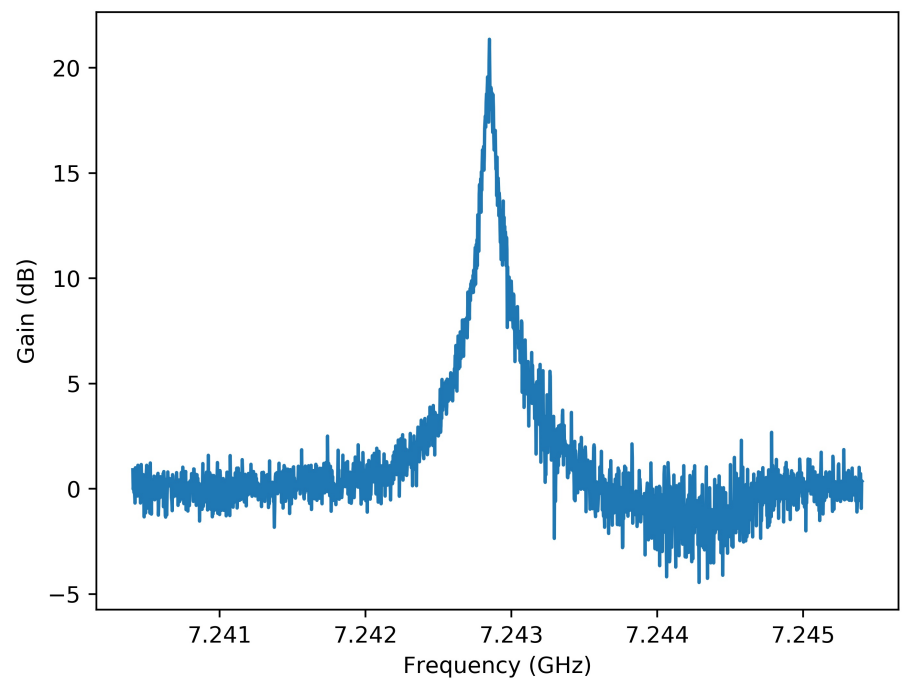

Figure A.4: Example of a power gain obtained when pumping the fluxonium readout resonator.

\section{A.2.3 Measurement setup}

The measurement setup used for qubit readout discussed in chapter 4 is shown in Fig. A.5. To generate readout pulses, the output of the Arbitrary Wave Generator (AWG) with the carrier frequency IF $=62.5 \mathrm{MHz}$ is upconverted on an IQ mixer by utilizing a tone of a local oscillator applied at frequency $f_{\text {res }}-$ IF. The upconverted tone is then split into two parts on a power divider. One part, denoted reference, is downconverted to the IF frequency and recorded on the ADC. In front of the ADC input channels, passband filters with a band of $10 \mathrm{MHz}$ were introduced to get rid of unwanted noise and harmonics.

The other part of the split upconverted tone, referred to as signal, is sent through the $30 \mathrm{mK}$ dillution refrigerator. Before the signal reaches the qubit sample, it passes through several commercial attenuators, $8 \mathrm{GHz}$ low-pass commercial filter, and home-made infrared filter. These elements are introduced to ensure that noise of the signal at the input of the qubit sample is as close to quantum noise as possible. After reflecting from the readout resonator, the signal is amplified by the DJJAA operated in a non-degenerate regime with $G\left(f_{\text {res }}\right)=20 \mathrm{~dB}$. To apply the pump tone via a separate input line, a $20 \mathrm{~dB}$ directional coupler was used.

After the DJJAA, the signal is amplified by a High Electron Mobility Transistor (HEMT) amplifier and room-temperature amplifiers, and is downconverted and recorded on ADC. An additional post-processing filtering with a band of $4 \mathrm{MHz}$ was applied to the signal trace. To extract $\mathrm{Q}$ and I quadratures, reference and signal data recorded by ADC is multiplied sample-wise, with respective zero and quarter-period delays. Prior to multiplication, signal and reference data are aligned to account for an $80 \mathrm{~ns}$ delay caused by the time required for signal to travel through the cryostat. 


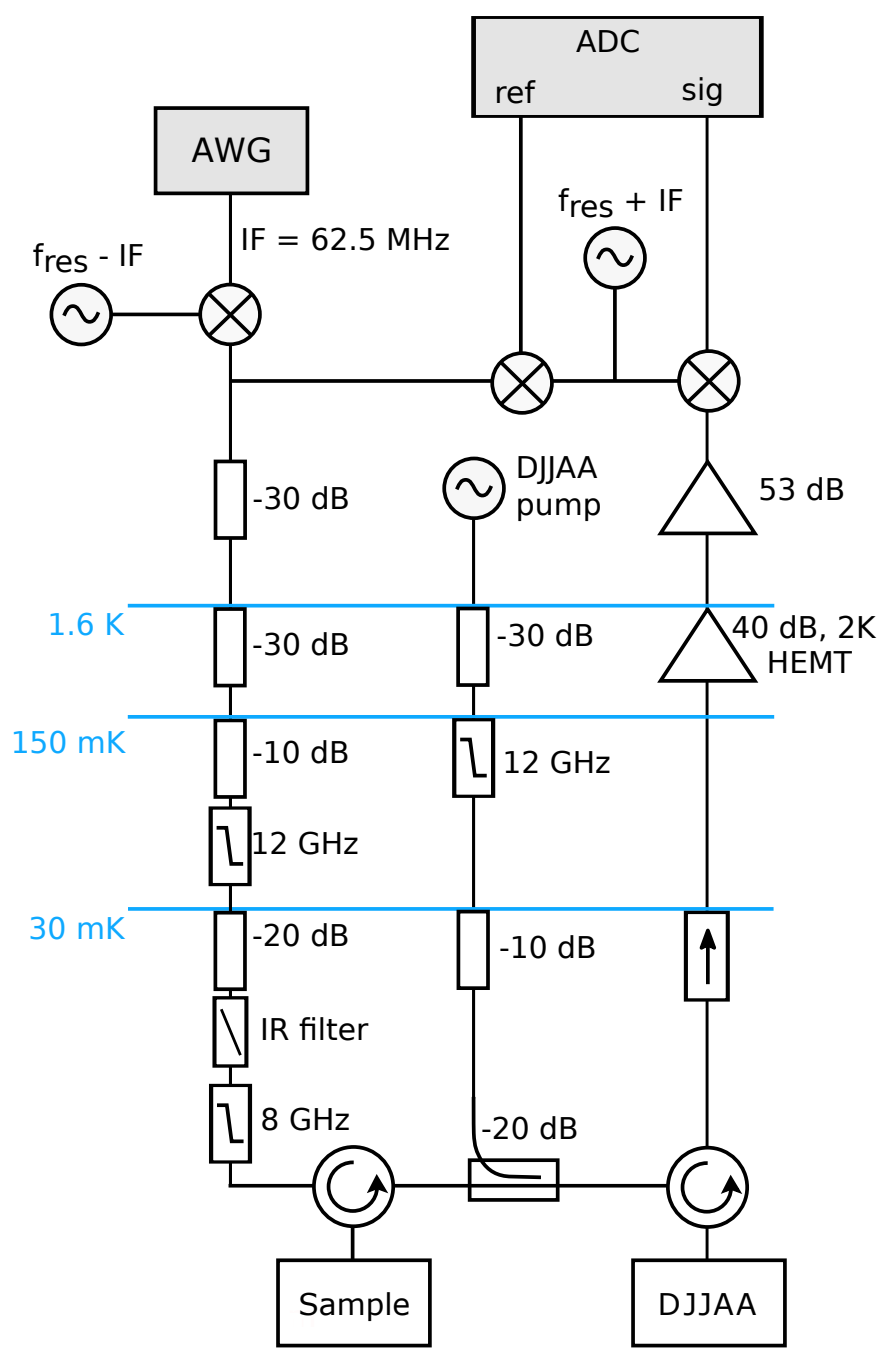

Figure A.5: Schematics of the measurement setup. The displayed microwave components are thermalized to the nearest temperature stage indicated above them.

\section{A.3 GrAPA}

\section{A.3.1 Compensation of the perpendicular field}

While grAl is resilient to in-plane fields up to few Tesla, the critical field for the perpendicular field is lower by two orders of magnitude. GrAPA resonance frequency is thus sensitive to perpendicular component of magnetic field, typically caused by a minor chip tilt with respect to the Helmholtz coil. To compensate this unwanted component, a cancellation field was applied.

In Fig. A.6, the compensation field is shown for different in-plane fields. For each point, the applied perpendicular field was adjusted by maximizing resonance frequency of the GrAPA. Black line indicates linear fit in the 0-1 $\mathrm{T}$ in-plane field range. 


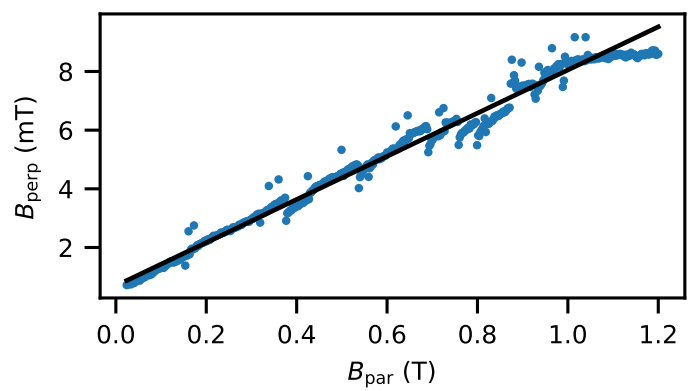

Figure A.6: Compensation perpendicular magnetic field applied to negate effect of chip misalignment.

\section{A.3.2 High-power issues at the interface between materials with different kinetic inductance}

In this section, another way of making the grAl-based amplifier is discussed. For this approach, the amplifier capacitor pads are made of another metal, such as $\mathrm{Al}$ or $\mathrm{Nb}$. The design's benefits are a more easily achievable coupling to the transmission line (increasing the pads area does not change participation ratio of the nonlinear inductance) and an enhanced critical magnetic field of the pads due to utilization of $\mathrm{Nb}$.

In Fig. A.7, images of this realization of GrAPA are shown. The aluminum capacitor pads visible in Fig. A.7(a) have a fractal structure which allows to maximize their magnetic critical field. Figs. A.7(b,c) demonstrate connection between Al electrodes (vertical) and the grAl strip (horizontal) with volume $V_{\mathrm{grAl}}=$ $4.8 \cdot 0.2 \cdot 0.04 \mathrm{\mu m}^{3}$ and normal-state resistivity $\rho_{\mathrm{n}}=1000 \pm 500 \mathrm{\mu} \Omega \mathrm{cm}$. The amplifier is fabricated on a C-plane sapphire wafer in a single-step lithography by utilizing a three-angle shadow evaporation, similar to Ref. $\left[\mathrm{WBG}^{+} 20\right]$.

(a)



(b)

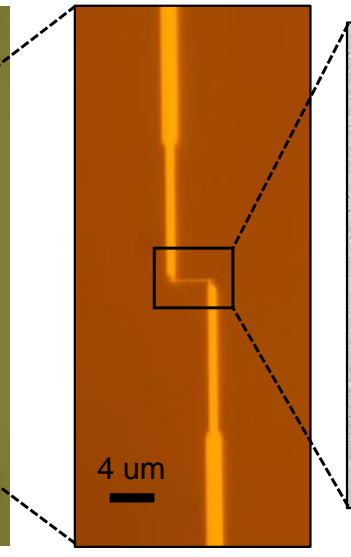

(c)

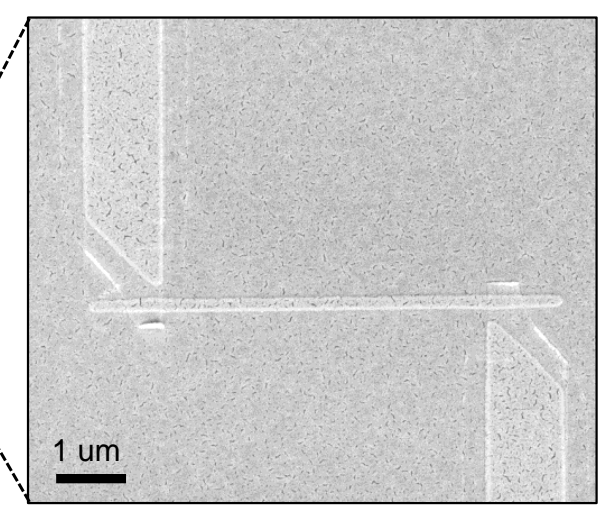

Figure A.7: (a),(b) Optical microscope images of a GrAPA, consisting of two fractalized Al pads connected with a grAl strip. (c) SEM image of a grAl strip (horisontal) connected with two Al electrodes(vertical). Volume of the grAl in-between electrodes is $V_{\text {grAl }}=4.8 \cdot 0.2 \cdot 0.04 \mathrm{\mu m}^{3}$, and the normal-state resistivity is $\rho_{\mathrm{n}}=1000 \pm 500 \mu \Omega \mathrm{cm}$. The structure was fabricated in a single lithography step by performing a three-angle evaporation.

Response of the sample to a microwave probe is shown in Fig. A.8 for different drive powers. At low power, the system works as an overcoupled resonator thanks to the fact that $Q_{i} / Q_{c}>10$. However, as the 



Figure A.8: Power dependence of the GrAPA responce measured with VNA: (a) resonance frequency, (b) $Q_{\mathrm{i}} / Q_{\mathrm{c}}$, (c) amplitude of the measured signal as a function of VNA power. Grey dotted line indicates VNA power corresponding to the bifurcation power.
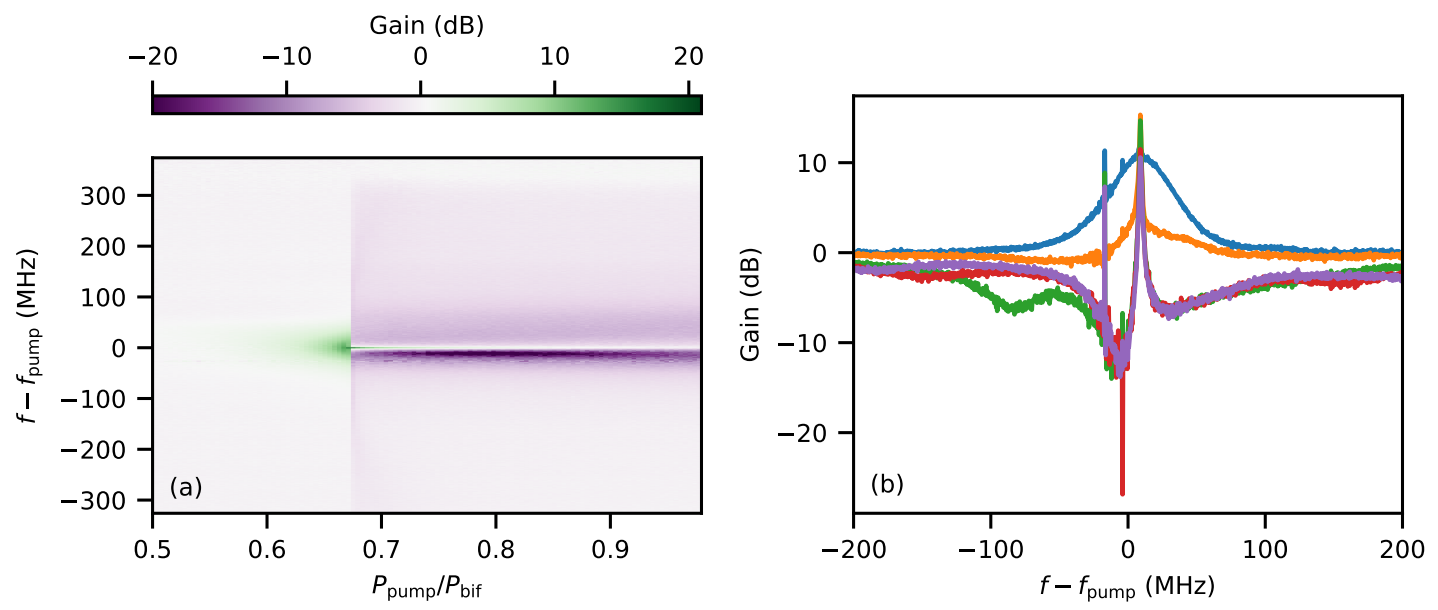

Figure A.9: (a) Gain as a function of pump power and signal frequency. Pump is normalized by the bifurcation drive power, and frequency is shifted by the pump frequency $f_{\text {pump }}=7.926 \mathrm{GHz}$. (b) Some chosen gain profiles showcasing behaviour of the device influenced by losses.

probe power approaches the bifurcation power, $Q_{i}$ demonstrates a significant decrease. Since parametric amplification requires pump powers slightly below the bifurcation point, the amplifier's gain is in the "lossy" regime (see Sec. 2.3.3), as is evident in Fig. A.9. Because of that, noise added by this device is increased well above the quantum limit. The same behaviour was observed in $\mathrm{Nb} / \mathrm{grAl}$ samples and in grAl/grAl samples with the first layer of grAl having resistivity about ten times lower than the second one.

The onset of the lossy behavior is most likely due to the interface of superconductors with different kinetic inductance. Cooper pairs following the least-action route minimize their path through the high- $L_{\mathrm{kin}}$ metal. As a result, current in the $\mathrm{Al} / \mathrm{grAl}$ interface flows via a small area of the interface, and at some point the current density probably exceeds the critical one. Although not a problem for low-power devices $\left[\mathrm{GSG}^{+} 19 ; \mathrm{WBG}^{+} 20\right]$, the loss rise problem makes combination of superconductors with significantly different kinetic inductances suboptimal for parametric amplification purposes. 



\section{Bibliography}

[AAAe16] B. P. Abbott, R. Abbott, T. D. Abbott, and et.al. Observation of gravitational waves from a binary black hole merger. Phys. Rev. Lett., 116:061102, Feb 2016. URL: https://link.aps.org/doi/10.1103/PhysRevLett.116.061102, doi:10. 1103/PhysRevLett.116.061102.

$\left[\mathrm{AAB}^{+}\right.$19] Frank Arute, Kunal Arya, Ryan Babbush, Dave Bacon, and et. al. Quantum supremacy using a programmable superconducting processor. Nature, 574(7779):505-510, Oct 2019. doi:10.1038/s41586-019-1666-5.

[ACC66] B. Abeles, Roger W. Cohen, and G. W. Cullen. Enhancement of superconductivity in metal films. Phys. Rev. Lett., 17:632-634, Sep 1966. URL: https://link.aps.org/doi/10. 1103/PhysRevLett.17.632, doi:10.1103/PhysRevLett.17.632.

[Agr13] G. P. Agrawal. Nonlinear Fiber Optics. Elsevier Academic Press, 2013.

[AT55] S. H. Autler and C. H. Townes. Stark effect in rapidly varying fields. Phys. Rev., 100:703722, Oct 1955. URL: https://link.aps.org/doi/10.1103/PhysRev.100.703, doi: 10.1103/PhysRev.100.703.

[Aum20] Jose Aumentado. Superconducting parametric amplifiers: The state of the art in josephson parametric amplifiers. IEEE Microwave Magazine, 21(8):45-59, 2020. doi : 10 .1109/MMM . 2020.2993476 .

$\left[\mathrm{AZA}^{+} 10\right]$ O. Astafiev, A. M. Zagoskin, A. A. Abdumalikov, Yu. A. Pashkin, T. Yamamoto, K. Inomata, Y. Nakamura, and J. S. Tsai. Resonance fluorescence of a single artificial atom. 327(5967):840-843, 2010. URL: https://science.sciencemag.org/content/327/ 5967/840, doi:10.1126/science.1181918.

[BCMS19] Colin D. Bruzewicz, John Chiaverini, Robert McConnell, and Jeremy M. Sage. Trapped-ion quantum computing: Progress and challenges. Applied Physics Reviews, 6(2):021314, 2019. doi:10.1063/1.5088164.

[BGB09] Maxime Boissonneault, J. M. Gambetta, and Alexandre Blais. Dispersive regime of circuit qed: Photon-dependent qubit dephasing and relaxation rates. Phys. Rev. A, 79:013819, Jan 2009. URL: https://link.aps.org/doi/10.1103/PhysRevA.79.013819, doi: 10.1103/PhysRevA.79.013819.

[BGO20] Alexandre Blais, Steven M. Girvin, and William D. Oliver. Quantum information processing and quantum optics with circuit quantum electrodynamics. Nature Physics, 16(3):247256, 2020. URL: http://dx.doi.org/10.1038/s41567-020-0806-z, doi:10.1038/ s41567-020-0806-z.

[BKP13] Peter Brooks, Alexei Kitaev, and John Preskill. Protected gates for superconducting qubits. Phys. Rev. A, 87:052306, May 2013. URL: https://link.aps.org/doi/10.1103/ PhysRevA.87.052306, doi:10.1103/PhysRevA.87.052306. 
[BKRB08] Jan Benhelm, Gerhard Kirchmair, Christian F. Roos, and Rainer Blatt. Towards faulttolerant quantum computing with trapped ions. Nature Physics, 4(6):463-466, Jun 2008. doi: $10.1038 /$ nphys961.

$\left[\mathrm{BLH}^{+}{ }^{15}\right]$ Nicholas T. Bronn, Yanbing Liu, Jared B. Hertzberg, Antonio D. Córcoles, Andrew A. Houck, Jay M. Gambetta, and Jerry M. Chow. Broadband filters for abatement of spontaneous emission in circuit quantum electrodynamics. Applied Physics Letters, 107(17):172601, Oct 2015. URL: http://dx.doi.org/10.1063/1.4934867, doi:10.1063/1.4934867.

$\left[\mathrm{BPK}^{+} 16\right]$ A. Bienfait, J. J. Pla, Y. Kubo, M. Stern, X. Zhou, C. C. Lo, C. D. Weis, T. Schenkel, M. L. W. Thewalt, D. Vion, D. Esteve, B. Julsgaard, K. Mølmer, J. J. L. Morton, and P. Bertet. Reaching the quantum limit of sensitivity in electron spin resonance. Nature Nanotechnology, 11(3):253-257, Mar 2016. doi:10.1038/nnano.2015.282.

$\left[\mathrm{BRW}^{+} 20\right]$ K. Borisov, D. Rieger, P. Winkel, F. Henriques, F. Valenti, A. Ionita, M. Wessbecher, M. Spiecker, D. Gusenkova, I. M. Pop, and et al. Superconducting granular aluminum resonators resilient to magnetic fields up to 1 tesla. Applied Physics Letters, 117(12):120502, Sep 2020. URL: http://dx . doi .org/10.1063/5.0018012, doi:10.1063/5.0018012.

$\left[\mathrm{BSM}^{+} 10\right]$ N. Bergeal, F. Schackert, M. Metcalfe, R. Vijay, V. E. Manucharyan, L. Frunzio, D. E. Prober, R. J. Schoelkopf, S. M. Girvin, and M. H. Devoret. Phase-preserving amplification near the quantum limit with a josephson ring modulator. Nature, 465(7294):64-68, May 2010. URL: http://dx.doi.org/10.1038/nature09035, doi:10.1038/nature09035.

$\left[\mathrm{BTH}^{+} 18\right]$ C. C. Bultink, B. Tarasinski, N. Haandbæk, S. Poletto, N. Haider, D. J. Michalak, A. Bruno, and L. DiCarlo. General method for extracting the quantum efficiency of dispersive qubit readout in circuit qed. Applied Physics Letters, 112(9):092601, 2018. doi:10.1063/1. 5015954.

$\left[\mathrm{BVB}^{+} 21\right]$ Alexander Bilmes, Serhii Volosheniuk, Jan David Brehm, Alexey V. Ustinov, and Jürgen Lisenfeld. Quantum sensors for microscopic tunneling systems. npj Quantum Information, 7(1), Feb 2021. URL: http://dx.doi.org/10.1038/s41534-020-00359-x, doi: 10 . $1038 / \mathrm{s} 41534-020-00359-\mathrm{x}$.

[BW08] Lapo Bogani and Wolfgang Wernsdorfer. Molecular spintronics using single-molecule magnets. Nature Materials, 7(3):179-186, Mar 2008. doi :10.1038/nmat2133.

[Car02] H. J. Carmichael. The Physics of Superconductors; 2nd ed. Springer, Berlin, 2002.

[Cav82] Carlton M. Caves. Quantum limits on noise in linear amplifiers. Phys. Rev. D, 26:1817-1839, Oct 1982. URL: https://link.aps .org/doi/10.1103/PhysRevD.26.1817, doi:10. 1103/PhysRevD . 26.1817.

[CBL07] M. A. Castellanos-Beltran and K. W. Lehnert. Widely tunable parametric amplifier based on a superconducting quantum interference device array resonator. Applied Physics Letters, 91(8):083509, Aug 2007. URL: http://dx.doi.org/10.1063/1.2773988, doi:10. $1063 / 1.2773988$

$\left[\mathrm{CDG}^{+}\right]$A. A. Clerk, M H Devoret, Steven M. Girvin, Florian Marquardt, and R. J. Schoelkopf. doi:10.1103/RevModPhys.82.1155.

$\left[\mathrm{CSDF}^{+} 21\right]$ Anasua Chatterjee, Paul Stevenson, Silvano De Franceschi, Andrea Morello, Nathalie P. de Leon, and Ferdinand Kuemmeth. Semiconductor qubits in practice. Nature Reviews Physics, 3(3):157-177, Mar 2021. doi:10.1038/s42254-021-00283-9. 
$\left[\mathrm{DCJ}^{+}\right.$07] M. V. Gurudev Dutt, L. Childress, L. Jiang, E. Togan, J. Maze, F. Jelezko, A. S. Zibrov, P. R. Hemmer, and M. D. Lukin. Quantum register based on individual electronic and nuclear spin qubits in diamond. Science, 316(5829):1312-1316, 2007. URL: https://www. science. org/doi/abs/10.1126/science.1139831, doi:10.1126/science.1139831.

[DGGI73] G. Deutscher, M. Gershenson, E. Grünbaum, and Y. Imry. Granular superconducting films. Journal of Vacuum Science and Technology, 10(5):697-701, 1973. doi: $10.1116 / 1.1318416$.

[DiV95] David P. DiVincenzo. Quantum computation. Science, 270(5234):255-261, 1995. URL: https://www.science.org/doi/abs/10.1126/science.270.5234.255, doi: $10.1126 /$ science.270.5234.255.

$\left[\mathrm{DRM}^{+} 20\right]$ R. Dassonneville, T. Ramos, V. Milchakov, L. Planat, É. Dumur, F. Foroughi, J. Puertas, S. Leger, K. Bharadwaj, J. Delaforce, C. Naud, W. Hasch-Guichard, J. J. García-Ripoll, N. Roch, and O. Buisson. Fast high-fidelity quantum nondemolition qubit readout via a nonperturbative cross-kerr coupling. Phys. Rev. X, 10:011045, Feb 2020. URL: https : / / ink. aps.org/doi/10.1103/PhysRevX.10.011045, doi:10.1103/PhysRevX.10.011045.

$\left[\mathrm{EKT}^{+}\right.$19] A. Eddins, J. M. Kreikebaum, D. M. Toyli, E. M. Levenson-Falk, A. Dove, W. P. Livingston, B. A. Levitan, L. C. G. Govia, A. A. Clerk, and I. Siddiqi. High-efficiency measurement of an artificial atom embedded in a parametric amplifier. Phys. Rev. X, 9:011004, Jan 2019. URL: https://link.aps.org/doi/10.1103/PhysRevX.9.011004, doi: 10.1103/PhysRevX.9.011004.

[ERPR21] Martina Esposito, Arpit Ranadive, Luca Planat, and Nicolas Roch. Perspective on traveling wave microwave parametric amplifiers. Applied Physics Letters, 119(12):120501, Sep 2021. URL: http://dx.doi.org/10.1063/5.0064892, doi:10.1063/5.0064892.

$\left[\mathrm{ESM}^{+} 14\right]$ C. Eichler, Y. Salathe, J. Mlynek, S. Schmidt, and A. Wallraff. Quantum-limited amplification and entanglement in coupled nonlinear resonators. Phys. Rev. Lett., 113:110502, Sep 2014. URL: https://link.aps.org/doi/10.1103/PhysRevLett.113.110502, doi:10.1103/PhysRevLett.113.110502.

[EW14] Christopher Eichler and Andreas Wallraff. Controlling the dynamic range of a josephson parametric amplifier. EPJ Quantum Technology, 1(1):2, Jan 2014. doi:10.1140/epjqt2.

[Fan61] U. Fano. Effects of configuration interaction on intensities and phase shifts. Phys. Rev., 124:1866-1878, Dec 1961. URL: https://link.aps.org/doi/10.1103/PhysRev. 124.1866, doi:10.1103/PhysRev.124.1866.

[Fey] Richard P. Feynman. doi:10.1007/BF02650179.

[FLQL16] Wei Feng, Pengfei Liang, Lupei Qin, and Xin-Qi Li. Exact quantum bayesian rule for qubit measurements in circuit qed. Scientific Reports, 6(1):20492, Feb 2016. doi:10.1038/ srep20492.

$\left[\mathrm{FSL}^{+} 18\right]$ N. E. Frattini, V. V. Sivak, A. Lingenfelter, S. Shankar, and M. H. Devoret. Optimizing the nonlinearity and dissipation of a snail parametric amplifier for dynamic range. Physical Review Applied, 10(5), Nov 2018. URL: http://dx.doi .org/10.1103/PhysRevApplied. 10.054020, doi:10.1103/physrevapplied.10.054020. 
[FVS+ 17] N. E. Frattini, U. Vool, S. Shankar, A. Narla, K. M. Sliwa, and M. H. Devoret. 3-wave mixing josephson dipole element. Applied Physics Letters, 110(22):222603, May 2017. URL: http://dx.doi.org/10.1063/1.4984142, doi:10.1063/1.4984142.

$\left[\mathrm{FWB}^{+}\right.$19] Felix Friedrich, Patrick Winkel, Kiril Borisov, Hannes Seeger, Christoph Sürgers, Ioan M Pop, and Wolfgang Wernsdorfer. Onset of phase diffusion in high kinetic inductance granular aluminum micro-squids. Superconductor Science and Technology, 32(12):125008, Oct 2019. URL: http://dx.doi.org/10.1088/1361-6668/ab4918, doi:10.1088/1361-6668/ ab4918.

[GC85] Cw Gardiner and $\mathrm{Mj}$ Collett. Input and output in damped quantum systems: Quantum stochastic differential equations and the master equation. Phys. Rev. A, 31(6):37613774, 1985. URL: http://link.aps.org/doi/10.1103/PhysRevA.31.3761, doi: 10.1103/PhysRevA.31.3761.

[GMDP $\left.{ }^{+} 21\right]$ András Gyenis, Pranav S. Mundada, Agustin Di Paolo, Thomas M. Hazard, Xinyuan You, David I. Schuster, Jens Koch, Alexandre Blais, and Andrew A. Houck. Experimental realization of a protected superconducting circuit derived from the $0-\pi$ qubit. PRX Quantum, 2:010339, Mar 2021. URL: https://link.aps.org/doi/10.1103/PRXQuantum.2. 010339, doi:10.1103/PRXQuantum.2.010339.

$\left[\mathrm{GMF}^{+} 17\right]$ Jay M. Gambetta, Conal E. Murray, Y.-K.-K. Fung, Douglas T. McClure, Oliver Dial, William Shanks, Jeffrey W. Sleight, and Matthias Steffen. Investigating surface loss effects in superconducting transmon qubits. IEEE Transactions on Applied Superconductivity, 27(1):1-5, Jan 2017. URL: http://dx.doi.org/10.1109/TASC.2016.2629670, doi:10.1109/tasc.2016.2629670.

[GMS $\left.{ }^{+} 18\right]$ Lukas Grünhaupt, Nataliya Maleeva, Sebastian T. Skacel, Martino Calvo, Florence LevyBertrand, Alexey V. Ustinov, Hannes Rotzinger, Alessandro Monfardini, Gianluigi Catelani, and Ioan M. Pop. Loss mechanisms and quasiparticle dynamics in superconducting microwave resonators made of thin-film granular aluminum. Phys. Rev. Lett., 121:117001, Sep 2018. URL: https://link.aps.org/doi/10.1103/PhysRevLett.121.117001, doi:10.1103/PhysRevLett.121.117001.

[GSG $\left.{ }^{+} 19\right]$ Lukas Grünhaupt, Martin Spiecker, Daria Gusenkova, Nataliya Maleeva, Sebastian T. Skacel, Ivan Takmakov, Francesco Valenti, Patrick Winkel, Hannes Rotzinger, Alexey V. Ustinov, and Ioan M. Pop. Granular aluminium as a superconducting material for high-impedance quantum circuits. Nature Materials, 18(8):816-819, Apr 2019. URL: http://dx.doi . org/10.1038/s41563-019-0350-3, doi:10.1038/s41563-019-0350-3.

[GSG $\left.{ }^{+} 21\right]$ Daria Gusenkova, Martin Spiecker, Richard Gebauer, Madita Willsch, Dennis Willsch, Francesco Valenti, Nick Karcher, Lukas Grünhaupt, Ivan Takmakov, Patrick Winkel, Dennis Rieger, Alexey V. Ustinov, Nicolas Roch, Wolfgang Wernsdorfer, Kristel Michielsen, Oliver Sander, and Ioan M. Pop. Quantum nondemolition dispersive readout of a superconducting artificial atom using large photon numbers. Phys. Rev. Applied, 15:064030, Jun 2021. URL: https://link .aps .org/doi/10.1103/PhysRevApplied.15.064030, doi:10.1103/PhysRevApplied.15.064030.

$\left[\mathrm{HDSdG}^{+} 20\right]$ T. Hönigl-Decrinis, R. Shaikhaidarov, S.E. de Graaf, V.N. Antonov, and O.V. Astafiev. Twolevel system as a quantum sensor for absolute calibration of power. Phys. Rev. Applied, 13:024066, Feb 2020. URL: https: / / link.aps.org/doi/10.1103/PhysRevApplied. 13.024066, doi:10.1103/PhysRevApplied.13.024066. 
[HEDLZ12] Byeong Ho Eom, Peter K. Day, Henry G. LeDuc, and Jonas Zmuidzinas. A wideband, lownoise superconducting amplifier with high dynamic range. Nature Physics, 8(8):623-627, Aug 2012. doi:10.1038/nphys2356.

[HRS ${ }^{+}$17] A. Hosseinkhani, R.-P. Riwar, R. J. Schoelkopf, L. I. Glazman, and G. Catelani. Optimal configurations for normal-metal traps in transmon qubits. Phys. Rev. Applied, 8:064028, Dec 2017. URL: https://link.aps.org/doi/10.1103/PhysRevApplied.8.064028, doi:10.1103/PhysRevApplied.8.064028.

$\left[\mathrm{HVC}^{+} 19\right]$ Fabio Henriques, Francesco Valenti, Thibault Charpentier, Marc Lagoin, Clement Gouriou, Maria Martínez, Laura Cardani, Marco Vignati, Lukas Grünhaupt, Daria Gusenkova, and et al. Phonon traps reduce the quasiparticle density in superconducting circuits. Applied Physics Letters, 115(21):212601, Nov 2019. URL: http://dx.doi.org/10.1063/1. 5124967, doi:10.1063/1.5124967.

$\left[\mathrm{HWJ}^{+} 11\right]$ Io-Chun Hoi, C. M. Wilson, Göran Johansson, Tauno Palomaki, Borja Peropadre, and Per Delsing. Demonstration of a single-photon router in the microwave regime. Physical Review Letters, 107(7), Aug 2011. URL: http://dx.doi.org/10.1103/PhysRevLett.107. 073601, doi:10.1103/physrevlett.107.073601.

[IR18] Igor G. Irastorza and Javier Redondo. New experimental approaches in the search for axion-like particles. Progress in Particle and Nuclear Physics, 102:89159, 2018. URL: https://www.sciencedirect.com/science/article/pii/ S014664101830036X, doi:https://doi.org/10.1016/j.ppnp.2018.05.003.

$\left[\mathrm{JBM}^{+}\right.$14] P. Jung, S. Butz, M. Marthaler, M. V. Fistul, J. Leppäkangas, V. P. Koshelets, and A. V. Ustinov. Multistability and switching in a superconducting metamaterial. Nature Communications, 5(1), Apr 2014. URL: http://dx.doi.org/10.1038/ncomms4730, doi:10.1038/ncomms4730.

[JMS ${ }^{+}$12] J. E. Johnson, C. Macklin, D. H. Slichter, R. Vijay, E. B. Weingarten, John Clarke, and I. Siddiqi. Heralded state preparation in a superconducting qubit. Phys. Rev. Lett., 109:050506, Aug 2012. URL: https://link.aps.org/doi/10.1103/PhysRevLett.109.050506, doi:10.1103/PhysRevLett.109.050506.

[Jos62] B.D. Josephson. Possible new effects in superconductive tunnelling. Phys. Lett., 1(7):251253, 1962. doi:10.1016/0031-9163(62)91369-0.

[JSM ${ }^{+}$14] Evan Jeffrey, Daniel Sank, J. Y. Mutus, T. C. White, J. Kelly, R. Barends, Y. Chen, Z. Chen, B. Chiaro, A. Dunsworth, A. Megrant, P. J. J. O’Malley, C. Neill, P. Roushan, A. Vainsencher, J. Wenner, A. N. Cleland, and John M. Martinis. Fast accurate state measurement with superconducting qubits. Phys. Rev. Lett., 112:190504, May 2014. URL: https://link.aps.org/doi/10.1103/PhysRevLett.112.190504, doi: 10.1103/PhysRevLett.112.190504.

[Kan98] B. E. Kane. A silicon-based nuclear spin quantum computer. Nature, 393(6681):133-137, May 1998. doi:10.1038/30156.

$\left[\mathrm{KBF}^{+} 15\right]$ J. Kelly, R. Barends, A. G. Fowler, A. Megrant, E. Jeffrey, T. C. White, D. Sank, J. Y. Mutus, B. Campbell, Yu Chen, Z. Chen, B. Chiaro, A. Dunsworth, I. C. Hoi, C. Neill, P. J. J. O’Malley, C. Quintana, P. Roushan, A. Vainsencher, J. Wenner, A. N. Cleland, and John M. Martinis. State preservation by repetitive error detection in a superconducting quantum circuit. Nature, 
519(7541):66-69, Mar 2015. URL: http://dx.doi.org/10.1038/nature14270, doi: 10.1038/nature14270

$\left[\mathrm{KBS}^{+} 16\right]$ Philip Krantz, Andreas Bengtsson, Michä̈l Simoen, Simon Gustavsson, Vitaly Shumeiko, W. D. Oliver, C. M. Wilson, Per Delsing, and Jonas Bylander. Single-shot read-out of a superconducting qubit using a josephson parametric oscillator. Nature Communications, 7(1):11417, May 2016. doi:10.1038/ncomms11417.

[Kim08] H. J. Kimble. The quantum internet. Nature, 453(7198):1023-1030, Jun 2008. doi: 10.1038/nature07127.

$\left[\mathrm{KKY}^{+}\right.$19] P. Krantz, M. Kjaergaard, F. Yan, T. P. Orlando, S. Gustavsson, and W. D. Oliver. A quantum engineer's guide to superconducting qubits. Applied Physics Reviews, 6(2):021318, 2019. doi:10.1063/1.5089550.

[Kor16] Alexander N. Korotkov. Quantum bayesian approach to circuit qed measurement with moderate bandwidth. Phys. Rev. A, 94:042326, Oct 2016. URL: https : //link. aps . org/doi/ 10.1103/PhysRevA.94.042326, doi:10.1103/PhysRevA.94.042326.

$\left[\mathrm{KSB}^{+} 20\right]$ Morten Kjaergaard, Mollie E. Schwartz, Jochen Braumüller, Philip Krantz, Joel I.-J. Wang, Simon Gustavsson, and William D. Oliver. Superconducting qubits: Current state of play. Annual Review of Condensed Matter Physics, 11(1):369-395, Mar 2020. URL: http://dx . doi.org/10.1146/annurev-conmatphys-031119-050605, doi:10.1146/annurevconmatphys-031119-050605.

[KSK $\left.{ }^{+} 19\right]$ S. Krinner, S. Storz, P. Kurpiers, P. Magnard, J. Heinsoo, R. Keller, J. Lütolf, C. Eichler, and A. Wallraff. Engineering cryogenic setups for 100-qubit scale superconducting circuit systems. EPJ Quantum Technology, 6(1), May 2019. URL: http://dx.doi.org/10. 1140/epjqt/s40507-019-0072-0, doi:10.1140/epjqt/s40507-019-0072-0.

$\left[\mathrm{KSV}^{+} 18\right]$ A. Kou, W. C. Smith, U. Vool, I. M. Pop, K. M. Sliwa, M. Hatridge, L. Frunzio, and M. H. Devoret. Simultaneous monitoring of fluxonium qubits in a waveguide. Phys. Rev. Applied, 9:064022, Jun 2018. URL: https://link.aps.org/doi/10.1103/PhysRevApplied. 9.064022, doi:10.1103/PhysRevApplied.9.064022.

$\left[\mathrm{KTY}^{+} 21\right]$ Sunmi Kim, Hirotaka Terai, Taro Yamashita, Wei Qiu, Tomoko Fuse, Fumiki Yoshihara, Sahel Ashhab, Kunihiro Inomata, and Kouichi Semba. Enhanced coherence of all-nitride superconducting qubits epitaxially grown on silicon substrate. Communications Materials, 2(1):98, Sep 2021. doi:10.1038/s43246-021-00204-4.

$\left[\mathrm{KYG}^{+}\right.$07] Jens Koch, Terri M. Yu, Jay Gambetta, A. A. Houck, D. I. Schuster, J. Majer, Alexandre Blais, M. H. Devoret, S. M. Girvin, and R. J. Schoelkopf. Charge-insensitive qubit design derived from the cooper pair box. Physical Review A, 76(4), Oct 2007. URL: http://dx. doi.org/10.1103/PhysRevA.76.042319, doi:10.1103/physreva.76.042319.

$\left[\mathrm{LCC}^{+} 17\right]$ G. Liu, T.-C. Chien, X. Cao, O. Lanes, E. Alpern, D. Pekker, and M. Hatridge. Josephson parametric converter saturation and higher order effects. Applied Physics Letters, 111(20):202603, Nov 2017. URL: http://dx.doi.org/10.1063/1.5003032, doi: $10.1063 / 1.5003032$.

[LD98] Daniel Loss and David P. DiVincenzo. Quantum computation with quantum dots. Physical Review A, 57(1):120-126, Jan 1998. URL: http://dx.doi.org/10.1103/PhysRevA. 57.120, doi:10.1103/physreva.57.120. 
[Leh21] K. W. Lehnert. Quantum enhanced metrology in the search for fundamental physical phenomena. 2021. arXiv:2110.04912.

$\left[\mathrm{LNG}^{+} 18 \mathrm{a}\right]$ Yen-Hsiang Lin, Long B. Nguyen, Nicholas Grabon, Jonathan San Miguel, Natalia Pankratova, and Vladimir E. Manucharyan. Demonstration of protection of a superconducting qubit from energy decay. Phys. Rev. Lett., 120:150503, Apr 2018. URL: https://link.aps.org/doi/10.1103/PhysRevLett.120.150503, doi:10. 1103/PhysRevLett.120.150503.

$\left[\mathrm{LNG}^{+} 18 \mathrm{~b}\right]$ Yen-Hsiang Lin, Long B. Nguyen, Nicholas Grabon, Jonathan San Miguel, Natalia Pankratova, and Vladimir E. Manucharyan. Demonstration of protection of a superconducting qubit from energy decay. Phys. Rev. Lett., 120:150503, Apr 2018. URL: https://link.aps.org/doi/10.1103/PhysRevLett.120.150503, doi:10. 1103/PhysRevLett.120.150503.

$\left[\mathrm{LPP}^{+} 11\right]$ Florent Lecocq, Ioan M Pop, Zhihui Peng, Iulian Matei, Thierry Crozes, Thierry Fournier, Cécile Naud, Wiebke Guichard, and Olivier Buisson. Junction fabrication by shadow evaporation without a suspended bridge. Nanotechnology, 22(31):315302, jul 2011. doi:10.1088/0957-4484/22/31/315302.

$\left[\mathrm{MCD}^{+} 21\right]$ C. Monroe, W. C. Campbell, L.-M. Duan, Z.-X. Gong, A. V. Gorshkov, P. W. Hess, R. Islam, K. Kim, N. M. Linke, G. Pagano, P. Richerme, C. Senko, and N. Y. Yao. Programmable quantum simulations of spin systems with trapped ions. Rev. Mod. Phys., 93:025001, Apr 2021. URL: https://link.aps.org/doi/10.1103/RevModPhys.93.025001, doi: 10.1103/RevModPhys.93.025001.

[MCL19] Clemens Müller, Jared H Cole, and Jürgen Lisenfeld. Towards understanding two-levelsystems in amorphous solids: insights from quantum circuits. Reports on Progress in Physics, 82(12):124501, Oct 2019. URL: http://dx.doi.org/10.1088/1361-6633/ab3a7e, doi:10.1088/1361-6633/ab3a7e.

$\left[\mathrm{MGK}^{+} 18\right]$ N. Maleeva, L. Grünhaupt, T. Klein, F. Levy-Bertrand, O. Dupre, M. Calvo, F. Valenti, P. Winkel, F. Friedrich, W. Wernsdorfer, A. V. Ustinov, H. Rotzinger, A. Monfardini, M. V. Fistul, and I. M. Pop. Circuit quantum electrodynamics of granular aluminum resonators. Nature Communications, 9(1):3889, Sep 2018. doi:10.1038/s41467-018-06386-9.

[MKGD09] Vladimir E. Manucharyan, Jens Koch, Leonid I. Glazman, and Michel H. Devoret. Fluxonium: Single cooper-pair circuit free of charge offsets. Science, 326(5949):113-116, Oct 2009. URL: http://dx.doi.org/10.1126/science.1175552, doi:10.1126/ science.1175552.

[MM19] Alessandro Miano and Oleg A. Mukhanov. Symmetric traveling wave parametric amplifier. IEEE Transactions on Applied Superconductivity, 29(5):1-6, 2019. doi:10.1109/TASC . 2019. 2904699.

$\left[\mathrm{MMS}^{+}\right.$19] Z. K. Minev, S. O. Mundhada, S. Shankar, P. Reinhold, R. Gutiérrez-Jáuregui, R. J. Schoelkopf, M. Mirrahimi, H. J. Carmichael, and M. H. Devoret. To catch and reverse a quantum jump mid-flight. Nature, 570(7760):200-204, Jun 2019. URL: http: //dx.doi.org/10.1038/s41586-019-1287-z, doi:10.1038/s41586-019-1287-z.

$\left[\mathrm{MOH}^{+} 15\right]$ C Macklin, K O’Brien, D Hover, ME Schwartz, V Bolkhovsky, X Zhang, WD Oliver, and I Siddiqi. A near-quantum-limited josephson traveling-wave parametric amplifier. Science (New York, N.Y.), 350(6258):307-310, October 2015. doi:10.1126/science . aaa8525. 
[Mol69] B. R. Mollow. Power spectrum of light scattered by two-level systems. Phys. Rev., 188:19691975, Dec 1969. URL: https://link.aps.org/doi/10.1103/PhysRev.188.1969, doi:10.1103/PhysRev.188.1969.

$\left[\mathrm{MPB}^{+}\right.$19] M. Malnou, D. A. Palken, B. M. Brubaker, Leila R. Vale, Gene C. Hilton, and K. W. Lehnert. Squeezed vacuum used to accelerate the search for a weak classical signal. Phys. Rev. X, 9:021023, May 2019. URL: https://link.aps.org/doi/10.1103/PhysRevX. 9.021023, doi:10.1103/PhysRevX.9.021023.

[MPT20] Moein Malekakhlagh, Alexandru Petrescu, and Hakan E. Türeci. Lifetime renormalization of weakly anharmonic superconducting qubits. i. role of number nonconserving terms. Phys. Rev. B, 101:134509, Apr 2020. URL: https://link.aps.org/doi/10.1103/ PhysRevB.101.134509, doi:10.1103/PhysRevB.101.134509.

[MPW21] Eufemio Moreno-Pineda and Wolfgang Wernsdorfer. Measuring molecular magnets for quantum technologies. Nature Reviews Physics, 3(9):645-659, Sep 2021. doi:10.1038/ s42254-021-00340-3.

$\left[\mathrm{MVW}^{+} 21\right]$ M. Malnou, M.R. Vissers, J.D. Wheeler, J. Aumentado, J. Hubmayr, J.N. Ullom, and J. Gao. Three-wave mixing kinetic inductance traveling-wave amplifier with near-quantum-limited noise performance. PRX Quantum, 2:010302, Jan 2021. URL: https : / / ink.aps.org/ doi/10.1103/PRXQuantum.2.010302, doi:10.1103/PRXQuantum.2.010302.

$\left[\mathrm{MWB}^{+} 14\right]$ Josh Mutus, Ted White, Rami Barends, Yu Chen, Zijun Chen, Ben Chiaro, Andrew Dunsworth, Evan Jeffrey, Julian Kelly, Anthony Megrant, Charles Neill, Peter O’Malley, Pedram Roushan, Daniel Sank, Amit Vainsencher, James Wenner, Kyle Sundqvist, Andrew Cleland, and John Martinis. Strong environmental coupling in a josephson parametric amplifier. Applied Physics Letters, 104(26):263513, Jun 2014. URL: http: //dx.doi.org/10.1063/1.4886408, doi:10.1063/1.4886408.

$\left[\mathrm{MWG}^{+} 20\right]$ C. R. H. McRae, H. Wang, J. Gao, M. R. Vissers, T. Brecht, A. Dunsworth, D. P. Pappas, and J. Mutus. Materials loss measurements using superconducting microwave resonators. Review of Scientific Instruments, 91(9):091101, Sep 2020. URL: http://dx . doi .org/10.1063/ 5.0017378, doi:10.1063/5.0017378.

[MWHH21] Gary J. Mooney, Gregory A. L. White, Charles D. Hill, and Lloyd C. L. Hollenberg. Wholedevice entanglement in a 65-qubit superconducting quantum computer. Advanced Quantum Technologies, 4(10):2100061, Sep 2021. URL: http://dx.doi.org/10.1002/qute. 202100061 , doi:10.1002/qute.202100061.

[Neb20] Christoph E. Nebel. Chapter four - nitrogen-vacancy doped cvd diamond for quantum applications: A review. In Christoph E. Nebel, Igor Aharonovich, Norikazu Mizuochi, and Mutsuko Hatano, editors, Diamond for Quantum Applications Part 1, volume 103 of Semiconductors and Semimetals, pages 73-136. Elsevier, 2020. URL: https://www.sciencedirect.com/science/article/pii/ S0080878420300041, doi:https://doi.org/10.1016/bs.semsem.2020.03.004.

[NFE17] O. Naaman, D. G. Ferguson, and R. J. Epstein. High saturation power josephson parametric amplifier with ghz bandwidth. 2017. arXiv:1711.07549.

$\left[\mathrm{NLS}^{+}\right.$19] Long B. Nguyen, Yen-Hsiang Lin, Aaron Somoroff, Raymond Mencia, Nicholas Grabon, and Vladimir E. Manucharyan. High-coherence fluxonium qubit. Phys. Rev. X, 9:041041, 
Nov 2019. URL: https://link.aps.org/doi/10.1103/PhysRevX.9.041041, doi: 10.1103/PhysRevX.9.041041.

[NPT99] Y. Nakamura, Yu. A. Pashkin, and J. S. Tsai. Coherent control of macroscopic quantum states in a single-cooper-pair box. Nature, 398(6730):786-788, Apr 1999. doi:10.1038/19718.

$\left[\mathrm{NSH}^{+} 14\right]$ A. Narla, K. M. Sliwa, M. Hatridge, S. Shankar, L. Frunzio, R. J. Schoelkopf, and M. H. Devoret. Wireless josephson amplifier. Applied Physics Letters, 104(23):232605, 2014. doi:10.1063/1.4883373.

$\left[\mathrm{OAB}^{+} 08\right]$ Aaron D. O’Connell, M. Ansmann, R. C. Bialczak, M. Hofheinz, N. Katz, Erik Lucero, C. McKenney, M. Neeley, H. Wang, E. M. Weig, A. N. Cleland, and J. M. Martinis. Microwave dielectric loss at single photon energies and millikelvin temperatures. Applied Physics Letters, 92(11):112903, 2008. doi:10.1063/1.2898887.

[OMSZ] Kevin O'Brien, Chris Macklin, Irfan Siddiqi, and Xiang Zhang. doi:10.1103/ PhysRevLett.113.157001.

$\left[\mathrm{OPH}^{+} 16\right]$ Nissim Ofek, Andrei Petrenko, Reinier Heeres, Philip Reinhold, Zaki Leghtas, Brian Vlastakis, Yehan Liu, Luigi Frunzio, SM Girvin, L Jiang, Mazyar Mirrahimi, MH Devoret, and RJ Schoelkopf. Extending the lifetime of a quantum bit with error correction in superconducting circuits. Nature, 536(7617):441—445, August 2016. doi:10.1038/nature18949.

[OSB $\left.{ }^{+} 21\right]$ A. Osman, J. Simon, A. Bengtsson, S. Kosen, P. Krantz, D. P. Lozano, M. Scigliuzzo, P. Delsing, Jonas Bylander, and A. Fadavi Roudsari. Simplified josephson-junction fabrication process for reproducibly high-performance superconducting qubits. Applied Physics Letters, 118(6):064002, 2021. doi:10.1063/5.0037093.

$\left[\mathrm{OSF}^{+}\right.$19] Jonathan L. Ouellet, Chiara P. Salemi, Joshua W. Foster, Reyco Henning, Zachary Bogorad, Janet M. Conrad, Joseph A. Formaggio, Yonatan Kahn, Joe Minervini, Alexey Radovinsky, Nicholas L. Rodd, Benjamin R. Safdi, Jesse Thaler, Daniel Winklehner, and Lindley Winslow. First results from abracadabra- $10 \mathrm{~cm}$ : A search for sub- $\mu \mathrm{eV}$ axion dark matter. Phys. Rev. Lett., 122:121802, Mar 2019. URL: https://link.aps.org/doi/10.1103/ PhysRevLett.122.121802, doi:10.1103/PhysRevLett.122.121802.

[PDM ${ }^{+}$19] Luca Planat, Rémy Dassonneville, Javier Puertas Martínez, Farshad Foroughi, Olivier Buisson, Wiebke Hasch-Guichard, Cécile Naud, R. Vijay, Kater Murch, and Nicolas Roch. Understanding the saturation power of josephson parametric amplifiers made from squid arrays. Physical Review Applied, 11(3), Mar 2019. URL: http://dx.doi .org/10.1103/ PhysRevApplied.11.034014, doi:10.1103/physrevapplied.11.034014.

$\left[\mathrm{PFC}^{+}{ }^{12}\right]$ I. M. Pop, T. Fournier, T. Crozes, F. Lecocq, I. Matei, B. Pannetier, O. Buisson, and W. Guichard. Fabrication of stable and reproducible submicron tunnel junctions. Journal of Vacuum Science Technology B, Nanotechnology and Microelectronics: Materials, Processing, Measurement, and Phenomena, 30(1):010607, Jan 2012. URL: http: //dx.doi.org/10.1116/1.3673790, doi:10.1116/1.3673790.

[PGC $\left.{ }^{+} 14\right]$ Ioan M. Pop, Kurtis Geerlings, Gianluigi Catelani, Robert J. Schoelkopf, Leonid I. Glazman, and Michel H. Devoret. Coherent suppression of electromagnetic dissipation due to superconducting quasiparticles. Nature, 508(7496):369-372, Apr 2014. doi: 10.1038/nature13017. 
[PMT20] Alexandru Petrescu, Moein Malekakhlagh, and Hakan E. Türeci. Lifetime renormalization of driven weakly anharmonic superconducting qubits. ii. the readout problem. Phys. Rev. B, 101:134510, Apr 2020. URL: https://link.aps.org/doi/10.1103/PhysRevB.101. 134510, doi:10.1103/PhysRevB.101.134510.

$\left[\mathrm{PNW}^{+} 21\right]$ Kaidong Peng, Mahdi Naghiloo, Jennifer Wang, Gregory D Cunningham, Yufeng Ye, and Kevin P O'Brien. Near-ideal quantum efficiency with a floquet mode traveling wave parametric amplfier. 2021. arXiv:2104.08269.

[Poz11] David M Pozar. Microwave engineering; 4th ed. John Wiley Sons, New York, 2011.

$\left[\mathrm{PRD}^{+}\right.$20] Luca Planat, Arpit Ranadive, Rémy Dassonneville, Javier Puertas Martínez, Sébastien Léger, Cécile Naud, Olivier Buisson, Wiebke Hasch-Guichard, Denis M. Basko, and Nicolas Roch. Photonic-crystal josephson traveling-wave parametric amplifier. Phys. Rev. X, 10:021021, Apr 2020. URL: https://link.aps.org/doi/10.1103/PhysRevX.10.021021, doi: 10.1103/PhysRevX.10.021021.

$\left[\mathrm{PRM}^{+}\right.$21] Alexander P. M. Place, Lila V. H. Rodgers, Pranav Mundada, Basil M. Smitham, Mattias Fitzpatrick, Zhaoqi Leng, Anjali Premkumar, Jacob Bryon, Andrei Vrajitoarea, Sara Sussman, Guangming Cheng, Trisha Madhavan, Harshvardhan K. Babla, Xuan Hoang Le, Youqi Gang, Berthold Jäck, András Gyenis, Nan Yao, Robert J. Cava, Nathalie P. de Leon, and Andrew A. Houck. New material platform for superconducting transmon qubits with coherence times exceeding 0.3 milliseconds. Nature Communications, 12(1):1779, Mar 2021. doi:10. 1038/s41467-021-22030-5.

$\left[\mathrm{PSB}^{+} 15\right]$ S. Probst, F. B. Song, P. A. Bushev, A. V. Ustinov, and M. Weides. Efficient and robust analysis of complex scattering data under noise in microwave resonators. Review of Scientific Instruments, 86(2):024706, Feb 2015. URL: http: //dx.doi.org/10.1063/1.4907935, doi:10.1063/1.4907935.

[PTP46] E. M. Purcell, H. C. Torrey, and R. V. Pound. Resonance absorption by nuclear magnetic moments in a solid. Phys. Rev., 69:37-38, Jan 1946. URL: https ://link.aps.org/doi/ 10.1103/PhysRev.69.37, doi:10.1103/PhysRev.69.37.

$\left[\mathrm{RBT}^{+} 13\right]$ D. Ristè, C. C. Bultink, M. J. Tiggelman, R. N. Schouten, K. W. Lehnert, and L. DiCarlo. Millisecond charge-parity fluctuations and induced decoherence in a superconducting transmon qubit. Nature Communications, 4(1):1913, May 2013. doi : 10.1038/ncomms2936.

[RD16] Ananda Roy and Michel Devoret. Introduction to parametric amplification of quantum signals with josephson circuits. Comptes Rendus Physique, 17(7):740-755, Aug 2016. URL: http://dx.doi.org/10.1016/j.crhy.2016.07.012, doi:10.1016/j.crhy . 2016.07 .012$.

$\left[\mathrm{RDN}^{+} 12\right]$ M. D. Reed, L. DiCarlo, S. E. Nigg, L. Sun, L. Frunzio, S. M. Girvin, and R. J. Schoelkopf. Realization of three-qubit quantum error correction with superconducting circuits. Nature, 482(7385):382-385, Feb 2012. URL: http: / dx. doi .org/10 . 1038/nature10786, doi:10.1038/nature10786.

$\left[\mathrm{RDW}^{+} 12\right]$ D. Ristè, M. Dukalski, C. A. Watson, G. de Lange, M. J. Tiggelman, Ya. M. Blanter, K. W. Lehnert, R. N. Schouten, and L. DiCarlo. Deterministic entanglement of superconducting qubits by parity measurement and feedback. Nature, 502:350-354, Feb 2012. doi:10. 1038/nature12513. 
$\left[\mathrm{REP}^{+} 21\right]$ Arpit Ranadive, Martina Esposito, Luca Planat, Edgar Bonet, Cécile Naud, Olivier Buisson, Wiebke Guichard, and Nicolas Roch. A reversed kerr traveling wave parametric amplifier. 2021. arXiv:2101.05815.

$\left[\mathrm{RFN}^{+} 12\right]$ N. Roch, E. Flurin, F. Nguyen, P. Morfin, P. Campagne-Ibarcq, M. H. Devoret, and B. Huard. Widely tunable, nondegenerate three-wave mixing microwave device operating near the quantum limit. Phys. Rev. Lett., 108:147701, Apr 2012. URL: https://Iink.aps.org/ doi/10.1103/PhysRevLett.108.147701, doi:10.1103/PhysRevLett.108.147701.

[Rie22] D. Rieger. in prep. 2022.

$\left[\mathrm{RKC}^{+}\right]$Tanay Roy, Suman Kundu, Madhavi Chand, A. M. Vadiraj, A. Ranadive, N. Nehra, Meghan P. Patankar, J. Aumentado, A. A. Clerk, and R. Vijay. doi:10.1063/1.4939148.

$\left[\mathrm{RMA}^{+}{ }^{+}\right.$10] Sebastian Reick, Klaus Mølmer, Wolfgang Alt, Martin Eckstein, Tobias Kampschulte, Lingbo Kong, René Reimann, Alexander Thobe, Artur Widera, and Dieter Meschede. Analyzing quantum jumps of one and two atoms strongly coupled to an optical cavity. Journal of the Optical Society of America B, 27(6):A152, May 2010. URL: http://dx.doi.org/10. 1364/JOSAB.27.00A152, doi:10.1364/josab.27.00a152.

$\left[\right.$ RSP $^{+}$17] H. Rotzinger, S.T. Skacel, M Pfirrmann, J.N. Voss, J. Münzberg, S. Probst, P. Bushev, M.P. Weides, A.V. Ustinov, and J.E. Mooij. Aluminium-oxide wires for superconducting high kinetic inductance circuits. Superconductor science and technology, 30(2):025002, 2017. doi: 10 .1088/0953-2048/30/2/025002.

$\left[\mathrm{SBO}^{+} 11\right]$ Jeremy M. Sage, Vladimir Bolkhovsky, William D. Oliver, Benjamin Turek, and Paul B. Welander. Study of loss in superconducting coplanar waveguide resonators. Journal of Applied Physics, 109(6):063915, Mar 2011. URL: http://dx.doi.org/10.1063/1. 3552890, doi:10.1063/1.3552890.

$\left[\mathrm{SBW}^{+} 20\right]$ Alexander Stehli, Jan David Brehm, Tim Wolz, Paul Baity, Sergey Danilin, Valentino Seferai, Hannes Rotzinger, Alexey V. Ustinov, and Martin Weides. Coherent superconducting qubits from a subtractive junction fabrication process. Applied Physics Letters, 117(12):124005, 2020. doi:10.1063/5.0023533.

[Sch97] V.V. Schmidt. The Physics of Superconductors. Springer, Berlin, Heidelberg, 1997.

[SCID ${ }^{+}$16] P. Six, Ph. Campagne-Ibarcq, I. Dotsenko, A. Sarlette, B. Huard, and P. Rouchon. Quantum state tomography with noninstantaneous measurements, imperfections, and decoherence. Phys. Rev. A, 93:012109, Jan 2016. URL: https://link.aps .org/doi/10.1103/ PhysRevA.93.012109, doi:10.1103/PhysRevA.93.012109.

[SCK ${ }^{+}$16] Daniel Sank, Zijun Chen, Mostafa Khezri, J. Kelly, R. Barends, B. Campbell, Y. Chen, B. Chiaro, A. Dunsworth, A. Fowler, E. Jeffrey, E. Lucero, A. Megrant, J. Mutus, M. Neeley, C. Neill, P. J. J. O’Malley, C. Quintana, P. Roushan, A. Vainsencher, T. White, J. Wenner, Alexander N. Korotkov, and John M. Martinis. Measurement-induced state transitions in a superconducting qubit: Beyond the rotating wave approximation. Phys. Rev. Lett., 117:190503, Nov 2016. URL: https://link.aps.org/doi/10.1103/PhysRevLett.117.190503, doi:10.1103/PhysRevLett.117.190503.

[SCWS08] Davide Sarchi, Iacopo Carusotto, Michiel Wouters, and Vincenzo Savona. Coherent dynamics and parametric instabilities of microcavity polaritons in double-well systems. Phys. Rev. B, 
77:125324, Mar 2008. URL: https://link.aps.org/doi/10.1103/PhysRevB.77. 125324, doi:10.1103/PhysRevB.77.125324.

[SDH $\left.{ }^{+} 19\right]$ K. Serniak, S. Diamond, M. Hays, V. Fatemi, S. Shankar, L. Frunzio, R.J. Schoelkopf, and M.H. Devoret. Direct dispersive monitoring of charge parity in offset-charge-sensitive transmons. Phys. Rev. Applied, 12:014052, Jul 2019. URL: https : / / ink. aps .org/doi/ 10.1103/PhysRevApplied.12.014052, doi:10.1103/PhysRevApplied.12.014052.

[SDR $\left.{ }^{+} 12\right]$ L. Sun, L. DiCarlo, M. D. Reed, G. Catelani, Lev S. Bishop, D. I. Schuster, B. R. Johnson, Ge A. Yang, L. Frunzio, L. Glazman, M. H. Devoret, and R. J. Schoelkopf. Measurements of quasiparticle tunneling dynamics in a band-gap-engineered transmon qubit. Phys. Rev. Lett., 108:230509, Jun 2012. URL: https://link.aps.org/doi/10.1103/PhysRevLett. 108.230509, doi:10.1103/PhysRevLett.108.230509.

$\left[\mathrm{SFJ}^{+}\right.$19] V.V. Sivak, N.E. Frattini, V.R. Joshi, A. Lingenfelter, S. Shankar, and M.H. Devoret. Kerrfree three-wave mixing in superconducting quantum circuits. Physical Review Applied, 11(5), May 2019. URL: http://dx.doi.org/10.1103/PhysRevApplied.11.054060, doi:10.1103/physrevapplied.11.054060.

[SFM ${ }^{+}$21] Aaron Somoroff, Quentin Ficheux, Raymond A. Mencia, Haonan Xiong, Roman V. Kuzmin, and Vladimir E. Manucharyan. Millisecond coherence in a superconducting qubit. 2021. arXiv:2103.08578.

[Sio12] Neel highlights, table-top dilution cryostat: Sionludi, 2012. URL: http:/neel.cnrs . frimg/pdf/Table-top_dilution.pdf.

$\left[\mathrm{SKD}^{+}{ }^{16}\right]$ Muhandis Shiddiq, Dorsa Komijani, Yan Duan, Alejandro Gaita-Ariño, Eugenio Coronado, and Stephen Hill. Enhancing coherence in molecular spin qubits via atomic clock transitions. Nature, 531(7594):348-351, Mar 2016. doi:10.1038/nature16984.

$\left[\mathrm{SKV}^{+} 16\right]$ W. C. Smith, A. Kou, U. Vool, I. M. Pop, L. Frunzio, R. J. Schoelkopf, and M. H. Devoret. Quantization of inductively shunted superconducting circuits. Phys. Rev. B, 94:144507, Oct 2016. URL: https://link.aps.org/doi/10.1103/PhysRevB.94.144507, doi: 10.1103/PhysRevB.94.144507.

$\left[\right.$ SPL $^{+}$14] L. Sun, A. Petrenko, Z. Leghtas, B. Vlastakis, G. Kirchmair, K. M. Sliwa, A. Narla, M. Hatridge, S. Shankar, J. Blumoff, L. Frunzio, M. Mirrahimi, M. H. Devoret, and R. J. Schoelkopf. Tracking photon jumps with repeated quantum non-demolition parity measurements. $\mathrm{Na}$ ture, 511(7510):444-448, Jul 2014. URL: http://dx. doi .org/10.1038/nature13436, doi:10.1038/nature13436.

[SSL $\left.{ }^{+} 20\right]$ V. V. Sivak, S. Shankar, G. Liu, J. Aumentado, and M. H. Devoret. Josephson array-mode parametric amplifier. Physical Review Applied, 13(2), Feb 2020. URL: http://dx.doi.org/ 10.1103/PhysRevApplied.13.024014, doi:10.1103/physrevapplied.13.024014.

$\left[\mathrm{SVP}^{+}\right.$04] I. Siddiqi, R. Vijay, F. Pierre, C. M. Wilson, M. Metcalfe, C. Rigetti, L. Frunzio, and M. H. Devoret. Rf-driven josephson bifurcation amplifier for quantum measurement. Phys. Rev. Lett., 93:207002, Nov 2004. URL: https : / / ink.aps.org/doi/10.1103/PhysRevLett. 93. 207002, doi:10.1103/PhysRevLett.93.207002.

[SVW ${ }^{+}$20] Yannick Schön, Jan Nicolas Voss, Micha Wildermuth, Andre Schneider, Sebastian T. Skacel, Martin P. Weides, Jared H. Cole, Hannes Rotzinger, and Alexey V. Ustinov. Rabi oscillations 
in a superconducting nanowire circuit. npj Quantum Materials, 5(1):18, Mar 2020. doi: $10.1038 / s 41535-020-0220-x$.

[SWP $\left.{ }^{+} 19\right]$ Andre Schneider, Tim Wolz, Marco Pfirrmann, Martin Spiecker, Hannes Rotzinger, Alexey V. Ustinov, and Martin Weides. Transmon qubit in a magnetic field: Evolution of coherence and transition frequency. Phys. Rev. Research, 1:023003, Sep 2019. URL: https://link.aps.org/doi/10.1103/PhysRevResearch.1.023003, doi:10. 1103/PhysRevResearch.1.023003.

[Tin04] M Tinkham. Introduction to Superconductivity; 2nd ed. Dover Publications, Mineola, NY, 2004.

$\left[\mathrm{TKF}^{+} 19\right]$ S. Touzard, A. Kou, N. E. Frattini, V. V. Sivak, S. Puri, A. Grimm, L. Frunzio, S. Shankar, and M. H. Devoret. Gated conditional displacement readout of superconducting qubits. Phys. Rev. Lett., 122:080502, Feb 2019. URL: https://link.aps.org/doi/10.1103/ PhysRevLett.122.080502, doi:10.1103/PhysRevLett.122.080502.

$\left[\mathrm{TWF}^{+}\right.$21] I. Takmakov, P. Winkel, F. Foroughi, L. Planat, D. Gusenkova, M. Spiecker, D. Rieger, L. Grünhaupt, A.V. Ustinov, W. Wernsdorfer, I.M. Pop, and N. Roch. Minimizing the discrimination time for quantum states of an artificial atom. Phys. Rev. Applied, 15:064029, Jun 2021. URL: https://link. aps .org/doi/10.1103/PhysRevApplied.15.064029, doi:10.1103/PhysRevApplied.15.064029.

[VD17] Uri Vool and Michel Devoret. Introduction to quantum electromagnetic circuits. International Journal of Circuit Theory and Applications, 45(7):897-934, Jun 2017. URL: http: //dx. doi.org/10.1002/cta.2359, doi:10.1002/cta.2359.

[VDS09] R. Vijay, M. H. Devoret, and I. Siddiqi. Invited review article: The josephson bifurcation amplifier. Review of Scientific Instruments, 80(11):111101, 2009. doi : 10.1063/1. 3224703.

$\left[\mathrm{VHC}^{+}\right.$19] Francesco Valenti, Fabio Henriques, Gianluigi Catelani, Nataliya Maleeva, Lukas Grünhaupt, Uwe von Lüpke, Sebastian T. Skacel, Patrick Winkel, Alexander Bilmes, Alexey V. Ustinov, Johannes Goupy, Martino Calvo, Alain Benoît, Florence Levy-Bertrand, Alessandro Monfardini, and Ioan M. Pop. Interplay between kinetic inductance, nonlinearity, and quasiparticle dynamics in granular aluminum microwave kinetic inductance detectors. Phys. Rev. Applied, 11:054087, May 2019. URL: https://link.aps.org/doi/10.1103/ PhysRevApplied.11.054087, doi:10.1103/PhysRevApplied.11.054087.

[VPS ${ }^{+}$14] U. Vool, I. M. Pop, K. Sliwa, B. Abdo, C. Wang, T. Brecht, Y. Y. Gao, S. Shankar, M. Hatridge, G. Catelani, M. Mirrahimi, L. Frunzio, R. J. Schoelkopf, L. I. Glazman, and M. H. Devoret. Non-poissonian quantum jumps of a fluxonium qubit due to quasiparticle excitations. Phys. Rev. Lett., 113:247001, Dec 2014. URL: https://link.aps.org/doi/10. 1103/PhysRevLett.113.247001, doi:10.1103/PhysRevLett.113.247001.

[VSS11] R. Vijay, D. H. Slichter, and I. Siddiqi. Observation of quantum jumps in a superconducting artificial atom. Phys. Rev. Lett., 106:110502, Mar 2011. URL: https://Iink.aps.org/ doi/10.1103/PhysRevLett.106.110502, doi:10.1103/PhysRevLett.106.110502.

$\left[\mathrm{WBG}^{+} 20\right]$ Patrick Winkel, Kiril Borisov, Lukas Grünhaupt, Dennis Rieger, Martin Spiecker, Francesco Valenti, Alexey V. Ustinov, Wolfgang Wernsdorfer, and Ioan M. Pop. Implementation of a transmon qubit using superconducting granular aluminum. Physical Review X, 10(3), Aug 2020. URL: http: //dx . doi .org/10.1103/PhysRevX.10.031032, doi:10.1103/ physrevx.10.031032. 
[Win20] Oatrick Winkel. Superconducting quantumcircuits for hybrid architectures. PhD thesis, Karlsruhe Institute of Technology, 2020.

$\left[\mathrm{WKD}^{+}\right.$15] T. Weiß1, B. Küng, E. Dumur, A. K. Feofanov, I. Matei, C. Naud, O. Buisson, F. W. J. Hekking, and W. Guichard. Kerr coefficients of plasma resonances in josephson junction chains. Phys. Rev. B, 92:104508, Sep 2015. URL: https://link . aps .org/doi/10.1103/PhysRevB . 92.104508, doi:10.1103/PhysRevB.92.104508.

$\left[\mathrm{WKG}^{+} 17\right]$ T. Walter, P. Kurpiers, S. Gasparinetti, P. Magnard, A. Potočnik, Y. Salathé, M. Pechal, M. Mondal, M. Oppliger, C. Eichler, and A. Wallraff. Rapid high-fidelity single-shot dispersive readout of superconducting qubits. Phys. Rev. Applied, 7:054020, May 2017. URL: https://link.aps.org/doi/10.1103/PhysRevApplied.7.054020, doi:10. 1103/PhysRevApplied. 7.054020.

$\left[\mathrm{WMKS}^{+}\right.$16] Steven J. Weber, Kater W. Murch, Mollie E. Kimchi-Schwartz, Nicolas Roch, and Irfan Siddiqi. Quantum trajectories of superconducting qubits. Comptes Rendus Physique, 17(7):766-777, Aug 2016. URL: http: //dx . doi .org/10.1016/j .crhy.2016.07.007, doi:10.1016/j.crhy.2016.07.007.

[WQL14] Peiyue Wang, Lupei Qin, and Xin-Qi Li. Quantum bayesian rule for weak measurements of qubits in superconducting circuit QED. New Journal of Physics, 16(12):123047, Dec 2014. URL: http://dx.doi.org/10.1088/1367-2630/16/12/123047, doi: $10.1088 / 1367-2630 / 16 / 12 / 123047$.

$\left[\mathrm{WSB}^{+}\right.$04] A. Wallraff, D. I. Schuster, A. Blais, L. Frunzio, R. S. Huang, J. Majer, S. Kumar, S. M. Girvin, and R. J. Schoelkopf. Strong coupling of a single photon to a superconducting qubit using circuit quantum electrodynamics. Nature, 431(7005):162-167, Sep 2004. URL: http://dx.doi.org/10.1038/nature02851, doi:10.1038/nature02851.

$\left[\mathrm{WTR}^{+}\right.$20] Patrick Winkel, Ivan Takmakov, Dennis Rieger, Luca Planat, Wiebke Hasch-Guichard, Lukas Grünhaupt, Nataliya Maleeva, Farshad Foroughi, Fabio Henriques, Kiril Borisov, Julian Ferrero, Alexey V. Ustinov, Wolfgang Wernsdorfer, Nicolas Roch, and Ioan M. Pop. Nondegenerate parametric amplifiers based on dispersion-engineered josephson-junction arrays. Phys. Rev. Applied, 13:024015, Feb 2020. URL: https : //link.aps . org/doi/10.1103/ PhysRevApplied.13.024015, doi:10.1103/PhysRevApplied.13.024015.

$\left[\mathrm{XMZ}^{+} 20\right]$ Feihu Xu, Xiongfeng Ma, Qiang Zhang, Hoi-Kwong Lo, and Jian-Wei Pan. Secure quantum key distribution with realistic devices. Rev. Mod. Phys., 92:025002, May 2020. URL: https://link.aps.org/doi/10.1103/RevModPhys.92.025002, doi: 10.1103/RevModPhys.92.025002.

[YFA ${ }^{+}$17] Fumiki Yoshihara, Tomoko Fuse, Sahel Ashhab, Kosuke Kakuyanagi, Shiro Saito, and Kouichi Semba. Superconducting qubit-oscillator circuit beyond the ultrastrong-coupling regime. Nature Physics, 13(1):44-47, Jan 2017. doi:10.1038/nphys3906.

[YFMS] O. Yaakobi, L. Friedland, C. Macklin, and I. Siddiqi. doi : 10.1103/PhysRevB . 87.144301.

[YGK $\left.{ }^{+} 16\right]$ Fei Yan, Simon Gustavsson, Archana Kamal, Jeffrey Birenbaum, Adam P. Sears, David Hover, Ted J. Gudmundsen, Danna Rosenberg, Gabriel Samach, S. Weber, Jonilyn L. Yoder, Terry P. Orlando, John Clarke, Andrew J. Kerman, and William D. Oliver. The flux qubit revisited to enhance coherence and reproducibility. Nature Communications, 7(1):12964, Nov 2016. doi:10.1038/ncomms12964. 
[YGS ${ }^{+}$20] Fang Yang, Thomas Gozlinski, Tim Storbeck, Lukas Grünhaupt, Ioan M. Pop, and Wulf Wulfhekel. Microscopic charging and in-gap states in superconducting granular aluminum. Physical Review B, 102(10), Sep 2020. URL: http: //dx.doi .org/10.1103/PhysRevB . 102.104502, doi:10.1103/physrevb.102.104502.

$\left[Y^{+}{ }^{+}\right.$08] T. Yamamoto, K. Inomata, M. Watanabe, K. Matsuba, T. Miyazaki, W. D. Oliver, Y. Nakamura, and J. S. Tsai. Flux-driven josephson parametric amplifier. Applied Physics Letters, 93(4):042510, 2008. doi:10.1063/1.2964182.

$\left[\mathrm{YPN}^{+} 21\right]$ Yufeng Ye, Kaidong Peng, Mahdi Naghiloo, Gregory Cunningham, and Kevin P. O’Brien. Engineering purely nonlinear coupling between superconducting qubits using a quarton. Phys. Rev. Lett., 127:050502, Jul 2021. URL: https://link.aps.org/doi/10.1103/ PhysRevLett.127.050502, doi:10.1103/PhysRevLett.127.050502.

[YSK19] Xinyuan You, J. A. Sauls, and Jens Koch. Circuit quantization in the presence of timedependent external flux. Phys. Rev. B, 99:174512, May 2019. URL: https://Iink. aps. org/doi/10.1103/PhysRevB.99.174512, doi:10.1103/PhysRevB.99.174512.

$\left[Y_{S K}^{+}\right.$20] Fei Yan, Youngkyu Sung, Philip Krantz, Archana Kamal, David K. Kim, Jonilyn L. Yoder, Terry P. Orlando, Simon Gustavsson, and William D. Oliver. Engineering framework for optimizing superconducting qubit designs. 2020. arXiv:2006.04130.

[ZCCe21] Qingling Zhu, Sirui Cao, Fusheng Chen, and et.al. Quantum computational advantage via 60-qubit 24-cycle random circuit sampling. Science Bulletin, 2021. URL: https://www . sciencedirect.com/science/article/pii/S2095927321006733, doi:https:// doi.org/10.1016/j.scib.2021.10.017.

[Zor19] A.B. Zorin. Flux-driven josephson traveling-wave parametric amplifier. Phys. Rev. Applied, 12:044051, Oct 2019. URL: https://link. aps .org/doi/10.1103/PhysRevApplied. 12.044051, doi:10.1103/PhysRevApplied.12.044051. 



\section{List of Publications}

$\left[\mathrm{GKG}^{+} 20\right]$ Richard Gebauer, Nick Karcher, Daria Gusenkova, Martin Spiecker, Lukas Grünhaupt, Ivan Takmakov, Patrick Winkel, Luca Planat, Nicolas Roch, Wolfgang Wernsdorfer, Alexey V. Ustinov, Marc Weber, Martin Weides, Ioan M. Pop, and Oliver Sander. State preparation of a fluxonium qubit with feedback from a custom fpga-based platform. AIP Conference Proceedings, 2241(1):020015, 2020. doi:10.1063/5.0011721.

[GSG ${ }^{+}$19] Lukas Grünhaupt, Martin Spiecker, Daria Gusenkova, Nataliya Maleeva, Sebastian T. Skacel, Ivan Takmakov, Francesco Valenti, Patrick Winkel, Hannes Rotzinger, Alexey V. Ustinov, and Ioan M. Pop. Granular aluminium as a superconducting material for high-impedance quantum circuits. Nature Materials, 18(8):816-819, Apr 2019. URL: http://dx. doi .org/10 .1038/ s41563-019-0350-3, doi:10.1038/s41563-019-0350-3.

[GSG $\left.{ }^{+} 21\right]$ Daria Gusenkova, Martin Spiecker, Richard Gebauer, Madita Willsch, Dennis Willsch, Francesco Valenti, Nick Karcher, Lukas Grünhaupt, Ivan Takmakov, Patrick Winkel, Dennis Rieger, Alexey V. Ustinov, Nicolas Roch, Wolfgang Wernsdorfer, Kristel Michielsen, Oliver Sander, and Ioan M. Pop. Quantum nondemolition dispersive readout of a superconducting artificial atom using large photon numbers. Phys. Rev. Applied, 15:064030, Jun 2021. doi:10.1103/PhysRevApplied.15.064030.

$\left[\mathrm{TWF}^{+}\right.$21] I. Takmakov, P. Winkel, F. Foroughi, L. Planat, D. Gusenkova, M. Spiecker, D. Rieger, L. Grünhaupt, A.V. Ustinov, W. Wernsdorfer, I.M. Pop, and N. Roch. Minimizing the discrimination time for quantum states of an artificial atom. Phys. Rev. Applied, 15:064029, Jun 2021. doi:10.1103/PhysRevApplied.15.064029.

$\left[\mathrm{WTR}^{+}\right.$20] Patrick Winkel, Ivan Takmakov, Dennis Rieger, Luca Planat, Wiebke Hasch-Guichard, Lukas Grünhaupt, Nataliya Maleeva, Farshad Foroughi, Fabio Henriques, Kiril Borisov, Julian Ferrero, Alexey V. Ustinov, Wolfgang Wernsdorfer, Nicolas Roch, and Ioan M. Pop. Nondegenerate parametric amplifiers based on dispersion-engineered josephson-junction arrays. Phys. Rev. Applied, 13:024015, Feb 2020. doi:10.1103/PhysRevApplied.13.024015. 


\section{Acknowledgements}

First and foremost, I would like to thank my PhD advisor, Ioan Pop. You are an extraordinary scientist and teacher, and I consider myself lucky to have you as my supervisor. I learned an enormous amount from you, ranging from nanofabrication to giving a successful talk. Your leadership and enthusiasm create a warm and collegial atmosphere in the group, making work a pleasant experience. I will always be amazed by the fidelity of your scientific gut feelings.

I want to thank Wolfgang Wernsdorfer and Martina Esposito for the time and effort they are investing in my $\mathrm{PhD}$ defense. I am grateful to Wolfgang Wernsdorfer for the opportunity to use his facilities, and will always be inspired by his dedication and attitude to work.

I was lucky to have Nicolas Roch both as my Master project supervisor and collaborator. Thank you for the incredible discussions and the enormous amount of technical aspects of parametric amplifiers you showed to me. Your joy and enthusiasm are extremely contagious, and they will forever stay in my memory.

I thank all BFQ team members for the incredible four years. In particular, thanks to Dennis and Simon for the discussions, collaboration and for being the best duo I've seen. Thanks, Dasha, for the fun time we've spent in front of the fridge. Thanks, Martin, for the discussions. Thanks, Patrick, for the fruitful work we did together. Thanks, Francesco, for the HIQCing experience. Big thanks to the alumni: Lucas Grünhaupt, Natalia Maleeva, and Sebastian Skacel. I would also like to express my gratitude to the fluxon group members, especially to Jochen Braumüller.

I am grateful to Alexey Ustinov, Valeriy Ryazanov, and Oleg Astafiev for the opportunity to join their labs during my Bachelor and Master projects and for introducing me to the field. I would like to especially thank Alexey Ustinov for the summer internship in his group in KIT, which eventually made this PhD project possible.

I want to acknowledge the technical help of Lucas Radtke and Silvia Diewald, who made my cleanroom life as friendly as humanly possible. Thanks to Sascha Lukashenko for the help with infrared filters and for sharing his wisdom.

Я хочу поблагодарить своих родителей и Катю за их любовь и поддержку, без которых я не смог бы стать тем, кто я есть. 